\title{
Dose Errors in the Treatment Planning Process of Cancer Radiotherapy
}

\author{
by \\ W. Kenji Myint \\ M.Sc. (Carleton University) (2001)
}

\author{
A thesis submitted to the \\ Faculty of Graduate Studies and Research \\ in partial fulfillment of the requirements \\ for the degree of \\ Doctor of Philosophy
}

Ottawa-Carleton Institute for Physics

Department of Physics, Carleton University

Ottawa, Ontario, Canada

March, 2008

Copyright $\odot$ 2008, W. Kenji Myint 


$\begin{array}{ll}\begin{array}{l}\text { Library and } \\ \text { Archives Canada }\end{array} & \begin{array}{l}\text { Bibliothèque et } \\ \text { Archives Canada }\end{array} \\ \begin{array}{l}\text { Published Heritage } \\ \text { Branch }\end{array} & \begin{array}{l}\text { Direction du } \\ \text { Patrimoine de l'édition }\end{array} \\ \begin{array}{l}\text { 395 Wellington Street } \\ \text { Ottawa ON K1A 0N4 } \\ \text { Canada }\end{array} & \begin{array}{l}\text { 395, rue Wellington } \\ \text { Ottawa ON K1A 0N4 } \\ \text { Canada }\end{array}\end{array}$

Your file Votre référence ISBN: 978-0-494-40529-1

Ourfile Notre référence

ISBN: 978-0-494-40529-1

NOTICE:

The author has granted a nonexclusive license allowing Library and Archives Canada to reproduce, publish, archive, preserve, conserve, communicate to the public by telecommunication or on the Internet, loan, distribute and sell theses worldwide, for commercial or noncommercial purposes, in microform, paper, electronic and/or any other formats.

The author retains copyright ownership and moral rights in this thesis. Neither the thesis nor substantial extracts from it may be printed or otherwise reproduced without the author's permission.
AVIS:

L'auteur a accordé une licence non exclusive permettant à la Bibliothèque et Archives Canada de reproduire, publier, archiver, sauvegarder, conserver, transmettre au public par télécommunication ou par l'Internet, prêter, distribuer et vendre des thèses partout dans le monde, à des fins commerciales ou autres, sur support microforme, papier, électronique et/ou autres formats.

L'auteur conserve la propriété du droit d'auteur et des droits moraux qui protège cette thèse. $\mathrm{Ni}$ la thèse ni des extraits substantiels de celle-ci ne doivent être imprimés ou autrement reproduits sans son autorisation.
In compliance with the Canadian Privacy Act some supporting forms may have been removed from this thesis.

While these forms may be included in the document page count, their removal does not represent any loss of content from the thesis.
Conformément à la loi canadienne sur la protection de la vie privée, quelques formulaires secondaires ont été enlevés de cette thèse.

Bien que ces formulaires aient inclus dans la pagination, il n'y aura aucun contenu manquant.

\section{Canada}




\section{Abstract}

This thesis reports on the examination of specific dose errors in the treatment planning process. This process begins with the acquisition of the treatment planning CT (computed tomography) dataset and ends with the calculation of dose in the patient. The treatment planning $\mathrm{CT}$ is a Hounsfield unit (HU) representation of the patient that is converted to relative electron density in the treatment planning system. The treatment planning system utilizes a dose calculation algorithm to predict the dose based on the relative electron density distribution of the patient. The sources of dose error investigated in this thesis can be categorized as: i) errors in the HU representation of the patient; ii) errors in the relative electron density distribution of the patient; and iii) errors in the dose calculation algorithm.

Errors in the dose calculation algorithms were examined in Chapter 3, where the accuracy of the Theraplan Plus treatment planning system's implementation of the pencil beam and collapsed cone convolution algorithms were investigated in lung-equivalent material. Both algorithms had difficulty modeling the broadening of the beam in the lung-equivalent material but the collapsed cone convolution algorithm generally showed consistently smaller dose errors than the pencil beam algorithm. As expected, the pencil beam model could not model any lateral electron transport and the largest dose errors were observed near lateral lung-acrylic interfaces. In chapter 4, objects present during dose delivery but not accounted for in the treatment planning CT dataset were 
investigated. These can be categorized as errors in the HU representation of the patient. One such example is the treatment tabletop present during delivery, but replaced with a different table during the CT scan. In this study, the attenuation of the beam by a carbon fiber treatment tabletop was quantified and a practical solution to account for the tabletop was proposed. It was determined that neglecting the attenuation of oblique treatment fields by the carbon fiber couch can result in localized dose reduction from $4 \%$ to $16 \%$ depending on energy, field size, and geometry. To remedy this attenuation, the results showed that incorporating the carbon fiber couch in the TPS patient model reduces the dose error to less than 2\% (using Theraplan Plus v3.8). In chapter 5, dose errors in the presence of high- $Z$ prosthetic devices were reported, representing errors in both the HU and electron density distribution of the patient. This study also investigated the reduction in dose error from using treatment planning calculations based on MVCT images rather than conventional kVCT images. The MVCT-based calculations showed consistently smaller dose errors than kVCT-based calculations for both pencil beam and superposition algorithms. The reconstruction artifacts were greatly reduced with the use of MVCT, giving a much more accurate HU representation of the patient. MVCT images also improved the reliability of the electron density map. 


\section{Acknowledgements}

I would like to thank my thesis supervisor, Dr. Lee H. Gerig for his knowledge and guidance throughout my Ph.D. studies. Lee provided a great learning environment at the Ottawa Hospital Cancer Centre (OHCC), encouraging me to expand my knowledge of radiotherapy as much as possible. I would also like to thank senior physicists Dr. Miller MacPherson and Dr. David Wilkins at the OHCC. Miller was an invaluable resource for many aspects of my research, especially for my work on Monte Carlo simulations. David provided a steady guiding force throughout my years at the OHCC, and I'm grateful for all of his insightful discussions. I am grateful to Prof. Paul Johns and Prof. Bog Jarosz from the Carleton University Physics department for their on-going encouragement and guidance, and for giving me this opportunity. I would also like to thank all of my fellow graduate students at the OHCC physics lab for our daily banter and goodwill.

I am indebted to my parents for their endless encouragement and support. I've gained so much strength from their unwavering belief in anything I do.

Finally, I am forever grateful to my wife, Christine. My Ph.D. would not have been possible without her enduring love, patience, understanding and encouragement. 


\section{Table of Contents}

$\begin{array}{lr}\text { Abstract } & \text { ii } \\ \text { Acknowledgements } & \text { iv } \\ \text { List of Figures } & \text { viii } \\ \text { List of Tables } & \text { xvi } \\ \text { List of Abbreviations } & \text { xvii }\end{array}$

1 Introduction 1

1.1 Radiation therapy 2

1.1.1 Stages of radiation therapy 2

1.1.1.1 Treatment planning process 5

$\begin{array}{lll}1.2 & \text { Dose delivery accuracy } & 6\end{array}$

$\begin{array}{lll}1.3 & \text { Guide to the thesis } & 7\end{array}$

2 Monte Carlo modeling of a linear accelerator treatment head 10

$\begin{array}{lll}2.1 \text { Introduction } & 10\end{array}$ 
2.2 Components of a linear accelerator beam head

$\begin{array}{lll}2.3 & \text { EGSnrc simulation parameters } & 18\end{array}$

$\begin{array}{ll}2.4 \text { Composition of materials } & 19\end{array}$

2.5 Optimizing beam model parameters 23

2.5.1 Monitor unit conversion 32

3 Treatment planning dose calculations in low $\mathrm{Z}$ materials 35

3.1 Introduction 35

3.2 Dose calculation algorithms 38

3.2.1 Convolution / superposition models $\quad 39$

3.2.1.1 Collapsed cone convolution 43

3.2.1.2 Multi-grid superposition $\quad 44$

$\begin{array}{lll}\text { 3.2.1.3 Pencil beam algorithm } & 45\end{array}$

$\begin{array}{lll}3.3 & \text { Lung dose measurements and calculations } & 47\end{array}$

$\begin{array}{lll}\text { 3.3.1 Materials and methods } & 47\end{array}$

3.3.1.1 Measurement and Calculation Error $\quad 54$

3.3.1.2 Thermo-luminescent Dosimeters (TLDs) 55

3.3.2 Treatment planning system lung dose errors $\quad 59$

$\begin{array}{lll}\text { 3.3.3 Discussion } & 88\end{array}$

$\begin{array}{lll}3.3 .4 & \text { Conclusions } & 97\end{array}$

4 Treatment dose errors due to beam attenuation by a carbon fiber tabletop 99

$\begin{array}{lll}4.1 & \text { Abstract } & 99\end{array}$

$\begin{array}{lll}4.2 & \text { Introduction } & 100\end{array}$

$\begin{array}{lll}\text { 4.3 Materials and methods } & 102\end{array}$

$\begin{array}{lll}4.4 & \text { Results } & 107\end{array}$

$\begin{array}{lll}4.5 & \text { Discussion } & 110\end{array}$

$\begin{array}{lll}4.6 & \text { Conclusions } & 114\end{array}$ 
5 Treatment planning dose errors in the presence of hip and spinal prostheses 115

$\begin{array}{ll}5.1 \text { Introduction } & 115\end{array}$

5.1.1 MVCT image quality 118

$\begin{array}{ll}\text { 5.1.2 Aim of study } & 120\end{array}$

$\begin{array}{ll}5.2 \text { Materials and methods } & 120\end{array}$

$\begin{array}{ll}\text { 5.2.1 Treatment planning dose calculations } & 120\end{array}$

5.2.2 Monte Carlo simulation parameters 134

5.2.3 Monte Carlo simulation phantom 135

$\begin{array}{lll}5.3 \text { Results } & 141\end{array}$

$\begin{array}{lll}5.4 \text { Discussion } & 167\end{array}$

6 Conclusions 181

$\begin{array}{lll}6.1 & \text { Future work } & 184\end{array}$

A Thermo-luminescent dosimeter (TLD) reproducibility statistics $\quad 187$

$\begin{array}{ll}\text { References } & 189\end{array}$ 


\section{List of Figures}

1.1 A summary of the stages in the radiation therapy process.

2.1 Schematic drawings of the Siemens Mevatron beam head components modeled in BEAMnrc for a) 6-MV and b) 18-MV photon beams.

2.2 A schematic diagram of the secondary collimators and reticle components of the Siemens Mevatron beam head.

2.3 Incident electron energy spectra examined for the optimization of the Primus linear accelerator treatment head for an $18 \mathrm{MV}$ beam modeled by BEAMnrc.

2.4 Central axis depth-dose curves in a homogeneous water phantom for a 6MV, $10 \times 10 \mathrm{~cm}^{2}$ field measured with diodes and modeled using the EGSnrc / BEAMnrc / DOSXYZnrc system.

2.5 Beam profiles at depths of $1.6,10$, and $20 \mathrm{~cm}$ in a homogeneous water phantom for a $6 \mathrm{MV}, 10 \times 10 \mathrm{~cm}^{2}$ field measured with diodes and modeled using the EGSnrc / BEAMnrc / DOSXYZnrc system.

2.6 Central axis depth-dose curves in a homogeneous water phantom for an 18 $\mathrm{MV}, 10 \times 10 \mathrm{~cm}^{2}$ field measured with diodes and modeled using the EGSnrc / BEAMnrc / DOSXYZnre system.

2.7 Beam profiles at depths of $1.6,10$, and $20 \mathrm{~cm}$ in a homogeneous water phantom for an $18 \mathrm{MV}, 10 \times 10 \mathrm{~cm}^{2}$ field measured with diodes and modeled using the EGSnrc / BEAMnrc / DOSXYZnrc system.

3.1 Diagram of the acrylic phantom containing two lung equivalent inhomogeneities.

3.2 Beams eye view of a plane through the middle of the phantom $(\mathrm{z}=0 \mathrm{~cm}$, see Figure 3.2). 
3.3 Measured (TLD) and calculated (PB and CCC) dose profiles in lung equivalent material for a $6 \mathrm{MV}$ photon beam in the $5 \times 5 i$ beam geometry at four depths below the proximal lung-acrylic interface (depths $=0.0 \mathrm{~cm}$, $1.0 \mathrm{~cm}, 3.9 \mathrm{~cm}$ and $7.9 \mathrm{~cm}$ ).

3.4 The \% dose difference between the calculated (PB and CCC) and TLD measurements for a $6 \mathrm{MV}$ photon beam in the $5 \times 5 i$ experimental geometry at four depths below the proximal lung-acrylic interface.

3.5 Measured (TLD) and calculated (PB and CCC) dose profiles in lung equivalent material for a $10 \mathrm{MV}$ photon beam in the $5 \times 5 i$ beam geometry at four depths below the proximal lung-acrylic interface (depths $=0.0 \mathrm{~cm}$, $1.0 \mathrm{~cm}, 3.9 \mathrm{~cm}$ and $7.9 \mathrm{~cm}$ ).

3.6 The \% dose difference between the calculated (PB and CCC) and TLD measurements for a $10 \mathrm{MV}$ photon beam in the $5 \times 5 i$ experimental geometry at four depths below the proximal lung-acrylic interface.

3.7 Measured (TLD) and calculated (PB and CCC) dose profiles in lung equivalent material for a $18 \mathrm{MV}$ photon beam in the $5 \times 5 i$ beam geometry at four depths below the proximal lung-acrylic interface (depths $=0.0 \mathrm{~cm}$, $1.0 \mathrm{~cm}, 3.9 \mathrm{~cm}$ and $7.9 \mathrm{~cm}$ ).

3.8 The \% dose difference between the calculated (PB and CCC) and TLD measurements for a $18 \mathrm{MV}$ photon beam in the $5 \times 5 i$ experimental geometry at four depths below the proximal lung-acrylic interface..

3.9 Measured (TLD) and calculated (PB and CCC) dose profiles in lung equivalent material for a $6 \mathrm{MV}$ photon beam in the $5 \times 5 i$ i beam geometry at four depths below the proximal lung-acrylic interface (depths $=0.0 \mathrm{~cm}$, $1.0 \mathrm{~cm}, 3.9 \mathrm{~cm}$ and $7.9 \mathrm{~cm}$ ).

3.10 The \% dose difference between the calculated (PB and CCC) and TLD measurements for a $6 \mathrm{MV}$ photon beam in the $5 \times 5 i i$ experimental geometry at four depths below the proximal lung-acrylic interface.

3.11 Measured (TLD) and calculated (PB and CCC) dose profiles in lung equivalent material for a $10 \mathrm{MV}$ photon beam in the $5 \times 5 i i$ beam geometry at four depths below the proximal lung-acrylic interface (depths $=0.0 \mathrm{~cm}$, $1.0 \mathrm{~cm}, 3.9 \mathrm{~cm}$ and $7.9 \mathrm{~cm}$ ). 
3.12 The \% dose difference between the calculated (PB and CCC) and TLD measurements for a $10 \mathrm{MV}$ photon beam in the $5 \times 5 i i$ experimental geometry at four depths below the proximal lung-acrylic interface.

3.13 Measured (TLD) and calculated (PB and CCC) dose profiles in lung equivalent material for a $18 \mathrm{MV}$ photon beam in the $5 \times 5 i i$ beam geometry at four depths below the proximal lung-acrylic interface (depths $=0.0 \mathrm{~cm}$, $1.0 \mathrm{~cm}, 3.9 \mathrm{~cm}$ and $7.9 \mathrm{~cm}$ ).

3.14 The \% dose difference between the calculated (PB and CCC) and TLD measurements for a $18 \mathrm{MV}$ photon beam in the $5 \times 5 i i$ experimental geometry at four depths below the proximal lung-acrylic interface.

3.15 Measured (TLD) and calculated (PB and CCC) dose profiles in lung equivalent material for a $6 \mathrm{MV}$ photon beam in the $10 \times 10$ beam geometry at four depths below the proximal lung-acrylic interface (depths $=0.0 \mathrm{~cm}$, $1.0 \mathrm{~cm}, 3.9 \mathrm{~cm}$ and $7.9 \mathrm{~cm}$ ).

3.16 The \% dose difference between the calculated (PB and CCC) and TLD measurements for a $6 \mathrm{MV}$ photon beam in the $10 \times 10$ experimental geometry at four depths below the proximal lung-acrylic interface.

3.17 Measured (TLD) and calculated (PB and CCC) dose profiles in lung equivalent material for a $10 \mathrm{MV}$ photon beam in the $10 \times 10$ beam geometry at four depths below the proximal lung-acrylic interface (depths $=0.0 \mathrm{~cm}, 1.0 \mathrm{~cm}, 3.9 \mathrm{~cm}$ and $7.9 \mathrm{~cm}$ ).

3.18 The \% dose difference between the calculated (PB and CCC) and TLD measurements for a $10 \mathrm{MV}$ photon beam in the $10 \times 10$ experimental geometry at four depths below the proximal lung-acrylic interface.

3.19 Measured (TLD) and calculated (PB and CCC) dose profiles in lung equivalent material for a $18 \mathrm{MV}$ photon beam in the $10 \times 10$ beam geometry at four depths below the proximal lung-acrylic interface (depths $=0.0 \mathrm{~cm}, 1.0 \mathrm{~cm}, 3.9 \mathrm{~cm}$ and $7.9 \mathrm{~cm}$ ).

3.20 The \% dose difference between the calculated (PB and CCC) and TLD measurements for a $18 \mathrm{MV}$ photon beam in the $10 \times 10$ experimental geometry at four depths below the proximal lung-acrylic interface. 
3.21 The penumbra width as a function of lung depth for the $5 \times 5 i$ geometry for a) $6 \mathrm{MV}$ b) $10 \mathrm{MV}$ and c) $18 \mathrm{MV}$ photon beams.

3.22 The penumbra width as a function of nominal beam energy fro the $5 \times 5 i$ geometry at a depth midway $($ depth $=3.9 \mathrm{~cm})$ through the lung volume.

3.23 Relative dose difference between treatment planning calculations (pencil beam and collapsed-cone convolution algorithms) and TLD measurements as a function of nominal photon beam energy with the $5 \times 5 i$ geometry at four depths below the proximal lung-acrylic interface.

3.24 Relative dose difference between treatment planning calculations (pencil beam and collapsed-cone convolution algorithms) and TLD measurements as a function of nominal photon beam energy with the $5 \times 5 i i$ geometry at four depths below the proximal lung-acrylic interface.

3.25 Relative dose difference between treatment planning calculations (pencil beam and collapsed-cone convolution algorithms) and TLD measurements as a function of nominal photon beam energy with the $10 \times 10$ geometry at four depths below the proximal lung-acrylic interface.

4.1 The experimental geometry for in-phantom measurement of dose reduction, calculated by the ratio of readings (a) with and (b) without the couch.

4.2 Longitudinal intersection of the beam with the treatment couch.

4.3 Theraplan isodose distributions calculated without (top) and with (bottom) the treatment couch included.

4.4 The dose reduction from attenuation by the tabletop at various positions along its length.

5.1 Images of the prosthetic devices used in this study are shown in a) hip prosthesis \#1 consisting of a femoral stem and head, b) hip prosthesis \#2 consisting only of the femoral stem and c) the spinal rod prosthesis.

5.2 Diagrams illustrating cross-sectional slices of the a) hip prostheses phantom and b) spinal rod phantom. Ion chamber measurements were taken at a depth of $6.8 \mathrm{~cm}$ and the hole was plugged during CT imaging. 
5.3 KVCT (a) and MVCT (b) images of the wax phantom containing hip prostheses showing the differences in the artefacts in the two image types.

5.4 KVCT (a) and MVCT (b) images of the wax phantom containing the spinal prosthesis showing the differences in the artefacts in the two image types.

5.5 Cross-sectional MVCT slices illustrating the beam geometries examined with the a) hip prostheses and b) spinal prosthesis phantoms.

5.6 Relative electron density curves for both the kVCT and MVCT imagers, comparing Hounsfield units and MVCT numbers over the KVCT range.

5.7 The mass density $\left(\rho_{\mathrm{m}}\right)$ and the electron density relative to water $\left(\rho_{\mathrm{e}}\right)$ plotted as a function of the MVCT Hounsfield units.

5.8 MVCT HU profiles through titanium alloy hip prostheses in a solid wax phantom before and after streak artifacts were removed from the wax region.

5.9 Cross-sectional images of the hip prostheses phantom a) before and b) after streak artifacts were removed from the wax region.

5.10 MVCT number profiles through titanium alloy spinal prosthesis and Styrofoam blocks in a solid wax phantom before and after streak artifacts were removed from the wax region.

5.11 Cross-sectional images of the spinal prosthesis phantom a) before and b) after streak artifacts were removed from the wax region.

5.12 Comparing treatment planning system (Theraplan Plus v3.8 and CMS) and Monte Carlo based EGSnrc dose calculations in a $30 \times 30 \times 30 \mathrm{~cm}^{3}$ water phantom for an $18 \mathrm{MV}, 10 \times 10 \mathrm{~cm}^{2}$ field at $100 \mathrm{~cm}$ SAD delivering $100 \mathrm{MUs}$.

5.13 Comparing treatment planning system (Theraplan Plus v3.8 and CMS) and Monte Carlo based EGSnrc dose calculations in a $30 \times 30 \times 30 \mathrm{~cm}^{3}$ water phantom for an $18 \mathrm{MV}, 10 \times 10 \mathrm{~cm}^{2}$ field at $100 \mathrm{~cm}$ SAD delivering 100 MUs. 
5.14 kVCT and MVCT based dose profiles calculated with Monte Carlo, pencil beam and superposition algorithms for a $5 \times 5 \mathrm{~cm}^{2} 6 \mathrm{MV}$ photon beam incident on the central axis (beam setup \#1 in Figure 5.5) of a hip prostheses phantom.

5.15 kVCT and MVCT based dose profiles calculated with Monte Carlo, pencil beam and superposition algorithms for a $10 \times 10 \mathrm{~cm}^{2} 6 \mathrm{MV}$ photon beam incident on the central axis (beam setup \#1 in Figure 5.5) of a hip prostheses phantom.

5.16 The dose difference between treatment planning calculations (pencil beam and superposition algorithms) and MC simulations for a $5 \times 5 \mathrm{~cm}^{2} 6 \mathrm{MV}$ photon beam incident on the central axis (beam setup \#1 in Figure 5.5) of a hip prostheses phantom. The depth of the profiles is shown in Figure 5.8 .

5.17 The dose difference between treatment planning calculations (pencil beam and superposition algorithms) and MC simulations for a $10 \times 10 \mathrm{~cm}^{2} 6$ MV photon beam incident on the central axis (beam setup \#1 in Figure 5.5) of a hip prostheses phantom. The depth of the profiles is shown in Figure 5.8.

5.18 kVCT and MVCT based dose profiles calculated with Monte Carlo, pencil beam and superposition algorithms for a $5 \times 5 \mathrm{~cm}^{2} 18 \mathrm{MV}$ photon beam incident on the central axis (beam setup \#1 in Figure 5.5) of a hip prostheses phantom.

5.19 kVCT and MVCT based dose profiles calculated with Monte Carlo, pencil beam and superposition algorithms for a $10 \times 10 \mathrm{~cm}^{2} 18 \mathrm{MV}$ photon beam incident on the central axis (beam setup \#1 in Figure 5.5) of a hip prostheses phantom.

5.20 The dose difference between treatment planning calculations (pencil beam and superposition algorithms) and MC simulations for a $5 \times 5 \mathrm{~cm}^{2} 18 \mathrm{MV}$ photon beam incident on the central axis (beam setup \#1 in Figure 5.5) of a hip prostheses phantom. The depth of the profiles is shown in Figure 5.8 .

5.21 The dose difference between treatment planning calculations (pencil beam and superposition algorithms) and MC simulations for a $10 \times 10 \mathrm{~cm}^{2} 18$ MV photon beam incident on the central axis (beam setup \#1 in Figure 
5.5) of a hip prostheses phantom. The depth of the profiles is shown in Figure 5.8.

5.22 kVCT and MVCT based dose profiles calculated with Monte Carlo, pencil beam and superposition algorithms for a $10 \times 10 \mathrm{~cm}^{2} 6 \mathrm{MV}$ photon beam incident on the hip prosthesis at the central axis depth (beam setup \#2 in Figure 5.5) of a wax phantom. The depth of the profiles is shown in Figure 5.8

5.23 kVCT and MVCT based depth-dose profiles calculated with Monte Carlo, pencil beam and superposition algorithms for a $10 \times 10 \mathrm{~cm}^{2} 6 \mathrm{MV}$ photon beam incident on the hip prosthesis at the central axis depth (beam setup \#2 in Figure 5.5) of a wax phantom. The depth of the profiles is shown in Figure 5.8.

5.24 The dose difference between treatment planning calculations (pencil beam and superposition algorithms) and MC simulations for a $10 \times 10 \mathrm{~cm}^{2} 6$ MV photon beam incident on the hip prosthesis at the central axis depth (beam setup \#2 in Figure 5.5) of a wax phantom. The depth of the profiles is shown in Figure 5.8.

5.25 kVCT and MVCT based dose profiles calculated with Monte Carlo, pencil beam and superposition algorithms for a $5 \times 5 \mathrm{~cm}^{2} 6 \mathrm{MV}$ photon beam incident on the central axis (beam setup \#3 in Figure 5.5) of a spinal prosthesis phantom.

5.26 kVCT and MVCT based dose profiles calculated with Monte Carlo, pencil beam and superposition algorithms for a $10 \times 10 \mathrm{~cm}^{2} 6 \mathrm{MV}$ photon beam incident on the central axis (beam setup \#3 in Figure 5.5) of a spinal prosthesis phantom.

5.27 The dose difference between treatment planning calculations (pencil beam and superposition algorithms) and MC simulations for a $5 \times 5 \mathrm{~cm}^{2} 6 \mathrm{MV}$ photon beam incident on the central axis (beam setup \#3 in Figure 5.5) of a spinal prosthesis phantom.

5.28 The dose difference between treatment planning calculations (pencil beam and superposition algorithms) and MC simulations for a $10 \times 10 \mathrm{~cm}^{2} 6$ MV photon beam incident on the central axis (beam setup \#3 in Figure 5.5) of a spinal prosthesis phantom. 
5.29 kVCT and MVCT based dose profiles calculated with Monte Carlo, pencil beam and superposition algorithms for a $5 \times 5 \mathrm{~cm}^{2} 18 \mathrm{MV}$ photon beam incident on the central axis (beam setup \#3 in Figure 5.5) of a spinal prosthesis phantom.

5.30 kVCT and MVCT based dose profiles calculated with Monte Carlo, pencil beam and superposition algorithms for a $10 \times 10 \mathrm{~cm}^{2} 18 \mathrm{MV}$ photon beam incident on the central axis (beam setup \#3 in Figure 5.5) of a spinal prosthesis phantom.

5.31 The dose difference between treatment planning calculations (pencil beam and superposition algorithms) and MC simulations for a $5 \times 5 \mathrm{~cm}^{2} 18 \mathrm{MV}$ photon beam incident on the central axis (beam setup \#3 in Figure 5.5) of a spinal prosthesis phantom.

5.32 The dose difference between treatment planning calculations (pencil beam and superposition algorithms) and MC simulations for a $10 \times 10 \mathrm{~cm}^{2} 18$ MV photon beam incident on the central axis (beam setup \#3 in Figure 5.5) of a spinal prosthesis phantom.

5.33 Conventional CT images of a patient (a) with and (b) without high-Z fillings present in the oral cavity.

5.34 CT images of a patient with high-Z hip prostheses taken with (a) kilovoltage and (b) megavoltage X-rays.

5.35 kVCT (Hounsfield unit) and MVCT number profiles from images of titanium alloy hip prostheses in a solid wax phantom.

5.36 Electron density profiles (relative to water) from kVCT and MVCT images (unedited) of titanium alloy hip prostheses in a solid wax phantom. 


\section{List of Tables}

2.1 BEAMnrc defined (default) materials with cross-sectional data calculated by PEGS4, based on density effect corrections in ICRU Report 37.

2.2 Additional mixtures, compounds and elements specific to the Siemens Mevatron linear accelerator.

2.3 The dose calibration factors used to convert BEAMnrc / DOSXYZnrc dose outputs (cGy per incident electron) to units of $\mathrm{cGy} / \mathrm{MU}$.

3.1 Relative electron density (RED) and Hounsfield unit (HU) data for the Philips AcQsim large bore single slice CT simulator (Philips Medical Systems, ON, Canada).

4.1 In-air and in-phantom measurements of the effect of beam attenuation by the Medtec clinical treatment tabletop.

4.2 The difference between measured and calculated (TPP - Theraplan Plus) dose for 6-MV and 18-MV fields with and without attenuation from the treatment tabletop.

5.1 Elemental composition of hip and spinal prosthetic devices used in this study.

5.2 A comparison of the mass density, electron density relative to water (RED) and MVCT Hounsfield unit (HU) data of various compounds.

A.1 Accuracy and reproducibility of TLD chips (Box 3 and 4) irradiated with 100 cGy. The data was derived from three consecutive calibration experiments. 


\section{List of Abbreviations}

\begin{tabular}{|c|c|}
\hline $\mathrm{CBCT}$ & Cone-beam computed tomography \\
\hline $\mathrm{CCC}$ & Collapsed-cone convolution \\
\hline CT & Computed tomography \\
\hline DCF & Dose calibration factor \\
\hline DVH & Dose-volume histogram \\
\hline EBRT & External beam radiation therapy \\
\hline EDK & Energy deposition kernel \\
\hline EGS & Electron gamma shower \\
\hline FOV & Field-of-view \\
\hline FWHM & Full-width half-maximum \\
\hline $\mathrm{HU}$ & Hounsfield unit \\
\hline ICRU & International Commission on Radiation Units and Measurements \\
\hline ISL & Inverse square law \\
\hline $\mathrm{kVCT}$ & Kilovoltage computed tomography \\
\hline LINAC & Linear accelerator \\
\hline $\mathrm{MC}$ & Monte Carlo \\
\hline MGS & Multi-grid superposition \\
\hline MLC & Multi-leaf collimator \\
\hline MRI & Magnetic resonance imaging \\
\hline MU & Monitor unit \\
\hline MVCT & Megavoltage computed tomography \\
\hline NSCLC & Non-small cell lung cancer \\
\hline NTCP & Normal tissue complication probability \\
\hline $\mathrm{OHCC}$ & Ottawa Hospital Cancer Centre \\
\hline PET & Positron emission tomography \\
\hline RED & Relative electron density \\
\hline RT & Radiation therapy \\
\hline SAD & Source-to-axis distance \\
\hline SNR & Signal-to-noise ratio \\
\hline TCP & Tumour-control probability \\
\hline TERMA & Total energy released per unit mass \\
\hline TLD & Thermo-luminescent dosimeter \\
\hline TPR & Tissue-phantom ratio \\
\hline TPS & Treatment planning system \\
\hline
\end{tabular}




\section{Chapter 1}

\section{Introduction}

Cancer is a disease affecting more and more Canadians with each passing year. Based on current incidence rates in Canada, $38 \%$ of women and $44 \%$ of men will develop cancer during their lifetimes (Canadian Cancer Statistics 2006). The mortality rates show that approximately 1 in 4 Canadians will die from cancer. Cancer represents a class of diseases that are characterized by an uncontrolled division of cells and an ability of these cells to spread throughout the human body. Cancerous cells are able to spread directly into adjacent tissues or through the bloodstream and lymphatic system to metastasize to distant sites. There are many different types of cancer and many different forms of treatment. The most common forms of treatment are surgery, chemotherapy, radiation therapy, high-intensity focused ultrasound and hormone therapy, used individually or in conjunction with one another. It is estimated that nearly one half of new cancer patients will be treated with radiation therapy (Canadian Cancer Statistics 2006). 


\subsection{Radiation Therapy}

Radiation therapy is a specialized technique for the treatment of cancer with the use of ionizing radiation. The ideal goal of treatment is to deliver a precise radiation dose to the tumour volume while minimizing dose and hence damage to surrounding healthy tissue. Radiation can be delivered externally via external beam radiation therapy (EBRT) or internally via brachytherapy. EBRT is the application of radiation that has been produced by an external source such as a linear accelerator or a $\mathrm{Co}^{60}$ unit. Conversely, brachytherapy involves the insertion of a radioactive source internally and in close proximity to the treatment area. The work in this thesis deals exclusively with external beam photon radiation therapy applications.

\subsubsection{Stages of radiation therapy}

In practice, there are a number of stages involved in the process, all of which are necessary components of a successful treatment. A flow chart illustrating the general stages is shown in Figure 1.1 (IAEA 2003, ICRU 50 1993, ICRU 62 1999, Perez et al 1997). The process begins with the clinical evaluation of the patient through a series of diagnostic tests (step \#1). These tests include CT and MR scans and a histological analysis of a sample of the cancerous tissue, obtained via biopsy or surgery. Based on these tests, physicians form a decision regarding the therapeutic direction of the treatment (step \#2). 


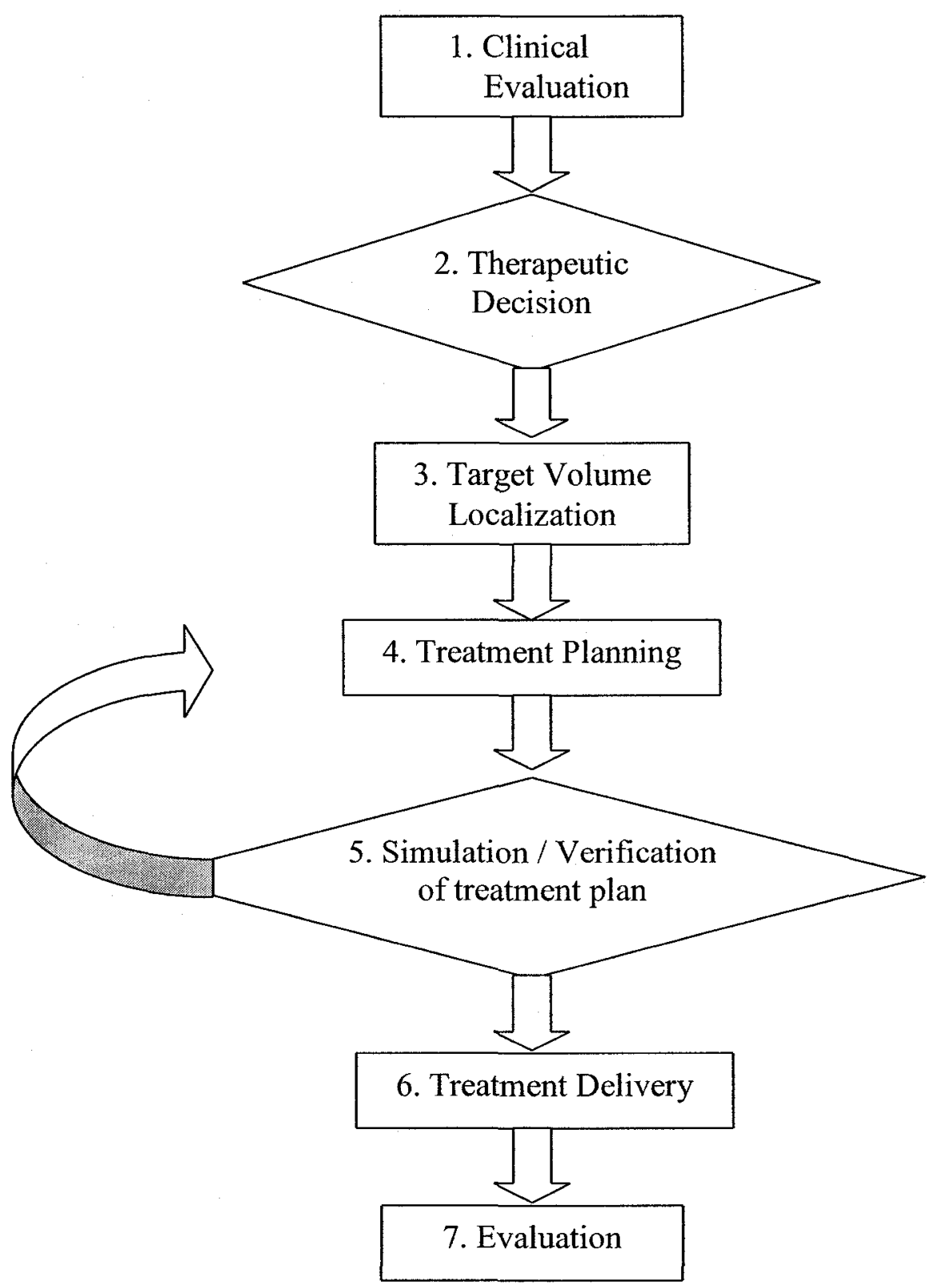

Figure 1.1 A summary of the stages in the radiation therapy process. 
If radiation therapy is chosen as part of the overall treatment, the radiation oncologist will localize the target (tumour) volume as well as any sensitive critical organs (step \#3). A dosimetrist may play a supportive role in this stage as well. Treatment planning is a broad term incorporating multiple stages and members of staff in the process (step \#4). The patient is scanned in the CT (computed tomography) simulator to form a CT dataset used specifically for treatment planning dose calculations. Radiation oncologists analyze plans and select an initial treatment plan for the patient. The dosimetrists utilize the treatment planning software to set up the appropriate beams, shielding components, and other treatment aids to perform dose calculations based on the treatment planning CT dataset. Physicists ensure that the planning system has accurate beam data, geometry, and dose computation and support the radiation oncologists and dosimetrists.

The next stage is the simulation and verification of the treatment plan (step \#5). The radiation oncologist will examine the results of the initial treatment planning dose calculation and determine whether it satisfies the objectives of the treatment. If it is deemed unsatisfactory, the treatment plan is adjusted accordingly and the dose calculations are re-simulated and re-verified. This cycle between treatment planning, simulation, and verification is re-iterated until the radiation oncologist is satisfied with the plan. Once the plan has been finalized, the patient can begin the radiation treatment stage, usually broken into multiple daily fractions over lengthy periods of time (step \#6). Many staff members contribute to this stage of the process including physicists, and radiation therapists and oncologists. The last stage is the final evaluation of the patient 
by the radiation oncologist to determine whether or not the treatment was successful (step \#7).

\subsubsection{Treatment planning process}

The treatment planning stage begins with the acquisition of the treatment planning CT dataset and ends with the calculation of dose in the patient. The treatment planning CT dataset represents a virtual patient divided into a series of slices. Each slice contains a matrix of pixels (or voxels since each slice has finite thickness), each representing a Hounsfield Unit (HU) that is based on the mean attenuation of the tissue within that voxel. The Hounsfield Unit is defined as:

$$
H U=\frac{\mu_{x}-\mu_{\text {water }}}{\mu_{\text {water }}} \times 1000
$$

where $\mu_{\mathrm{x}}$ and $\mu_{\mathrm{water}}$ are the linear attenuation coefficients of a material and water, respectively. The $\mathrm{HU}$ for air is -1000 , water is 0 , and compounds such as normal tissue and bone can range depending on the type. Many clinical CT simulators are designed not to exceed a maximum Hounsfield unit of 3000 . Thus, any highly attenuating materials will saturate at this level. In the treatment planning system (TPS), the HU is converted to relative electron density based on calibration measurements performed by the physicist. Once the beam geometry and relevant treatment parameters have been specified, the TPS utilizes the relative electron density distribution of the patient to determine the predicted dose. 


\subsection{Dose delivery accuracy}

Each step in the radiotherapy process contributes to the overall uncertainty in the treatment. The dose delivery process begins after a therapeutic decision is made to treat the patient with radiation therapy and ends with the delivery of radiation to the patient (steps (3) to (7) in Figure 1.1). It has been shown that small changes in dose can result in large changes in the local response of the tissue and in the tumour control probability (Mijnheer et al 1987, Sanchez-Nieto and Nahum 1999). In 1988, Boyer and Schultheiss suggested that a $1 \%$ higher accuracy in delivered dose results in a $2 \%$ increase in cure. Hence, extensive research has been done to identify and improve the numerous sources of dose error in the dose delivery process.

In 1976, the International Commission on Radiation Units and Measurements (ICRU) formed guidelines to improve the accuracy and quality of reporting dose. ICRU Reports 50 and 62 recommend that the delivered dose be within $+7 \%$ and $-5 \%$ of the prescribed dose. In 1987, Mijnheer et al formulated criteria for accuracy in the delivery of dose to a patient during photon or neutron therapy. Their recommendations were based on the relative steepness of dose-effect curves for local tumour control and normal tissue complication. The sources of uncertainty were categorized into random and systematic, with a recommendation that the total uncertainty in the dose delivery be within 3.5\%. Although a single value was recommended, it was recognized that a larger value would be acceptable in many cases and a smaller value would be required for special cases. In 1988, Brahme came to similar conclusions while investigating the dose 
accuracy requirements of external beam therapy with electrons and photons ${ }^{[9]}$. His recommendations were that the dose be delivered within $\pm 3 \%$.

Several studies have quantified the uncertainties in each step of the dose delivery process, where the accuracy of the dose delivery is determined by the sum of all uncertainties (Dutreix 1984, Essers and Mijnheer 1999, Goitein 1985, Mijnheer et al 1987). This calculation is becoming increasingly more complex with advances in treatment equipment and techniques. Hence, it is difficult to achieve dose accuracy within $3 \%$ and most clinics aim for the more conservative ICRU 50 recommendation of $5 \%$. These recommendations form a guideline for the dose delivery errors investigated in this thesis.

\subsection{Guide to the Thesis}

The aim of this work was to investigate specific dose errors in the treatment planning stage of the dose delivery process. The treatment planning steps can be simplified by the following flow diagram:

$$
H U \stackrel{\text { Transform }}{\longrightarrow} \rho_{e} \stackrel{T P S}{\longrightarrow} D_{\text {calc }}
$$

where HU represents the Hounsfield Unit, $\rho_{\mathrm{e}}$ is the electron density and $\mathrm{D}_{\text {calc }}$ is the dose calculated by the TPS. This thesis focuses on sources of dose error within this chain of events and proposes practical solutions to minimize these errors in the clinic.

Chapter 2 describes the modeling of a clinical linear accelerator treatment head with the EGSnrc / BEAMnrc system. This was developed as a tool for experiments 
performed in Chapter 5 and so the methodology is described in this chapter. The EGSnrc system performs a Monte Carlo based simulation of photon and electron transport in arbitrary geometries. BEAMnrc was designed to simulate the radiation beams from photon and electron radiotherapy sources.

Chapter 3 describes treatment planning dose calculations in low atomic number materials, specifically lung-equivalent material. The focus will be on quantifying the lung dose accuracy of two separate algorithms (pencil beam and collapsed cone convolution) implemented in the Theraplan Plus version 3.8 with Oncentra DCM version 2.0 treatment planning system. Limitations in the treatment planning calculations are addressed as well as errors in the inherent assumptions of the dose calculation algorithms. These highlight errors in $D_{\text {calc }}$ of the treatment planning process as it pertains to lowdensity materials.

Chapter 4 examines dose errors resulting from elements that are present during treatment but not present in the treatment planning CT dataset. These signify errors in the HU representation of the patient in the treatment planning process. Attenuation of the treatment beam by a carbon fiber treatment tabletop is a prime example of this inconsistency and the potential dose error is quantified in this chapter. A practical clinical solution to minimize the dose error is subsequently discussed.

Chapter 5 discusses treatment planning dose errors in the presence of high atomic number materials. This introduces multiple sources of error affecting the accuracy of 
both the $\mathrm{HU}$ and $\rho_{\mathrm{e}}$ representations of the patient. These errors were investigated by performing kilovoltage CT-based treatment planning dose calculations on phantoms containing titanium hip and spinal prostheses and comparing these results with MC dose calculations. Furthermore, megavoltage CT-based treatment planning was examined to improve the accuracy of predicted dose calculations for patients with high- $\mathrm{Z}$ prostheses. 


\section{Chapter 2}

\section{Monte Carlo modeling of a linear accelerator treatment head}

\subsection{Introduction}

This chapter reviews the modeling of a clinical linear accelerator treatment head at the Ottawa Hospital Cancer Centre (OHCC). This model was developed as a tool for the research reported in Chapter 5. A separate chapter is devoted to this methodology due to the extensive time and nature of developing the tool.

Monte Carlo (MC) methods use random numbers and probability statistics to model complex physical systems. The general principle is to find a numerical solution by randomly sampling from a mathematical or physical relationship. Though statistical sampling has been practiced since the early 1900 s, it was only after the electronic computer was developed (1945) that MC methods were studied in depth. In the 1970s, 
$\mathrm{MC}$ techniques were introduced into medical physics applications, but were limited to simple geometries because of a lack of both computing power and developed MC codes.

By the late 1970s, MC techniques were already being used as a computational method of determining photon spectra from various sources (McCall et al 1978). In the mid 1980s, simple electron beams and targets were being modeled for MC simulations and producing energy spectrums in air and in phantoms. These results were then used to calculate useful dosimetric quantities such as stopping power ratios (Andreo 1988, Andreo and Nahum 1985, Andreo and Brahme 1986,). At around the same time, Rogers et al $(1985,1990)$ were already using MC simulations to determine ion chamber responses, as well as calculate wall attenuation and scatter corrections for ion chambers. Models for linear accelerators slowly progressed between 1970 and 1990 but this pace increased dramatically by the mid 1990s. This can be credited to the development of several new MC codes along with increasingly more powerful and economical computing resources. In 1995, Rogers et al introduced the BEAM/EGS4 user interface for the modeling of medical radiation therapy linear accelerators. In 2002, this was upgraded to the BEAMnrc/EGSnrc system and has since become one of the most widely used radiotherapy MC codes (Rogers et al 2002). It has been benchmarked against measurements for photon and electron beams from linear accelerators and $\mathrm{Co}^{60}$ units (Deng et al 2004, Faddegon et al 2005, Heath and Seuntjens 2003, Pena et al 2004).

The strength of $\mathrm{MC}$ techniques for radiotherapy dose calculations is that they begin with first principles and track individual particle histories. Therefore, they take 
into account the transport of secondary particles. Conventional treatment planning algorithms are limited by approximations made to model electron transport as well as approximations made in the characterization of the beam. In contrast to conventional treatment planning algorithms, $\mathrm{MC}$ techniques can produce accurate results in regions of tissue inhomogeneities and surface irregularities.

The main disadvantage of MC methods is the computational time required to get sufficient statistical accuracy in the dose calculations. To overcome this drawback, researchers developed faster MC-based algorithms with sufficient accuracy and efficiency for radiotherapy clinics while at the same time being aided by the technological advancements in computer processing speed (Fippel 1999, Kawrakow 2000, Keall and Hoban 1996, Sempau et al 2000). In addition, generalized models of the radiation source of the treatment unit were being developed so that users could tune the models to match their individual clinical treatment units (Fippel et al 2003, Fix et al 2004, Jiang et al 2001).

This chapter will summarize the steps involved in modeling and validating a clinical linear accelerator beam head. The treatment unit (Siemens Mevatron, Serial \#3742, Siemens Canada Ltd.) modeled in this section was also used for all physical measurements and treatment planning calculations. This permitted the direct comparison of both in-phantom dose measurements and planning calculations with $\mathrm{MC}$ simulations. 


\subsection{Components of a linear accelerator beam head}

The EGSnrc / BEAMnrc system was used to model the clinical linear accelerator. The EGS (Electron Gamma Shower) system performs a Monte Carlo simulation of electron and photon transport in arbitrary geometries. It is specialized to simulate particles with energies ranging between a few $\mathrm{keV}$ to several hundreds of $\mathrm{GeV}$ (Kawrakow and Rogers 2006). BEAMnrc was designed to simulate the radiation beams from any radiotherapy source including electron and photon beams from linear accelerators, low-energy $\mathrm{x}$-rays and $\mathrm{Co}^{60}$ units. It is used in conjunction with the EGSnrc code system (Rogers et al 1995).

One of the strengths of the BEAM system is that it can produce a phase-space output of a beam at any specified plane in the model. This phase-space file contains information on the position, energy, direction, charge and history tag for each particle, and can be re-used for simulation within BEAMnrc, as an input for dose calculation in a patient or phantom, or stored as an efficient means of characterizing a specific beam. Our treatment head model was segmented into two main sections. The first section modeled all components up to the collimators and the second modeled all components of the collimators such as the jaws and multi-leaf collimator (MLC) (as will be discussed in the following paragraphs). The advantage of this was that it allowed for one phase-space file to be created per photon beam energy in the first section and then a phase-space file for each field size in the second section. 
Another strength of the BEAMnrc system is that it offers a simple means of modeling a linear accelerator beam head. The model is composed of a series of component modules, each representing a specific geometric shape or combination of geometric shapes. The user specifies which components are required for their individual treatment unit and assigns the exact dimensions and position of each component. Figure 2.1 (a) and (b) illustrate the components specified for the first section of our 6- and 18MV Siemens Mevatron beam model, respectively. Figure 2.2 shows the position of the components for a $10 \times 10 \mathrm{~cm}^{2}$ field at 100 source-to-axis distance (SAD). These components comprise the second section of our beam model. The jaws in the middle portion of the diagram represent an incomplete visualization of the MLC system. The MLC leaves are parallel to the y-axis and are not visible in the xz-plane.

The simulation begins with a primary electron beam (with a specified energy spectrum, trajectory, and spatial distribution) hitting a high- $Z$ target resulting in the production of bremsstrahlung photons. The photons are collimated with the primary collimator and form a distribution in which there is a higher intensity along the beam central axis and progressively less intensity away from it. The peaked distribution is made uniform by a conical metal absorber called a flattening filter, which absorbs more photons from the central axis and fewer from the periphery of the beam. In some accelerators, there exists a wedge-shaped compensator at the base of the flattening filter to account for the non-uniform initial electron beam incident on the target. This component was not necessary in our model since the electron beam was given a Gaussian spatial distribution, which is axially symmetrical. The next component is the monitor 
chamber that usually consists of a dual ionization chamber system that samples the radiation beam passing through the treatment head and produces electrical signals that terminate the treatment when the prescribed dose is given. The two ionization chambers are independent, with one serving as a check on the other. The final component of the first section is the mirror that reflects a field-defining light that illuminates the area to be irradiated on the patient's skin. It duplicates the size and shape of the x-ray field incident on the patient as defined by the collimators or other beam-limiting devices.

The second section of the model focuses on components that shape the photon beam. The treatment field size is defined by four thick tungsten blocks or two blocks combined with an MLC system. The design of the blocks allows the face of the jaws to adjust to the diverging beam angle as it moves outward or inward. The edge of each jaw is rounded and moves laterally as opposed to an arc motion. The MLC enables the formation of conformal fields by dividing a pair of jaws into multiple segmented leaves, each of which can be individually positioned for projection to $1 \mathrm{~cm}$ width at isocenter. The movement of the MLC leaves is in the shape of an arc so that the face of the leaves provide a flat edge to the diverging beam and hence a sharply defined field. The final component of the beam model is a thin polycarbonate exit window called the reticle. The final phase-space output of the beam model was measured $50 \mathrm{~cm}$ below the top of the target, with all areas not specified by any components filled with air. 
a)

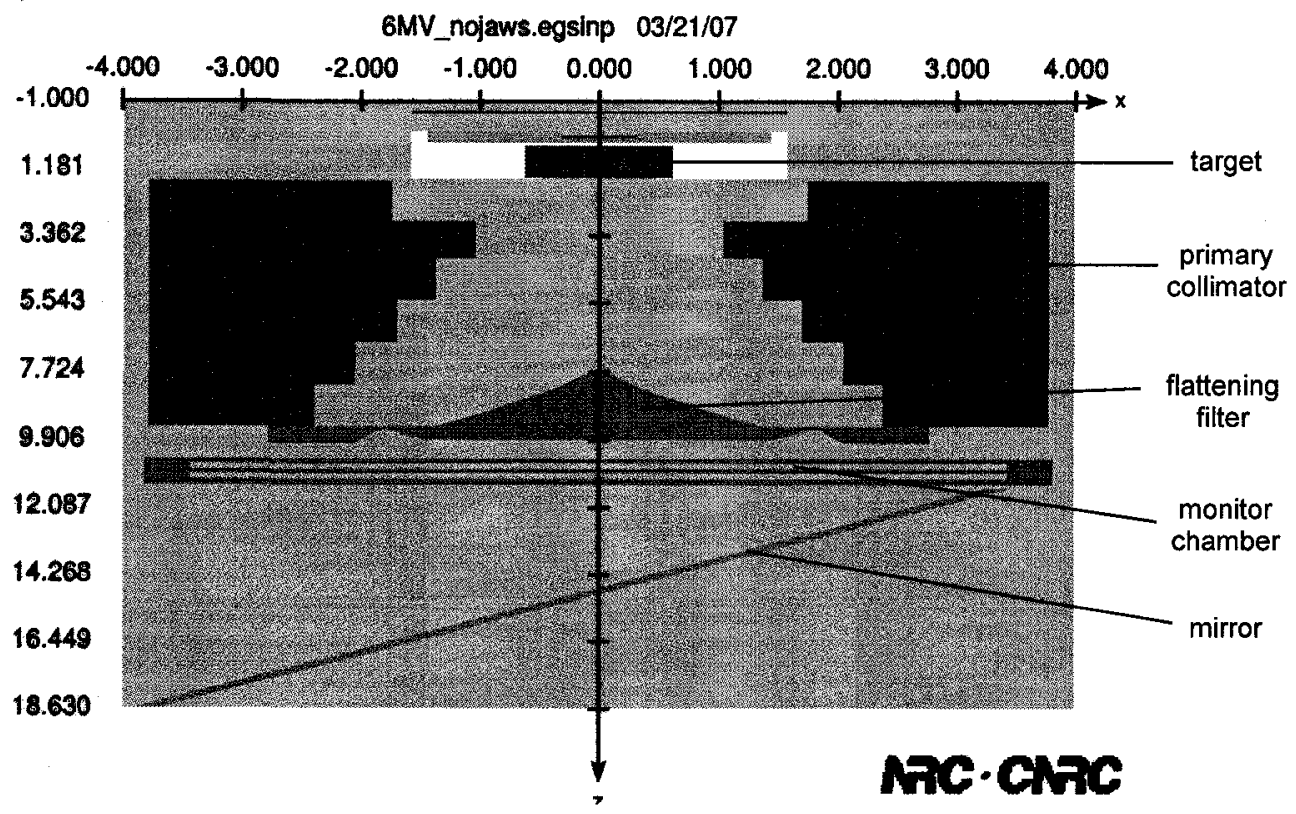

b)

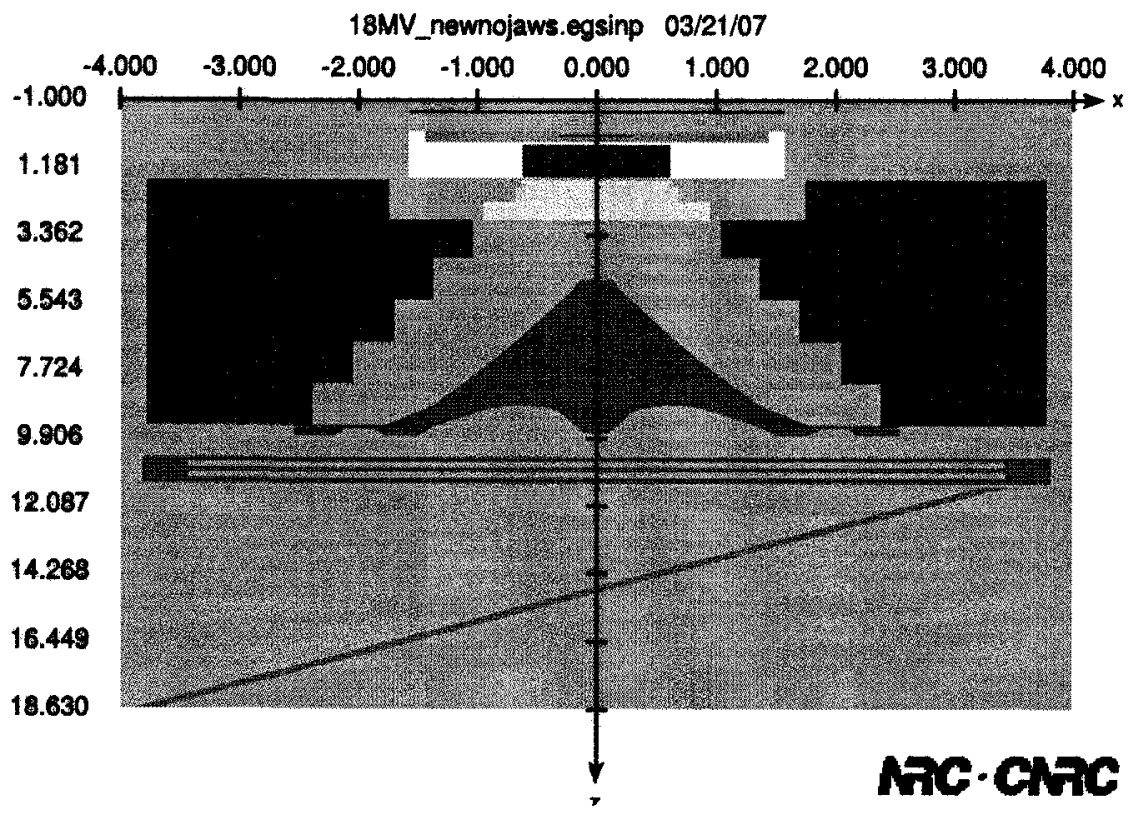

Figure 2.1 Schematic drawings of the Siemens Mevatron beam head components modeled in BEAMnrc for a) 6-MV and b) 18-MV photon beams. These components comprise the first section of our beam model. (Diagrams provided by BEAMnrc accelerator preview function) 


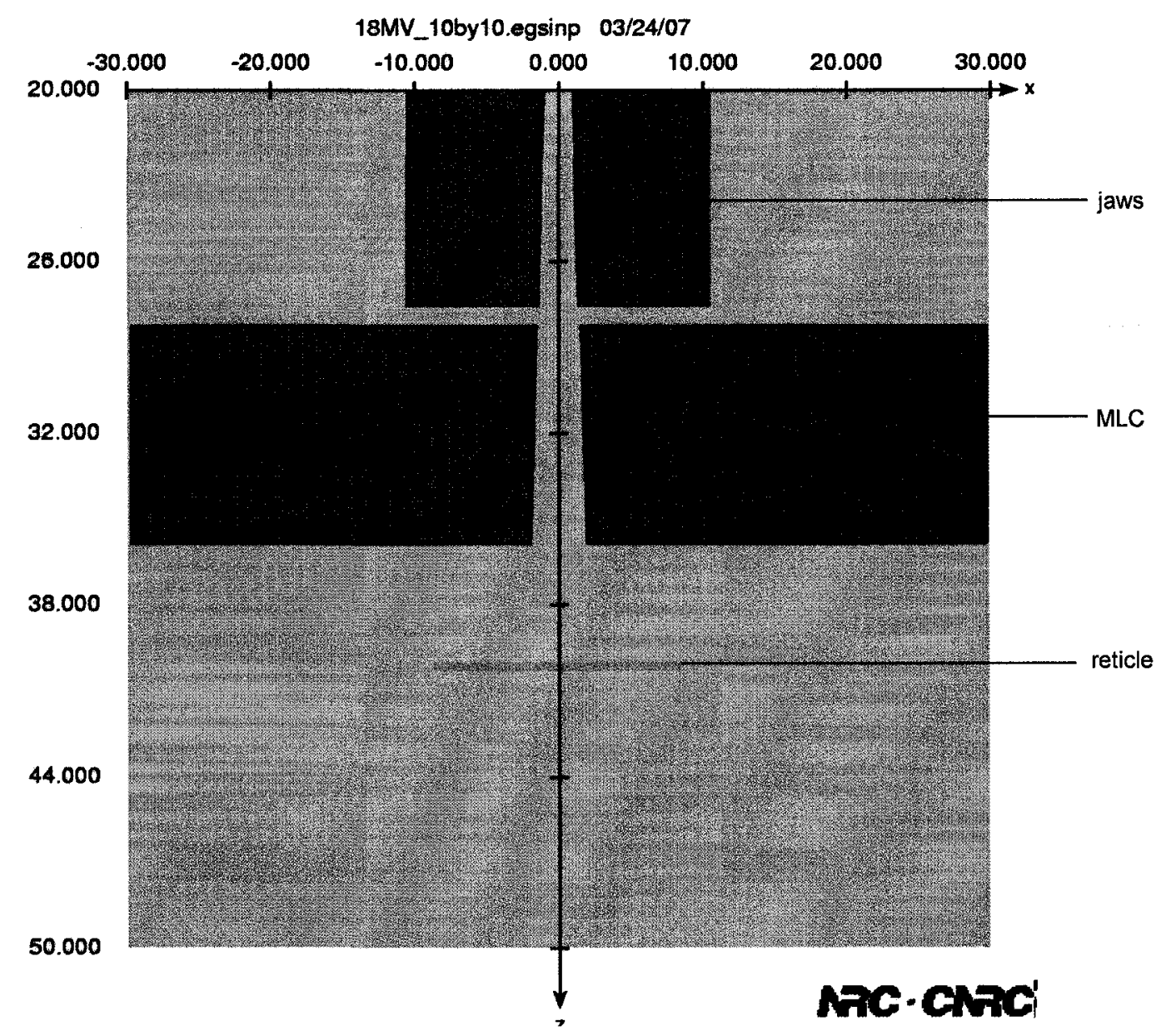

Figure 2.2 A schematic diagram of the secondary collimators and reticle components of the Siemens Mevatron beam head. The MLC leaves are parallel to the $y$-axis and are not visible in this xz-plane. These components comprise the second section of our beam model. (Diagrams provided by BEAMnrc accelerator preview function) 


\subsection{EGSnrc simulation parameters}

There are numerous simulation parameters in the EGSnrc system that can be adjusted depending on the desired focus of the results. Adjustment of these parameters is often a balance between the accuracy of the results and computational efficiency. In the EGSnrc/BEAMnrc system, AP and AE are the lowest total energies for the production of secondary Bremsstrahlung photons and electrons, respectively. When these parameters are set to lower energies, more interactions are simulated and the energy distribution is considered more realistic. However, since low energy electron transport constitutes a major part of the total CPU time, the simulation's efficiency decreases significantly (Rogers et al 1995). PCUT and ECUT represent the low-energy thresholds for photon and electron transport. Thus, when a particle's energy reaches these thresholds, its energy is considered to be deposited locally. For our beam model, the parameters were set as suggested by Rogers et al in 2001 and Sheikh-Bagheri and Rogers in 2002: AP = $\mathrm{PCUT}=0.010 \mathrm{MeV}$ and $\mathrm{AE}=\mathrm{ECUT}=0.700 \mathrm{MeV}$.

All simulations began with $2 \times 10^{7}$ original electron histories incident on the target. To increase the computational efficiency of the simulations, a variance reduction technique called range rejection was employed. This method calculates the range of the charged particle and forces it to deposit all of its energy locally if it is unable to leave the region with a pre-specified energy (Rogers et al 2002). When range rejection is applied, an approximation is made that potential bremsstrahlung photons that may have been formed by the charged particle are deposited locally as well. For this reason, the parameter ESAVE was created to minimize the inaccuracies resulting from the 
approximation. ESAVE sets the maximum charged particle energy at which range rejection can occur and was set to $2 \mathrm{MeV}$ for both 6- and 18-MV photon beams (Rogers et al 2002, Sheikh-Bagheri et al 2000).

Photon forcing is a variance reduction technique that forces photons to interact in a specific component module. Though this is useful when examining scattered photons where photon interactions are rare (such as in low-density materials), it was not considered beneficial for our beam model and was not used. Selective bremsstrahlung splitting (SBS) was utilized to improve the statistics of the bremsstrahlung photons created from electron interactions. In recent BEAMnrc implementations, SBS has been superseded by directional bremsstrahlung splitting (DBS), which offers even greater efficiency. However, this technique was not available for this work. Rogers et al (1995) reported that modeling a linear accelerator beam with bremsstrahlung splitting could increase the efficiency by a factor of 4 or more. The minimum $\left(\mathrm{N}_{\min }\right)$ and maximum $\left(\mathrm{N}_{\max }\right)$ bremsstrahlung splitting numbers were set to 25 and 250 , respectively, and Russian Roulette was switched on since good statistics for both photons and electrons is needed for accurate dose distributions. Typical calculation times to develop the beam model varied between 15 to 25 hours per simulation on a $2.4 \mathrm{GHz}$ Pentium processor. Typical experiments utilizing the beam model varied between 10 to 20 hours, depending on experimental parameters. 


\subsection{Composition of materials}

Conventional treatment planning algorithms are based on electron density information provided by the CT dataset. The accuracy of the dose calculations is often limited by the approximations made in modeling the secondary electron transport. Monte Carlo dose calculation algorithms model the particle transport explicitly. For this reason, it is important to provide the $\mathrm{MC}$ simulation with accurate atomic composition data on any materials used in the beam model.

The EGSnrc system relies on a stand-alone utility program called PEGS4 (preelectron gamma shower) whose purpose is to provide material data for the EGS code (Kawrakow and Rogers 2006). One of the most important pieces of data provided by PEGS4 is the material-specific and energy-dependent cross section data. BEAMnrc provides a default list of materials and PEGS4 data for AE (lower energy threshold for the production of secondary electrons) energies ranging between $0.700 \mathrm{MeV}$ to $55 \mathrm{MeV}$, and based on density effect corrections in ICRU Report 37 (ICRU 1984). These materials were used as the basis for our simulations and are shown in Table 2.1. Additional materials specific to the linear accelerator's manufacturer were generated individually and subsequently added to the default list. These additional materials, their densities and percentage composition by weight (when appropriate) are shown in Table 2.2. 
Table 2.1 BEAMnrc defined (default) materials with cross-sectional data calculated by PEGS4, based on density effect corrections in ICRU Report 37.

\begin{tabular}{|l|l|}
\hline \multicolumn{2}{|c|}{ BEAMnrC defined materials } \\
\hline AG700ICRU & NI700ICRU \\
\hline AIR700ICRU & NYLON6AND66700ICRU \\
\hline ALUMINA700ICRU & PB700ICRU \\
\hline AL700ICRU & PBSBICU700 \\
\hline AU700ICRU & PMMA700ICRU \\
\hline BE700ICRU & POLYETH700ICRU \\
\hline CERROBEND700 & POLYSTY700ICRU \\
\hline CU700ICRU & SI700ICRU \\
\hline FE700ICRU & SIO2700ICRU \\
\hline GE700ICRU & SN700ICRU \\
\hline 170C700ICRU & STEEL700ICRU \\
\hline 226C700ICRU & STEEL_8P5_700 \\
\hline H2O700ICRU & TA700ICRU \\
\hline HE700ICRU & T1700ICRU \\
\hline ICRPBONE700ICRU & W700ICRU \\
\hline ICRUTISSUE700ICRU & WNICU700 \\
\hline KAPTON700ICRU & WRE700 \\
\hline LUNG700ICRU & W16P9_700 \\
\hline MG700ICRU & WRE_19P4_700 \\
\hline MICA700 & WRE_18P0_700 \\
\hline MILDSTEEL700 & YELBRASS700ICRU \\
\hline MYLAR700ICRU & ZN700ICRU \\
\hline NAI700ICRU & \\
\hline
\end{tabular}


Table 2.2 Additional mixtures, compounds, and elements specific to the Siemens Mevatron linear accelerator. This information was entered into PEGS4 to produce crosssectional data for the EGSnrc / BEAMnrc beam model simulations.

\begin{tabular}{|c|c|c|c|}
\hline Material & $\frac{\text { Density }}{\text { (g/cm3) }}$ & Element & $\frac{\% \text { Composition }}{\text { (by weight) }}$ \\
\hline \multirow{8}{*}{ Stainless Steel 303} & \multirow{8}{*}{8.19} & Carbon & 0.100 \\
\hline & & Manganese & 2.000 \\
\hline & & Phosphorus & 0.045 \\
\hline & & Sulphur & 0.030 \\
\hline & & Silicon & 1.000 \\
\hline & & Chromium & 18.000 \\
\hline & & Nickel & 9.000 \\
\hline & & Iron & 69.825 \\
\hline \multirow{6}{*}{ Stainless Steel 304} & \multirow{6}{*}{8.19} & Carbon & 0.050 \\
\hline & & Manganese & 2.000 \\
\hline & & Silicon & 1.000 \\
\hline & & Chromium & 19.000 \\
\hline & & Nickel & 9.500 \\
\hline & & Iron & 68.450 \\
\hline \multirow{3}{*}{ Lexan 9034} & \multirow{3}{*}{1.20} & Carbon & 75.600 \\
\hline & & Hydrogen & 5.500 \\
\hline & & Oxygen & 18.900 \\
\hline $\begin{array}{c}\text { Alumina } \\
\left.\text { (Wesgo } \mathrm{Al}-995, \mathrm{Al}_{2} \mathrm{O}_{3}\right)\end{array}$ & 6.09 & & \\
\hline Tungsten & 19.3 & & \\
\hline Silicon Dioxide $\left(\mathrm{SiO}_{2}\right)$ & 2.50 & & \\
\hline
\end{tabular}




\subsection{Optimizing beam model parameters}

In radiotherapy clinics, some of the most important linear accelerator parameters are difficult to obtain accurately. These parameters include the initial electron beam energy and the radial intensity distribution of the incident beam. However, manufacturers recommend that these specifications for the initial electron beam are only guidelines and have a large uncertainty. In addition, clinical linear accelerators are often tuned upon commissioning, changing the energy of the electron beam incident on the target.

Determining these parameters is an iterative process and begins by running the simulation using the specifications supplied by the manufacturer. This data was then compared to measured central axis depth-dose curves and lateral dose profiles at specific depths obtained during the commissioning of the linear accelerator. The incident electron mean energy was adjusted until there was sufficient agreement with the depth-dose curves. Once this has been achieved, the FWHM (full-width half-maximum) of the radial intensity distribution is adjusted until both the depth-dose curve and the lateral dose profile provide a good match with the measured data. Unfortunately, this match is not easily obtainable and often requires one to go back and repeatedly fine-tune the incident electron energy. Thus, the parameter optimization process requires a large number of iterations and lengthy computing time.

The standard procedure for determining the FWHM of the radial intensity is to examine the impact any changes have on broad beam data. If the variables were optimized to give an accurate profile for a large field, then they were likely to agree with 
measured data for smaller fields. Initially, the dose profiles of $30 \times 30 \mathrm{~cm}^{2}$ fields were examined, adjusting the parameters accordingly. The measured data for such large field sizes show "horns" in the dose profiles. These characteristic "horns" represent the elevated shoulder regions in the dose profile of large photon fields. Unfortunately, after extensive adjustment of the spot size, the simulated dose profile was never able to demonstrate these horns. Further investigation on this matter will be considered in future studies to solidify our MC beam model. Due to time constraints, it was decided to model the beam based on a smaller $10 \times 10 \mathrm{~cm}^{2}$ field since this would be the field size used in our future experiments.

The initial electron beam energies specified by the manufacturer were 6.70 and $14.28 \mathrm{MeV}$ for the 6 and $18 \mathrm{MV}$ photon beams, respectively. It has been shown that offaxis factors are sensitive to both the mean energy of the electron beam and its energy distribution, especially for higher energy photon beams (Sheikh-Bagheri and Rogers 2002). The manufacturer recommended distribution is similar to a Laurentzian, falling off sharper past the peak. This was approximated by an asymmetrical Gaussian distribution, with a FWHM of $14 \%$ on the low-energy side and a FWHM of $3 \%$ on the high-energy side (as recommended by Sheikh-Bagheri and Rogers in 2002). For the 6 MV beam, providing an energy distribution rather than a single-energy electron beam for the MC simulations showed little difference in the off-axis factors. An energy distribution with a peak energy of $6.88 \mathrm{MeV}$ most accurately simulated the measured data. Using a single-energy electron beam for the $18 \mathrm{MV}$ beam resulted in a drooping of the shoulders of the dose profile when compared to measured data. This effect improved 
with the application of the asymmetrical Gaussian energy distribution. Figure 2.3 shows a sample of the energy distributions examined for the $18 \mathrm{MV}$ photon beam model. The Siemens Primus 18 MV photon beam in our clinic had been tuned down significantly at the time of commissioning, behaving more like a $15 \mathrm{MV}$ beam than an $18 \mathrm{MV}$ beam. It was found that an energy distribution peaking at $14.55 \mathrm{MeV}$ and with an average energy of $14.1 \mathrm{MeV}$ most closely depicted the measured depth dose and profile data.

The spot size of the initial electron beam plays a large role in the shape of the dose profile. Following recommendations by Faddegon et al (2005), Sheikh-Bagheri and Rogers (2002), and Ding (2002), a spot size with a Gaussian radial intensity distribution and a FWHM of $0.1 \mathrm{~cm}$ was used as a starting reference. Numerous spot sizes were examined but a comparison of measured and simulated data confirmed that a FWHM of $0.05 \mathrm{~cm}$ was the most appropriate for our beam model. BEAMnrc provides models for many radiation sources with varying geometries and distributions to suit the user's experimental needs and individual linear accelerator specifications. Two such sources examined in our beam model development were: 1) a circular parallel beam with a 2-D Gaussian distribution and 2) an isotropic point source on the z-axis. In the latter, the angular spread of the electron beam was adjusted to varying degrees, but ultimately, it was found that the circular parallel beam with 2-D Gaussian distribution provided the most accurate results.

A comparison between the Monte Carlo calculated and measured central axis depth-dose curves for a $6 \mathrm{MV}, 10 \times 10 \mathrm{~cm}^{2}$ photon beam incident on a $30 \times 30 \times 30 \mathrm{~cm}^{3}$ 
homogeneous water phantom $(100 \mathrm{~cm} \mathrm{SAD})$ is shown in Figure 2.4. The beam profiles at three depths are shown in Figure 2.5. Similar comparisons for the $18 \mathrm{MV}$ beam model are shown in Figures 2.6 and 2.7. The depth-dose curves were predominantly responsible for estimating the incident electron energy, and were quite insensitive to other changes in the beam model parameters. Conversely, the "shoulder" regions of the beam profiles were used extensively to optimize the beam model. All MC dose calculations were in excellent agreement with the measured data for $10 \times 10 \mathrm{~cm}^{2}$ photon beams. 
18MV Incident Electron Spectra

(asymmetrical Gaussian with FWHM $=14 \%$ \& 3\%)

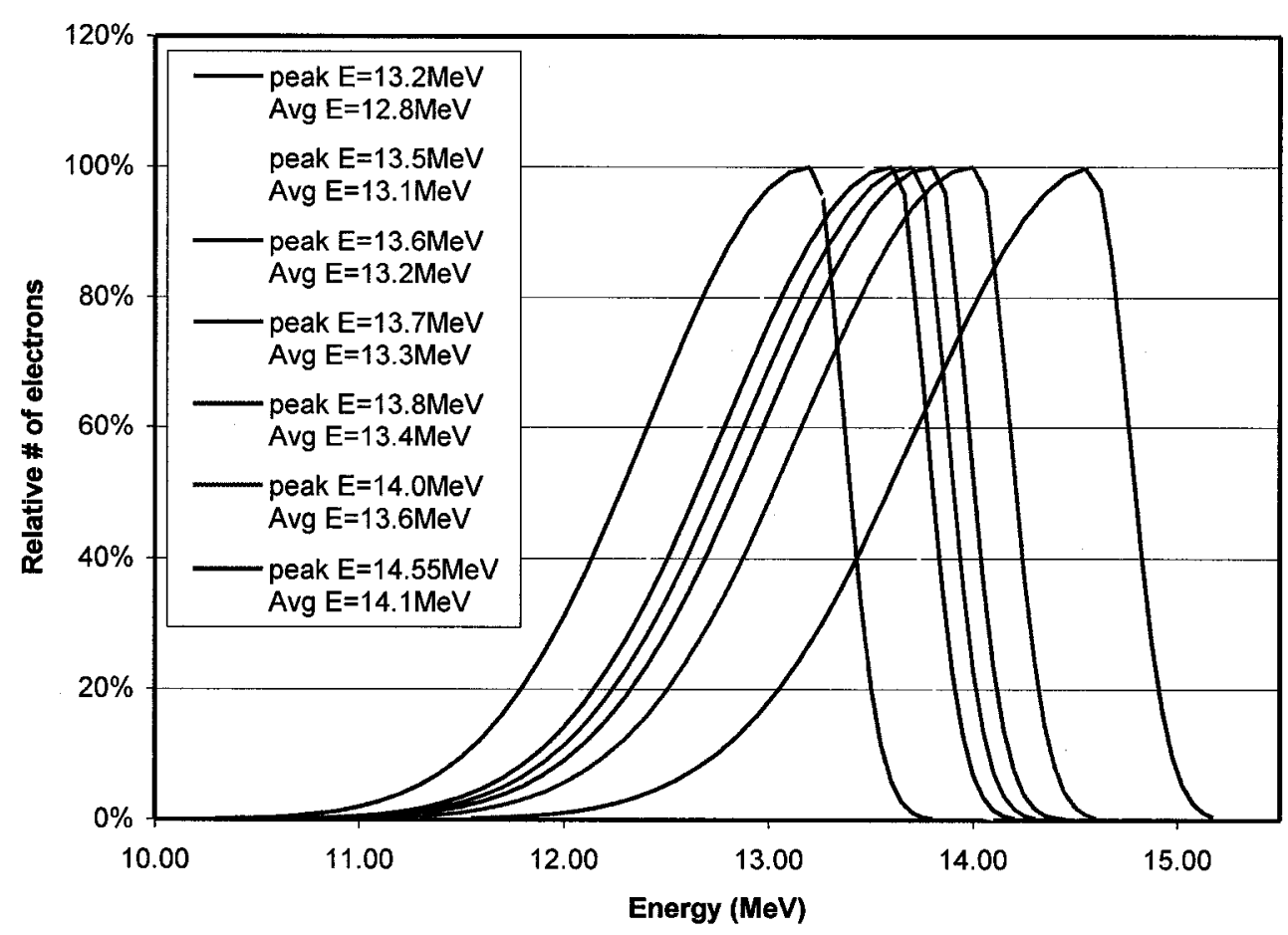

Figure 2.3 Incident electron energy spectra examined for the optimization of the

Siemens Primus linear accelerator treatment head for an $18 \mathrm{MV}$ beam modeled by BEAMnrc. An asymmetrical Gaussian distribution was used as suggested by SheikhBagheri and Rogers (2002). 


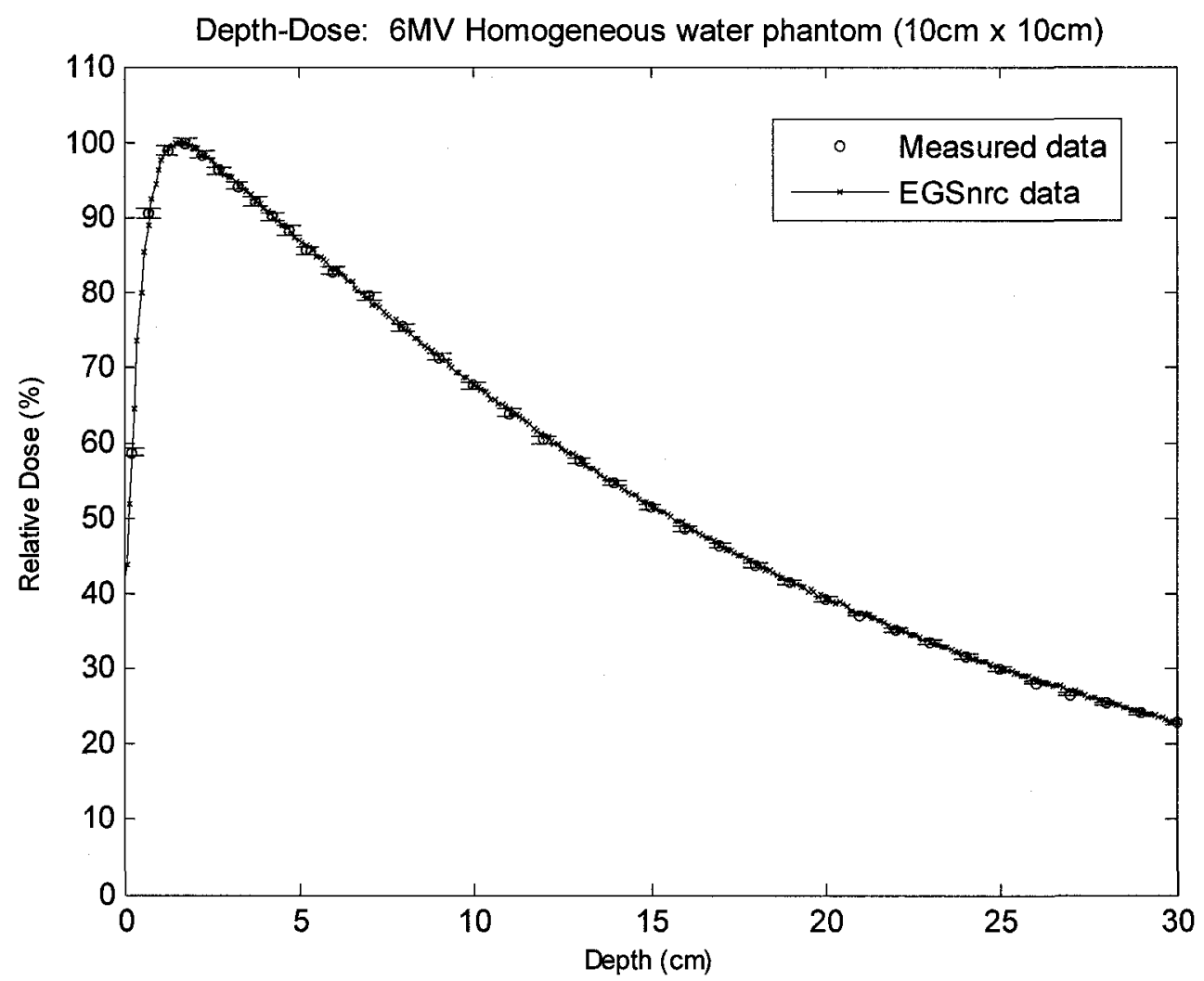

Figure 2.4 Central axis depth-dose curves in a homogeneous water phantom for a $6 \mathrm{MV}$, $10 \times 10 \mathrm{~cm}^{2}$ field measured with diodes and modeled using the EGSnrc/BEAMnrc/DOSXYZnrc system. 


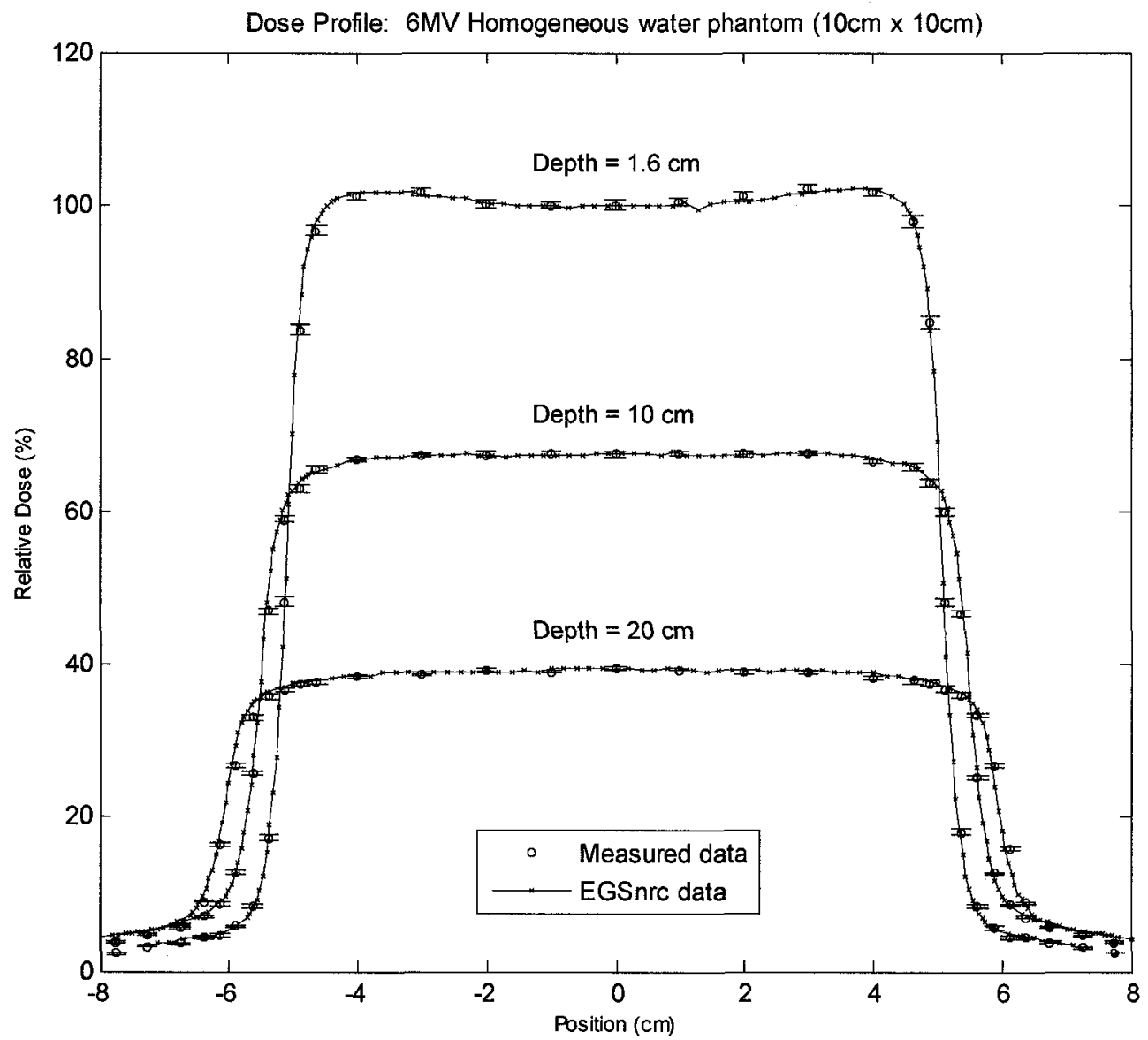

Figure 2.5 Beam profiles at depths of 1.6, 10, and $20 \mathrm{~cm}$ in a homogeneous water phantom for a $6 \mathrm{MV}, 10 \times 10 \mathrm{~cm}^{2}$ field measured with diodes and modeled using the EGSnrc/BEAMnrc/DOSXYZnrc system. 


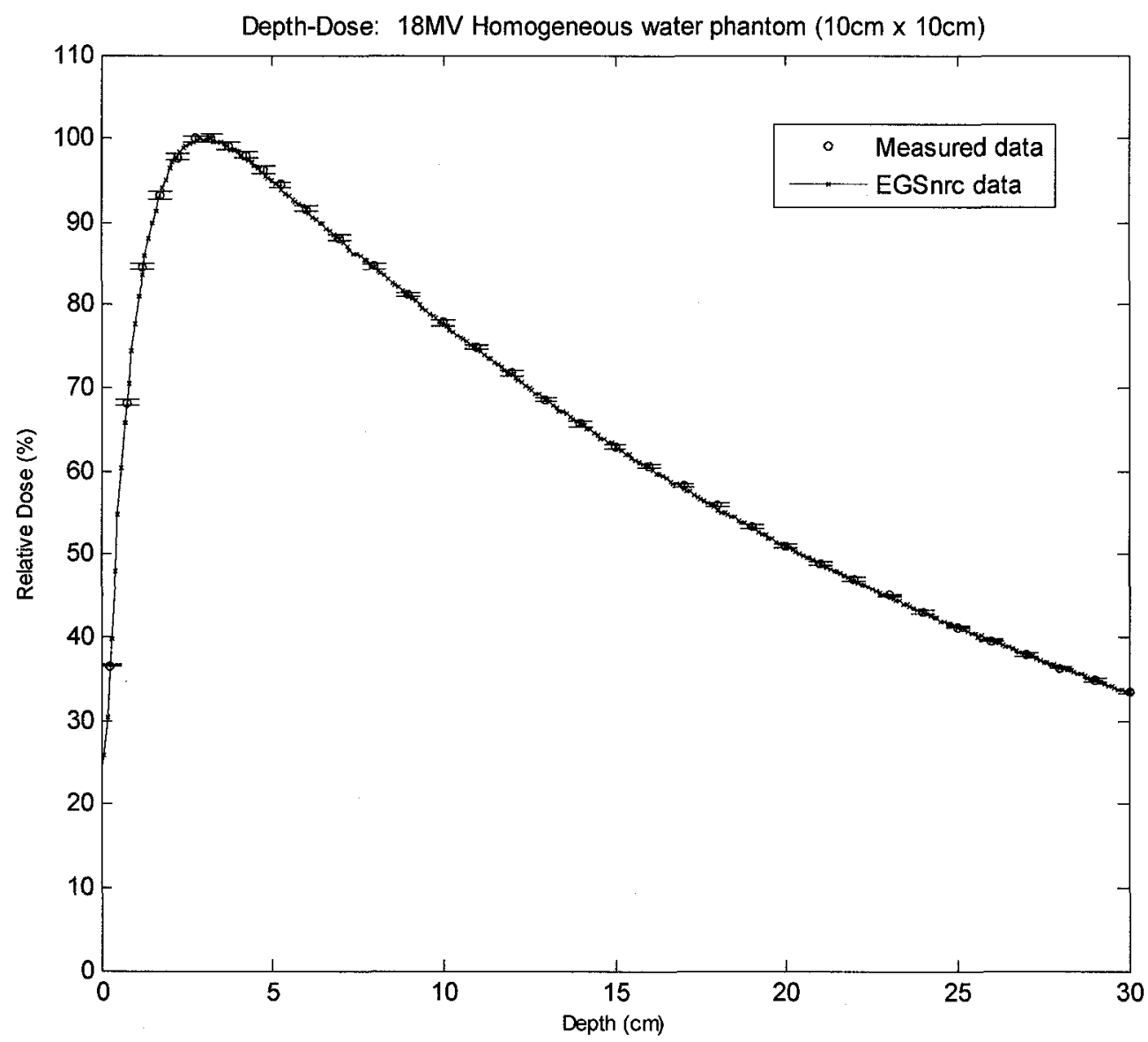

Figure 2.6 Central axis depth-dose curves in a homogeneous water phantom for an 18 MV, $10 \times 10 \mathrm{~cm}^{2}$ field measured with diodes and modeled using the EGSnrc/BEAMnrc/DOSXYZnrc system. 


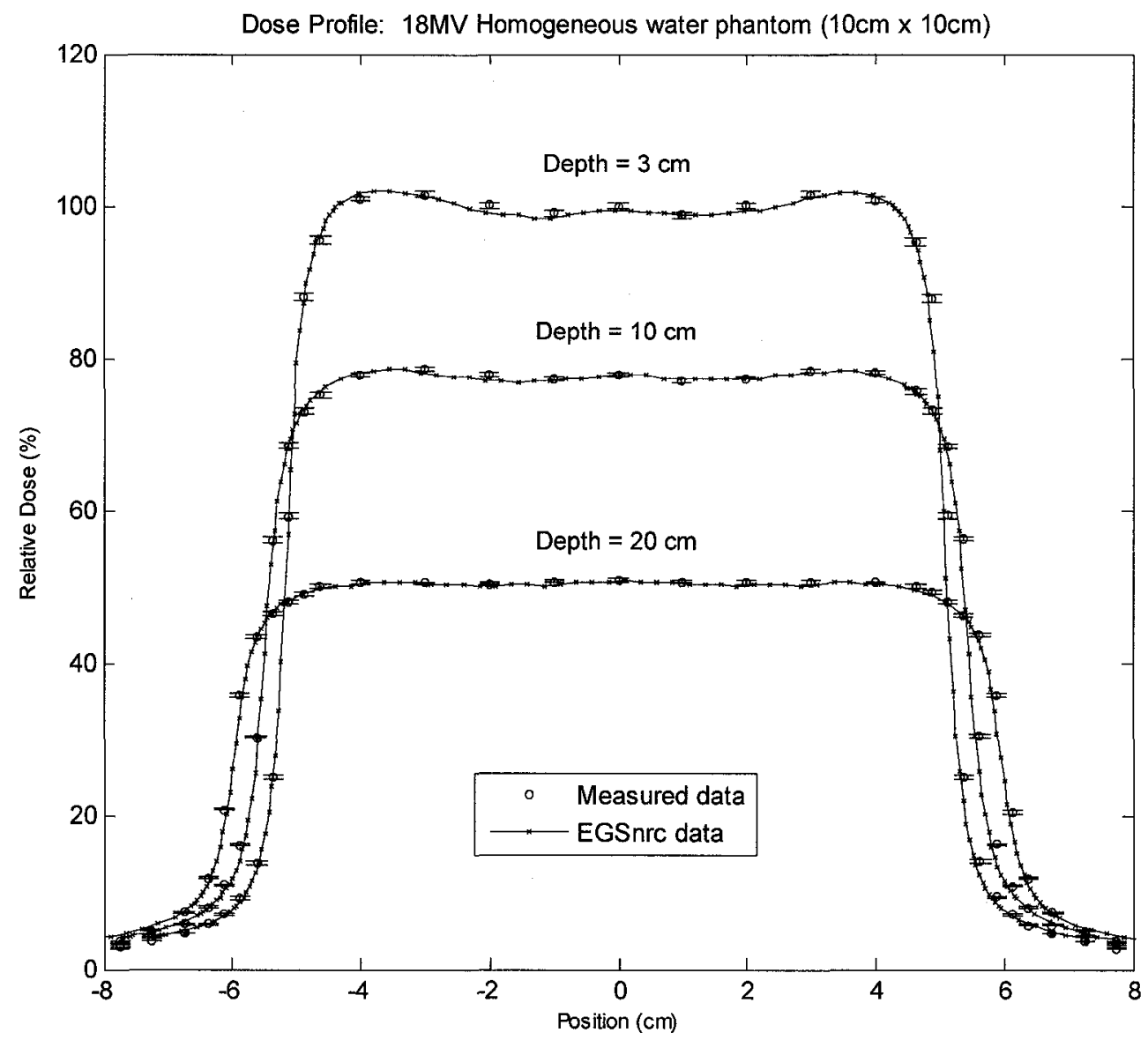

Figure 2.7 Beam profiles at depths of $1.6,10$, and $20 \mathrm{~cm}$ in a homogeneous water phantom for an $18 \mathrm{MV}, 10 \times 10 \mathrm{~cm}^{2}$ field measured with diodes and modeled using the EGSnrc/BEAMnrc/DOSXYZnrc system. 


\subsubsection{Monitor unit conversion}

Clinical radiotherapy linear accelerators use transmission type ionization chambers to monitor the radiation output during delivery. The prescribed absorbed dose to a patient is converted to the readings of the monitor chamber on a treatment unit or the monitor unit (MU). The treatment units at the Ottawa Hospital Regional Cancer Centre are calibrated to give $1 \mathrm{MU}$ per cGy for a $10 \times 10 \mathrm{~cm}^{2}$ field at $100 \mathrm{SAD}$ and $5 \mathrm{~cm}$ depth in a water phantom. The absorbed dose at all other points in the water phantom can be related to the reference point through central-axis depth-dose curves, dose profiles at various depths, and output and assorted beam correction factors.

BEAMnrc/DOSXYZnre provides dose calculations in units of "cGy per incident electron". The DOSXYZnrc calculated dose was calibrated by simulating the reference geometry in a virtual water phantom. For each beam model, a fixed number of incident electrons are simulated in each experiment. Under the reference conditions:

$$
\text { (\# of incident electrons/cGy) }=(\# \text { of incident electrons/MU) }
$$

and the absorbed dose was determined by applying the following conversion:

$$
\left(\frac{c G y}{e^{-}}\right)_{D O S x y z n r c} \times\left(\frac{e^{-}}{M U}\right)_{\text {reference }} \times M U=c G y
$$

where the first term represents the dose output provided by DOSXYZnrc, the second term is dose calibration factor (DCF) under reference conditions, and MU is the number of monitor units applied in the experiment. Table 2.3 lists the DCFs used in our simulations for 6 and $18 \mathrm{MV}$ photon beams models. 
It should be noted that Verhaegen et al (2000) showed that in a Varian $2100 \mathrm{C}$ linear accelerator, a portion of the charge collected by the monitor chamber is backscattered from components downstream. The output of the accelerator is affected and must be taken into account in Monte Carlo derived output factors. Verhaegen et al's results demonstrated linear increase in the backscatter fraction for decreasing field sizes. For example, a $6 \mathrm{MV}$ photon beam was measured to have a $3 \%$ increase in backscatter when comparing a $0.5 \times 0.5 \mathrm{~cm}^{2}$ field with a $40 \times 40 \mathrm{~cm}^{2}$ field. The same comparison for a $10 \mathrm{MV}$ photon beam showed a $2 \%$ increase in backscatter. Therefore, it is recommended that a correction factor of up to 2 to $3 \%$ be applied to output factors ( $\mathrm{e}^{-}$per $\mathrm{MU}$ ) created by Monte Carlo simulations for smaller fields compared to larger fields. These corrections were not taken into account in our output factors and need to be considered for the experiments reported in Chapter 5. 
Table 2.3 The dose calibration factors used to convert BEAMnrc/DOSXYZnrc dose outputs (cGy per incident electron) to units of cGy/MU.

\begin{tabular}{|c|c|}
\hline Photon Beam Energy (MV) & $\begin{array}{c}\text { Dose calibration factor } \\
\text { (electrons/MU) }\end{array}$ \\
\hline 6 & $7.373 \times 10^{15}$ \\
\hline 18 & $1.593 \times 10^{15}$ \\
\hline
\end{tabular}




\section{Chapter 3}

\section{Treatment planning dose calculations in low $\mathrm{Z}$ materials}

\subsection{Introduction}

In external beam radiotherapy, the aim of treatment planning systems (TPSs) is to provide dose calculations accurately and efficiently for a variety of beam geometries. This balance between accuracy and efficiency, along with technological advances in computational speed, has guided the development of TPSs. Some of the largest errors in the predicted dose arise in the proximity of tissue inhomogeneities. Tissues such as lung, the oral cavities, nasal passages, sinuses and bone are of particular interest because their radiological properties differ from water or normal tissue. This chapter will examine the ability of two algorithms (Pencil Beam (PB) and Collapsed-Cone Convolution (CCC) techniques) implemented on a commercial radiotherapy TPS to calculate dose in lungequivalent material. The TPS investigated in this study was Theraplan Plus version 3.8 with Oncentra DCM version 2.0 (MDS Nordion, Ottawa, ON, Canada). 
In the $1980 \mathrm{~s}$, multiple studies quantified the dosimetric consequences of lung in radiotherapy treatment fields (Beach et al 1987, El-Khatib and Battista 1984, El-Khatib and Battista 1986, Mackie et al 1985). Beach et al compared the effect of air-cavity inhomogeneities for $\mathrm{Co}^{60}, 6 \mathrm{MV}$, and $10 \mathrm{MV}$-ray beams and attributed the dosimetric complications to a loss of charged particle equilibrium in tissues near the air-tissue interfaces. In 1984 and 1986, El-Khatib and Battista proposed correction methods for computing dose in the presence of lung inhomogeneities. Mackie et al (1985) also examined the accuracy of various lung dose correction techniques for 6 and $15 \mathrm{MV}$ xrays. Subsequently, lung dose correction algorithms have continued to develop to keep up with improvements in TPSs, increased computing power, and advances in highprecision treatment techniques such as IMRT (Behrens 2006, Blomquist and Karlsson 1998, Engelsman et al 2001, Essers et al 1995, Essers and Mijnheer 1999, Klein et al 1997, Mah and Van Dyk 1991, Orton et al 1998, Papanikolaou and Klein 2000, Petoukhova et al 2006).

Modern TPSs range in their ability to explicitly model electron transport. Monte Carlo based dose calculation algorithms model the transport of each particle and hence, no inhomogeneity corrections need to be applied. However, these algorithms are computationally expensive for photon radiotherapy and are not commonly employed in the clinic. Most clinical TPSs utilize semi-analytic algorithms that make approximations for the particle transport and apply corrections in the presence of tissue inhomogeneities. Common examples of such algorithms are the collapsed-cone convolution and pencilbeam techniques. There are numerous lung dose verification and validation studies 
regarding not only specific dose calculation algorithms but also the implementation of these algorithms in commercial TPSs (Aspradakis et al 2003, Butson et al 2000, Carrasco et al 2004, Cranmer-Sargison et al 2004, Gagné and Zavgorodni 2007, Knöös et al 2006, Kron et al 2004, Laub et al 2001, Laub and Nüsslin 2003, Ramsey et al 1999, Weber and Nilsson 2002). Alam et al (1997) applied the AAPM Radiation Therapy Committee Task Group 23 test package (developed for the verification of the accuracy of TPSs) to two commercial TPSs, including Theraplan version 5, for photon external beam radiotherapy. However, this version of Theraplan utilizes the scatter integration model developed by Cunningham (1972) rather than the pencil-beam algorithm implemented in Theraplan Plus version 3.8. Chow et al (2003) examined the accuracy of the PB and collapsed-cone convolution algorithms implemented in Theraplan Plus version 3.7 for photon arcs in the presence of a lung inhomogeneity. For a $90^{\circ} 18 \mathrm{MV}$ photon arc with 4 $\times 4 \mathrm{~cm}^{2}$ and $10 \times 10 \mathrm{~cm}^{2}$ field sizes, the PB algorithm overestimated the dose by approximately $12 \%$ and $6 \%$, respectively, in the middle of the lung. The results for the same setup with the collapsed cone convolution algorithm showed good agreement with measurements for the $10 \times 10 \mathrm{~cm}^{2}$ field but a $6 \%$ underestimation of the dose in the middle of the lung for the $4 \times 4 \mathrm{~cm}^{2}$. However, this study focused on photon arc therapy rather than single beams. Furthermore, its beam profiles were limited to a single depth in the middle of the lung material.

This aim of this study is to quantify the lung dose calculation errors in the PB and collapsed cone convolution algorithms as they have been implemented in Theraplan Plus version 3.8 with Oncentra DCM version 2.0. This chapter will begin with a review of the 
pertinent dose calculation algorithms utilized in this thesis. The subsequent sections will introduce and discuss the lung dose measurements and calculations performed in a lung phantom. It should be noted that both the PB and CCC algorithms implemented by Helax-TMS TPS have been examined for their accuracy in lung inhomogeneities (Haedinger et al 2005). These algorithms are thought to be the same as those used in Theraplan Plus. The goal of this work is to ensure that the algorithms were implemented correctly in Theraplan Plus.

\subsection{Dose calculation algorithms}

The earliest treatment planning calculations for high-energy beams were based on empirical methods. At the time, the largest limitation to the accuracy of the dose calculations was the lack of patient information. The development of faster computer processing capabilities and imaging modalities, such as CT, MRI and PET, increased the need for more accurate dose calculation algorithms. Empirical methods are limited to simple geometries and are often inaccurate. Analytical methods have potential for predicting simple processes and events but are unable to accurately track the extensive histories created by an electron shower. Conversely, MC methods model the transport of each particle until all of its energy is absorbed or escapes. The main limitation to MC based TP systems has been computing time. Though there have been significant advances in this area, MC based dose calculation algorithms for photon beams have yet to become a regular fixture in clinical TP systems. Thus the current standard is a semianalytical model, such as convolution/superposition methods, that incorporate presimulated MC based dose calculations. In general, it has become evident that the more 
explicitly the charged particle transport is modeled, the greater the accuracy of the dose calculation algorithm.

\subsubsection{Convolution/superposition models}

Convolution/superposition algorithms utilize energy deposition kernels (EDKs) where the dose deposition is represented by a superposition of weighted responses (kernels) to point irradiations. The use of kernels in radiotherapy stems from the dose deposition process of photon beams incident on patients. The primary attenuation of the photon beam can be calculated from the beam spectrum and knowledge of the patient's composition. This also gives rise to the amount of radiant energy locally released in the patient. As discussed in previous sections, this energy is distributed by secondary particles resulting in absorbed dose. This process of energy release and deposition was modeled as a convolution of the primary photon energy distribution with an EDK (Ahnesjö et al 1987, Boyer and Mok 1985, Mackie et al 1985).

The kernel represents the energy transported and deposited by secondary particles. The most practical means of calculating EDKs are with Monte Carlo particle transport simulations, as done by Ahnesjö et al (1987), Mackie et al (1988) and Mohan et al (1986). Generally, EDKs are categorized into three types that describe the geometry of the incident beam from which they were formed (Wang et al 1995). The first is the point kernel that describes the pattern of energy deposition in an infinite medium from a primary photon interaction. The second is the pencil beam kernel that arises from the energy deposition pattern in a semi-infinite medium from a point mono-directional 
photon beam. Finally, the third is the planar kernel that represents the energy spread from an infinitely broad photon beam.

Kernels are often separated into primary $\left(h_{p}\right)$ and phantom scatter $\left(h_{s}\right)$ dose and are closely linked to the energy absorption coefficients (Mackie et al 1988). It has been shown that:

$$
\iiint_{\infty} h_{p}(\vec{r}) d x d y d z=\frac{\mu_{e n}}{\mu}
$$

and

$$
\iiint_{\infty} h_{s}(\vec{r}) d x d y d z=\frac{\mu-\mu_{e n}}{\mu}
$$

where $\mu_{e n}$ is the energy absorption coefficient and $\mu$ is the linear attenuation coefficient. However, it is difficult to use these analytical expressions for calculating dose kernels because of the complex nature of particle transport. Thus, efforts were made to analytically model and fit the primary and scatter dose kernels (Ahnesjö 1989, Ahnesjö et al 1992, Ahnesjö and Mackie 1987, Nilsson and Knöös 1992, Wang et al 1995). In 1989, Ahnesjö found a good fit to poly-energetic point kernels:

$$
h(\vec{r})=\frac{A_{\theta} e^{-a_{\theta} r}+B_{\theta} e^{-b_{\theta} r}}{r^{2}}
$$


where $A_{\theta}, B_{\theta}, a_{\theta}$ and $b_{\theta}$ are fitting parameters dependent on the scattering angle $\theta$ and $r$ is the absolute value of the vector $\vec{r}$. The first and second terms in the equation represent the primary and scatter dose components, respectively.

The dose is calculated from point kernels by first calculating the attenuation of the primary photons or the energy transferred to secondary particles (total energy released per unit mass, TERMA), and then secondly to convolve this with weighted dose spread kernels. For a monoenergetic parallel beam irradiating a homogeneous phantom, the total dose is as follows:

$$
D(\vec{r})=\iiint_{V} T(\vec{s}) h(\vec{r}-\vec{s}) d^{3} s
$$

where $T(\vec{s})$ is the TERMA in the volume element $d^{3} s$ and $h(\vec{r}-\vec{s})$ represents the dose spread kernel that describes the mean fraction of energy deposited per unit volume at $\vec{r}$ per photon interacting at $\vec{s}$. Equation 3.4 can be generalized for polyenergetic beams by incorporating an energy-dependent kernel and TERMA variable:

$$
D(\vec{r})=\int_{E} \iiint_{V} T_{E}(\vec{s}) h(E, \vec{r}-\vec{s}) d^{3} s d E
$$

where

$$
T_{E}(\vec{s})=\frac{\mu}{\rho}(E, \vec{s}) \Psi_{E}(\vec{s})
$$

and $\frac{\mu}{\rho}(E, \vec{s})$ is the mass-attenuation coefficient of the primary photons of energy $E$ and $\Psi_{E}(\vec{s})$ is the energy fluence of primary photons at $\vec{s}$ (Ahnesjö and Aspradakis 1999). In 1994, Hoban et al showed that equation 3.5 could accurately be separated into primary 
$(P(\vec{s}))$ and scatter $(S(\vec{s}))$ dose convolutions, allowing for individual examination of each component. The new dose equation was expressed as:

$$
D(\vec{r})=\iiint_{V} P(\vec{s}) \tilde{h}_{p}(\vec{r}-\vec{s}) d^{3} s+\iiint_{V} S(\vec{s}) \widetilde{h}_{s}(\vec{r}-\vec{s}) d^{3} s
$$

where

$$
\begin{gathered}
P(\vec{s})=\int T_{E}(\vec{s}) \frac{\mu_{e n}(E)}{\rho} d E \\
S(\vec{s})=\int T_{E}(\vec{s})\left(1-\frac{\mu_{e n}(E)}{\rho}\right) d E \\
\widetilde{h}_{p}(\vec{r})=\frac{\int \Psi_{E}\left(z_{0}\right) \mu(E) h_{p}(E, \vec{r}) d E}{\int \Psi_{E}\left(z_{0}\right) \mu_{e n}(E) d E} \\
\widetilde{h}_{s}(\vec{r})=\frac{\int \Psi_{E}\left(z_{0}\right) \mu(E) h_{s}(E, \vec{r}) d E}{\int \Psi_{E}\left(z_{0}\right)\left(\mu(E)-\mu_{e n}(E)\right) d E}
\end{gathered}
$$$$
\text { and }
$$

where the primary and scatter kernels are defined and renormalized at a specific depth $\mathrm{z}_{0}$.

In the presence of tissue inhomogeneities, the common approach is to scale the dose of each kernel with the relative electron density between point $s$ defined by $\vec{s}$ (photon interaction site) and point $r$ define by $\vec{r}$ (energy deposition site). The argument behind this method is that the scattering cross section is largely in the forward direction, and therefore the transfer of energy has a stronger dependence on the initial site of photon interaction rather than at the sites of multiply scattered particles. The dose kernel in heterogeneous media can be written as follows: 


$$
h_{h e t}(\vec{s}, \vec{r})=\frac{\rho(\vec{r})}{\rho_{0}} c^{2} h_{\rho_{0}}[c(\vec{r}-\vec{s})]
$$

where

$$
c=c(\vec{s}, \vec{r})=\int_{0}^{l} \rho_{r e l}[\vec{s}-\ell \cdot(\vec{s}-\vec{r})] d \ell
$$

$h_{\rho_{0}}$ represents the dose point kernel calculated for a homogeneous medium of mass density $\rho_{0}$, and $\rho_{r e l}$ is the electron density of the medium at the interaction site relative to the reference medium (water). $\ell \cdot(\vec{s}-\vec{r})$ represents the path between the photon interaction and dose deposition sites. Incorporating equation 3.12 into the dose equation (Equation 3.4) results in the following superposition integral that replaces the original convolution integral:

$$
D(\vec{r})=\iiint_{V} T(\vec{s}) \frac{\rho(\vec{s})}{\rho_{0}} c^{2} h_{p_{0}}[c(\vec{r}-\vec{s})] d^{3} s
$$

Approximating the dose kernels with the density scaling approach increases the calculation efficiency but it has been shown that there may be significant inaccuracies locally (Woo and Cunningham 1990). However, these local discrepancies are less evident once kernels from surrounding regions are averaged.

\subsubsection{Collapsed cone convolution}

Full implementation of the convolution/superposition method is limited by computational speed. Thus many researchers have developed efficient algorithms to decrease the total calculation time while maintaining sufficient accuracy for clinical use. In 1989, Ahnesjö developed the collapsed cone convolution (CCC) method as an approximation to the 
point kernel approach. This algorithm is provided in Oncentra DCM v2.0, an optional dose calculation method within Theraplan Plus version 3.8 (MDS Nordion, Ottawa, Canada), a TPS used throughout this thesis. This method performs an angular discretization of the dose kernel so that the kernel is divided into a number of unevenly distributed cones. Since the energy released from a primary photon interaction is predominantly in the forward direction, the number of cones allocated in the forward region is greater than those allocated laterally. Based on the analytical kernel expression (equation 3.7), the energy deposition per radial distance for each discrete cone $\Omega_{\mathrm{i}}$ can be expressed as:

$$
\iint_{\Omega_{i}}^{h_{\rho_{0}}(\vec{r}, \Omega)} \frac{r^{2}}{\rho} d^{2} \Omega=A_{\Omega_{i}} e^{-a_{\Omega_{i}} r}+B_{\Omega_{i}} e^{-b_{\Omega_{i}} r}
$$

The approximation of this technique is that the energy released within each cone is transported, attenuated, and deposited only along its axis. In other words, the cones are collapsed onto their axes, and each axis represents a transport line. All of the transport lines form a matrix with each intersection representing the center of a dose calculation voxel. The calculation speed with the CCC method is proportional to $\mathrm{M} \times \mathrm{N}^{3}$ where $\mathrm{N}^{3}$ is the number of voxels in the dose calculation volume and $\mathrm{M}$ is the number of cones in each point dose kernel.

\subsubsection{Multi-grid superposition}

The multi-grid superposition (MGS) algorithm is based on the collapsed cone convolution methodology and serves to accelerate the superposition calculation efficiency (Miften et al 2000). This algorithm is used in the XiO TPS (CMS Inc., USA) 
and is described in this chapter since it is examined in the latter chapters of this thesis. The MGS technique optimizes the number of calculation points in the dose volume by allowing for a variable resolution dose calculation matrix. In regions where higher resolution is required, such as near beam edges and tissue density gradients, the algorithm increases the density of dose calculation points. Conversely, outside the field or in regions of relative homogeneity, the dose calculation points are made sparser.

\subsubsection{Pencil beam algorithm}

In 1986, Mohan et al introduced the differential PB model for photon dose computation. Since then others have developed practical means of applying this model for use in radiotherapy clinics (Ahnesjö et al 1992, Gustafsson et al 1994, Ostapiak et al 1997). The PB model is an efficient semi-analytical dose calculation algorithm that was introduced when convolution/superposition models were still too computationally expensive to be used routinely in the clinic. The PB kernel is formed by convolving point dose kernels with elemental (PB) photon fluence distributions (Ostapiak et al 1997). In other words, knowledge of the variation of the fluence with depth permits the convolution of the point dose spread with the x-ray fluence along this direction. This preconvolution increases the efficiency of the dose calculation algorithm by pre-calculating the dose spread in one dimension. At each depth $(z)$, the polyenergetic PB kernel is fitted to the following expression:

$$
\frac{p(\vec{r}, z)}{\rho}=\frac{A_{z} e^{-a_{z} r}}{r}+\frac{B_{z} e^{-b_{z} r}}{r}
$$


where $\frac{p(\vec{r}, z)}{\rho}$ is the energy fraction deposited per unit mass and $r$ is the radial distance. The depth-dependent fitting parameters $A_{z}, a_{z}, B_{z}$ and $b_{z}$ are predetermined and used by the treatment planning system at run time. These PB kernels are then convolved or superpositioned with the primary energy fluence to calculate the three-dimensional dose distribution. Ahnesjö et al (1992) validated this model in the penumbra region and addressed charged particle and photon contamination.

In heterogeneous media, the primary dose is calculated by replacing the geometrical depth with the radiological depth $\left(z_{e f f}\right)$. The scatter dose is calculated by using the fitting parameters at the geometrical depth but with the application of a scattering correction factor $\left(C F_{s}\right)$ where:

$$
C F_{s}=\left(\frac{z_{\text {eff }}}{z}\right) \exp \left[-\bar{\mu}\left(z_{\text {eff }}-z\right)\right]
$$

This means that the scatter dose is first calculated in homogeneous media and is subsequently corrected for a one-dimensional inhomogeneity correction along the beam path. Empirically, it has been found that approximating $\bar{\mu}$ to be $0.8 \mu$ (where $\mu$ is the linear attenuation coefficient of the primary photons) is sufficient for 1-D inhomogeneity correction. It is evident that the main limitation in accuracy for the PB model is the manner in which it handles tissue heterogeneities and scattered dose. Effects such as penumbra widening in the lungs are not calculated accurately since lateral electron transport is neglected. 


\subsection{Lung dose measurements and calculations}

Some of the largest errors in treatment planning dose calculations occur in the vicinity of tissue heterogeneities. In most cases, this is due to an inadequate modeling of the lateral electron transport (Arnfield et al 2000). Lateral electronic disequilibrium exists in areas where the lateral range of the secondary electrons is greater than the distance to the photon field edge. This effect is often evident in the lungs where there is a large lowdensity region (relative to water) surrounded by normal tissue.

Treatment planning corrections for low-density inhomogeneities have been studied since the early 1980s (Beach et al 1987, El-Khatib and Battista 1984, El-Khatib and Battista 1986, Mackie et al 1985). Methods to account for tissue inhomogeneities have continued to develop along with advances in TPS calculation algorithms. This section of the thesis will examine the Oncentra implementation of the PB and CCC algorithms; in particular it will examine the dose calculation accuracy in low-density media. The treatment planning calculations were compared to TLD (thermo-luminescent dosimeter) measurements and verified with studies performed by other researchers.

\subsubsection{Material and Methods}

All measurements were performed on a Siemens Mevatron linear accelerator at The Ottawa Hospital Cancer Centre (TOHCC). Dose measurements were taken with lithium fluoride TLD-100 (Harshaw Chemical Company, Ohio, USA) ribbons placed in a $30 \mathrm{~cm} \times$ $30 \mathrm{~cm} \times 8 \mathrm{~cm}$ acrylic phantom with two rectangular lobes filled with lung-equivalent material as shown in Figure 3.1. Further details on the use of the TLD-100 ribbons are 
discussed in section 3.3.1.2. The lung lobe portions were segmented into 8 slices with one of the eight slices machined to fit a matrix of 48 TLD chips. Figure 3.2 shows the beam geometries investigated in this study, each designed to examine a specific dose distribution in lung-equivalent material. The three experimental geometries, labeled $5 \times 5 i, 5 \times 5 i i$ and $10 \times 10$, are described in detail in section 3.3.2.

For each beam geometry, measurements were made at four depths and three photon beam energies (6MV, 10MV and 18MV). Lucite plates were placed distal and proximal on the surface the phantom to provide adequate buildup (and backscatter) prior to the lung inhomogeneities. Monitor units were calculated to deliver $100 \mathrm{cGy}$ to the beam isocenter at a depth of $8.33 \mathrm{~cm}$ in water (at $x=0 \mathrm{~cm}, y=0 \mathrm{~cm}, \mathrm{z}=0 \mathrm{~cm}$ ) with no inhomogeneities present. The number of monitor units (MU) were calculated as follows:

$$
M U=\frac{D_{i}}{\dot{D}_{i}}
$$

where $\mathrm{D}_{\mathrm{i}}$ is the prescribed dose (100 cGy) from field $\mathrm{i}$ per fraction to point $\mathrm{P}$ and $\dot{D}_{i}$ is the dose rate (cGy/MU) at the point of interest for field $i$. The dose rate is calculated as follows:

$$
\dot{D}_{i}=\dot{D}_{o} \cdot S_{h} \cdot S_{p} \cdot T P R \cdot I S L \cdot F_{w} \cdot F_{T}
$$

where $\dot{D}_{o}$ is the reference dose rate, $S_{h}$ is the head scatter factor, $S_{p}$ is the phantom scatter factor, TPR is the tissue-phantom ratio, $F_{w}$ is the wedge factor and $F_{T}$ is the tray factor. $I S L$ represents the inverse-square law correction that accounts for differences between the reference distance (calibration point) and actual distance to the calculation point. 
The buildup region refers to the dose region between the surface $(\mathrm{z}=0)$ and the depth of dose maximum $\left(\mathrm{z}=\mathrm{Z}_{\max }\right)$ in a depth-dose curve. The existence of the build region results from secondary charged particles (released via photon interactions) traveling a relatively long distance before losing all of their energy within the material. To ensure that the measurements are taken beyond the buildup region, it was necessary to place buildup material with a thickness greater than $z_{\max }$ on top of the phantom surface. The depth of dose maximum depends on beam energy and field size, though the latter dependency is minor. Data for beams at The Ottawa Hospital Regional Cancer Centre show that $z_{\max }$ for the 6 and $18 \mathrm{MV} 10 \times 10 \mathrm{~cm}^{2}$ photon beams are 1.6 and $3.0 \mathrm{~cm}$, respectively. These values can vary depending on the individual linear accelerator and any adjustments made during commissioning. The total buildup and backscatter thickness used in these measurements was $4.35 \mathrm{~cm}$ and $1.90 \mathrm{~cm}$, respectively.

A full CT dataset of the phantom (3mm slice thickness with $3 \mathrm{~mm}$ spacing, $512 \times 512$ matrix resolution) was acquired on a Philips AcQsim large bore $(50 \mathrm{~cm}$ field-ofview), single slice CT simulator (Philips Medical Systems, Markham, ON, Canada) at a nominal beam energy of $120 \mathrm{kV}$. Treatment planning dose calculations (using PB and collapsed-cone convolution algorithms) based on this dataset were performed by Theraplan Plus version 3.8 with Oncentra DCM version 2.0 (MDS Nordion, Ottawa, ON, Canada). The dose calculation grid consisted of points spaced $5 \mathrm{~mm}$ apart in both the $\mathrm{x}$ and $\mathrm{y}$ directions of each slice. The conversion between Hounsfield units to relative electron density was based on the data given in Table 3.1 and the curves shown in Figure 5.6. This data was obtained by performing $120 \mathrm{kVp} \mathrm{CT}$ scans of a Gammex RMI 
electron density CT phantom (RMI 467, serial \# 802428-1035, Gammex RMI, Middleton, WI, USA) that includes materials of known electron density. The Philips AcQsim scanner has an inherent limit on the HU, reached between the density of aluminum and steel. This is evident in Table 3.1 where all materials denser than aluminum have reached the HU limit just below 3000. 
Table 3.1 Relative electron density (RED) and Hounsfield unit (HU) data for the Philips AcQsim large bore single slice CT simulator (Philips Medical Systems, Markham, ON, Canada).

\begin{tabular}{ccc}
\hline Material & RED & HU \\
\hline air & 0.0012 & -1000 \\
lung (LN-300) & 0.28 & -689.3 \\
lung (LN-450) & 0.40 & -550.8 \\
adipose & 0.90 & -92.0 \\
breast & 0.96 & -56.6 \\
water & 1.00 & 0.0 \\
brain & 1.05 & 13.6 \\
liver & 1.07 & 82.3 \\
inner bone & 1.09 & 225.6 \\
bone B-200 & 1.11 & 222.4 \\
CB2-30\% & 1.28 & 470.7 \\
CB2-50\% & 1.47 & 832.7 \\
$\begin{array}{c}\text { cortical bone } \\
\text { aluminum } \\
\text { stainless } \\
\text { steel } \\
\text { brass }\end{array}$ & 1.69 & 1271.8 \\
copper & 3.12 & 1604.7 \\
lead & 6.56 & 2977.0 \\
\hline & 10.78 & 2979.3 \\
\hline
\end{tabular}


8 lung-equivalent plates in each lobe Slice dimensions: $8.0 \times 13.0 \times 1.0 \mathrm{~cm}^{3}$

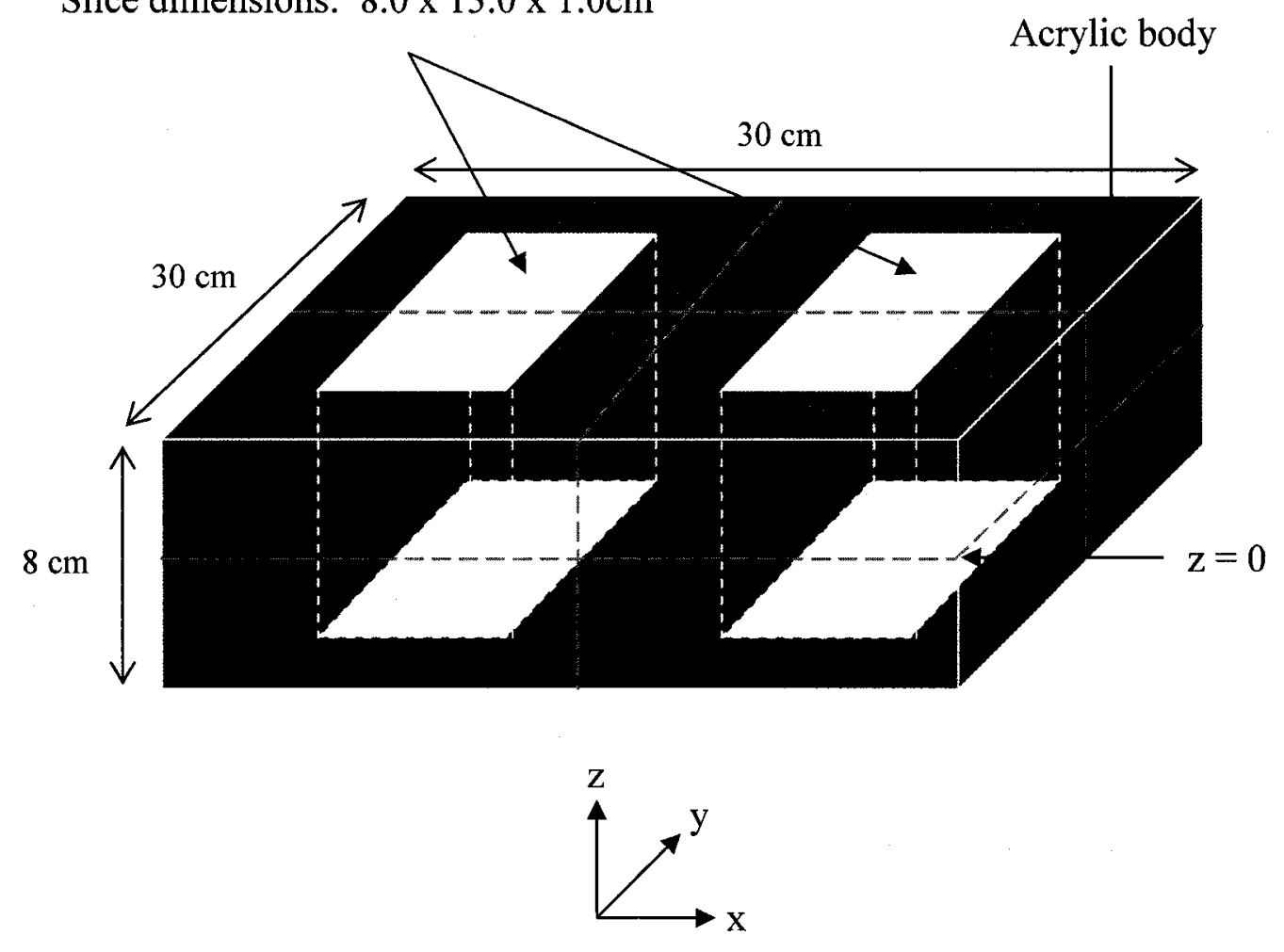

Figure 3.1 Diagram of the acrylic phantom containing two lung equivalent inhomogeneities. Each lung lobe consists of 8 slices. Each slice is machined to accommodate TLD 100 chips, to permit TLD dose measurements at 9 depths throughout the lung-equivalent volume. 


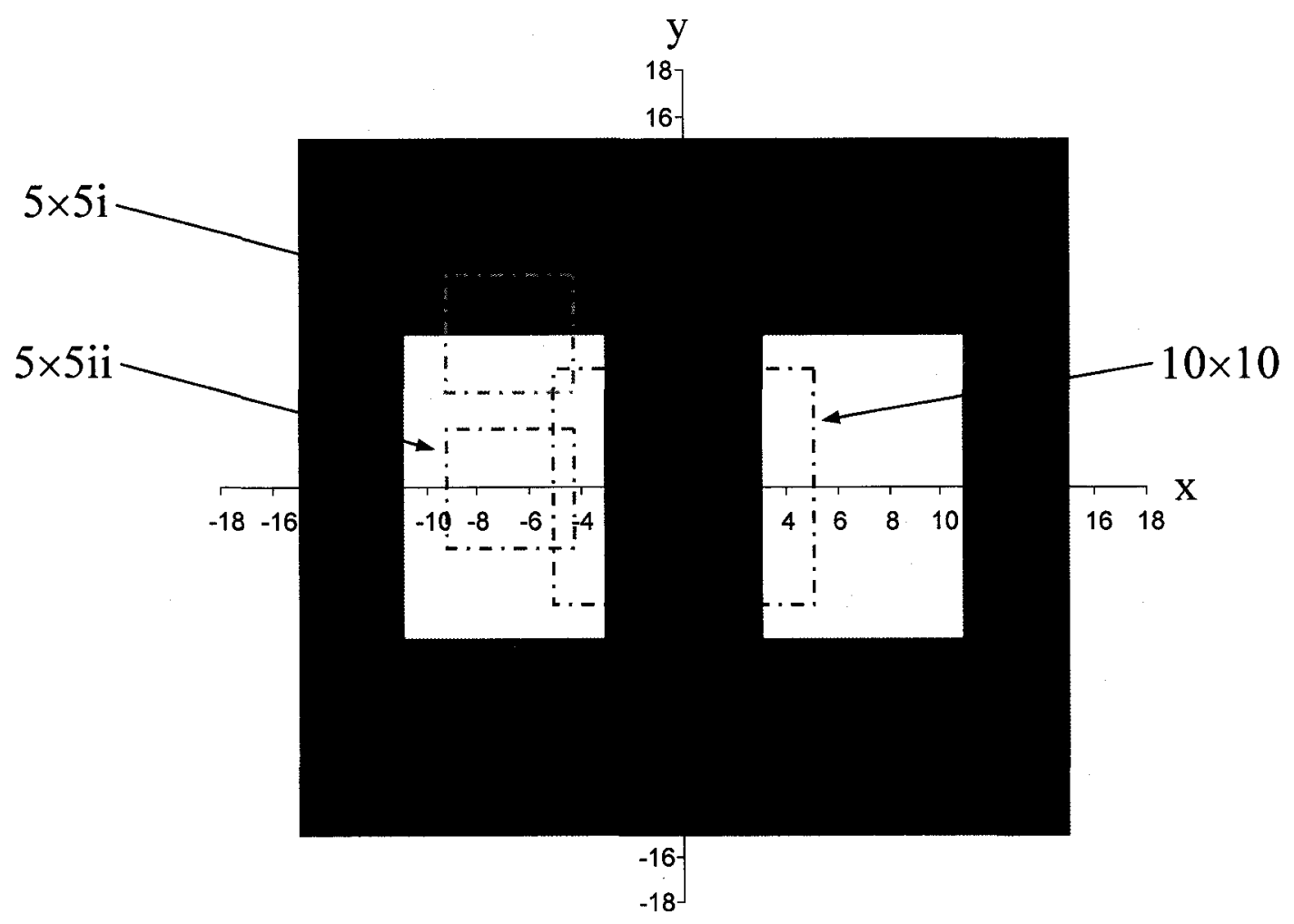

Figure 3.2 Beams eye view of a plane through the middle of the phantom $(\mathrm{z}=0 \mathrm{~cm}$, see Figure 3.1). The dashed squares represent the field edges of three beam geometries (labeled $\mathbf{5} \times \mathbf{5 i}, \mathbf{5} \times \mathbf{5 i i}$ and $\mathbf{1 0} \times \mathbf{1 0}$ ) used in this work. Measurement positions are defined using the coordinates shown. 


\subsubsection{Measurement and Calculation Error}

Since the goal of this study is to evaluate the Theraplan Plus implementation of two dose calculation algorithms, it is important to distinguish between measured and calculated error. For these experiments, the main sources of measurement error are geometric error and TLD error. Errors related to the geometry predominantly include most setup errors such as beam placement, the accuracy of the field size settings and phantom positioning. The clinical tolerance for the field size is $\pm 2 \mathrm{~mm}$ (as advised by the CAPCA Quality Standards Control for Medical Linear Accelerators, 2005) and is measured daily. Additionally, the tolerance for the positional laser systems is $\pm 1 \mathrm{~mm}$, checked daily with quality assurance (QA) testing. This means that we can assume the phantom setup perpendicular to the beam axis is within this uncertainty. The field size and phantom setup errors can be combined to give an estimate of the geometric error. The uncertainty parallel to the beam axis is also $\pm 1 \mathrm{~mm}$, which translates to approximately $0.2 \%$ dose error. The other main source of error arises from TLD error, representing the reproducibility of the TLD measurements. The characteristics of the TLDs will be discussed in the next section. In regions of high dose gradients, the measurements are much more sensitive to geometric error than TLD error. Conversely, the TLD error tends to dominate in low dose gradient regions. For these reasons, it is standard practice to measure the distance to agreement for high dose gradient regions and the dose error in low gradient regions. This study will report dose errors in low dose gradient regions, as will be shown in section 3.3.2. Calculation errors include approximations and limitations of the dose calculation algorithms and TPS and will be examined in section 3.3.3 (Discussion). 


\subsubsection{Thermo-luminescent Dosimeters (TLDs)}

When TLD materials (crystals) are irradiated with ionizing radiation, free electrons are excited into higher energy states and are trapped in vacancies in the crystal lattice structure. The vacancies are due to impurities (such as magnesium and titanium) present in the crystal. Heating the crystal after irradiation causes the lattice to vibrate and release the trapped electrons back into their original ground states. The energy released in this transition is emitted as visible electromagnetic radiation that can be detected by a photomultiplier tube. The number of photons emitted is directly proportional to the amount of energy deposited in the crystal structure.

The TLD-100 ribbons have dimensions of $3.2 \mathrm{~mm} \times 3.2 \mathrm{~mm} \times 0.9 \mathrm{~mm}$. The ribbons are composed of lithium fluoride crystals doped with magnesium and titanium with a physical density of $2.64 \mathrm{~g} / \mathrm{cm}^{3}$. They are sensitive to photons $(>5 \mathrm{keV})$, neutrons (thermal to $100 \mathrm{MeV}$ ) and electrons $(>70 \mathrm{keV})$ with a linear dose response range between $10 \mu \mathrm{Gy}$ to $1 \mathrm{~Gy}$. The small dimensions and near tissue equivalence (atomic number) allow for relatively high resolution measurements in phantoms with minimal perturbation in the phantom material. Other benefits of using the TLD-100 ribbons are their reusability, reproducibility (within $2 \%$ ), long term response retention, and independence to dose rate up to $100 \mathrm{MGy} / \mathrm{s}$.

Manufacturer specifications (Harshaw Chemical Company, Ohio, USA) state approximate tissue equivalence of lithium fluoride crystals $\left(Z_{\text {eff }}=8.14\right)$ compared to effective atomic numbers for soft tissue $\left(Z_{\text {eff }}=7.42\right)$. However, it should be stressed that 
this comparison is only approximate since it has been shown that $Z_{\text {eff }}$ is energy dependent (Prasad et al 1997). This difference in effective atomic numbers can result in systematic errors when performing dose measurements with LiF TLD chips. In this study, dose measurements are performed in lung-equivalent material. Although the TLD-100 chips are relatively small, there are small perturbations of the lung-equivalent material. The TLD chips were calibrated in a water-equivalent acrylic phantom and irradiated to 100 cGy with a $10 \times 10 \mathrm{~cm}^{2}$ field of Co60 photons. The Co60 treatment unit was calibrated to provide dose to water within $\pm 1 \%$ uncertainty. Therefore, the TLD chips were calibrated to measure dose to water. As mentioned in section 3.2, the PB and $\mathrm{CCC}$ algorithms are based on dose deposition kernels measured in water as opposed to tissue. This approximation has been shown to be acceptable over the range of densities found in various human tissues.

For accurate TLD measurements that account for the differences in detector and phantom materials, the following procedure can be applied (Heath, 2003). To convert the dose to the detector $\left(D_{d e t}\right)$ to the dose the medium $\left(D_{m e d}\right)$, Spencer-Attix cavity theory states that:

$$
D_{m e d}=D_{\operatorname{det}}\left[\left(\frac{\bar{L}}{\rho}\right)_{\mathrm{det}}^{\text {med }}\right] \bar{\Phi}_{\mathrm{det}}^{\text {med }}
$$

where $\left(\frac{\bar{L}}{\rho}\right)_{\mathrm{det}}^{\text {med }}$ is the restricted stopping power ratio and $\bar{\Phi}_{\mathrm{det}}^{\text {med }}$ is a correction factor for the fluence perturbation produced by the detector. The dose deposited in the TLD, $D_{t l d}$, can be calculated as follows:

$$
D_{t l d}=M_{t d} N_{t l d}
$$


where $M_{t l d}$ is the TLD reading and $N_{t l d}$ is the calibration factor. The TLD reading can then be related to the dose in the calibration phantom medium (e.g. acrylic), represented by $D_{a c r y l}(c a l)$, by combining equations 3.20 and 3.21 :

$$
D_{a c r y l}(c a l)=M_{t l d}(c a l) N_{t l d}\left[\left(\frac{\bar{L}}{\rho}\right)_{\mathrm{det}}^{\text {med }} \bar{\Phi}_{\mathrm{det}}^{\text {med }}\right]
$$

The calibration dose in water, $D_{w}^{Q}(c a l)$, is determined from ion chamber measurements as recommended by the TG-51 protocol:

$$
D_{w}^{Q}(c a l)=M_{c o r r} N_{D, w}^{C o 60} k_{Q}
$$

where $M_{\text {corr }}$ is the ion chamber measurement corrected for temperature and pressure, collection efficiency and polarity, $N_{D, w}^{C o 60}$ is the calibration factor for the dose to water in a ${ }^{60} \mathrm{Co}$ beam and $k_{Q}$ is the quality conversion factor. The calibration dose in water, $D_{w}(\mathrm{cal})$, can then be used to determine the calibration dose in acrylic:

$$
D_{a c r y l}(c a l)=\left[\left(\frac{\bar{\mu}_{a b}}{\rho}\right)_{w}^{a c r y l} \beta_{w}^{a c r y l}\right] D_{w}(c a l)
$$

where $\left(\frac{\bar{\mu}_{a b}}{\rho}\right)_{w}^{a c r y l}$ is the ratio of mass energy absorption coefficients of acrylic over water. By equating equations (3.22) and (3.24), $N_{t l d}$ can be determined and consequently, equations (3.20) and (3.21) can be used to solve for the dose to the medium. In Heath's work, Monte Carlo techniques were used to obtain values for the stopping power ratios, mass energy absorption coefficients and the dose perturbation correction factor. However, this level of accuracy was beyond the scope of our work and was not considered in this study. 
The preparatory annealing procedures were outlined by the TLD manufacturer (Harshaw Chemical Company, Ohio, USA) and have been shown to produce accurate results (Essers and Mijnheer 1999, Yu and Luxton 1999). Prior to irradiation, all TLDs were pre-annealed at $400^{\circ} \mathrm{C}$ for 1 hour in a Thermolyne 10500 furnace (BarnsteadThermolyne Corporation, Dubuque, IN, USA) followed by 20 minutes of cooling at room temperature. This was immediately followed with heating at $100^{\circ} \mathrm{C}$ for 2 hours in a Thermolyne 19200 heater (Barnstead-Thermolyne Corporation, Dubuque, IN, USA) and cooling to room temperature once again. This annealing process drops all electrons to their ground states and resets the TLDs for re-use. For consistency, all irradiation experiments were performed at approximately the same time after the pre-annealing stage. Similarly, all post-irradiation annealing procedures were performed approximately the same amount of time after irradiation and prior to reading. The pre-reading annealing procedure consisted of heating the TLDs at $100^{\circ} \mathrm{C}$ for 10 minutes. The ribbons were subsequently read with a Harshaw QS 5500 TLD reader (Harshaw Chemical Company, Ohio, USA).

The reproducibility of TLDs has been shown to be about $2 \%$ for one standard deviation (Essers and Mijnheer 1999, Kron 1995), though this is dependent upon the handling and calibration of the dosimeters. For the measurements, the TLDs were individually calibrated on a regular basis both prior to and after the experiments. The reproducibility of the TLD chips was examined by analyzing data from three consecutive calibration experiments taken within a short span of time (6 days) as shown in Table A.1 in Appendix A. In each calibration experiment, the TLD chips were placed in a 
calibration phantom with sufficient build-up and backscatter plates and irradiated to 100 cGy with $\mathrm{Co}^{60}$ photons. This TLD calibration is dependent upon the $\pm 1 \%$ uncertainty of $\mathrm{N}_{D, w}$ (NRC calibration) which is used to calibrate the $\mathrm{Co}^{60}$ treatment unit. Including field size and phantom setup errors, the estimated uncertainty on the TLD calibration is about $2 \%$. The relative dose response is different for each TLD chip and is dependent upon the irradiation and thermal history. Tables A.1 and A.2 (Appendix A) show that the response of all of the TLD chips used in our experiments could be reproduced with one standard deviation of less than $2 \%$. Combining the calibration and reproducibility errors gives an estimate of $\pm 2.8 \%$ uncertainty on the TLD measurements.

\subsubsection{Treatment planning system lung dose errors}

Measured (TLD) and calculated (TPS) dose profiles as well as percentage difference curves for the $5 \times 5 \mathrm{~cm}^{2}$ and $10 \times 10 \mathrm{~cm}^{2}$ beam geometries (shown in Figure 3.2) for $6 \mathrm{MV}$, $10 \mathrm{MV}$ and $18 \mathrm{MV}$ photon energies are shown in Figures 3.3 through 3.20. The dose difference curves show the relative dose difference between TPS calculations and measurements for each experiment. The relative percentage dose difference was calculated as follows:

$$
\text { relativeDoseDifference }=\left(\frac{\text { Calculated }- \text { Measured }}{\text { Measured }}\right) \times 100 \%
$$

For brevity, this chapter will refer to the beam geometries illustrated in Figure 3.2 as the $5 \times 5 i, 5 \times 5 i i$ and $10 \times 10$. The $5 \times 5 i$ geometry is such that the center of the beam is coincident with the lung-acrylic interface. The superior half of the beam is incident upon acrylic and the inferior half is incident upon the lung-equivalent material. The $10 \times 10$ 
geometry is such that the center of the beam is coincident on the central axis of the phantom. The majority of the field lies within the acrylic material but the lateral edges lie within the lung-equivalent material. The $5 \times 5 i$ and $10 \times 10$ geometries were selected to mimic lung-mediastinum interfaces and to investigate TPS dose calculations in this region. The $5 \times 5 i i$ geometry is such that the entire beam lies within the lung-equivalent material.

$\mathrm{PB}$ and $\mathrm{CCC}$ calculations are shown for all energies. Each figure shows the dose profile at four depths in the lung-equivalent portion of the phantom. The profiles are labeled according to their depth in the lung-equivalent material (depth does not include the acrylic buildup material lying above the lung-equivalent material). The depths are defined at the top surface of the TLD chips, which have a thickness of approximately 1 $\mathrm{mm}$. The dose profile at a depth of $0.0 \mathrm{~cm}(\mathrm{z}=4.0 \mathrm{~cm})$ represents the lung-acrylic interface, measured at the top surface of the lung phantom and distal to the buildup material. Subsequently, a depth of $1.0 \mathrm{~cm}(\mathrm{z}=3.0 \mathrm{~cm})$ shows the dose profile one centimeter below the top interface. A profile midway through the lung-equivalent material is shown at a depth of $3.9 \mathrm{~cm}(\mathrm{z}=0.1 \mathrm{~cm})$. The fourth depth examined was located at the exit lung-acrylic interface at a depth of $7.9 \mathrm{~cm}(\mathrm{z}=-3.9 \mathrm{~cm})$, measured at the bottom surface of the lung-equivalent material and immediately above the backscatter material. Profiles for the $5 \times 5 i$ and $5 \times 5 i i$ beam geometries are shown along $\mathrm{x}=-7.5 \mathrm{~cm}$, and for the $10 \times 10$ beam geometry along $\mathrm{y}=3.0 \mathrm{~cm}$ (Figure 3.2). 


\section{A. $5 \times 5 i$ beam geometry}

In this geometry, half the beam traverses tissue-like material and half the beam traverses lung-like material. Figures 3.3 to 3.8 show the dose profiles and dose difference curves for the $5 \times 5 i$ beam geometry at all photon beam energies, respectively. The dotted line represents the geometric beam edge. All data points to the right of this line lie within the geometric photon field. The dose profiles are analyzed in two main regions, the penumbra and plateau regions. The penumbra defines the region from $20 \%$ to $80 \%$ of the maximum dose. The upper-penumbra refers to the region between $50 \%$ and $80 \%$ of maximum dose and the lower-penumbra refers to the region between $20 \%$ and $50 \%$ of maximum dose. The plateau region dose refers to the dose points near the central axis of the geometric beam where the dose gradient would be expected to be small. The experimental reproducibility of the TLD measurements was estimated by repeating a single experiment three times and determining the standard deviation of each dose point.

For the $6 \mathrm{MV}$ beam, discrepancies between measured and calculated dose were observed (Figure 3.3 and 3.4). At a distance of $2 \mathrm{~cm}$ and greater outside the geometric beam, both the PB and CCC algorithms agree well with measurement. At approximately $1 \mathrm{~cm}$ outside the geometric beam edge, the PB algorithm underestimates the dose (up to 4.9\%) but the $\mathrm{CCC}$ algorithm estimates the dose quite well. At the geometric beam edge, we see the largest dose difference. At about $1 \mathrm{~cm}$ inside the beam edge, you see that the error in the PB calculation increases (up to 5.5\%) with depth while the CCC results are fairly good and relatively depth independent. Finally in the plateau region, the PB algorithm seems to perform better than the CCC algorithm. 
For the $10 \mathrm{MV}$ beam, the dose profiles and percentage dose differences are presented in Figures 3.5 and 3.6, respectively. At a distance of $2 \mathrm{~cm}$ and greater outside the geometric beam, both algorithms again generally agree with measurement. Approximately $1 \mathrm{~cm}$ outside the beam edge, the PB algorithm continues to underestimate the dose (up to $8 \%$ ) but the CCC algorithm still performs quite well. As before, the largest dose differences were observed at the geometric beam edge. At about $1 \mathrm{~cm}$ inside the beam edge, the PB algorithm overestimated the dose, showing the greatest increase (up to $13 \%$ ) at a depth of $1 \mathrm{~cm}$ into the lung-equivalent material. The $\mathrm{CCC}$ algorithm also overestimated the dose (up to $8 \%$ ) at the shallower depths but agreed with measurement deeper into the lung-equivalent material. In the plateau region, the PB and CCC algorithms showed dose errors up to $6 \%$ and $5 \%$, respectively.

For the $18 \mathrm{MV}$ beam, the dose profiles and percentage dose differences are shown in Figures 3.7 and 3.8, respectively. At the geometric beam edge and outside of it, the general behaviour was similar to the $10 \mathrm{MV}$ dose profiles. Approximately $1 \mathrm{~cm}$ inside the beam edge, the PB and CCC algorithms both overestimated the dose at all depths, though showing less dose differences near the distal interface. In the plateau region, both algorithms showed approximately the same overestimation of dose relative to measurement. 

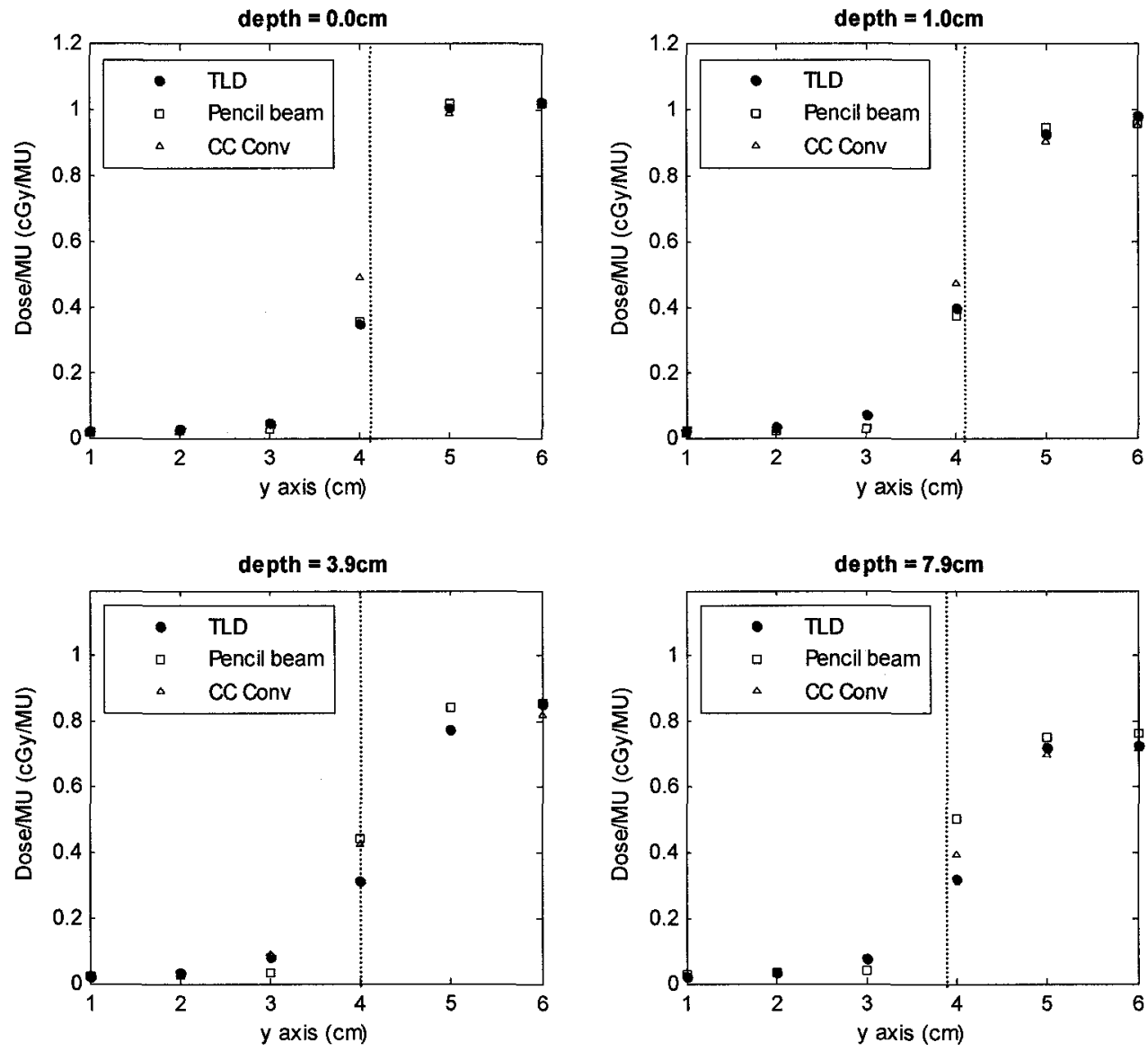

Figure 3.3 Measured (TLD) and calculated (PB and CCC) dose profiles in lung equivalent material for a $6 \mathrm{MV}$ photon beam in the $5 \times 5 i$ beam geometry at four depths below the proximal lung-acrylic interface (depths $=0.0 \mathrm{~cm}, 1.0 \mathrm{~cm}, 3.9 \mathrm{~cm}$ and $7.9 \mathrm{~cm}$ ). All points to the right of the dotted line lie within the primary (geometric) beam. The estimated TLD error is within the size of the symbols. 

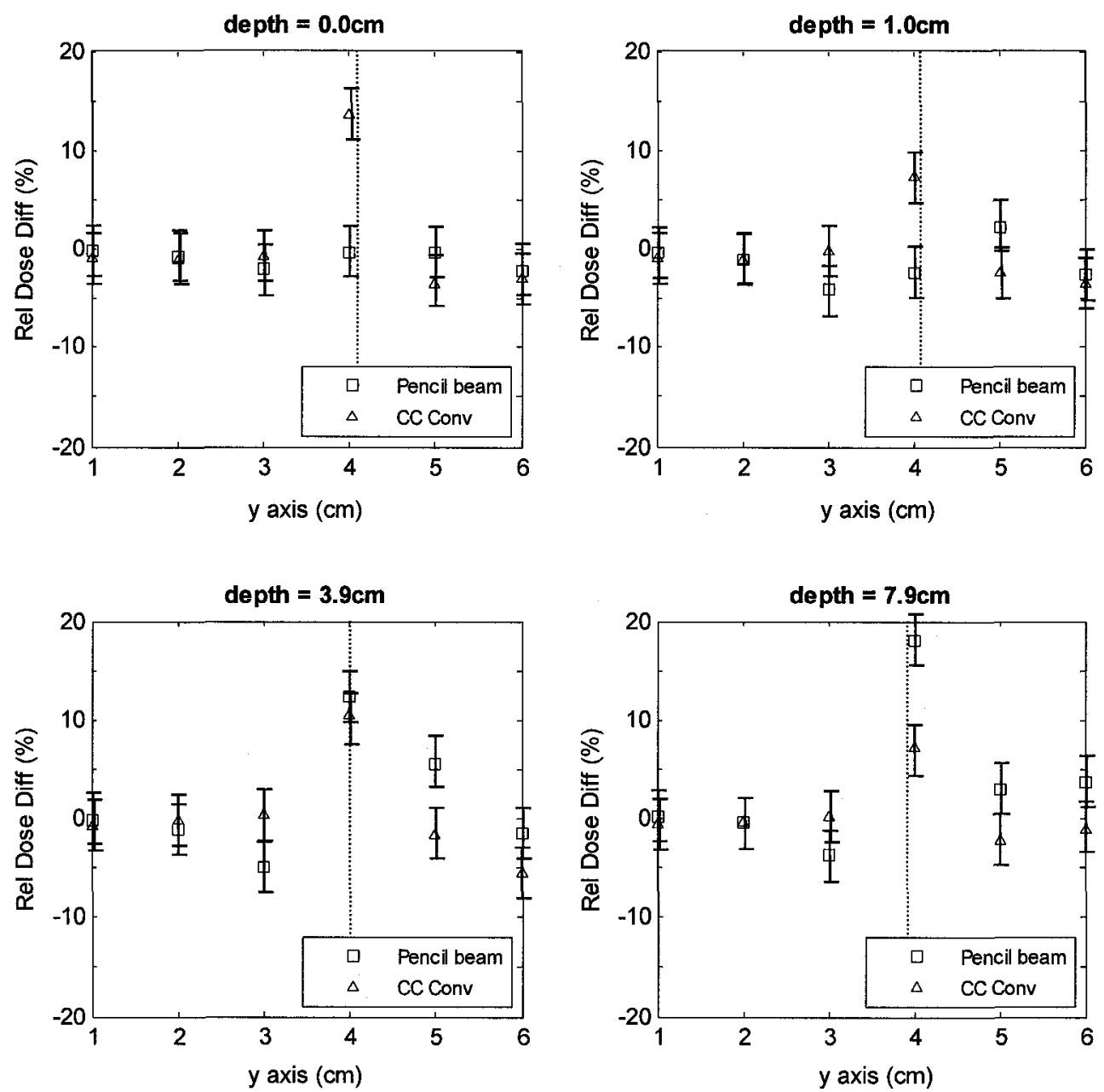

Figure 3.4 The \% dose difference between the calculated (PB and CCC) and TLD measurements for a $6 \mathrm{MV}$ photon beam in the $5 \times 5 i$ experimental geometry at four depths below the proximal lung-acrylic interface. All points to the right of the dotted line lie within the primary (geometric) beam. 

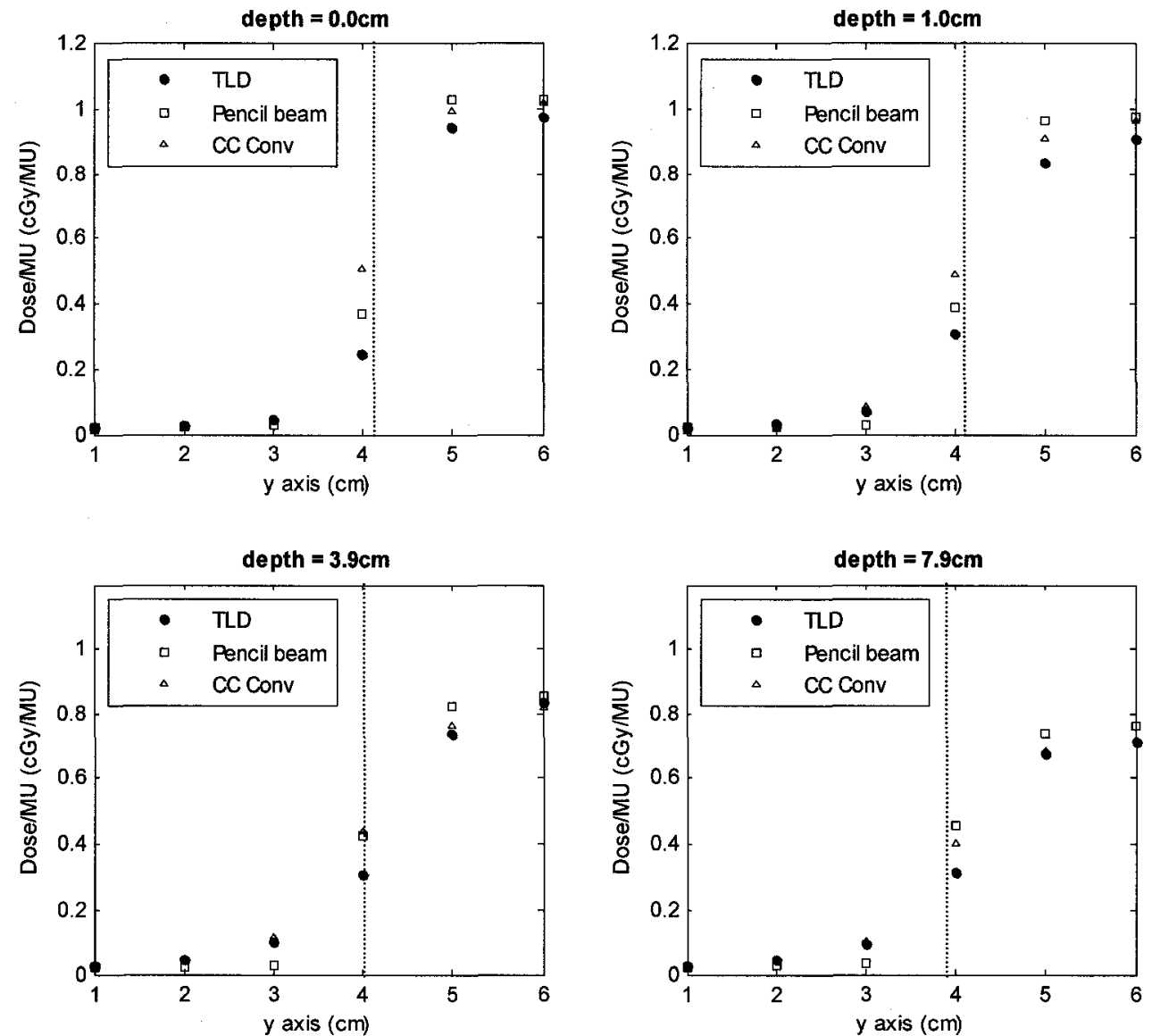

Figure 3.5 Measured (TLD) and calculated (PB and CCC) dose profiles in lung equivalent material for a $10 \mathrm{MV}$ photon beam in the $5 \times 5 i$ beam geometry at four depths below the proximal lung-acrylic interface (depths $=0.0 \mathrm{~cm}, 1.0 \mathrm{~cm}, 3.9 \mathrm{~cm}$ and $7.9 \mathrm{~cm}$ ). All points to the right of the dotted line lie within the primary (geometric) beam. The estimated TLD error is within the size of the symbols. 

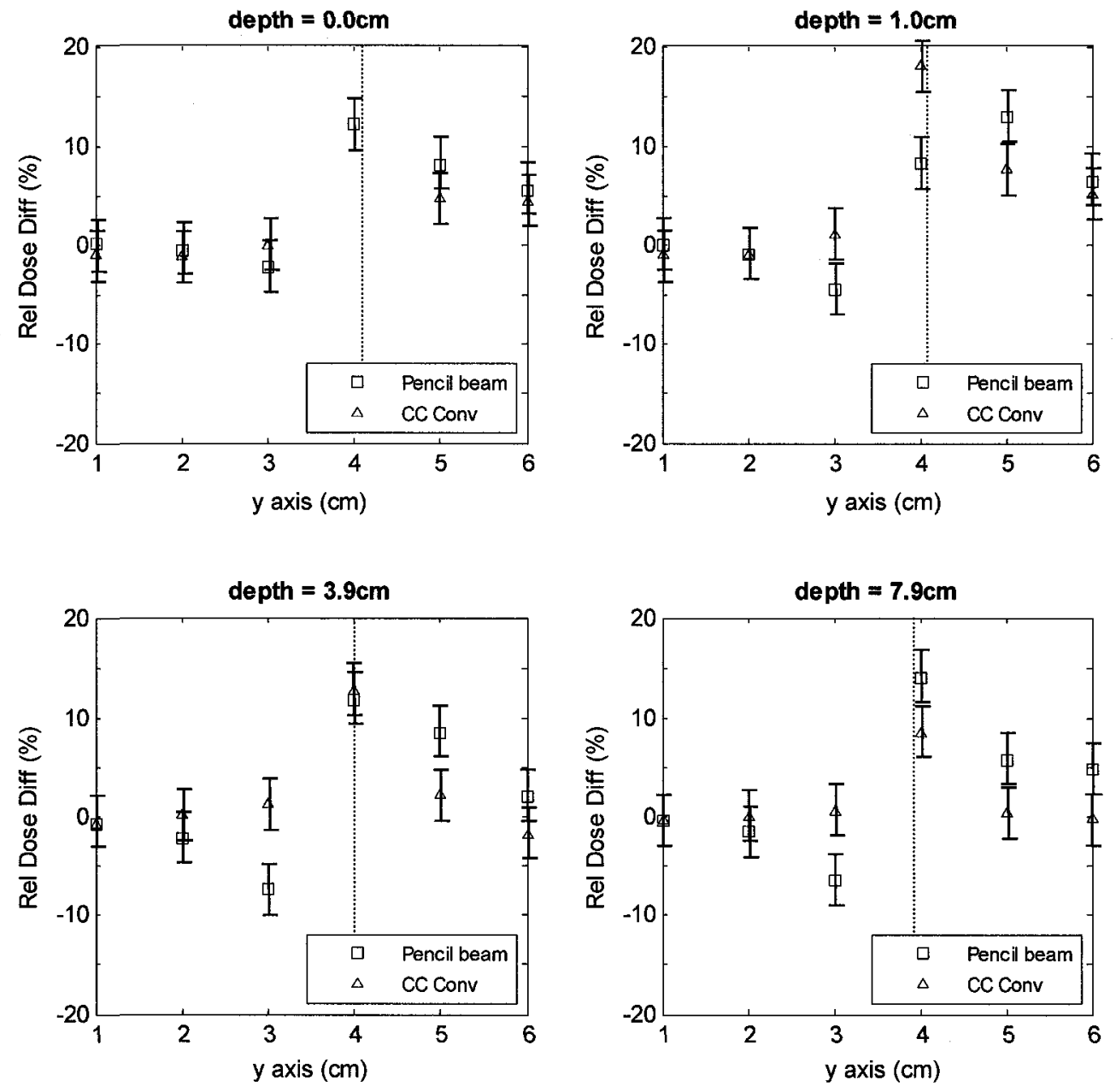

Figure 3.6 The \% dose difference between the calculated (PB and CCC) and TLD measurements for a $10 \mathrm{MV}$ photon beam in the $5 \times 5 i$ experimental geometry at four depths below the proximal lung-acrylic interface. All points to the right of the dotted line lie within the primary (geometric) beam. 

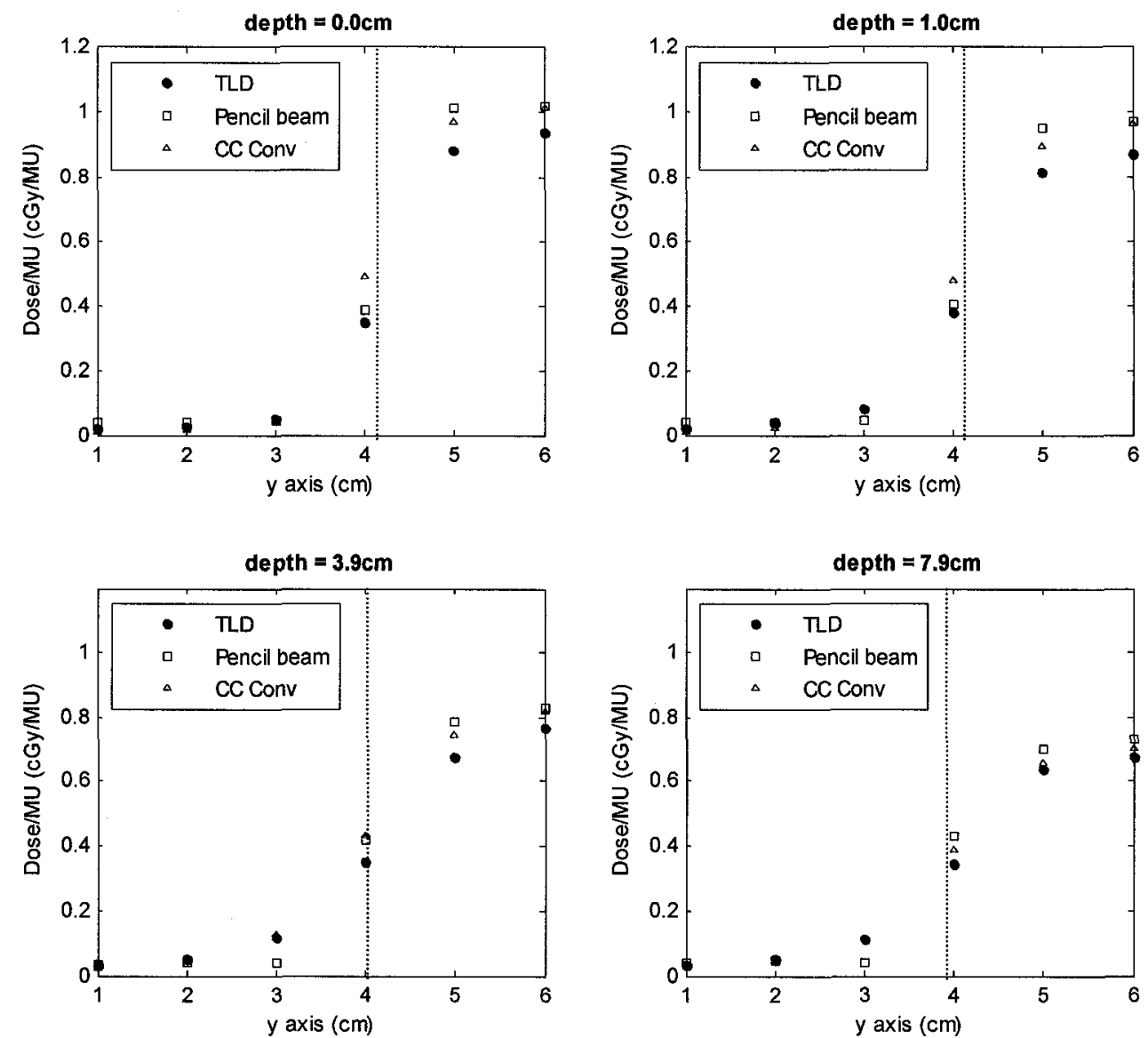

Figure 3.7 Measured (TLD) and calculated (PB and $\mathrm{CCC}$ ) dose profiles in lung equivalent material for a $18 \mathrm{MV}$ photon beam in the $5 \times 5 i$ beam geometry at four depths below the proximal lung-acrylic interface (depths $=0.0 \mathrm{~cm}, 1.0 \mathrm{~cm}, 3.9 \mathrm{~cm}$ and $7.9 \mathrm{~cm}$ ). All points to the right of the dotted line lie within the primary (geometric) beam. The estimated TLD error is within the size of the symbols. 

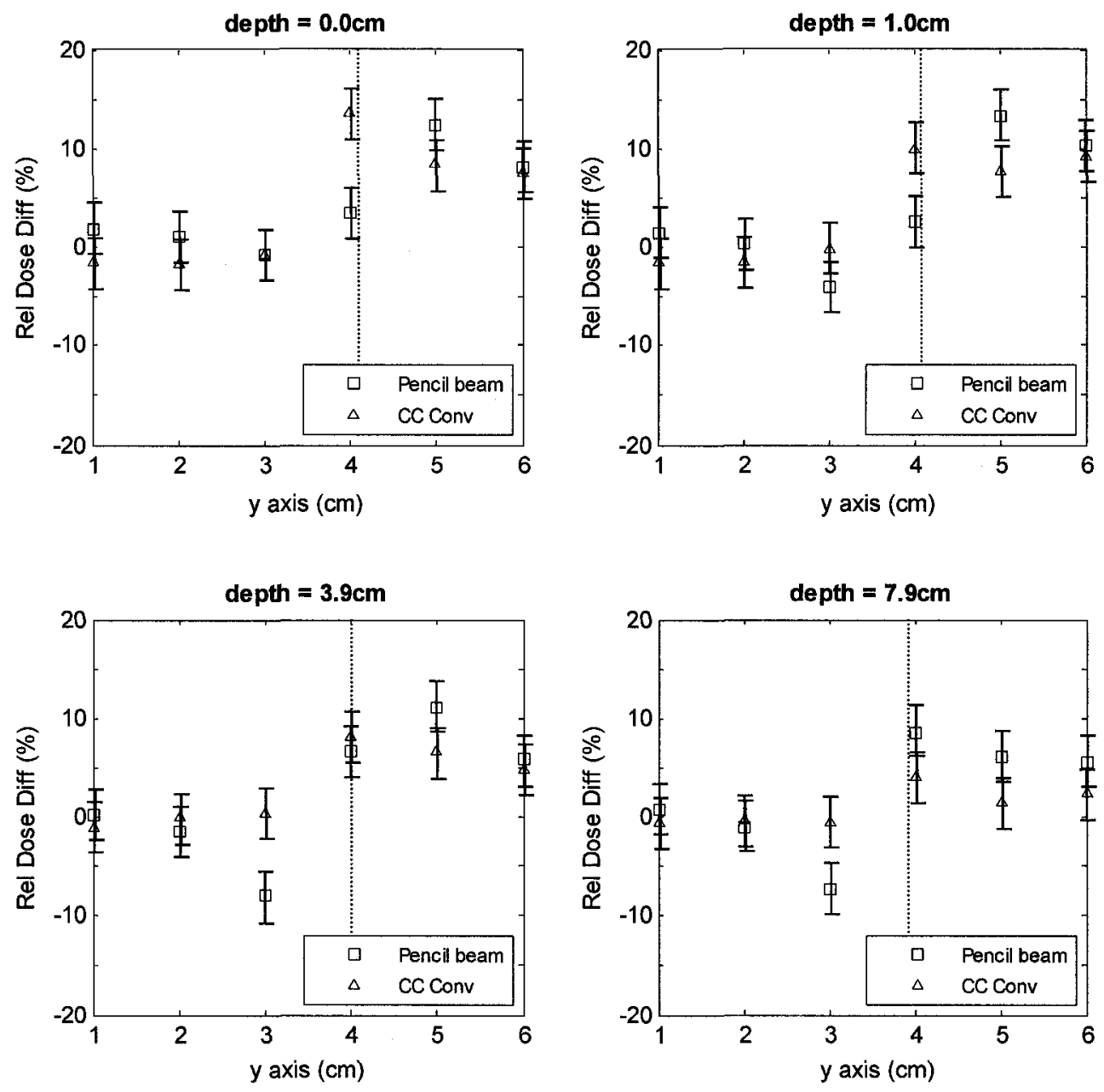

Figure 3.8 The \% dose difference between the calculated (PB and CCC) and TLD measurements for a $18 \mathrm{MV}$ photon beam in the $5 \times 5 i$ experimental geometry at four depths below the proximal lung-acrylic interface. All points to the right of the dotted line lie within the primary (geometric) beam. 


\section{B. $5 \times 5 i i$ beam geometry}

In the $5 \times 5 i i$ geometry, the entire beam transits through lung-equivalent material. The dose profiles and difference curves for the $5 \times 5 i i$ beam geometry are shown in Figures 3.9 to 3.14. For this experimental geometry, all data points left of the dotted line (geometric beam edge) lie within the primary beam. For the $6 \mathrm{MV}$ beam (Figures 3.9 and 3.10), the $\mathrm{PB}$ and $\mathrm{CCC}$ algorithms performed well at a distance of $1.5 \mathrm{~cm}$ and $2.5 \mathrm{~cm}$ outside the primary beam. Approximately $0.5 \mathrm{~cm}$ outside the beam, the PB calculation underpredicted the dose (up to 7\%) and the CCC calculation over-predicted the dose (up to $8 \%$ ). At $0.5 \mathrm{~cm}$ inside the beam, the $\mathrm{PB}$ algorithm overestimated the dose showing, with the greatest dose difference (up to $14 \%$ ) seen $1 \mathrm{~cm}$ below the proximal lung-acrylic interface. The CCC algorithm performed better at this point except at a depth mid-way through the lung material. $1.5 \mathrm{~cm}$ and $2.5 \mathrm{~cm}$ (plateau region) inside the beam, the CCC algorithm again outperformed the PB algorithm, both showing an increase in \% dose difference with increasing depth.

For the $10 \mathrm{MV}$ beam, the dose profiles and difference curves are presented in Figures 3.11 and $3.12 .2 .5 \mathrm{~cm}$ outside the geometric beam edge, both the $\mathrm{PB}$ and $\mathrm{CCC}$ algorithms agreed with measurement. $1.5 \mathrm{~cm}$ outside the beam, the $\mathrm{CCC}$ algorithm continued to agree with the measured data but the PB algorithm under-predicted the dose by up to $6 \%$. At approximately $0.5 \mathrm{~cm}$ outside the primary beam, the PB algorithm drastically underestimated the dose (up to 20\%). The CCC algorithm performed much better at this same point, underestimating the dose by up to $7 \%$. Near the plateau region, 
the CCC algorithm once again performed better than the PB algorithm, although both algorithms tended to over-predict the dose.

Figures 3.13 and 3.14 show the dose profiles and difference curves for the $18 \mathrm{MV}$ beam. At a distance of $1.5 \mathrm{~cm}$ and $2.5 \mathrm{~cm}$ outside the beam edge, the CCC algorithm agreed with measurement. The PB algorithm agreed with measurement at $2.5 \mathrm{~cm}$ but increasingly underestimated the dose with increasing depth at $1.5 \mathrm{~cm}$. At $0.5 \mathrm{~cm}$ outside the beam, the CCC algorithm consistently over-predicted the dose by up around $5 \%$. At the same point, the PB algorithm continued to underestimate the dose (up to $10 \%$ ). Inside the primary beam, the $\mathrm{PB}$ and $\mathrm{CCC}$ algorithms generally over-predicted the dose by up to $22 \%$ and $15 \%$, respectively. 

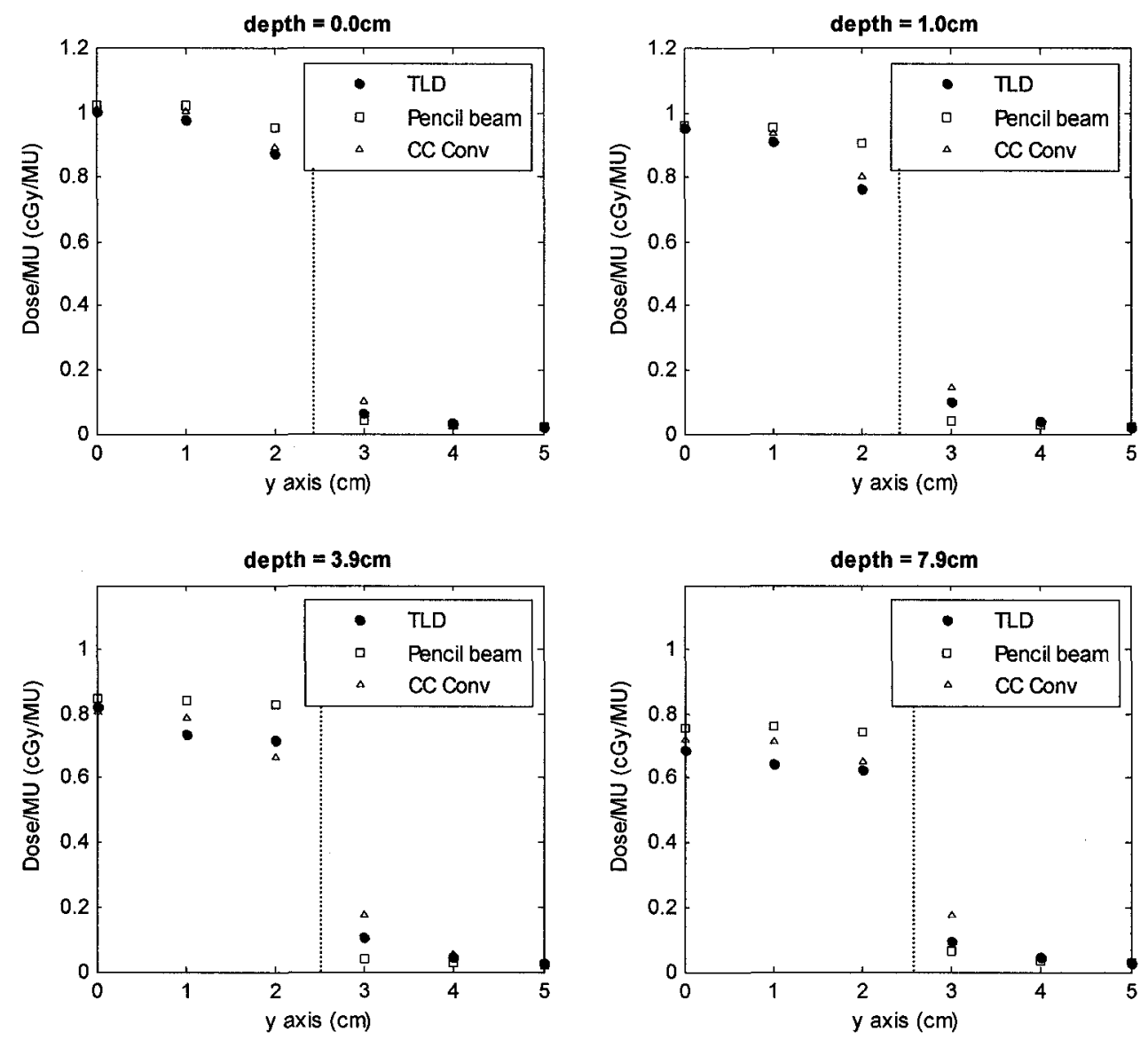

Figure 3.9 Measured (TLD) and calculated (PB and CCC) dose profiles in lung equivalent material for a $6 \mathrm{MV}$ photon beam in the $5 \times 5 i i$ beam geometry at four depths below the proximal lung-acrylic interface (depths $=0.0 \mathrm{~cm}, 1.0 \mathrm{~cm}, 3.9 \mathrm{~cm}$ and $7.9 \mathrm{~cm}$ ). All points to the right of the dotted line lie within the primary (geometric) beam. The estimated TLD error is within the size of the symbols. 

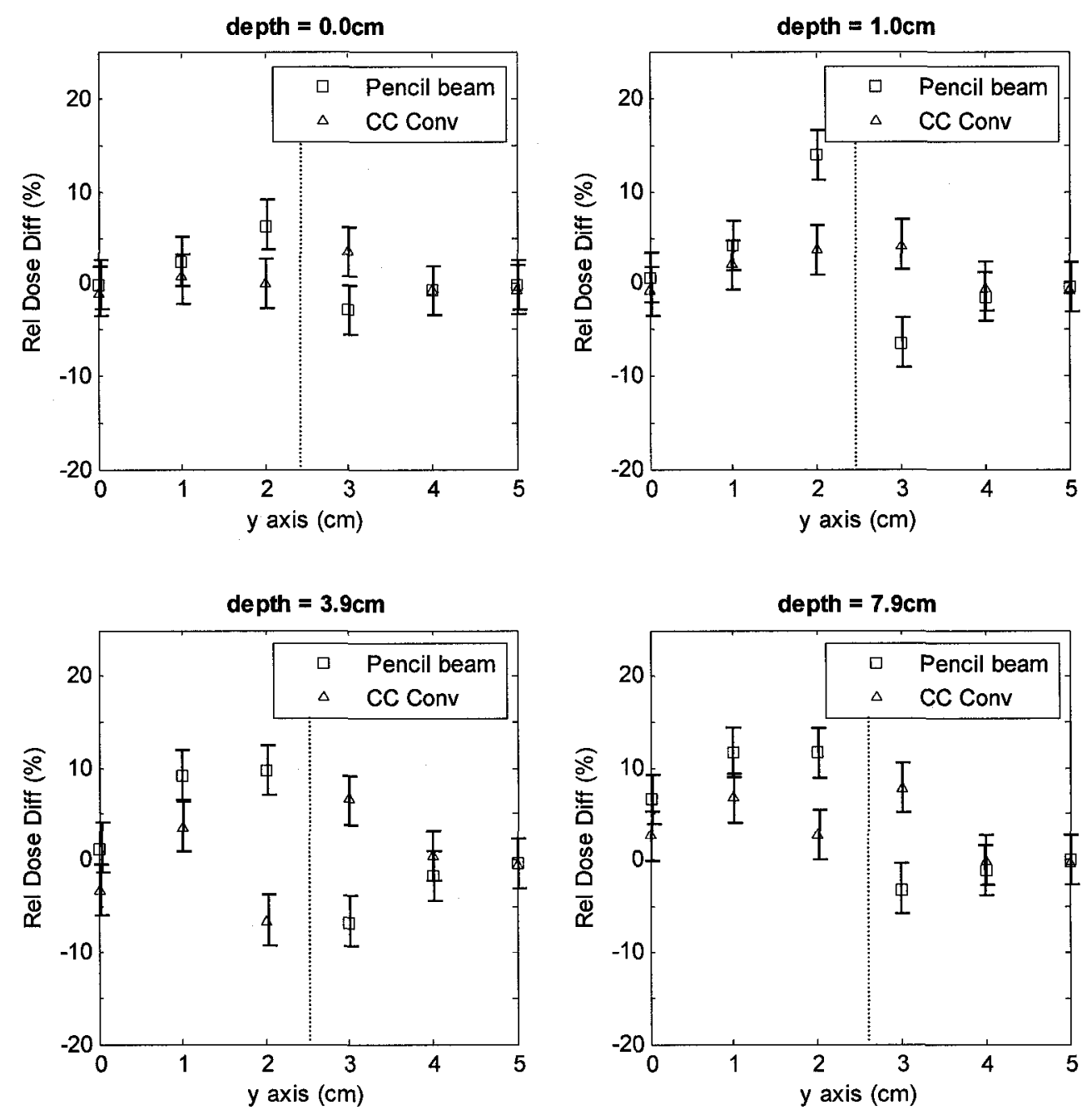

Figure 3.10 The \% dose difference between the calculated (PB and CCC) and TLD measurements for a $6 \mathrm{MV}$ photon beam in the $5 \times 5 i i$ experimental geometry at four depths below the proximal lung-acrylic interface. All points to the right of the dotted line lie within the primary (geometric) beam. 

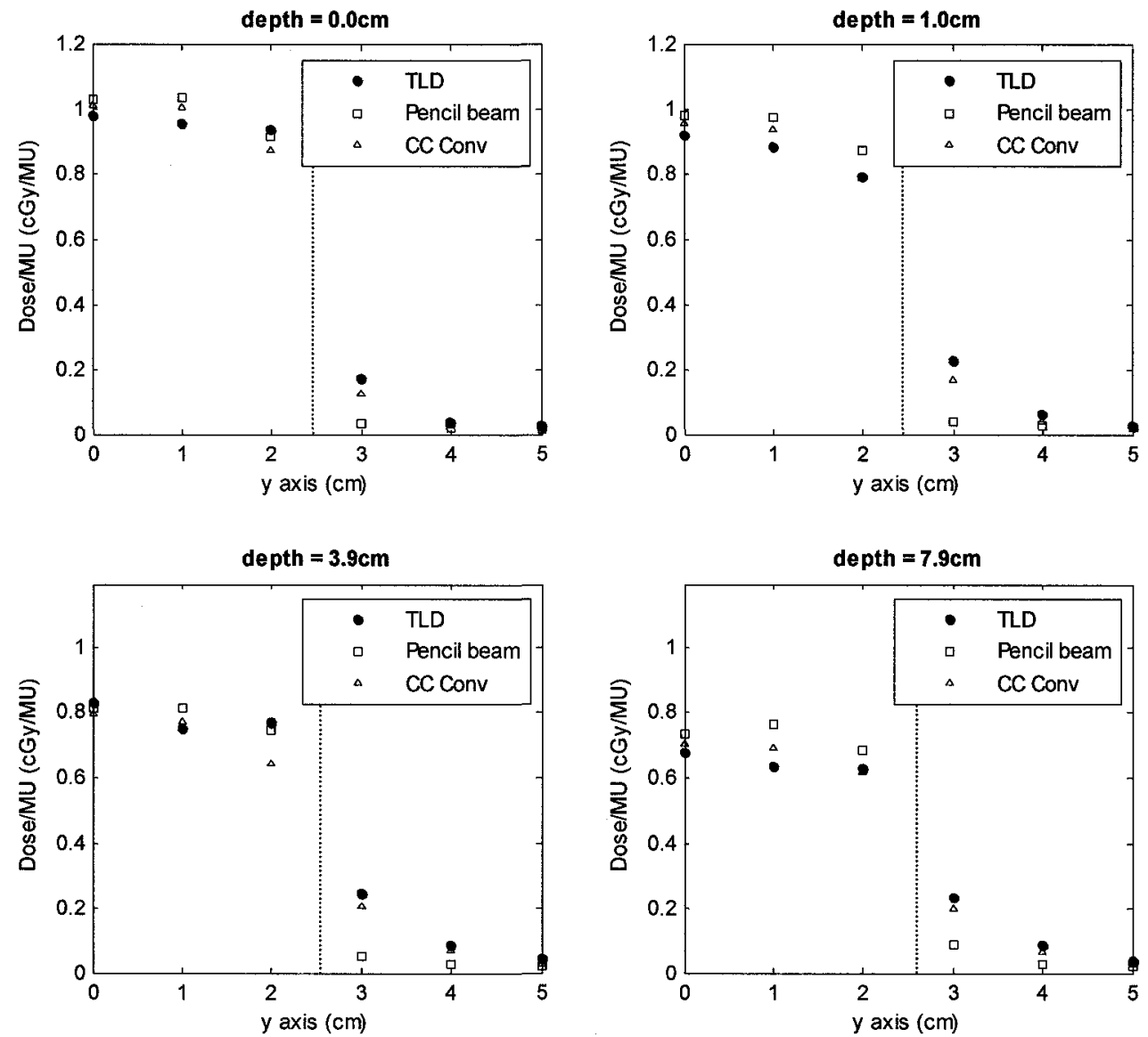

Figure 3.11 Measured (TLD) and calculated (PB and $\mathrm{CCC}$ ) dose profiles in lung equivalent material for a $10 \mathrm{MV}$ photon beam in the $5 \times 5 i i$ beam geometry at four depths below the proximal lung-acrylic interface (depths $=0.0 \mathrm{~cm}, 1.0 \mathrm{~cm}, 3.9 \mathrm{~cm}$ and $7.9 \mathrm{~cm}$ ). All points to the right of the dotted line lie within the primary (geometric) beam. The estimated TLD error is within the size of the symbols. 

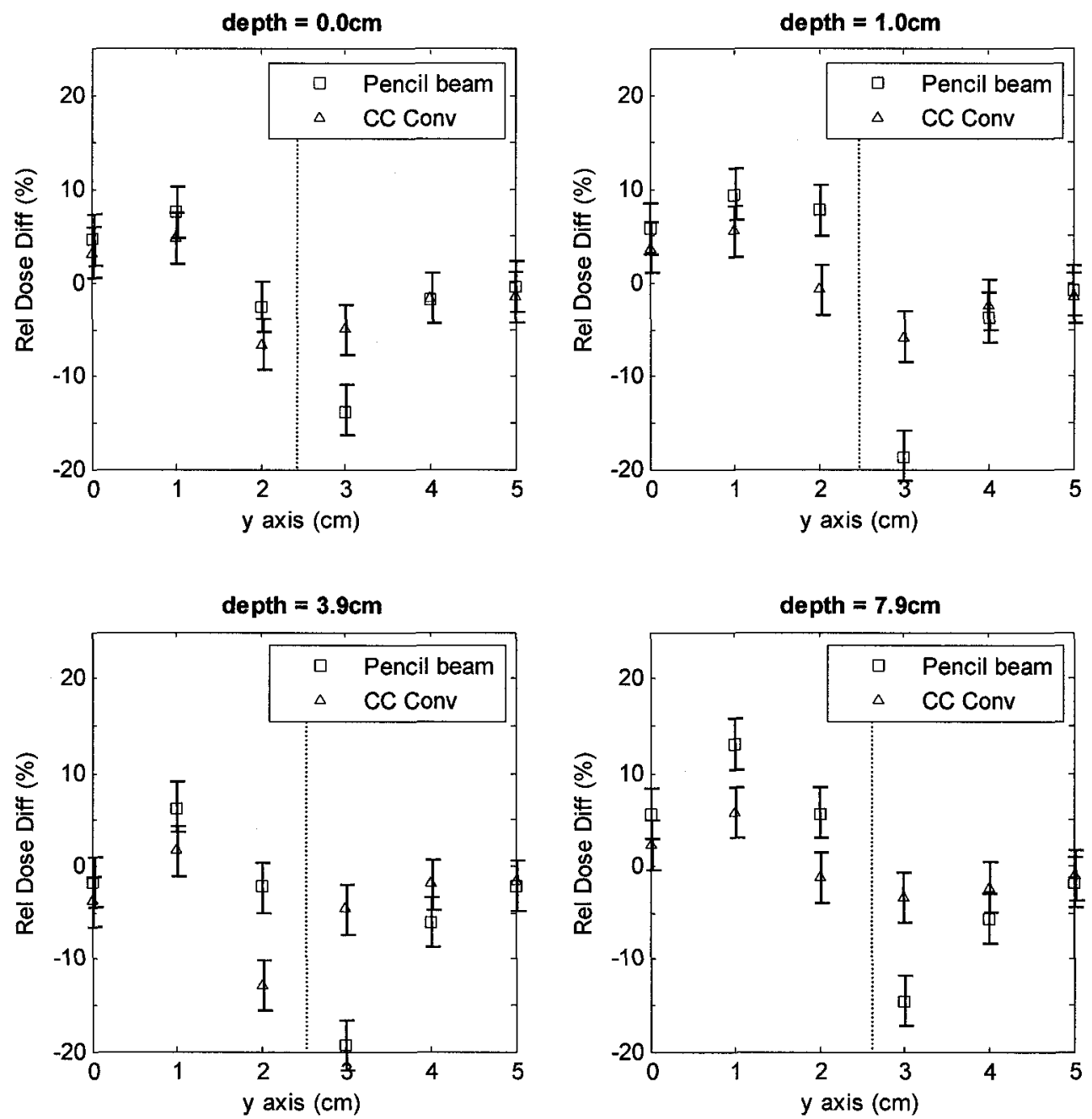

Figure 3.12 The $\%$ dose difference between the calculated (PB and CCC) and TLD measurements for a $10 \mathrm{MV}$ photon beam in the $5 \times 5 i i$ experimental geometry at four depths below the proximal lung-acrylic interface. All points to the right of the dotted line lie within the primary (geometric) beam. 

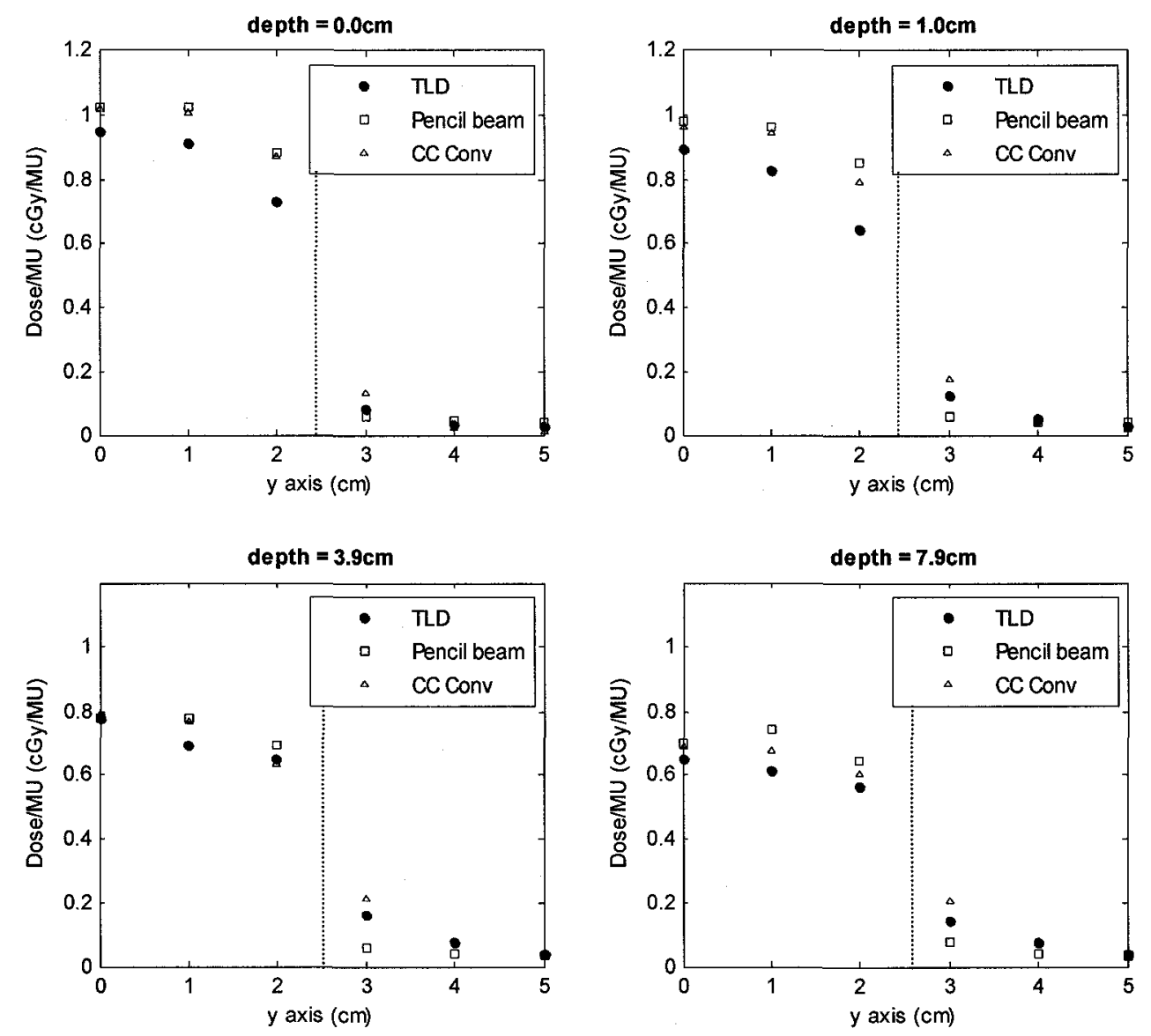

Figure 3.13 Measured (TLD) and calculated (PB and $\mathrm{CCC}$ ) dose profiles in lung equivalent material for a $18 \mathrm{MV}$ photon beam in the $5 \times 5 i i$ beam geometry at four depths below the proximal lung-acrylic interface (depths $=0.0 \mathrm{~cm}, 1.0 \mathrm{~cm}, 3.9 \mathrm{~cm}$ and $7.9 \mathrm{~cm}$ ). All points to the right of the dotted line lie within the primary (geometric) beam. The estimated TLD error is within the size of the symbols. 

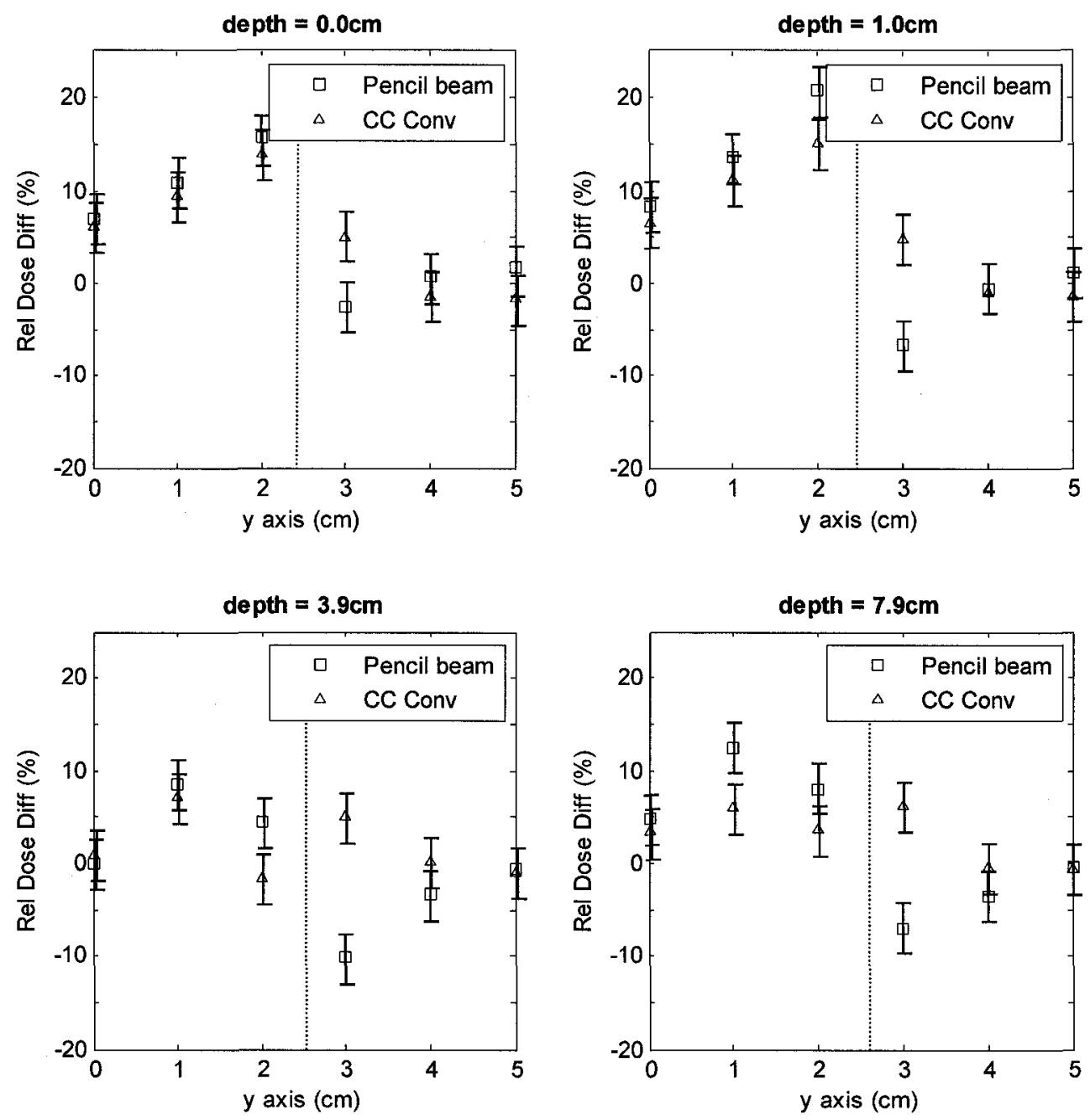

Figure 3.14 The \% dose difference between the calculated (PB and CCC) and TLD measurements for a $18 \mathrm{MV}$ photon beam in the $5 \times 5 i i$ experimental geometry at four depths below the proximal lung-acrylic interface. All points to the right of the dotted line lie within the primary (geometric) beam. 


\section{C. $10 \times 10$ beam geometry}

The $10 \times 10$ beam geometry is similar to the $5 \times 5 i$ beam geometry in that both contain lateral lung-acrylic interface walls within their fields. The phantom was designed to mimic a mediastinum with lungs to each side. The lung-acrylic interface lies at $x=-3.0$ $\mathrm{cm}$. The points to the right of the dotted line (geometric beam edge) all lie within the primary beam. For the 6,10 and $18 \mathrm{MV}$ beams, the dose profiles and difference curves are shown in Figures 3.15 to 3.20 . At distances of around $1.5 \mathrm{~cm}$ and greater outside the geometric beam edge, both algorithms agreed with measurement. Just outside the beam edge, the PB algorithm underestimated the dose by up to $9 \%$. At this same point, the $\mathrm{CCC}$ algorithm agreed with measurement at proximal and distal interfaces (depths $=0.0$ $\mathrm{cm}$ and $7.9 \mathrm{~cm}$ ) but over-predicted the dose towards the middle of the lung. At $0.5 \mathrm{~cm}$ inside the beam edge, the PB algorithm overestimated the dose. The CCC algorithm performed quite well at this point with the exception of a significant overestimation at the proximal interface. At $1.5 \mathrm{~cm}$ inside the primary beam, both algorithms calculated the dose reasonably well. 

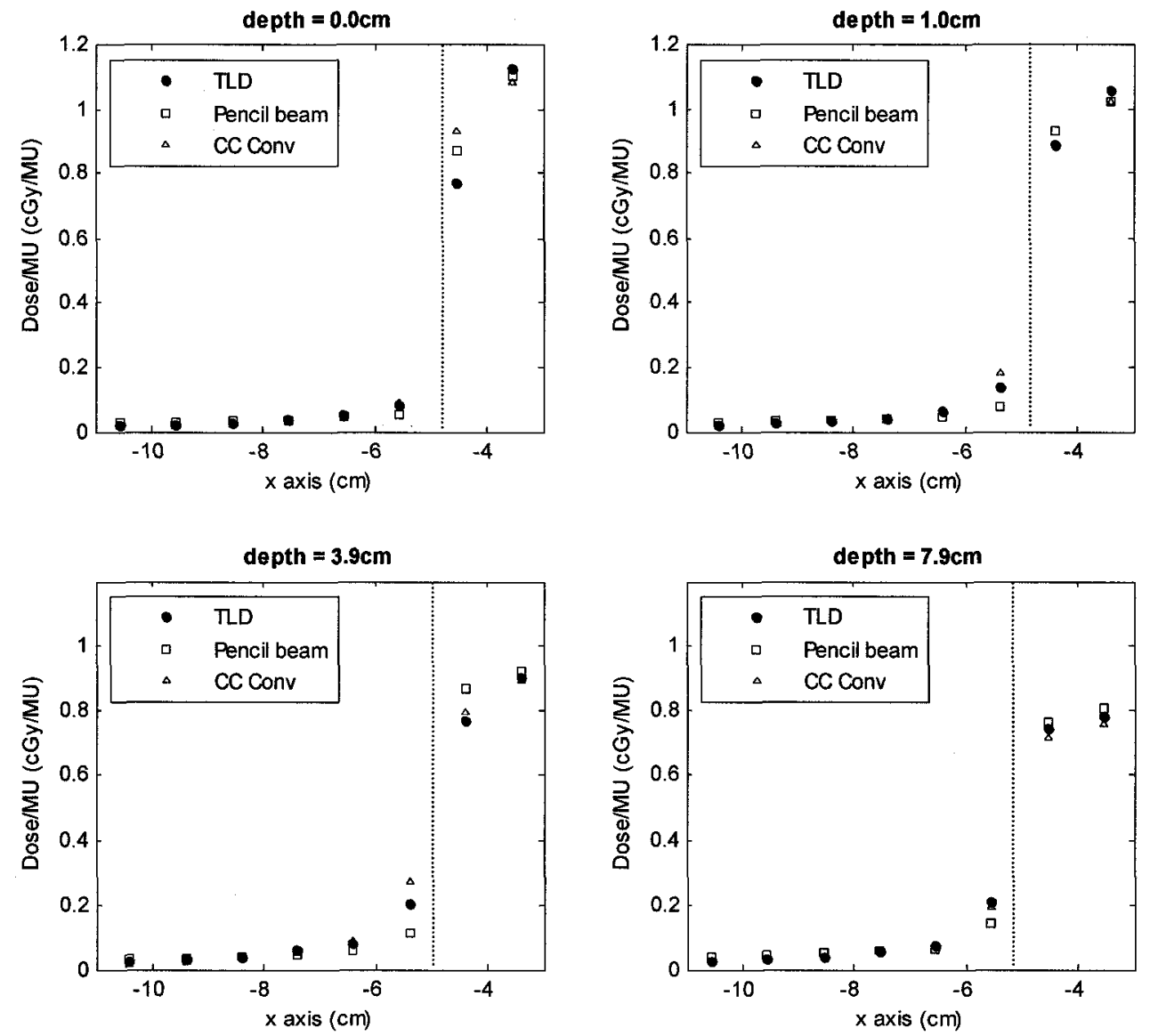

Figure 3.15 Measured (TLD) and calculated (PB and $\mathrm{CCC}$ ) dose profiles in lung equivalent material for a $6 \mathrm{MV}$ photon beam in the $10 \times 10$ beam geometry at four depths below the proximal lung-acrylic interface $($ depths $=0.0 \mathrm{~cm}, 1.0 \mathrm{~cm}, 3.9 \mathrm{~cm}$ and $7.9 \mathrm{~cm}$ ). All points to the right of the dotted line lie within the primary (geometric) beam. The estimated TLD error is within the size of the symbols. 

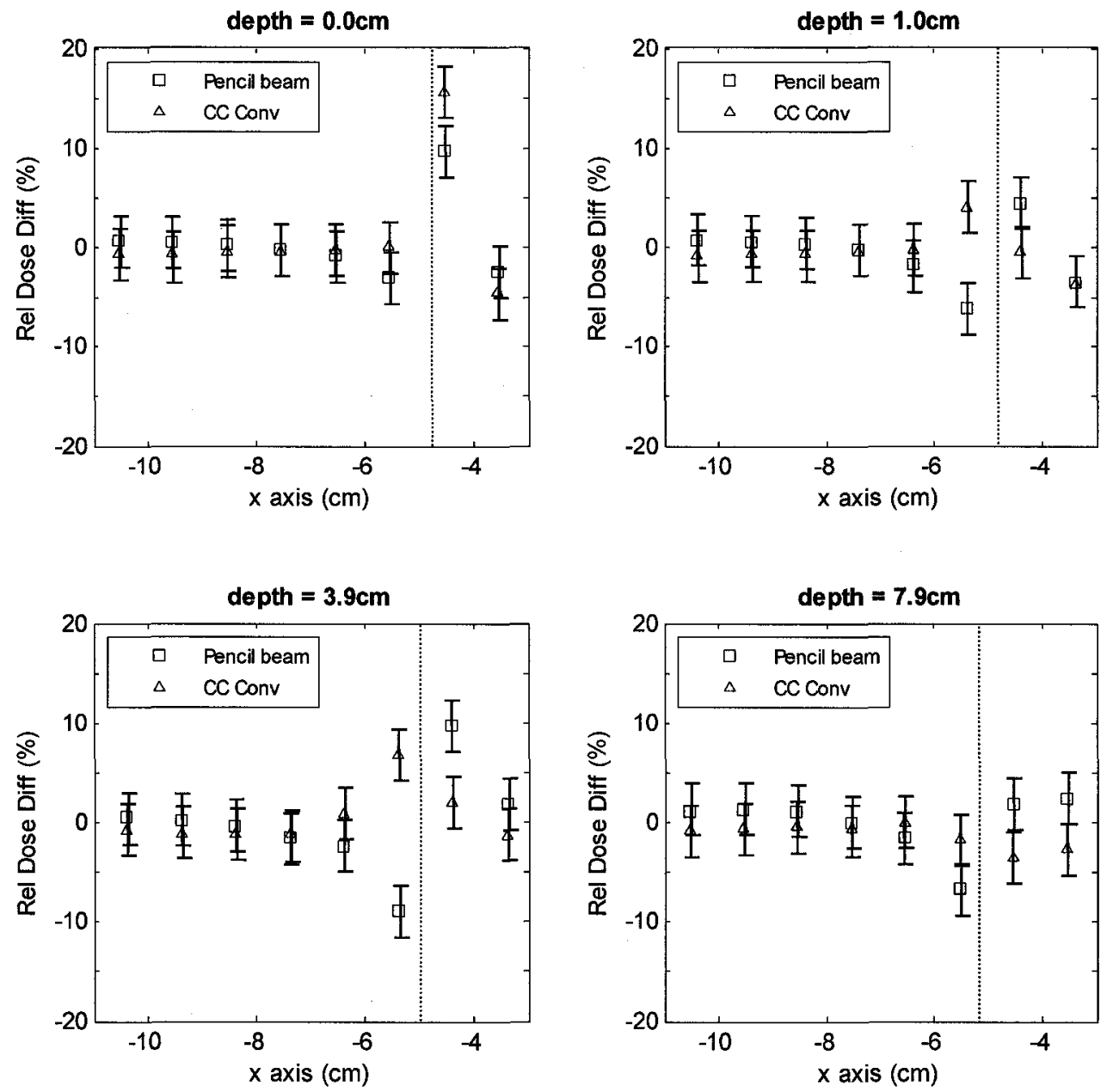

Figure 3.16 The \% dose difference between the calculated (PB and CCC) and TLD measurements for a $6 \mathrm{MV}$ photon beam in the $10 \times 10$ experimental geometry at four depths below the proximal lung-acrylic interface. All points to the right of the dotted line lie within the primary (geometric) beam. 

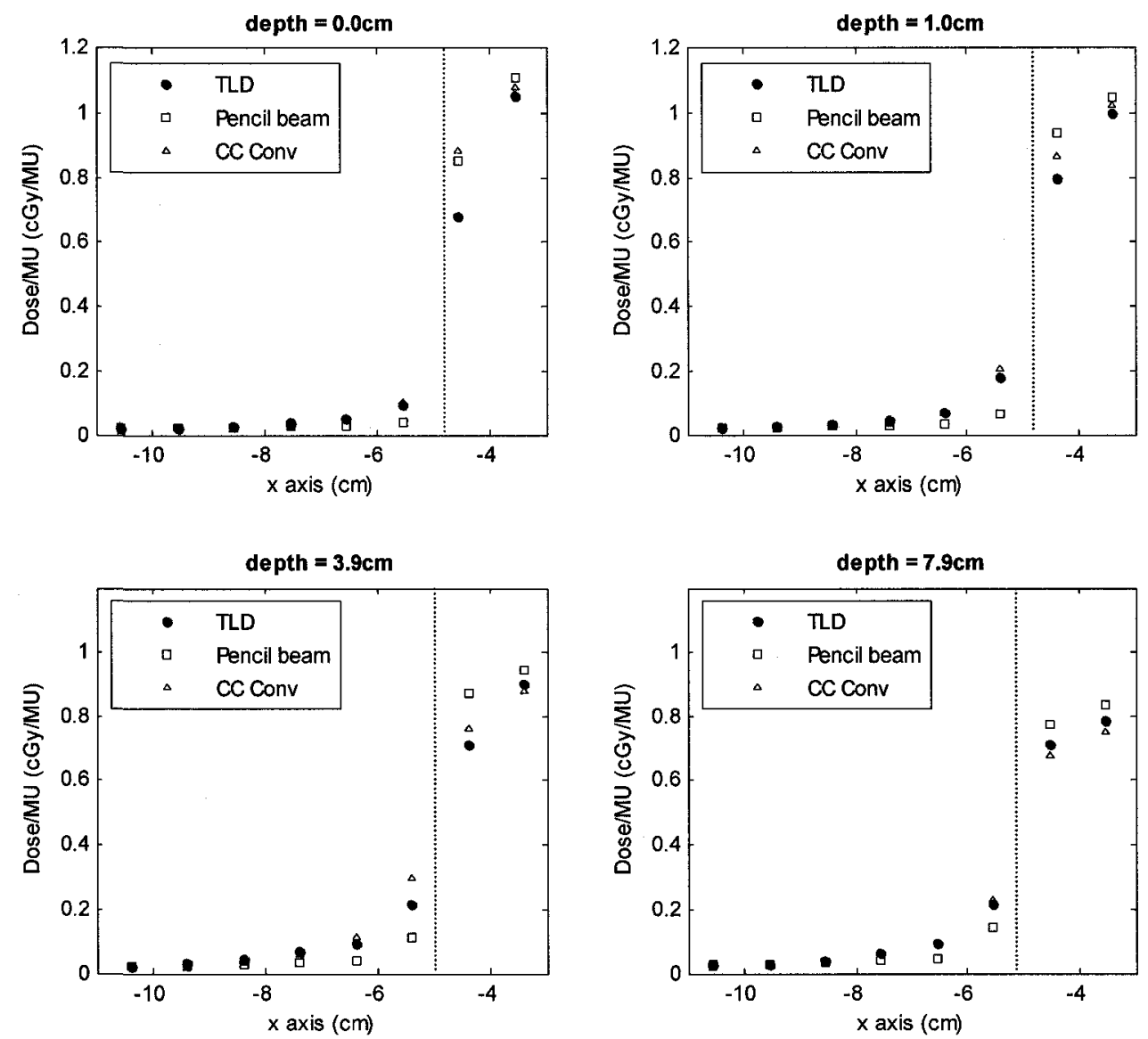

Figure 3.17 Measured (TLD) and calculated (PB and $\mathrm{CCC}$ ) dose profiles in lung equivalent material for a $10 \mathrm{MV}$ photon beam in the $10 \times 10$ beam geometry at four depths below the proximal lung-acrylic interface $($ depths $=0.0 \mathrm{~cm}, 1.0 \mathrm{~cm}, 3.9 \mathrm{~cm}$ and $7.9 \mathrm{~cm})$. All points to the right of the dotted line lie within the primary (geometric) beam. The estimated TLD error is within the size of the symbols. 

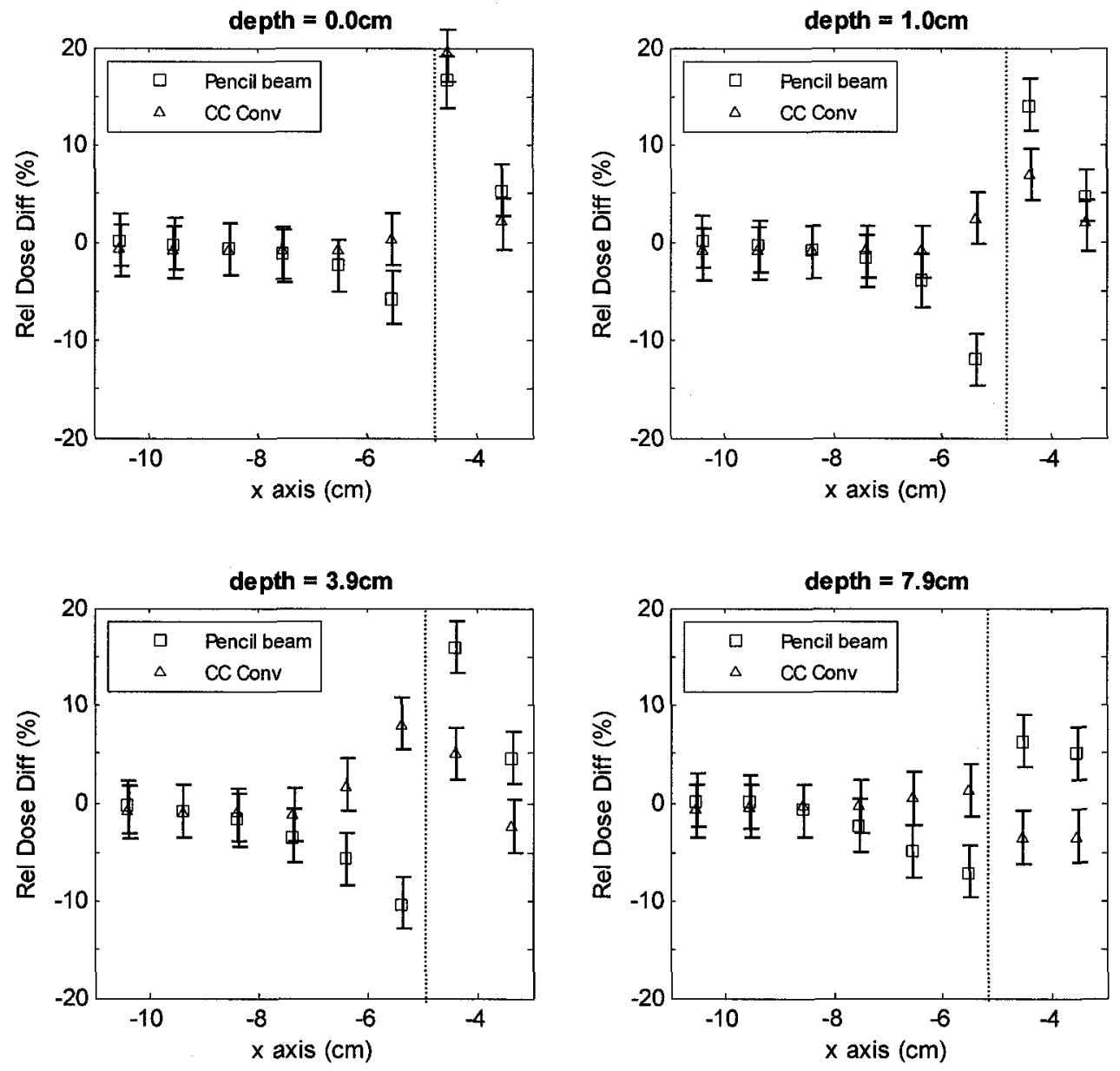

Figure 3.18 The \% dose difference between the calculated (PB and CCC) and TLD measurements for a $10 \mathrm{MV}$ photon beam in the $10 \times 10$ experimental geometry at four depths below the proximal lung-acrylic interface. All points to the right of the dotted line lie within the primary (geometric) beam. 

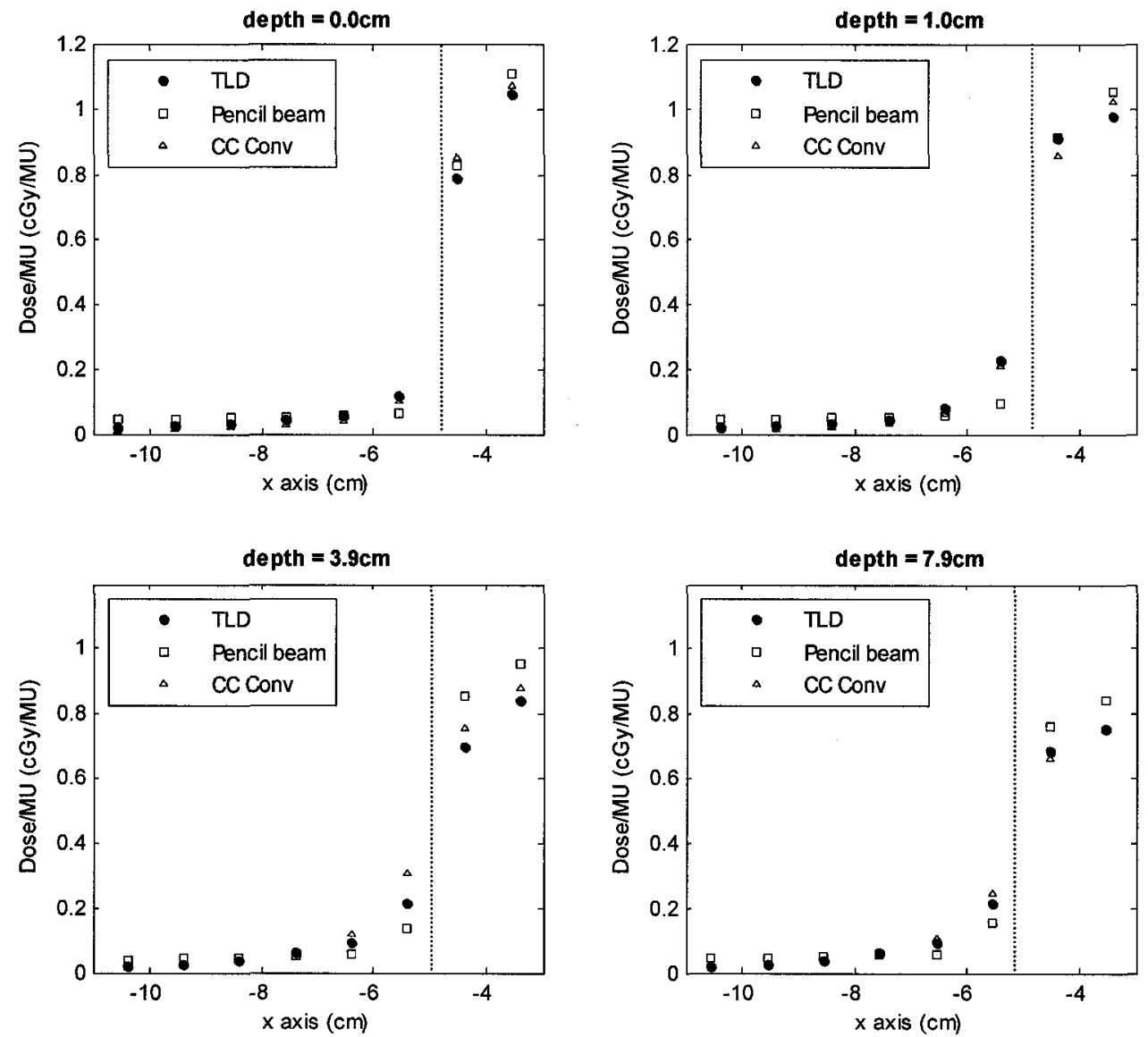

Figure 3.19 Measured (TLD) and calculated (PB and $\mathrm{CCC}$ ) dose profiles in lung equivalent material for a $18 \mathrm{MV}$ photon beam in the $10 \times 10$ beam geometry at four depths below the proximal lung-acrylic interface $($ depths $=0.0 \mathrm{~cm}, 1.0 \mathrm{~cm}, 3.9 \mathrm{~cm}$ and $7.9 \mathrm{~cm})$. All points to the right of the dotted line lie within the primary (geometric) beam. The estimated TLD error is within the size of the symbols. 

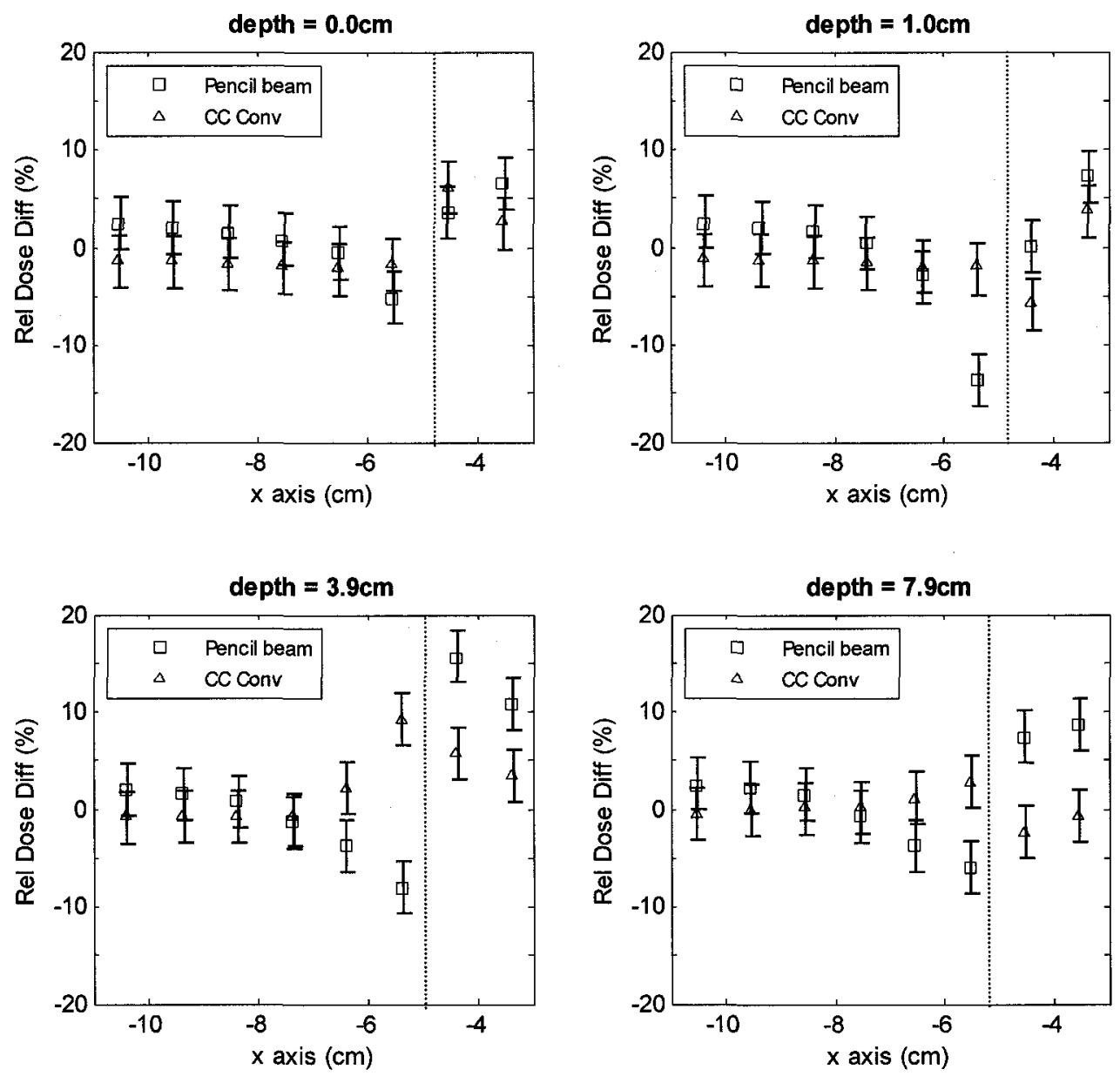

Figure 3.20 The \% dose difference between the calculated (PB and CCC) and TLD measurements for a $18 \mathrm{MV}$ photon beam in the $10 \times 10$ experimental geometry at four depths below the proximal lung-acrylic interface. All points to the right of the dotted line lie within the primary (geometric) beam. 


\section{Penumbra width as a function of energy and depth}

The penumbra width was defined as the distance between the $20 \%$ and $80 \%$ isodose lines. It is often used as a measure of the characteristic broadening of dose profiles in low density materials. For this chapter, we examined the penumbra width of the dose profiles for the $5 \times 5 i$ beam geometry. The dose profile data were fitted a curve by applying a piecewise cubic Hermite interpolation. This type of polynomial interpolation preserves monotonicity and the shape of the data, and is suitable for data with both "steep" and "flat" regions. It should be noted that this was not selected due to any physical basis, but rather just to give a reasonable estimate of the penumbra width. Figure 3.21 shows the penumbra width as a function of lung depth for three beam energies. For this geometry, the measurements show the penumbra width increasing (up to $15 \mathrm{~mm}$ ) between $0.0 \mathrm{~cm}$ and $4.0 \mathrm{~cm}$ and then decreasing near the distal interface. The CCC algorithm models this trend well, generally agreeing with the measured data for all three beam energies. The PB algorithm was not able to model the change in the penumbra width as a function of depth, underestimating the penumbra width at all depths and beam energies.

Our data (Figure 3.21) showed that the penumbra was broadest at a depth of approximately $4 \mathrm{~cm}$, midway through the lung-equivalent material. At this depth, we examined the energy-dependence of the penumbra width for the $5 \times 5 i$ geometry, as seen in Figure 3.22. The TLD measurements show that the penumbra width increases with increasing beam energy. The $\mathrm{CCC}$ algorithm predicted the broadening of the penumbra quite well. The PB calculated penumbra width showed little energy dependence and 
significantly under-predicted the broadening of the dose profiles. Similar results were seen for the other beam geometries. 
a)

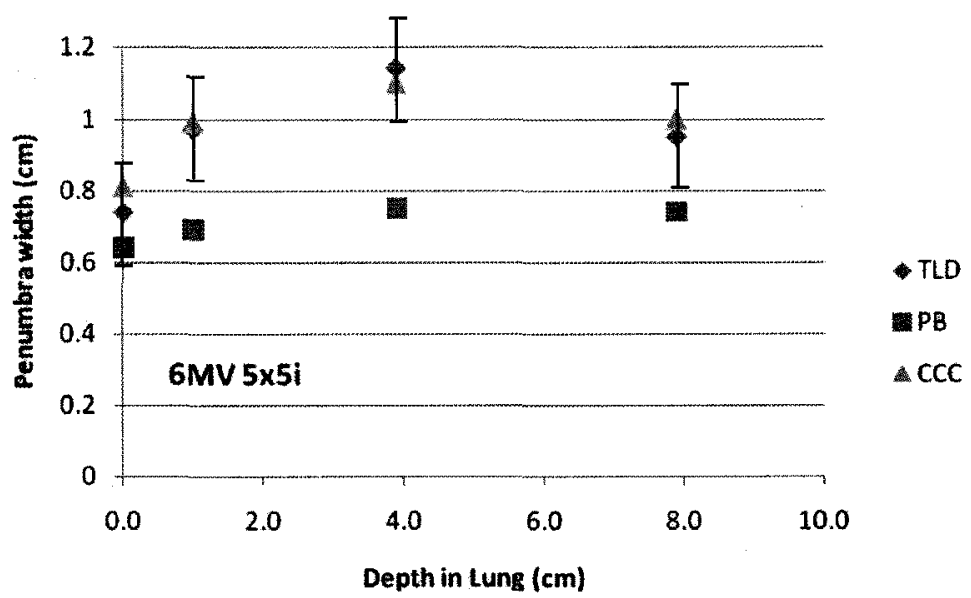

b)

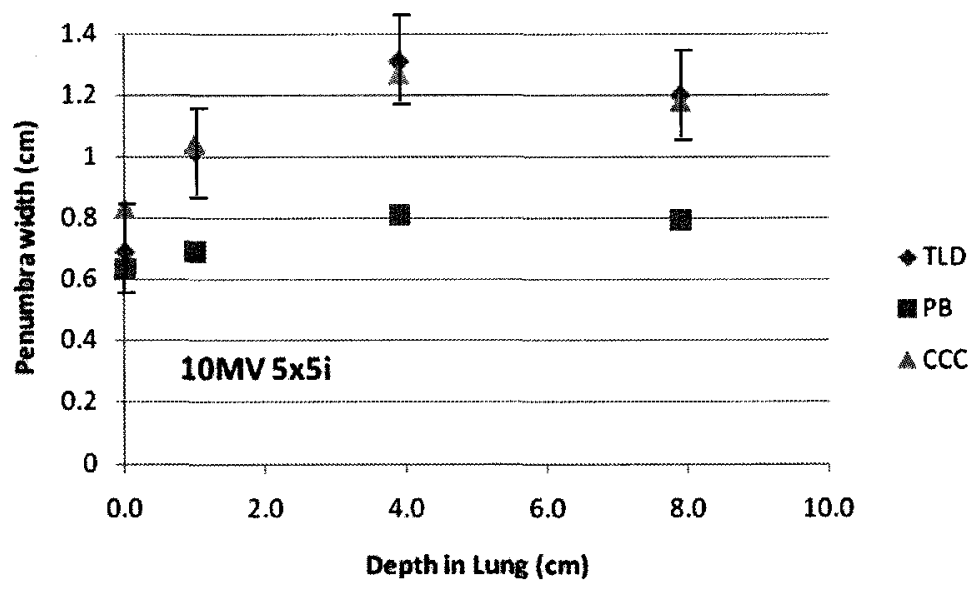

c)

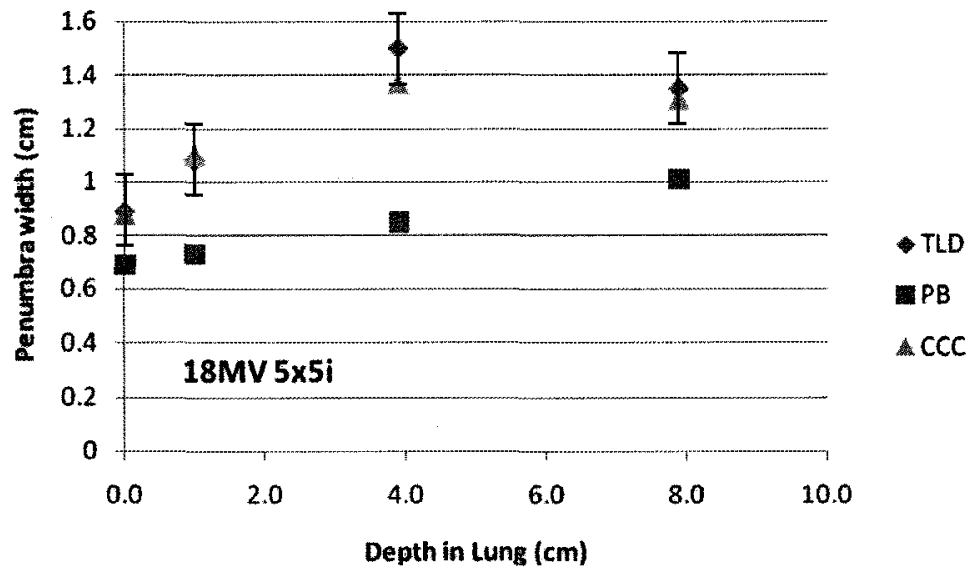

Figure 3.21 The penumbra width as a function of lung depth for the $5 \times 5 i$ geometry for a) $6 \mathrm{MV}$ b) $10 \mathrm{MV}$ and c) $18 \mathrm{MV}$ photon beams. The proximal and distal lung interfaces lie at depths of $0.0 \mathrm{~cm}$ and $8.0 \mathrm{~cm}$, respectively. 


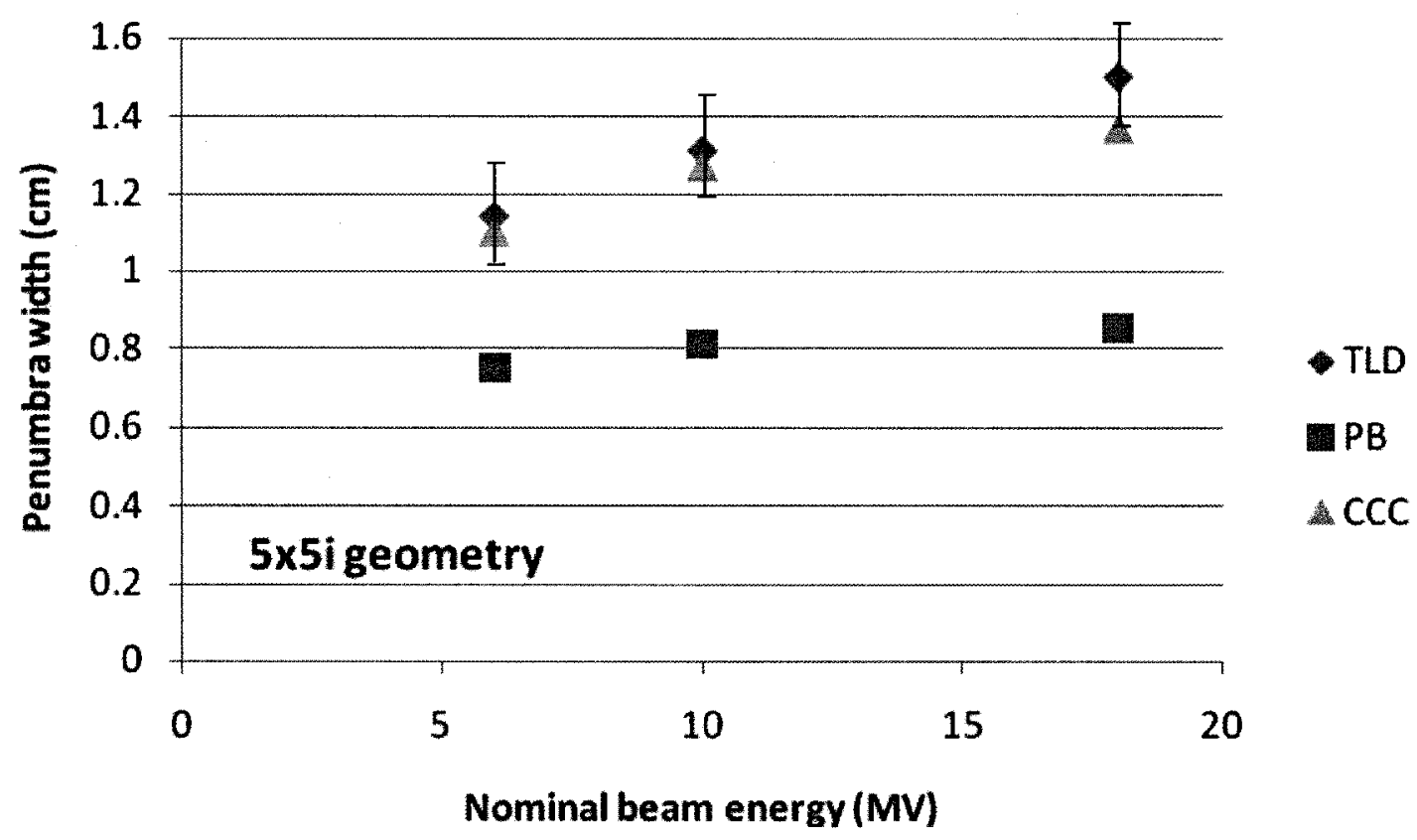

Figure 3.22 The penumbra width as a function of nominal beam energy for the $5 \times 5 i$ geometry at a depth midway $($ depth $=3.9 \mathrm{~cm})$ through the lung volume. 


\subsubsection{Discussion}

This study examined the performance of the PB and CCC algorithms as implemented on Theraplan Plus version 3.8 with Oncentra DCM version 2.0 in lung-equivalent material. The measured (TLD) profiles showed a broadening of the penumbra with increasing energy (Figure 3.22). This is a well-known effect due to the greater energy and subsequently larger range of the secondary electrons in the lower-density material (White et al 1996). The PB algorithm was not able to model this effect. This is not surprising considering that the PB algorithm utilizes a kernel that is not laterally scaled. As mentioned in section 2.2.1.2, the scatter dose is first calculated in homogeneous media and then corrected in inhomogeneous media along the beam path. This means that the heterogeneity correction (Equation 2.17) is in one dimension only and will not account for any lateral electron scatter. The end result is that depth-dose and profile characteristics in water are modeled well, but the model begins to fail in low density materials and near lung-acrylic interfaces. However, the CCC method correctly modeled the effect of the widening penumbra. The $\mathrm{CCC}$ algorithm approximates the dose kernel as a series of cones and so the transport of radiation is still performed in all three dimensions. This means that lateral electron scatter is accounted for in this algorithm. As mentioned in section 2.2.1.1, the main approximation is that the energy released within each cone is transported, attenuated and deposited only along its axis. As shown in our results, this limits the dose calculation accuracy of this algorithm near inhomogeneity interfaces where lateral electron disequilibrium exists. Our measurements showed that the relative dose error was generally less pronounced when no lateral interfaces were present, such as in the $5 \times 5 i i$ beam geometry. 
Another concern near the lung-acrylic interfaces is pixel averaging. The standard dose calculation grid for most treatment plans performed with Theraplan Plus creates a matrix of dose points spaced every $0.5 \mathrm{~cm}$. Since the dose distribution can fluctuate dramatically near interfaces, the TPS will tend to smooth out or misrepresent these perturbations. For our experiments, the dose profiles at depths of $0.0 \mathrm{~cm}$ and $7.9 \mathrm{~cm}$ in the lung lie directly at the proximal and distal lung-acrylic interfaces, respectively. At these interfaces, care must be taken to determine whether dose errors are a result of limitations in the algorithms or pixel averaging.

The use of acrylic for the lung phantom body can also introduce errors in the treatment planning dose calculation. TPSs will approximate all materials as water of varying density or a water-bone mix of varying density when performing dose calculations. This approximation has been shown to be acceptable for materials with density less than water. For the lung phantom, Theraplan Plus will treat the phantom body as a water-bone mix but with the electron density of acrylic $\left(\rho_{e}=1.14\right)$. As a result, the TPS will report the dose to water in acrylic because it does not know the mass density of the materials. This introduces a systematic error for all Theraplan Plus dose calculations for materials with densities greater than water. Fortunately for this study, the magnitude of this error is within a few percent. Additionally, all measurements performed within our experiments are within lung-equivalent material and we will not be reporting any TPS dose calculations within the acrylic body. Assuming the electron density is correct, the energy removed by the acrylic should be approximately correct. Therefore, the dose to the lung distal to acrylic should also be fairly correct. 
Many studies examining dose calculations in low-density materials show similar results to those shown in this section, though direct comparison is difficult due to differences in phantom materials and dimensions, beam geometries, depths, and photon energies. Most studies report dose errors in specific regions to evaluate dose calculation algorithms. However, Low et al (1998 and 2003) suggested the use of a gamma calculation for 2-D dose distribution comparison. Although this approach was not utilized in this chapter, adapting the methodology to a 1-D comparison could be useful for our data.

In 1994, Knöös et al investigated limitations of PB dose calculations in lung tissue, utilizing a phantom geometry similar to that used in our measurements. Deviations from Monte Carlo calculated dose values were small in unit-density materials but increased with beam energy in low-density material from $3 \%$ for $4 \mathrm{MV}$ to $14 \%$ for 18 MV x-rays. Figures 3.23 to 3.25 show relative dose differences between treatment planning calculations and TLD measurements as a function of nominal photon beam energy for the $5 \times 5 i, 5 \times 5 i i$ and $10 \times 10$ geometries, respectively. The dose data was taken from the plateau region of each profile at four depths below the proximal lung-acrylic interface. As before, the relative dose difference was calculated using equation 3.20. Our results show dose errors up to $13.5 \%$ for $18 \mathrm{MV}$ beams, agreeing with the results from Knöös et al (1994). Additionally, each figure shows that the relative dose difference increased with increasing nominal beam energy. 
Engelsman et al (2001) examined various inhomogeneity correction methods, including the PB algorithm with a 1-D correction, in a heterogeneous polystyrene and cork phantom. For a beam setup similar to the $5 \times 5 i i$ geometry, Engelsman showed that the PB calculated penumbra width did not vary with photon beam energy or depth in the low-density material. Our PB calculations also showed minimal depth and energy dependencies as seen in Figures 3.21 and 3.22, respectively. They also found that the PB calculations over-estimated the dose along the central axis by up to $6.5 \%$ depending on beam energy and depth in the low-density material. Cranmer-Sargison et al (2004) examined the PB calculated dose in the presence of a vertical water-lung interface similar to that used in our study. When compared to film measurements, the PB calculated dose for a $4 \times 4 \mathrm{~cm}^{2}$ field was up to $15 \%$ greater in the lung region. Comparing these results to our $5 \times 5 i$ experiments which include a similar interface, the PB calculated dose was up to $10 \%$ and $13 \%$ greater in the lung region at $0.5 \mathrm{~cm}$ and $1.5 \mathrm{~cm}$ away from the interface, respectively.

In 2004, Carrasco et al compared the dose calculated by several treatment planning algorithms in phantoms with lung-equivalent heterogeneities under conditions of lateral electronic disequilibrium. The PB penumbra width of a $5 \times 5 \mathrm{~cm}^{2}$ field at 6 and $18 \mathrm{MV}$ photon energies and $10 \mathrm{~cm}$ depth was 4.1 and $6.2 \mathrm{~mm}$, respectively. For identical conditions, the CCC penumbra width was 9.9 and $15.1 \mathrm{~mm}$, respectively. The average deviation between measured and calculated along the central axis beam dose in the lungequivalent material increased with decreasing field size and was found to be a maximum 


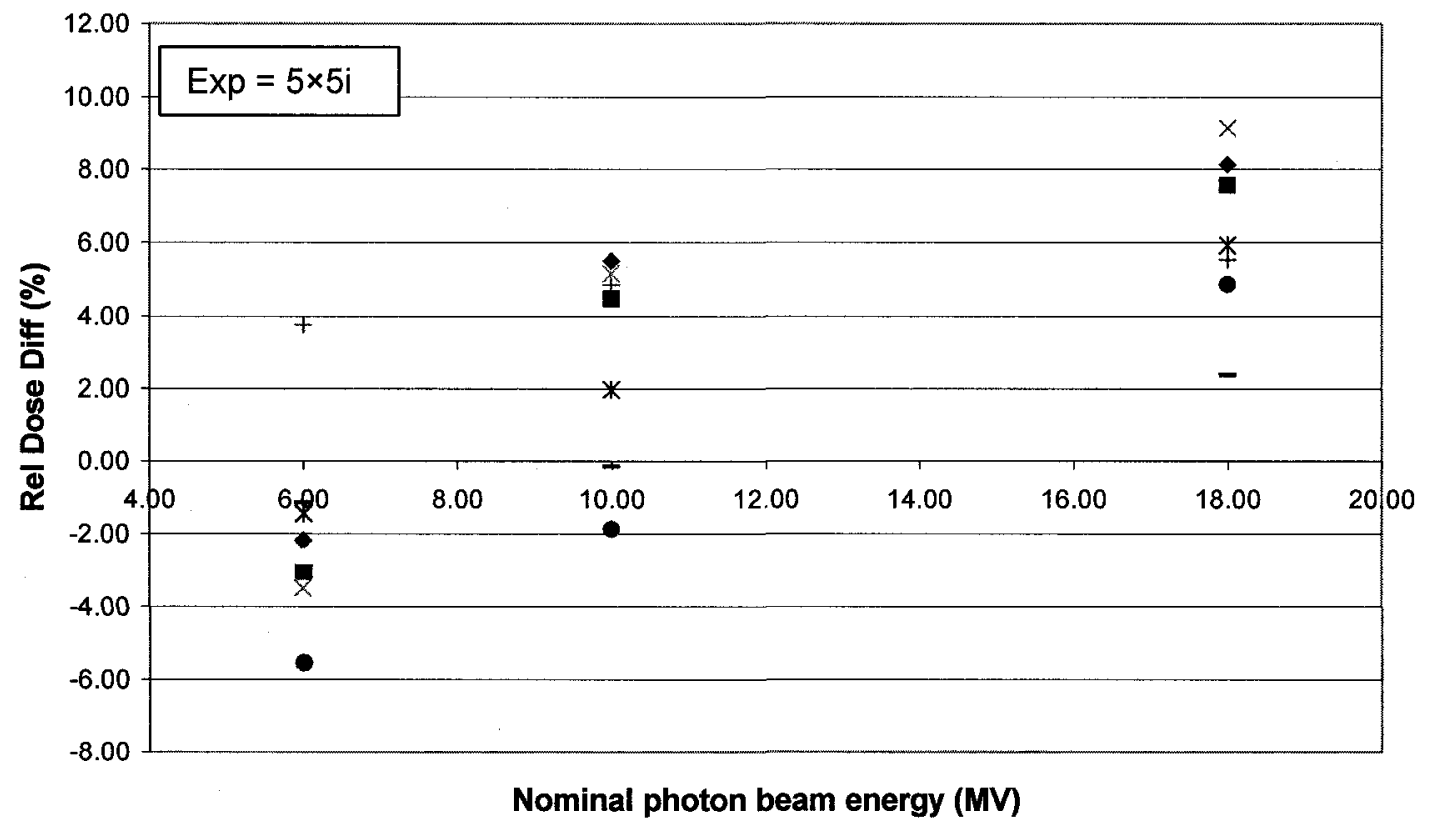

- depth $=0.0 \mathrm{~cm}$ pencil beam

depth $=0.0 \mathrm{~cm} \mathrm{CCC} \mathrm{depth}=1.0 \mathrm{~cm}$ pencil beam $\times$ depth $=1.0 \mathrm{~cm} \mathrm{CCC}$ $*$ depth $=3.9 \mathrm{~cm}$ pencil beam

depth $=3.9 \mathrm{~cm} \mathrm{CCC}+$ depth $=7.9 \mathrm{~cm}$ pencil beam - depth $=7.9 \mathrm{~cm} \mathrm{CCC}$

Figure 3.23 Relative dose difference between treatment planning calculations (PB and CCC algorithms) and TLD measurements as a function of nominal photon beam energy with the $5 \times 5 i$ geometry at four depths below the proximal lung-acrylic interface. All data points were measured in the plateau region, the dose point nearest the central axis of the geometric beam. The relative error on all measurements is estimated at $2.8 \%$. 


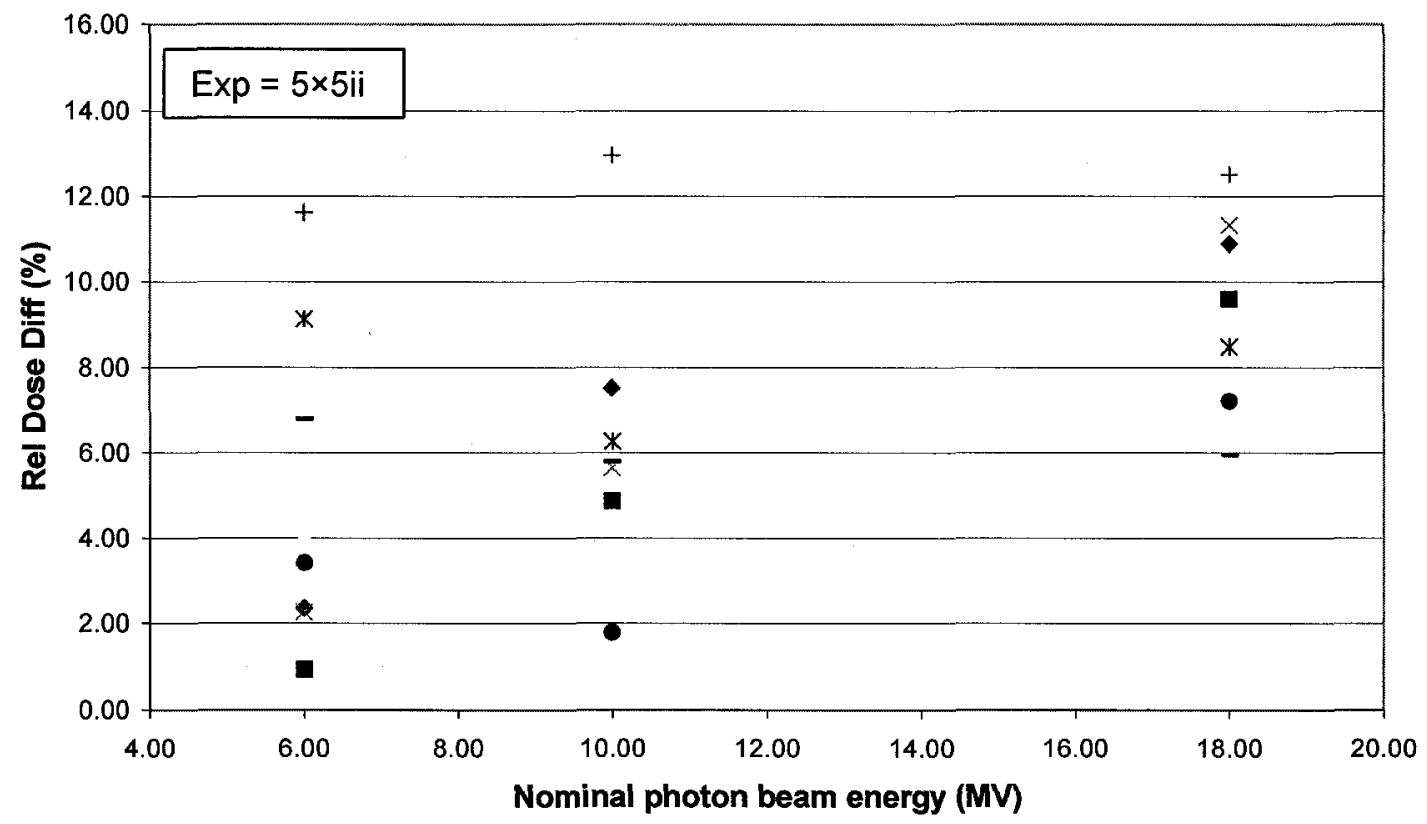

depth $=0.0 \mathrm{~cm}$ pencil beam

* depth $=3.9 \mathrm{~cm}$ pencil beam

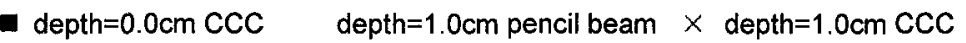

- depth $=3.9 \mathrm{~cm} \mathrm{CCC}+$ depth $=7.9 \mathrm{~cm}$ pencil beam - depth $=7.9 \mathrm{~cm} \mathrm{CCC}$

Figure 3.24 Relative dose difference between treatment planning calculations (PB and CCC algorithms) and TLD measurements as a function of nominal photon beam energy with the $5 \times 5 i i$ geometry at four depths below the proximal lung-acrylic interface. All data points were measured in the plateau region, the dose point nearest the central axis of the geometric beam. The relative error on all measurements is estimated at $2.8 \%$. 


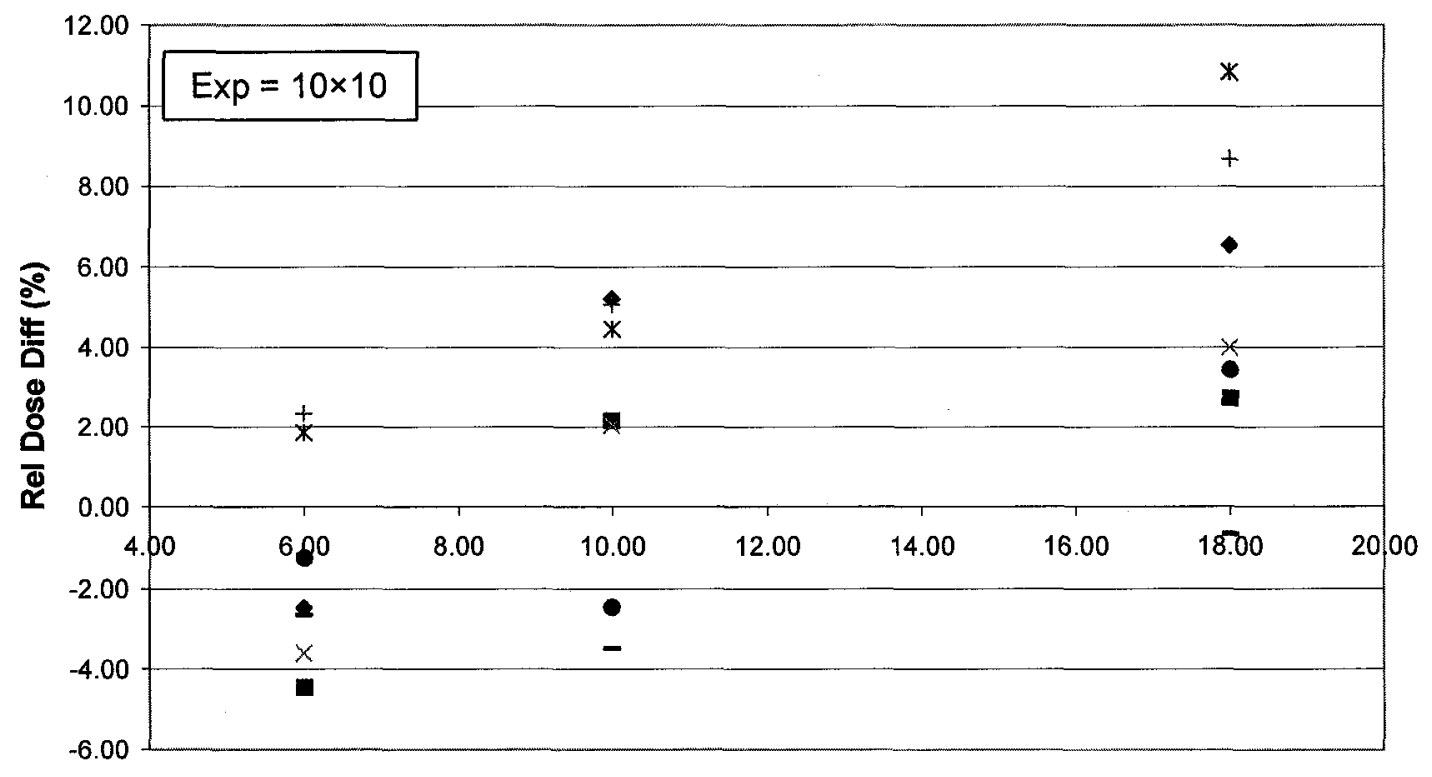

Nominal photon beam energy (MV)

- depth $=0.0 \mathrm{~cm}$ pencil beam

Figure 3.25 Relative dose difference between treatment planning calculations (PB and CCC algorithms) and TLD measurements as a function of nominal photon beam energy with the $10 \times 10$ geometry at four depths below the proximal lung-acrylic interface. All data points were measured in the plateau region, the dose point nearest the central axis of the geometric beam. The relative error on all measurements is estimated at $2.8 \%$. 
just below the proximal lung-water interface. As can be seen from our data (Figures 3.21 ), the $\mathrm{PB}$ calculated penumbra width of a $5 \times 5 \mathrm{~cm}^{2}$ field for 6 and $18 \mathrm{MV}$ photon beams at $7.9 \mathrm{~cm}$ depth was $7.5 \mathrm{~mm}$ and $10.0 \mathrm{~mm}$, respectively. The CCC calculated the penumbra width for the same conditions to be 10.0 and $13.0 \mathrm{~mm}$, respectively. However, it should be noted that our measurements were taken near lateral and distal lung-acrylic interfaces.

Butson et al (2000) examined dose calculations with the CCC method in an anthropomorphic lung phantom. Differences in calculated and measured dose were up to $5 \%$ at a point within solid water but approximately $1 \mathrm{~mm}$ from the lung-solid water boundary. This highlights the impact of lateral electron transport on both sides of the lung-water interface. Metcalfe et al (1993) performed a similar comparison but within the lung-equivalent material itself, revealing that the CCC method overestimated the midlung dose for a $5 \times 5 \mathrm{~cm}^{2}$ field by $7.2 \%(6 \mathrm{MV})$ and $6.4 \%(10 \mathrm{MV})$. In 2000, Arnfield et al also investigated the impact of electron transport on the accuracy of calculated dose in a heterogeneous lung and water phantom. The CCC calculated penumbra width for a $4 \times 4$ $\mathrm{cm}^{2}$ field at the distal lung-water interface was found to be 6.0 and $10.0 \mathrm{~mm}$ for 6 and 18 MV photon energies, respectively. In comparing the dose calculated by $\mathrm{CCC}$ and MC methods, the CCC method was found to over-predict the dose for several centimeters beyond the proximal lung-water interface, similar to the trends shown by our data. Such tendencies are commonly observed in CCC models evaluating dose beyond a high-to-low density interface, especially for small field sizes and high energy beams (Woo and Cunningham 1990). 
Mah and Van Dyk (1991) studied the clinical impact of measured and calculated dose discrepancies due to low-density inhomogeneities. In studying 100 clinical thoracic cases, it was found that using doses uncorrected for inhomogeneities resulted in an underestimation of the risk of radiological lung damage by up to $19 \%$. Lung toxicity associated with radiation treatments is a major concern and oncologists utilize several toxicity risk parameters to help set dose limits in the lung. Dose-volume histograms (DVHs) report the volume of tissue receiving a certain amount of dose. They are now used as a standard plan evaluation tool. Graham et al (1999) and Hernando et al (2001) reported that specific DVH parameters were a measure of risk for radiation pneumonitis in NSCLC (non-small cell lung cancer) patients treated with radiotherapy. Volume dose, specifically V20 and V30, were identified as useful parameters to reduce the risk of radiation-induced pneumonitis. V20 and V30 represent the percentage of irradiated lung volume exceeding $20 \mathrm{~Gy}$ and $30 \mathrm{~Gy}$, respectively. Graham et al (1999) and Hernando et al (2001) suggested that V20 and V30 not exceed 30\% and 20\%, respectively. Allen et al (2006) investigated fatal pneumonitis as a result of IMRT treatment for mesothelioma. From a pool of 13 patients, they reported that the median V20 and V5 (volume of lung receiving $5 \mathrm{~Gy}$ or more) for the patients who developed pneumonitis were $17.6 \%$ and $98.6 \%$, respectively. The median values for patients who did not develop pneumonitis was $10.9 \%$ and $90.0 \%$, respectively. Their data suggested that for IMRT, metrics such as V5 and V20 be used for tolerance levels in future patients.

We found that the PB algorithm underestimates the dose outside the geometric field edge. This means that, depending on beam geometry and dose per beam, the 
volume of V5, V20 or even V30 can be significantly underestimated. Thus patients could have significant normal tissue complication pneumonitis that physicians would not expect based on the DVH curve presented to them from the planning system.

Dose errors within the primary beam present different clinical concerns, since the beam is expected to be incident on the target (tumour cells). Our results also showed that the PB and CCC methods can overestimate the dose by up to 13.6 and $11.3 \%$ in the plateau region (for the $5 \times 5 i$ ii beam geometry, with an $18 \mathrm{MV}$ photon beam at a depth of 1 $\mathrm{cm}$ below the proximal lung-acrylic interface). When the TPS overestimates the predicted dose, the result may be an under-treatment of the tumour. Over the course of multiple fractions, this dose error can impact the success or failure of the treatment.

\subsubsection{Conclusions}

The accuracy of PB and CCC algorithms in low-density materials has been extensively investigated. The focus of this study was to examine the Theraplan Plus v3.8 (with Oncentra DCM v2.0) implementation of these algorithms in lung-equivalent material. We found that the Oncentra implementation of these algorithms was similar to implementations in other planning systems as reported in the literature, and that the performance of these algorithms was consistent with what would be expected from knowledge of how the algorithms work. The models have been shown to work well in unit density material but have problems in low-density materials. 
Measurements showed that the penumbra width increased with increasing energy, a trend that was not modeled by the PB algorithm. The CCC algorithm showed consistently better results than the PB technique. Although the CCC method was not able to model the broadening of the penumbra with increasing energy to the extent shown with measurements, the general trend was still evident. The greatest dose errors were observed for fields including lateral lung-acrylic interfaces, creating large regions of lateral electron disequilibrium and highlighting the lack of electron transport modeled in the algorithms. For these reasons, it is not recommended to use high energy beams with small fields in the lung.

The first clinical implication of these results is that when the TPS overestimates the dose, the treatment success rate can drop as a result of reduced dose to the tumour. A second implication is that when the TPS underestimates the dose, there can be an increase in lung toxicity as a result of exceeding V20 and V30 limits. It is important that the planning systems be used with care and that oncologist and users are aware of the limitations. 


\section{Chapter 4}

\section{Treatment dose errors due to beam attenuation by a carbon fiber tabletop}

\subsection{Abstract}

Carbon fiber is commonly used in radiation therapy for treatment tabletops and various immobilization and support devices, partially because it is generally perceived to be almost radiotransparent to high-energy photons. To avoid exposure to normal tissue during modern radiation therapy, one must deliver the radiation from all gantry angles; hence, beams often transit the couch proximal to the patient. The effects of the beam attenuation by the support structure of the couch are often neglected in the planning process. In this study, we investigate the attenuation of 6-MV and 18-MV photon beams by a Medtec (Orange City, IA) carbon fiber couch. We have determined that neglecting the attenuation of oblique treatment fields by the carbon fiber couch can result in 
localized dose reduction from $4 \%$ to $16 \%$, depending on energy, field size, and geometry. Further, we investigate the ability of a commercial treatment-planning system (Theraplan Plus v3.8) to account for the attenuation by the treatment couch. Results show that incorporating the carbon fiber couch in the patient model reduces the dose error to less than $2 \%$. The variation in dose reduction as a function of longitudinal couch position was also measured. In the triangular strut region of the couch, the attenuation varied $\pm 0.5 \%$ following the periodic nature of the support structure. Based on these findings, we propose the routine incorporation of the treatment tabletop into patient treatment planning dose calculations.

\subsection{Introduction}

The growing use of carbon fiber materials in radiation therapy is largely due to their high mechanical strength, low specific density, and radio-translucence (de Mooy 1991). These characteristics make carbon fiber materials ideal for the patient support assembly. Prior to the use of these materials for treatment couches, the patient support structure consisted of a table mounted on steel rails or stabilized with a steel spine down its center. For various gantry angles, these high-density rails have the potential to attenuate the beam by more than $40 \%$, making it an obvious obstacle to avoid during patient setup. Consequently, geometric methodologies were proposed to ensure that beam-couch intersections were detected and dealt with appropriately (Meyer et al 2001, Muthuswamy et al 1999, Yorke 1989). 
The benefits of carbon fiber over steel alloys make the new material a welcome change in radiotherapy practice, but because of the general assumption that attenuation by the couch is negligible, the strategies developed to avoid the couch are now often ignored. There is still little literature regarding the effects of carbon fiber patient support structures on clinical dose distribution. In 1991, de Mooy characterized the properties of carbon fiber and introduced applications of the material for use in radiation therapy. Meara and Langmack (1998) added to this characterization by investigating the transmission and buildup characteristics in 5-MV, 6-MV, and 8-MV beams for thin panels of carbon fiber in combination with a variety of other plastics.

The majority of subsequent publications have focused on attenuation, buildup, and increased skin dose caused by immobilization devices and table inserts. de Ost et al (1997) observed 1\% attenuation through commercial carbon inserts in Co-60 and 6-MV beams, but also noted that the surface dose increased from $18 \%$ to $77 \%$ of maximum dose, and $21 \%$ to $55 \%$ of maximum dose, respectively. Higgins et al (2001) validated de Ost et al's results and concluded that there is minimal attenuation due to the inserts but added that the magnitude of the increase in surface dose was relatively larger for smaller beam sizes. Butson et al (2002) quoted similar results for a 6-MV photon beam. Carl and Vestergaard (2000) suggested that carbon fiber materials with thicknesses greater than $100 \mathrm{mg} / \mathrm{cm}^{2}$ should be avoided for 4-MV beams to reduce skin dose in cases when the cumulative dose exceeds 54 Gy to $60 \mathrm{~Gy}$. 
McCormack et al (2005) extended previously published results by examining beam attenuation by a carbon fiber couch insert at various gantry angles. It was found that a $6-\mathrm{MV}$ photon beam was attenuated $2 \%$ at normal incidence and up to $9 \%$ at oblique angles. In 2003, Vieira et al quantified the effect of carbon fiber treatment couch rails in combination with assorted immobilization devices on beam attenuation, reporting up to $15 \%$ attenuation of a 6-MV photon beam during head and neck treatments.

In this study, the attenuation of photon beams traversing obliquely through the carbon fiber support rails of a clinical treatment couch were examined. Attenuation of beam fluence was measured in-air at two locations along the treatment couch under various conditions for both 6-MV and 18-MV photon beams. Dose reduction measurements were also made in-phantom. By including the carbon fiber couch in the CT image of the phantom, the effect of its attenuation on dose distribution was calculated with a commercial treatment-planning system, Theraplan Plus v3.8 (Nucletron, Ottawa, ON, Canada). The calculations were compared to measured data to investigate the ability of the planning system to properly model the couch attenuation

\subsection{Materials and Methods}

All measurements were performed on a Siemens Mevatron linear accelerator at The Ottawa Hospital Regional Cancer Centre. The treatment unit couch top was a Medtec indexed patient positioning system (IPPS ${ }^{\mathrm{TM}}$ ) constructed with carbon fiber rails and grid panels. Measurements were made in-air and in-phantom using the geometry shown in Fig. 4.1. The in-air measurements were made with an RK Chamber (model 8305) and 
Keithley Therapy Dosimeter (model 35040). For in-air measurements, $0.4 \mathrm{~cm}$ and 0.8 $\mathrm{cm}$ thick brass caps provided buildup for 6-MV and 18-MV photon beam energies, respectively. The in-phantom dose measurements were made at the center of a $20 \times 20 \times$ $20 \mathrm{~cm}^{3}$ acrylic block phantom with a NE 2571 ion chamber and NE 2570 electrometer.

Attenuation was calculated by the ratio of the measurements taken under the two conditions shown in Fig. 4.1. The measurements were made with and without the carbon fiber couch rails attenuating the beam for $5 \times 5 \mathrm{~cm}^{2}$ and $10 \times 10 \mathrm{~cm}^{2}$ field sizes at both 6MV and 18-MV photon energies delivering 100 monitor units. In all cases, the gantry angle was $225^{\circ}$, a beam orientation commonly used in standard radiation therapy and an angle that maximizes the thickness of carbon fiber material between the beam source and point of measurement.

Figure 4.2 shows the longitudinal intersection points of the beam central axis with the couch. The in-air measurements were taken at locations A and C, representing fields transiting through the solid and strut regions of the couch, respectively. The in-phantom measurements were all taken at a location within the grid region between positions B and D. To investigate the longitudinal dependence of the beam attenuation, the experimental setup was shifted along the longitudinal axis of the treatment couch in increments of $1 \mathrm{~cm}$ beginning at location $\mathrm{B}$ and ending at location $\mathrm{D}$.

The experiment described above was also modeled in Theraplan Plus version 3.8 (pencil beam algorithm). A CT dataset ( $3 \mathrm{~mm}$ slice thickness with $3 \mathrm{~mm}$ spacing) of the 
entire phantom sitting on the Medtec therapy couch as shown in Fig. 4.1(a) was acquired on the Philips AcQsim large bore, single-slice CT simulator (Philips Medical Systems, ON, Canada). The entire cross section of the treatment couch was included on each slice. The DICOM RT images were transferred to the treatment-planning system, where dose calculations were performed using the standard Theraplan dose calculation model with inhomogeneity correction turned on. Calculations were performed for two cases: the first where the Med-Tec couch was excluded from the calculation matrix and the second where it was included. In both cases, the CT tabletop was excluded from the treatmentplanning calculations. 


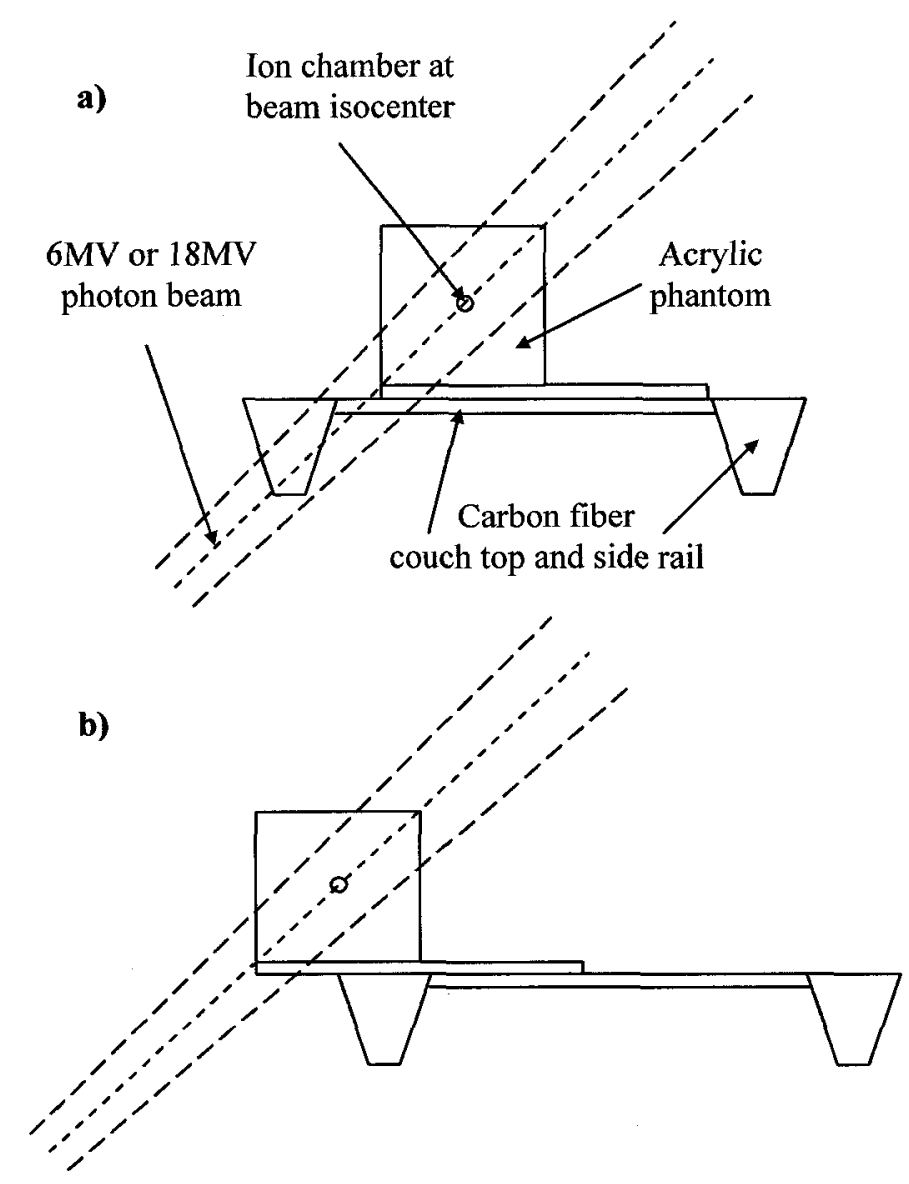

Figure 4.1. The experimental geometry for in-phantom measurement of dose reduction, calculated by the ratio of readings (a) with and (b) without the couch. In-air measurements were made under identical geometry except the phantom is replaced with brass buildup caps. 


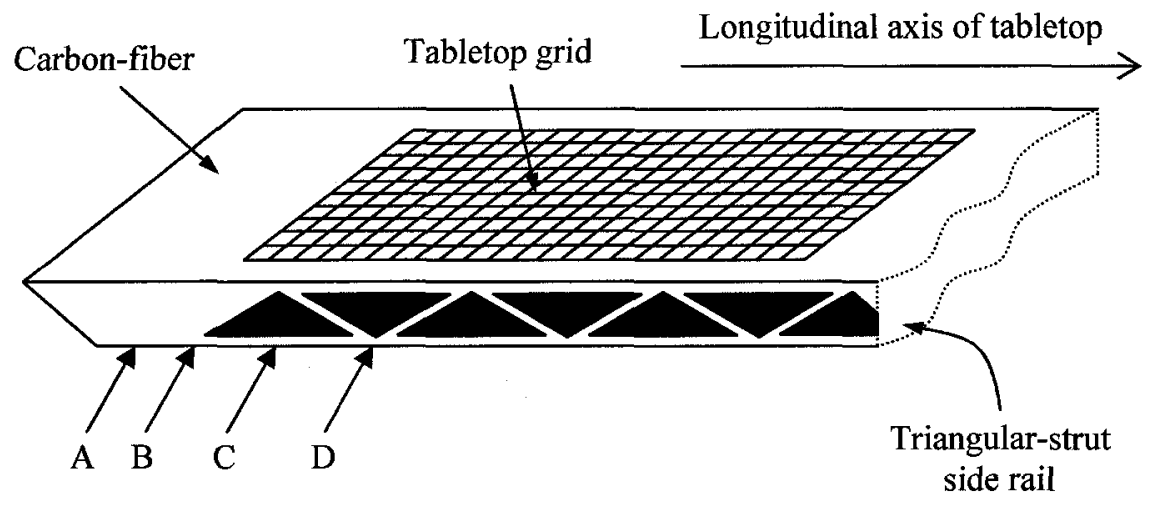

Figure 4.2. Longitudinal intersection of the beam with the treatment couch. At location A the entire field intersects the solid carbon fiber region of the couch. At locations B through D the beam transits the open strut region of the couch. 


\subsection{Results}

Attenuation of 6-MV and 18-MV photon beams by the carbon fiber side rails of the treatment tabletop was measured in-air for both $5 \times 5 \mathrm{~cm}^{2}$ and $10 \times 10 \mathrm{~cm}^{2}$ fields. The results are shown in Table 4.1. As expected, the attenuation is field size- and energydependent, the largest attenuation being $16.2 \%$ for smaller field sizes and lower energies. Attenuation is greatest near the head end of the tabletop (region A).

Measurements were also made in-phantom under the geometric conditions shown in Fig. 4.1(a) and (b). The effect of beam attenuation on the dose at depth in-phantom shows field size and energy dependence similar to the in-air observations. At the point of measurement, $100 \mathrm{~cm}$ source-to-axis distance, $14.1 \mathrm{~cm}$ depth, attenuation of the treatment field by the tabletop reduces the dose by up to $7.4 \%$ and is shown in the last column of Table 4.1.

To determine how well the planning system mitigates this error, comparison was made between the predictions of Theraplan Plus and measurements. The results are shown in Table 4.2. The first column under Dose Error compares measured and calculated dose when there is no couch in the beam, and represents how well our model in Theraplan Plus performs for this specific geometry. The second column compares the routine clinical situation where the table is physically present but ignored in planning. The final column shows the difference between measured and calculated dose when the tabletop is included in the Theraplan calculations. As shown, our model in Theraplan can 
Table 4.1. In-air and in-phantom measurements of the effect of beam attenuation by the Medtec clinical treatment tabletop. The estimated error for all measurements is $\pm 1 \%$.

\begin{tabular}{|c|c|c|c|c|}
\hline \multirow{2}{*}{ Energy } & \multirow{2}{*}{ Field size } & \multicolumn{2}{|c|}{$\begin{array}{l}\text { In-air beam fluence } \\
\text { attenuation (\%) }\end{array}$} & \multirow{2}{*}{$\begin{array}{c}\text { In-phantom } \\
\text { dose reduction } \\
(\%) \\
\text { Strut region }\end{array}$} \\
\hline & & Solid region & Strut region & \\
\hline \multirow{2}{*}{$6 \mathrm{MV}$} & $5 \times 5 \mathrm{~cm}^{2}$ & 16.2 & 5.8 & 7.4 \\
\hline & $10 \times 10 \mathrm{~cm}^{2}$ & 15.3 & 5.5 & 6.8 \\
\hline \multirow{2}{*}{$18 \mathrm{MV}$} & $5 \times 5 \mathrm{~cm}^{2}$ & 9.9 & 3.6 & 5.0 \\
\hline & $10 \times 10 \mathrm{~cm}^{2}$ & 9.3 & 3.5 & 4.7 \\
\hline
\end{tabular}

Table 4.2. The difference between measured and calculated (TPP - Theraplan Plus) dose for 6-MV and 18-MV fields with and without attenuation from the treatment tabletop.

\begin{tabular}{||c|c|c|c|c||}
\hline \multirow{2}{*}{ Energy } & \multirow{2}{*}{ Field size } & $\begin{array}{c}|c| \\
\text { Deasured w/o table } \\
\text { vs } \\
\text { TPP w/o table }\end{array}$ & $\begin{array}{c}\text { Measured w/ table } \\
\text { vs } \\
\text { TPP w/o table }\end{array}$ & $\begin{array}{c}\text { Measured w/ table } \\
\text { vs } \\
\text { TPP w/ table }\end{array}$ \\
\hline \hline \multirow{2}{*}{$6 \mathrm{MV}$} & $5 \times 5 \mathrm{~cm}^{2}$ & 3.0 & 10.4 & 1.4 \\
\cline { 2 - 5 } & $10 \times 10 \mathrm{~cm}^{2}$ & 2.3 & 9.1 & 1.3 \\
\hline \multirow{3}{*}{$18 \mathrm{MV}$} & $5 \times 5 \mathrm{~cm}^{2}$ & 3.1 & 8.1 & 1.3 \\
\cline { 2 - 6 } & $10 \times 10 \mathrm{~cm}^{2}$ & 2.3 & 7.0 & 0.4 \\
\hline
\end{tabular}



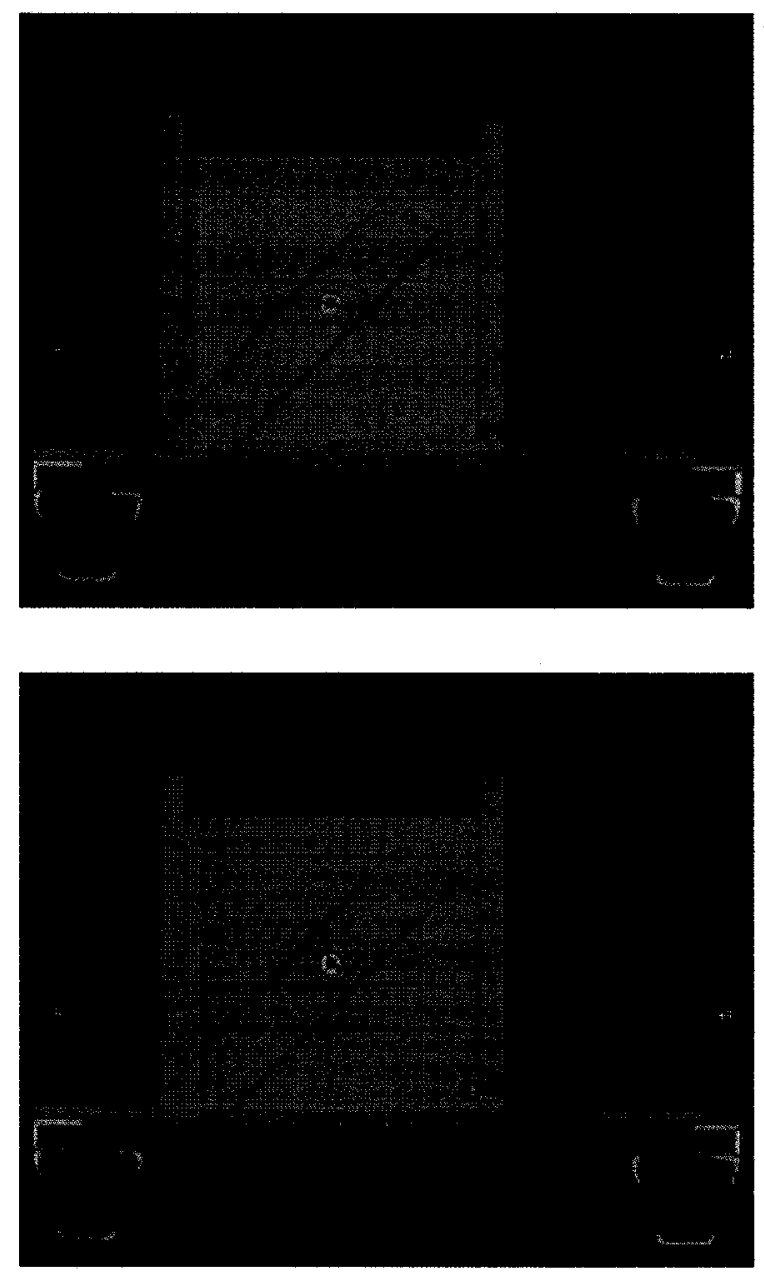

Figure 4.3. Theraplan isodose distributions calculated without (top) and with (bottom) the treatment couch included. 
only predict dose for the geometry in question to about $3 \%$. This is due to the complex geometry of the experiment combined with limitations in the pencil beam dose calculation algorithm. At the time of this study, this algorithm was used clinically at the Ottawa Hospital Cancer Centre. The treatment table introduces a dose error up to $7.4 \%$ at the point of interest. When we introduce the tabletop into the calculations, Theraplan overcompensates for the table, but the total error becomes less than $1.4 \%$. Figure 4.3 shows the Theraplan calculated dose distribution with and without the treatment couch.

\subsection{Discussion}

Carbon fiber materials are often considered to be effectively transparent to high-energy photons, particularly when compared to materials previously used for radiotherapy support structures, such as steel (Meara et al 1998). This is reflected in the fact that no general-purpose commercial treatment-planning system provides a mechanism to account for the treatment couch during the planning process. It should be noted that Tomotherapy (TomoTherapy Incorporated, Madison, WI) planning systems do provide such a capability. For beams passing through thin carbon fiber meshes and table inserts, the assumption of transparency may be clinically acceptable with the exception of surface dose effects as described by Carl and Vestergaard (2000).

Oblique treatment portals are commonly used (e.g., tangential breast, oblique lung, six-field prostate, and conformal brain), and, depending on the setup geometry, beams may pass through the side rails of the treatment couch. Under these conditions, dose reductions in-phantom of up to $7.4 \%$ were measured, while attenuation of up to 
$16.2 \%$ was measured for beams traversing the solid carbon fiber panel region at the superior end of the couch. If ignored, these are dose errors can be clinically significant. For example, an oblique opposed pair for a lung boost could easily have the posterior beam passing through the couch rails. In this case, for equally weighted beams, the total localized dose error would be $3.7 \%$ or greater, depending on the location of the patient on the couch.

It has been shown that a small change in the dose can result in a much larger change in the local response of the tissue. For example, Sanchez-Nieto and Nahum (1999) suggest that for prostate cancer a dose reduction of only $20 \%$ to $5 \%$ of the target volume can reduce the tumor control probability (TCP) by as much as $18 \%$. Mijnheer et al (1987) concluded that an overall accuracy of $\pm 3.5 \%$ in the dose delivered to the ICRU reference point is required. Clearly, the localized dose error arising from ignoring couch attenuation exceeds the recommended dose uncertainty and may result in significant reductions in TCP. The assumption that carbon fiber is radiation transparent is not valid, and ignoring the attenuation can be clinically significant.

Mitigation of attenuation by beam avoidance is difficult, particularly with indexed couches and immobilization devices. One solution is to include the couch in the dose calculation, although this is not straightforward due to the structural differences between the CT couch and the LINAC couch. This study has shown that the Theraplan Plus planning system can predict the effect of the treatment couch on the dose distribution to better than $3 \%$. 
We are currently exploring methods to routinely merge CT images of the treatment tabletop with patient CT scans. This involves replacing the portion of the image containing the CT couch with a previously acquired image of the treatment tabletop while maintaining the integrity of the patient data. The spatial relationship between the couch and the patient must be invariant between planning and treatment for this approach to be robust. This requirement is true for the lateral (IEC X) direction and to a lesser extent for the longitudinal (IEC Y) direction. Clearly, left-to-right shifts in patient position will result in the beam intersecting different components of the treatment couch with varying attenuations. Thus, the approach will require stringent patient indexing left to right. The variation in attenuation moving longitudinally along the couch has been explored. The results are shown in Fig. 4.4. It can be seen that the attenuation varies by $\pm 1.5 \%$ along the entire region investigated, but by only $\pm 0.5 \%$ over the portion of the couch defined by the tabletop grid. Thus, the requirement for indexing longitudinally is less stringent than left to right.

Radiotherapy techniques such as intensity-modulated radiotherapy use smaller field sizes and a variety of gantry angles, increasing the need to address this clinical problem. Routine consideration of couch attenuation requires that patients be positioned reproducibly by methods such as indexing. 


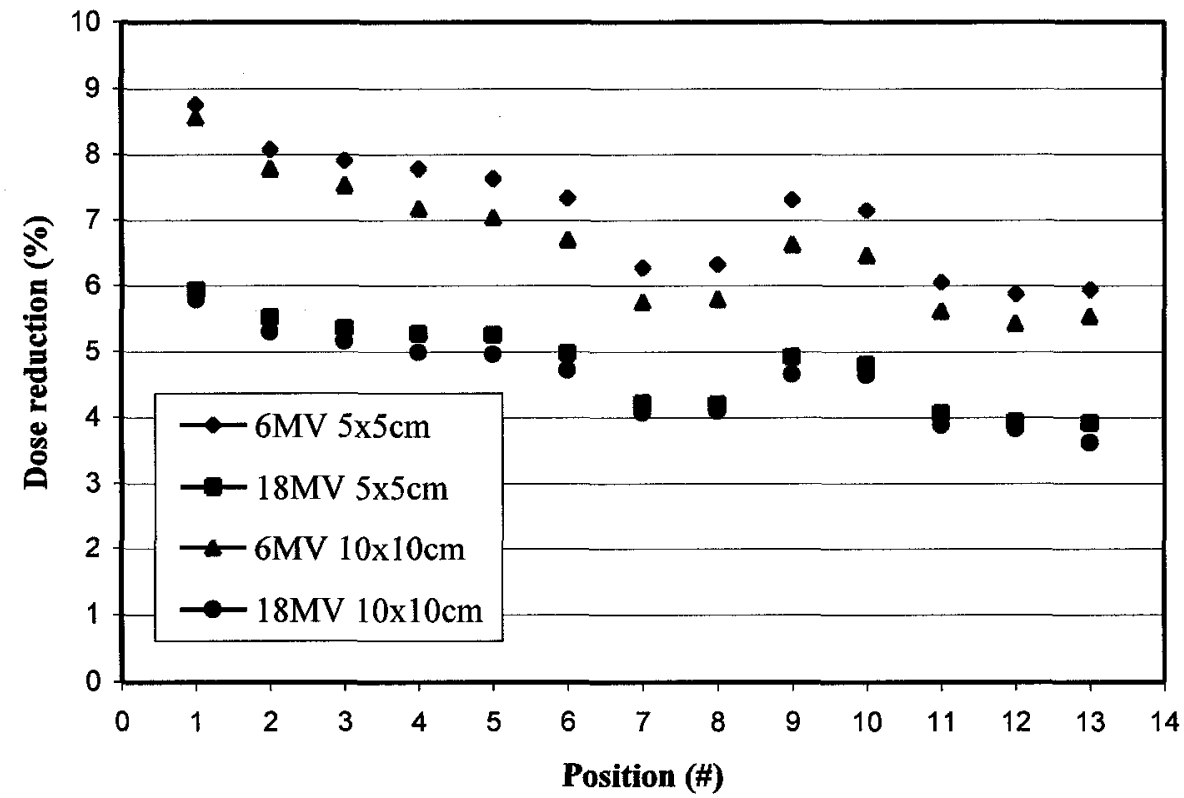

Figure 4.4 The dose reduction from attenuation by the tabletop at various positions along its length. The estimated error for all measurements is $\pm 1 \%$. 


\subsection{Conclusions}

This study investigated the dose error resulting from ignoring the beam attenuation by a carbon fiber treatment couch. It was found that neglecting this attenuation could result in clinically significant errors and that by including the treatment couch as part of the CT planning dataset, this error could be reduced significantly, even by a relatively simple dose calculation algorithm (pencil beam). We propose routine incorporation of the treatment couch as part of the treatment-planning dataset and have determined that this would require patient immobilization and indexing of the immobilization device with respect to the treatment tabletop. 


\section{Chapter 5}

\section{Treatment planning dose errors in the presence of hip and spinal prostheses}

\subsection{Introduction}

In external beam radiation therapy (EBRT), treatment planning dose calculations are usually based on patient images taken with a kilovoltage CT. Treatment planning systems (TPSs) convert the CT data (Hounsfield Units) to relative electron densities (or mass density depending on the planning system), which are subsequently used by the TPS algorithm to calculate dose. However in the presence of high atomic number materials, such as hip prostheses and spinal rods, two main sources of error can limit the accuracy of the TPS dose calculation. Firstly, high- $Z$ materials create problems for the CT reconstruction algorithm resulting in streak artifacts in the CT images. Consequently, an incorrect Hounsfield number can be assigned to voxels containing normal tissue. The 
artifacts also prevent accurate delineation of the tumour volume, critical anatomy and even the high- $Z$ prosthesis itself. The second source of error is an incorrect mapping of the Hounsfield number to relative electron density for high- $Z$ materials. This will be discussed in further detail below. Both of these errors can be reduced with the use of megavoltage CT (MVCT) images rather than standard kilovoltage (kVCT) images. This study will examine the dose error resulting from propagating the two sources (artifacts and electron density mapping) of error through the treatment planning system and compare kVCT and MVCT-based treatment planning calculations in the presence of high-Z prosthetic devices.

The mapping of kVCT Hounsfield units to electron density depends on the type of photon interactions occurring at kilovoltage energies. In terms of the relative contribution of photon interaction processes in water, photo-electric absorption and Compton scatter are approximately equal at around $25 \mathrm{keV}$ (Johns and Cunningham 1983). Between $60 \mathrm{keV}$ and $200 \mathrm{keV}$, both the photo-electric and Compton processes contribute to the total energy absorption. Between $200 \mathrm{keV}$ and $2 \mathrm{MeV}$, the Compton process dominates. Pair production contributes for photons with energies above 1.02 $\mathrm{MeV}$ but really only becomes important for photons above $5 \mathrm{MeV}$. In addition, the Compton mass-attenuation coefficient is independent of $Z$ (atomic number) because the photon interaction is essentially with a free or stationary electron. However, the massattenuation coefficient for the photoelectric effect is proportional to $Z^{3}$ (Evans, 1955). Thus for conventional CT scanners that use photons in the kilovoltage range, the main interaction process is primarily Compton scatter in soft tissue but there is an increased 
amount of photoelectric interaction in bones and for higher $\mathrm{Z}$ materials the photoelectric process can dominate (Attix 1986, McCollough and Zink 1999). Consequently, the Hounsfield numbers are a function of both the electron density and the atomic number of the material. The implication of this dual dependency is that various materials with different atomic numbers but equal electron density can be represented by the same Hounsfield unit. Hence, the conversion from Hounsfield units to electron density is nonunique.

The use of MVCT as an alternative to conventional $\mathrm{kVCT}$ is becoming increasingly available clinically (Aubin et al 2006, Morin et al 2007). At mega-voltage energies, the Compton interaction cross section remains nearly independent of $\mathrm{Z}$ and the photoelectric interaction coefficient is negligible, but the linear attenuation coefficient for pair production is proportional to $\mathrm{Z}$. When the TomoTherapy Hi*Art II system (TomoTherapy, Inc., Madison, WI, USA) is used for imaging, the nominal energy of the incident electron beam is reduced from $6 \mathrm{MV}$ to $3.5 \mathrm{MV}$ (Jeraj et al 2004). In this energy range, the interaction cross section contribution of the pair production process is minimal and it does not play a significant role in TomoTherapy MVCT imaging. As a result, the relationship between the MVCT number and electron density is expected to be linear, providing a more reliable map than Hounsfield numbers (Ruchala et al 2000, Simpson et al 1982).

Artifacts caused by high $-Z$ materials introduce two problems. They reduce the diagnostic clarity required for tumour delineation and they result in incorrect electron 
density values used in the dose calculation. Attempts have been made to remedy these errors but none have established themselves into routine clinical procedure (Kachelriess et al 2001, Morin and Raeside 1982, Robertson et al 1997). Many of these methods such as the iterative de-blurring technique showed promise in simple phantoms but were less successful with complex phantoms and patient scans. Langen et al (2005) showed that MVCT images from a TomoTherapy system could be used for treatment planning dose recalculation and verification. The MVCT- and kVCT-based target dose computations and dose-volume histograms (DVHs) were shown to agree within $0.5 \%$.

\subsubsection{MVCT image quality}

In normal soft tissues MVCT images will be inferior to kVCT images, but in specific cases such as in the presence of high- $Z$ prostheses, the benefits can out-weigh these deficiencies. This section examines some of the pros and cons of MVCT images for diagnostic and therapeutic use. The ability of an x-ray imaging system to differentiate between materials (contrast) is mainly due to differences in attenuation coefficients at a given energy. Thus the soft tissue contrast will inherently be better at $\mathrm{kV}$ energies due to the increased amount of photo-electric interaction. As previously mentioned, for megavoltage photons the attenuation coefficient is proportional to the electron density and is nearly independent of $Z$. Therefore, the object contrast will be relatively constant over a wide range of megavoltage energies. Additionally, prosthetic materials such as titanium have a significantly greater density than soft tissue and will generally have sufficiently high contrast at MV energies (Ruchala et al 1999). 
Reducing the number of MV photons will reduce the dose at the cost of decreased signal-to-noise ratio (SNR) and detection of soft-tissue contrast. However, the greater penetration of MV photons relative to $\mathrm{kV}$ photons results in a larger fraction of the MV photons traveling through the patient and reaching the detector. Conversely, this increased efficiency is reduced by photons that not only reach the detector but penetrate through it undetected. The TomoTherapy system utilizes a conventional xenon CT detector with the xenon maintained at 5 -atm pressure. These detectors are only $\sim 1 \%$ efficient at MV energies. Standard solid state $\mathrm{kVCT}$ detectors are over $80 \%$ efficient at $\mathrm{kV}$ energies and over $20 \%$ efficient at MV energies. Unfortunately, solid state detectors are costly and prone to radiation damage.

Groh et al (2002) studied the SNR as a function of $\mathrm{x}$-ray energy between $\mathrm{kV}$ and MV energies for cone-beam CT (CBCT). Their results showed that the $\mathrm{kV} \mathrm{CBCT}$ performed better than the MV CBCT in terms of SNR versus dose. $\mathrm{kV} \mathrm{CBCT}$ imagers can provide soft-tissue visibility ( $0.5 \%$ contrast for objects with $5 \mathrm{~mm}$ diameter) at doses as low as a few cGy. The low detection efficiency of the MV CBCT imagers increased the dose by a factor between 10 and 100 to achieve comparable soft tissue characterization as the $\mathrm{kV}$ CBCT images. In 2003, Ruchala et al showed that a TomoTherapy imaging system could achieve $1 \%$ and $3 \%$ contrast visibilities with a 0.9 cGy and 7 cGy MVCT images, respectively. In 2005, Meeks et al characterized the performance of the MVCT imaging system on a TomoTherapy unit. The MVCT noise standard deviation $(2 \%-4 \%)$ was found to be approximately twice as large as in a conventional CT simulator. A subjective test of image resolution performed by scanning 
a high resolution hole pattern revealed that the visible resolution was approximately 1.25 $\mathrm{mm}$ for a $512 \times 512$ pixel image.

\subsubsection{Aim of study}

The aim of this research was to assess the reduction in treatment planning dose calculation errors resulting from the use of MVCT images as opposed to standard kVCT images. The treatment planning dose calculations based on pencil beam and superposition algorithms were performed on $\mathrm{kVCT}$ and MVCT images of two wax phantoms embedded with high- $Z$ prosthetic devices. Dose calculations performed by the EGSnrc/DOSXYZnrc system for particle transport were used as a gold standard.

\subsection{Materials and Methods}

\subsubsection{Treatment planning dose calculations}

All dose measurements were performed on a Siemens Mevatron linear accelerator at the Ottawa Hospital Regional Cancer Centre. Measurements and treatment planning dose calculations were performed on two wax phantoms embedded with the titanium alloy prosthetic devices as shown in Figure 5.1. The first phantom contained two different hip prostheses (AACE 32, product number 6976-1 and ALYDB 6, product number 6280-6010) while the second phantom contained a spinal $\operatorname{rod}\left(\right.$ Monarch ${ }^{\circledR}$ spinal system, DePuy AcroMed, Raynham, Massachusetts, USA) lying between two foam blocks representing lung lobes. Figure 5.2 shows a cross-sectional slice through the hip and spine prosthesis phantoms, illustrating the placement of the prosthetic devices and foam blocks in the wax. Ion chamber dose verification measurements were taken near the isocenter of the 
phantoms at a depth of $6.8 \mathrm{~cm}$. The ion chamber holes were plugged with tissue equivalent material when not in use.

Standard hip prostheses are usually comprised of an acetabular cup and a femoral component consisting of a stem and a head. In the first hip prosthesis, the femoral stem was used with a partially hollowed head, but only the femoral stem was utilized in the second hip prosthesis. The physical density of the hip prostheses was $4.3 \mathrm{~g} / \mathrm{cm}^{3}$ and its elemental composition is listed in Table 5.1. The diameter of the spinal rod was $4.50 \mathrm{~mm}$ with a physical density of $4.43 \mathrm{~g} / \mathrm{cm}^{3}$. The relative composition of the rod follows the ASTM (American Standard for Testing Materials, West Conshohocken, PA, USA) F-136 specifications and is also shown in Table 5.1. Ion chamber holes were bored into each phantom to enable dose verification at a depth of $6.8 \mathrm{~cm}(100 \mathrm{~cm}$ SAD) with a NRC calibrated NE $25710.6 \mathrm{~cm}^{3}$ ion chamber calibrated with an NE 2570 electrometer. 
a)

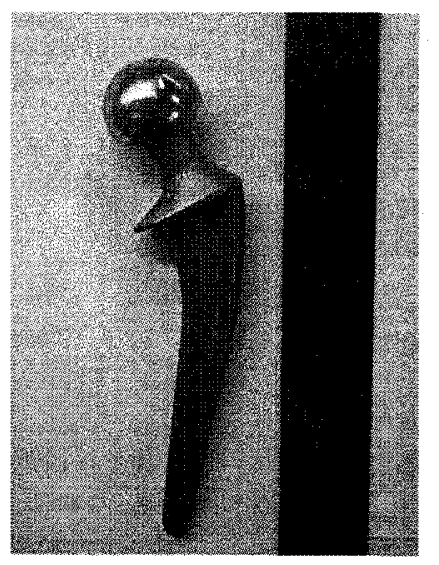

b)

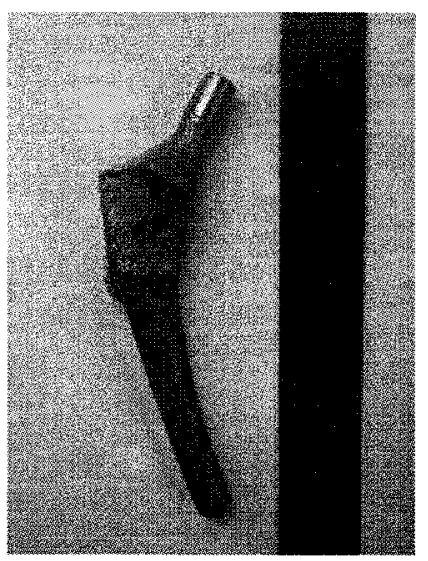

c)

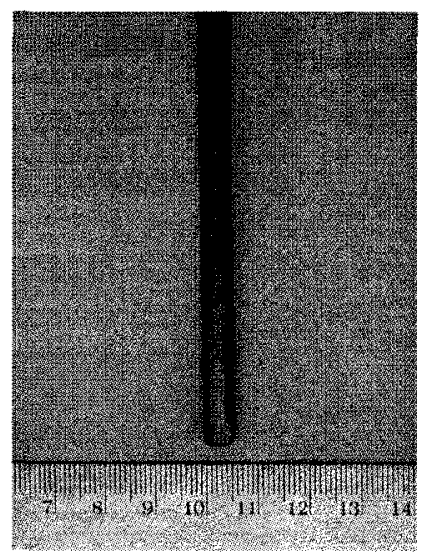

Figure 5.1 The prosthetic devices used in this study: a) hip prosthesis \#1 consisting of a femoral stem and head, b) hip prosthesis \#2 consisting only of the femoral stem and c) the spinal rod prosthesis. 
a)

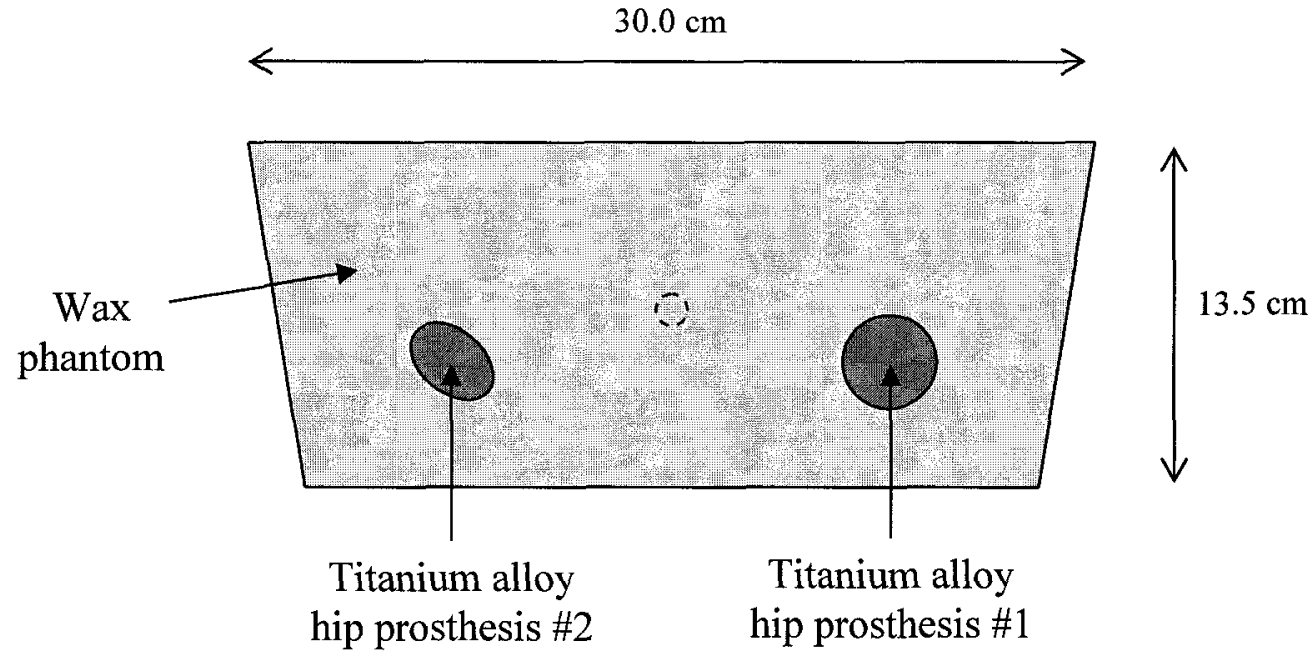

b)

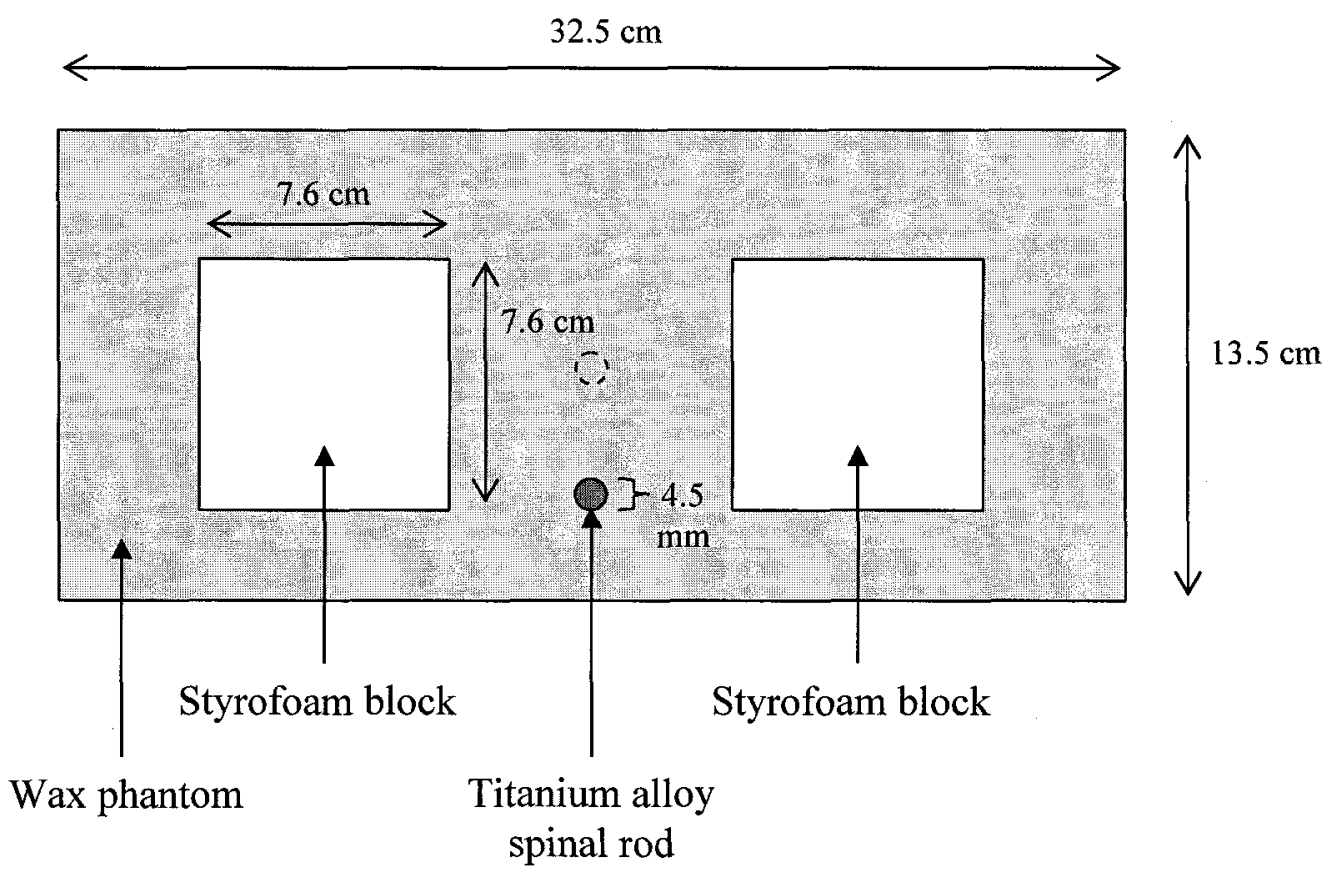

Figure 5.2 Diagrams illustrating cross-sectional slices of the a) hip prostheses phantom and b) spinal rod phantom. Ion chamber measurements were taken at a depth of $6.8 \mathrm{~cm}$ (marked by 5 ) and the hole was plugged during CT imaging. 
Table 5.1 Elemental composition of hip and spinal prosthetic devices used in this study.

\begin{tabular}{ccc}
\hline \multirow{2}{*}{ Element } & \multicolumn{2}{c}{$\%$ Weight } \\
\cline { 2 - 3 } & Hip Prostheses & Spinal Rod \\
\hline Hydrogen & --- & $0.015 \mathrm{max}$ \\
Nitrogen & $0.08 \mathrm{max}$ & $0.05 \mathrm{max}$ \\
Carbon & $0.13 \mathrm{max}$ & $0.08 \mathrm{max}$ \\
Oxygen & $0.25 \mathrm{max}$ & $0.13 \mathrm{max}$ \\
Iron & 3.5 to 4.5 & $0.25 \mathrm{max}$ \\
Vanadium & 5.6 to 6.5 & 3.5 to 4.5 \\
Aluminum & Balance & 5.5 to 6.5 \\
Titanium & & Balance \\
\hline
\end{tabular}


The kilo-voltage and -mega-voltage CT datasets ( $3 \mathrm{~mm}$ slice thickness with $3 \mathrm{~mm}$ spacing) of the entire phantoms were acquired on a Philips AcQsim large bore, single slice CT simulator (Philips Medical Systems, ON, Canada) and Tomotherapy HI-ART II imager (TomoTherapy Incorporated, Madison, WI), respectively. The image dataset from the CT simulator was taken with a $512 \times 512$ matrix resolution, $50 \mathrm{~cm}$ diameter field-of-view (FOV), $0.97656 \mathrm{~cm}$ pixel size and a nominal beam energy of $120 \mathrm{kV}$. MVCT images from HI-ART II unit were also taken with a $512 \times 512$ matrix resolution but the FOV was limited to $39 \mathrm{~cm}$ and a $0.75401 \mathrm{~cm}$ pixel size. The MVCT nominal beam energy was $3.5 \mathrm{MV}$.

Figures 5.3 and 5.4 show sample slices from the kVCT and MVCT datasets of the hip and spinal prosthesis phantoms, respectively. The abnormal shape of the phantoms reflects the shape of the wax molding. Note the difference in image artifacts between the $\mathrm{kV}$ and MV images. Figure 5.5 shows cross-sectional kVCT and MVCT slices of the two phantoms and the three different beam geometries examined in this study. In the first geometry, the beam is centered on the central axis of the hip phantom allowing us to examine the dose calculation errors caused by image artifacts in normal tissue arising from the distant prostheses. The second and third geometries focused on the region in the immediate vicinity of the hip prosthesis and the spinal rod, respectively. Treatment planning dose calculations were performed on both the $\mathrm{kV}$ - and MVCT datasets for $5 \times 5$ $\mathrm{cm}^{2}$ and $10 \times 10 \mathrm{~cm}^{2}$ field sizes at both $6 \mathrm{MV}$ and $18 \mathrm{MV}$ photon energies delivering 100 monitor units (100 cm SAD). The dose calculations were modeled by Theraplan Plus version 3.8 (pencil beam algorithm) and CMS (multi-grid superposition algorithm). A 
general overview of the convolution/superposition dose calculation algorithms was presented in Chapter 3.2, with specific reference to the collapsed-cone convolution, multi-grid superposition and pencil-beam techniques. The treatment planning systems map the CT data (Hounsfield units) to electron density relative to water (RED) using the data shown in Figure 5.6. The kVCT data for this table is listed in Table 3.1. This data was obtained by scanning a Gammex RMI electron density CT phantom RMI 467, serial \# 802428-1035 (Gammex RMI, Middleton, WI, USA) containing a wide range of materials with known electron densities and measuring the $\mathrm{CT}$ data values corresponding to each electron density.

Data for the MVCT numbers is shown in Table 5.2 along with values for mass density and relative electron density. The discrepancy between mass density and relative electron density introduces another form of dose error within the treatment planning calculations. The extent of this discrepancy is illustrated in Figure 5.7. One of the main assumptions made by most clinical TPSs is that the human body consists of water of variable density. Hence, the dose predicted by these TPSs is presented as dose to water. This has been shown to be a reasonable approximation for compounds with densities less than that of water (as shown in Figure 5.7). However, for denser materials such as titanium, the discrepancy between mass density and RED can be significant (16\%). Since the dose is defined as "energy deposited per unit mass", the mass density of the target material must be known for an accurate dose calculation. Most clinical TPSs assume that the mass density scales with electron density, i.e. they assume that below a density of 1 all materials are water of lower density and above 1 they are a mixture of 
bone and water. Thus, there is a known systematic error in TP calculations that is dependent on the density of the target material. As was mentioned above, the largest discrepancies exist for high-Z materials such as those used for prosthetic devices or for filling dental cavities. Fortunately for radiotherapy patients with such implements, physicians are mainly concerned with the dose to normal tissue or a tumour rather than the dose to the high- $Z$ device.

It should also be noted that the range of MVCT values extends far beyond that of the HUs measured with the CT simulator. Near the maximum range of the KVCT scale, the HUs were measured for relatively dense materials such as aluminum $(\operatorname{RED}=3.12$, stainless steel $(\mathrm{RED}=6.56)$, brass $(\mathrm{RED}=6.78)$, copper $(\mathrm{RED}=7.35)$ and lead $(\mathrm{RED}=$ 10.78). With the exception of aluminum, all of these materials gave the same HU, just below 3000 . Thus the HU saturation point is reached for materials with REDs between 3.12 (aluminum) and 6.56 (stainless steel). 
a)

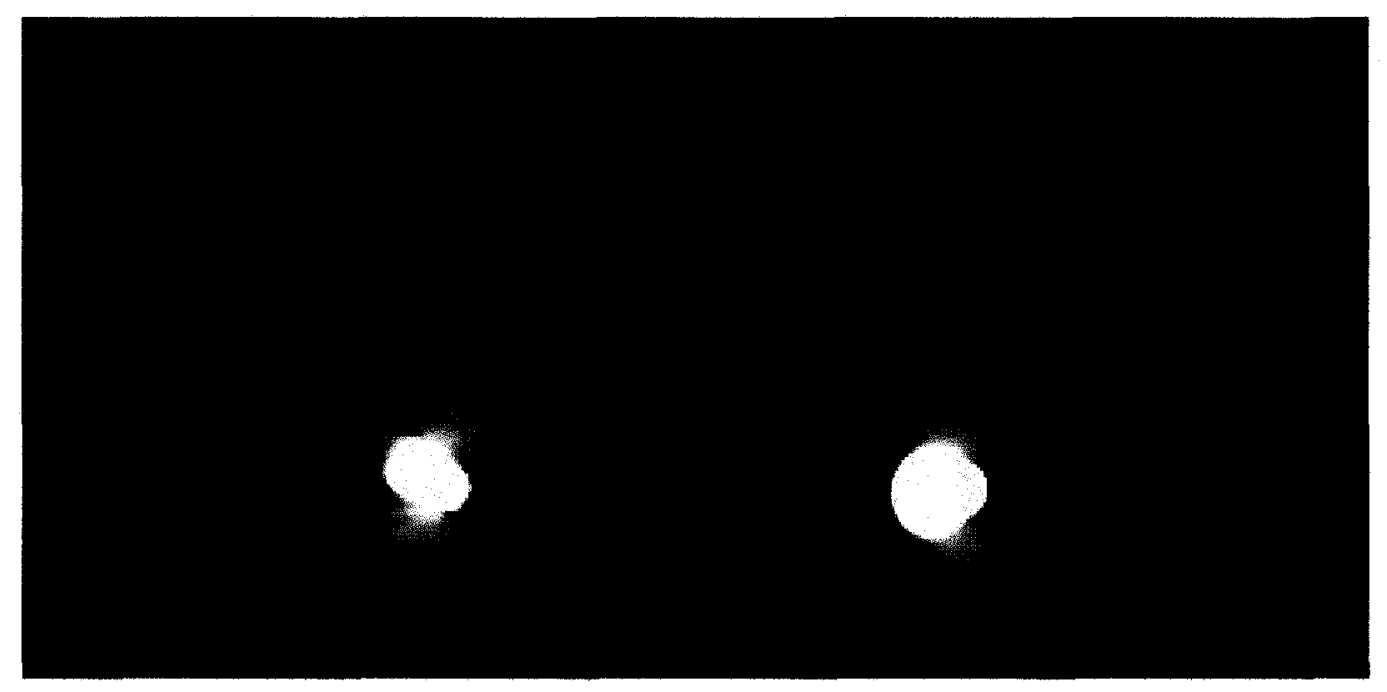

b)

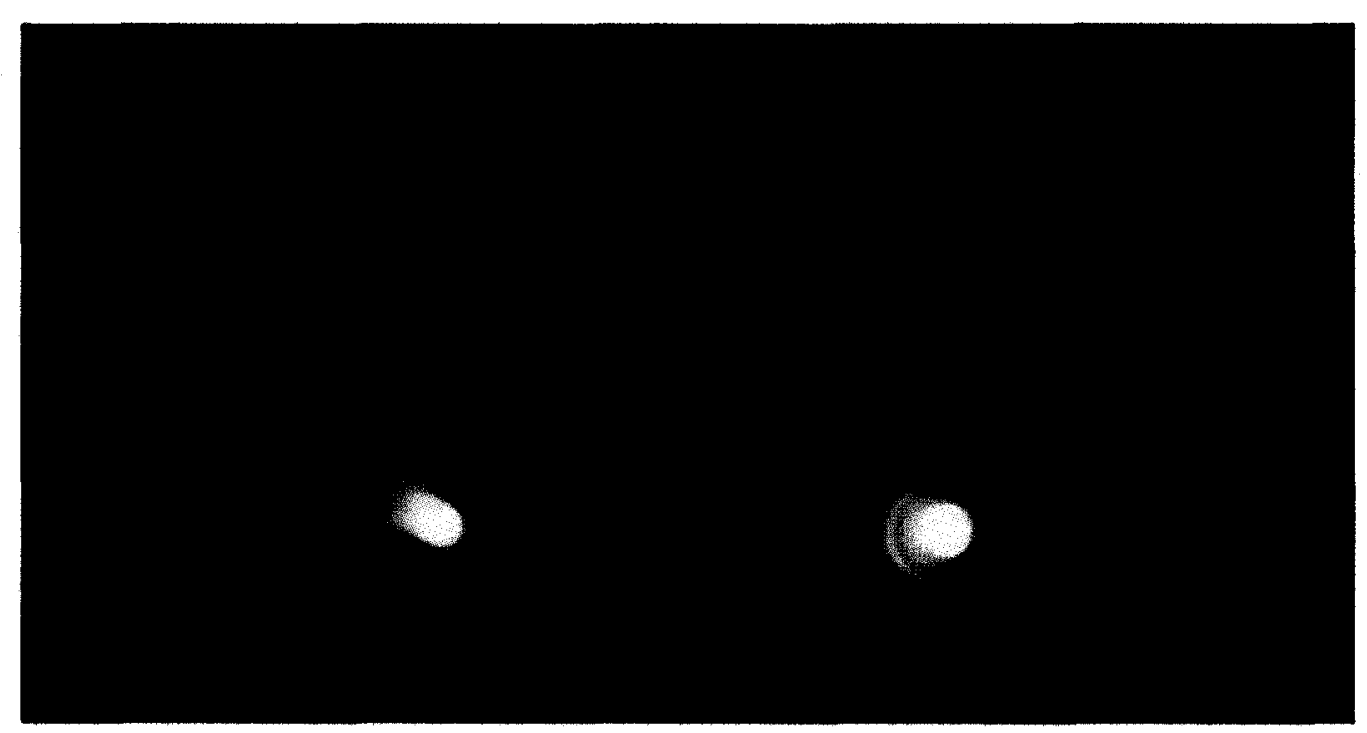

Figure 5.3 kVCT (a) and MVCT (b) images of the wax phantom containing the hip prostheses showing the differences in the artifacts in the two image types. 
a)

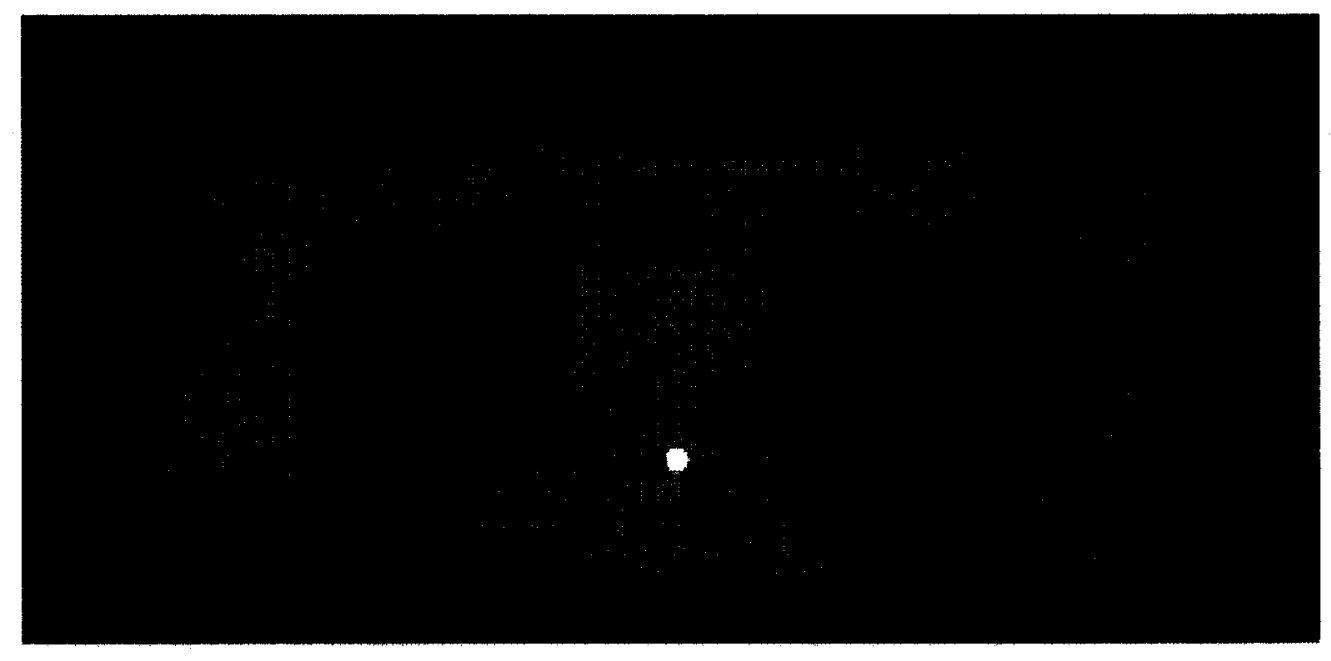

b)

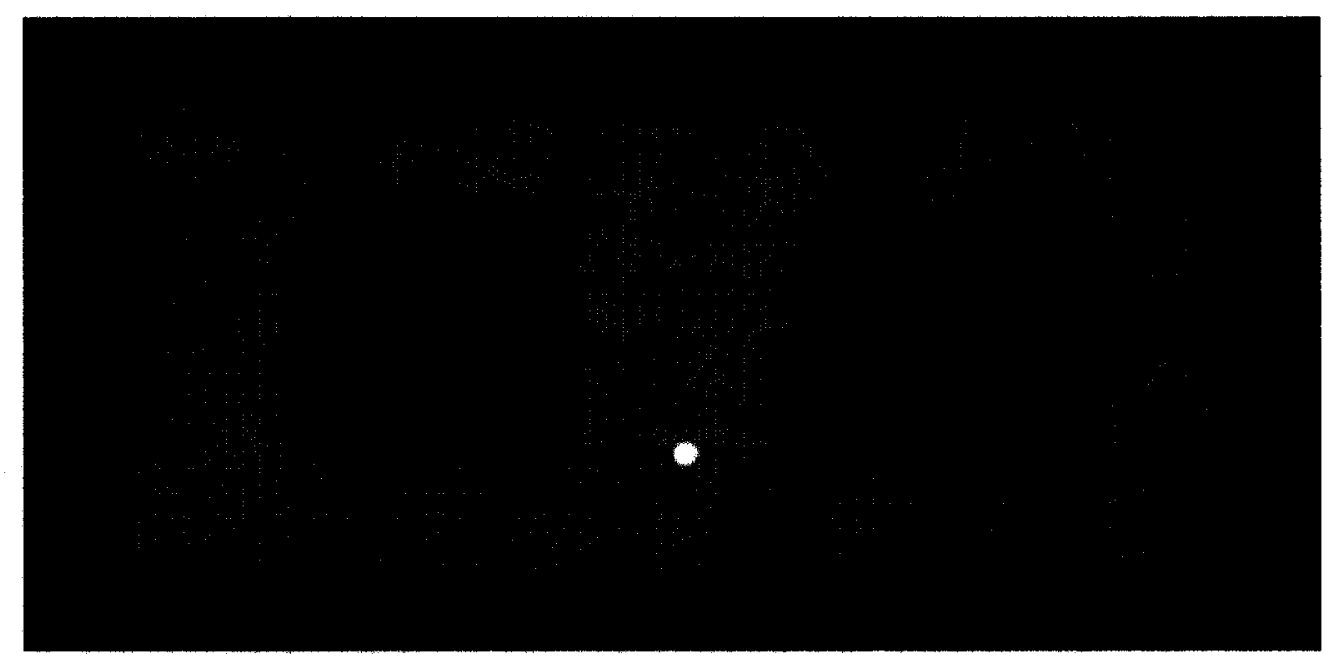

Figure 5.4 kVCT (a) and MVCT (b) images of the wax phantom containing the spinal prostheses showing the differences in the artifacts in the two image types. 
a)

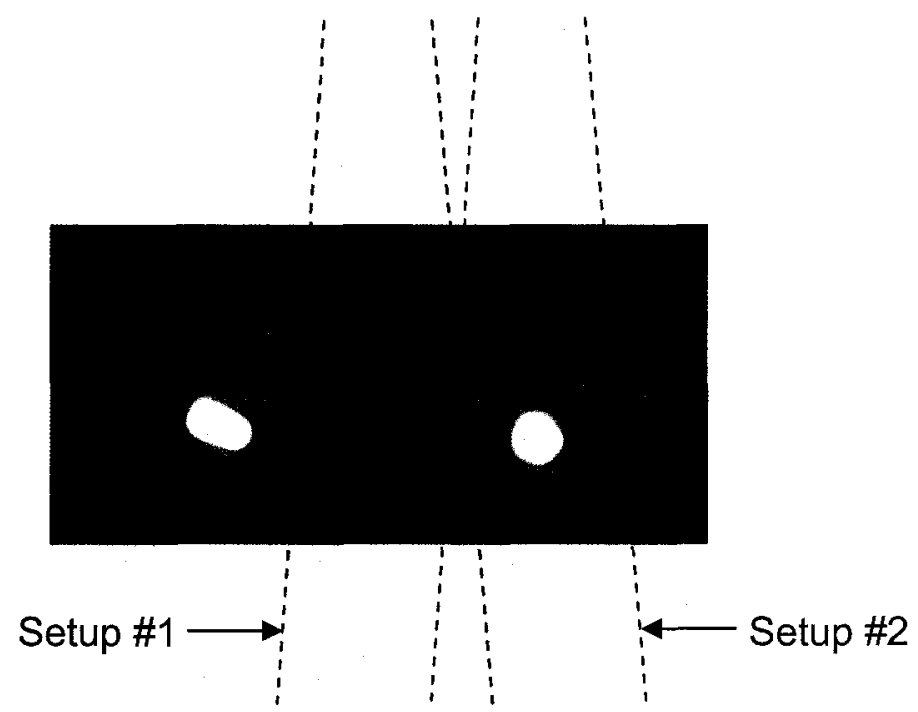

b)

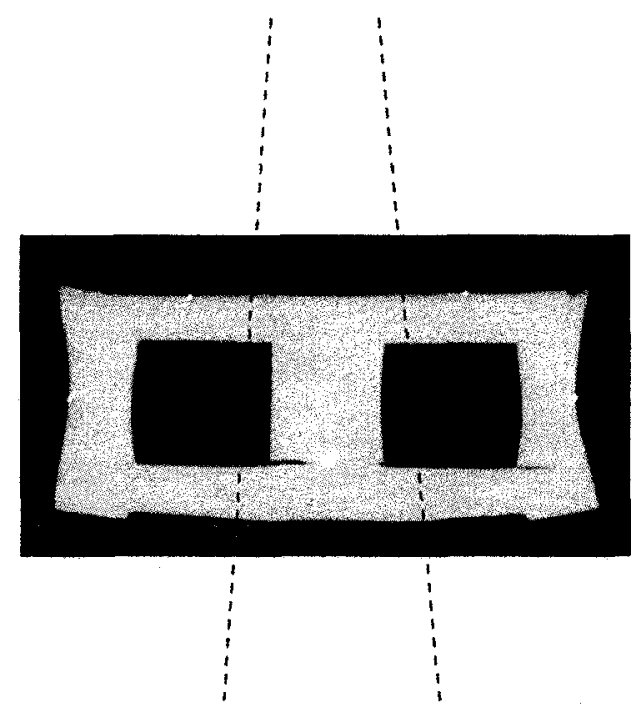

Setup \#3

Figure 5.5 Cross-sectional MVCT images depicting the beam geometries examined with the a) hip prostheses and b) spinal prosthesis phantoms. Differences in intensity between the two images are due to varying window and level settings. 


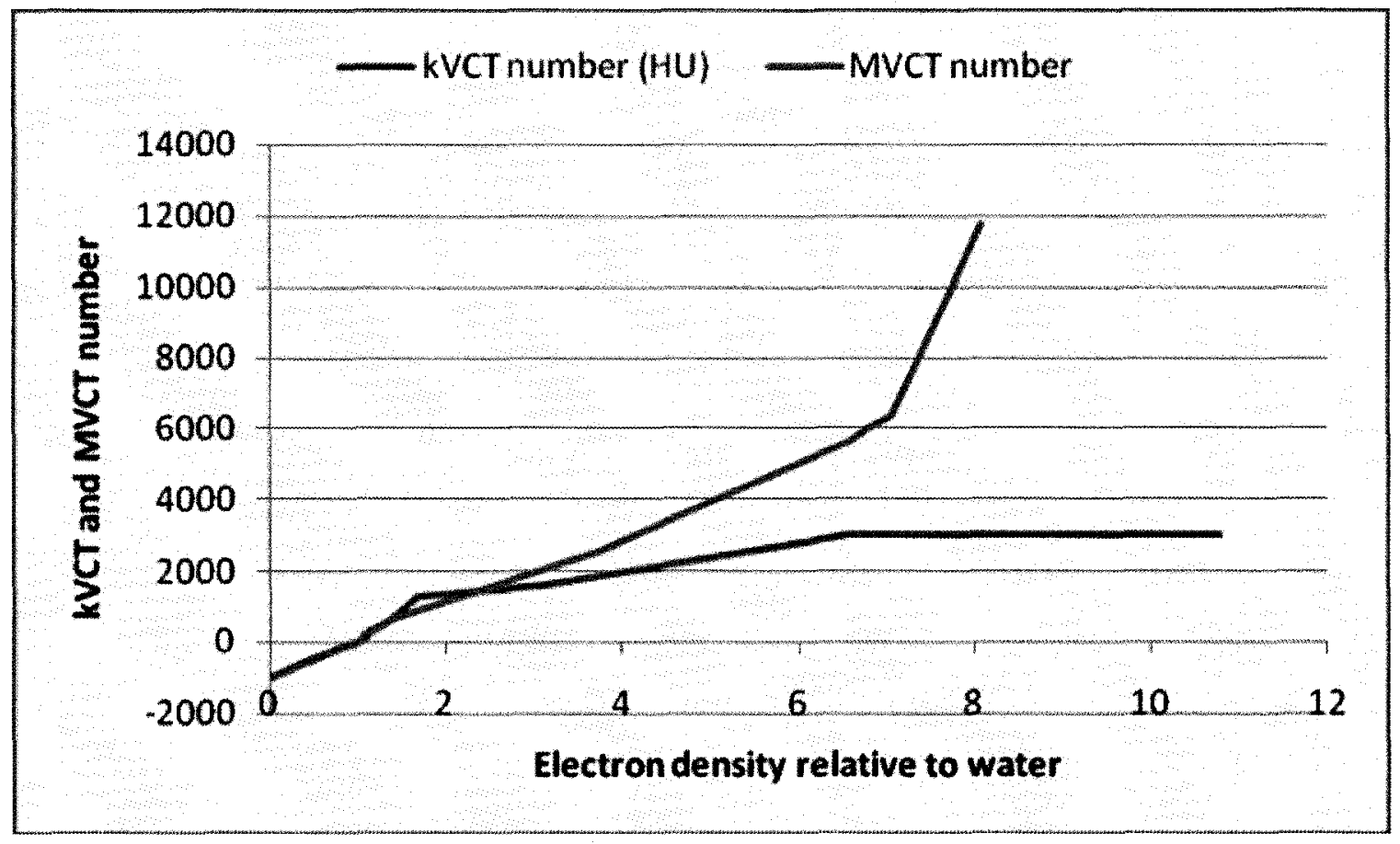

Figure 5.6 Relative electron density curves for both the kVCT and MVCT imagers, comparing kVCT (HU) and MVCT numbers. 
Table 5.2 A comparison of the mass density, electron density relative to water (RED) and MVCT Hounsfield unit (HU) data of various compounds.

\begin{tabular}{cccc}
\hline Material & $\frac{\text { Mass density }}{\left(\mathbf{g} / \mathbf{c m}^{3}\right)}$ & $\underline{\text { RED }}$ & $\underline{\text { HU }}$ \\
\hline air & 0.001 & 0.001 & -1000 \\
lung (LN-300) & 0.296 & 0.288 & -700.5 \\
lung (LN-450) & 0.472 & 0.453 & -513.1 \\
adipose & 0.950 & 0.925 & -34.00 \\
true water & 1.000 & 1.000 & 0.000 \\
brain & 1.053 & 1.049 & 84.55 \\
inner bone & 1.140 & 1.093 & 142.7 \\
pmma & 1.186 & 1.140 & 323.6 \\
cortical bone & 1.562 & 1.472 & 734.1 \\
titanium & 4.430 & 3.740 & 2568 \\
brass & 7.870 & 6.590 & 5624 \\
iron & 8.300 & 6.800 & 5995 \\
lead & 8.600 & 7.059 & 6366 \\
\hline
\end{tabular}




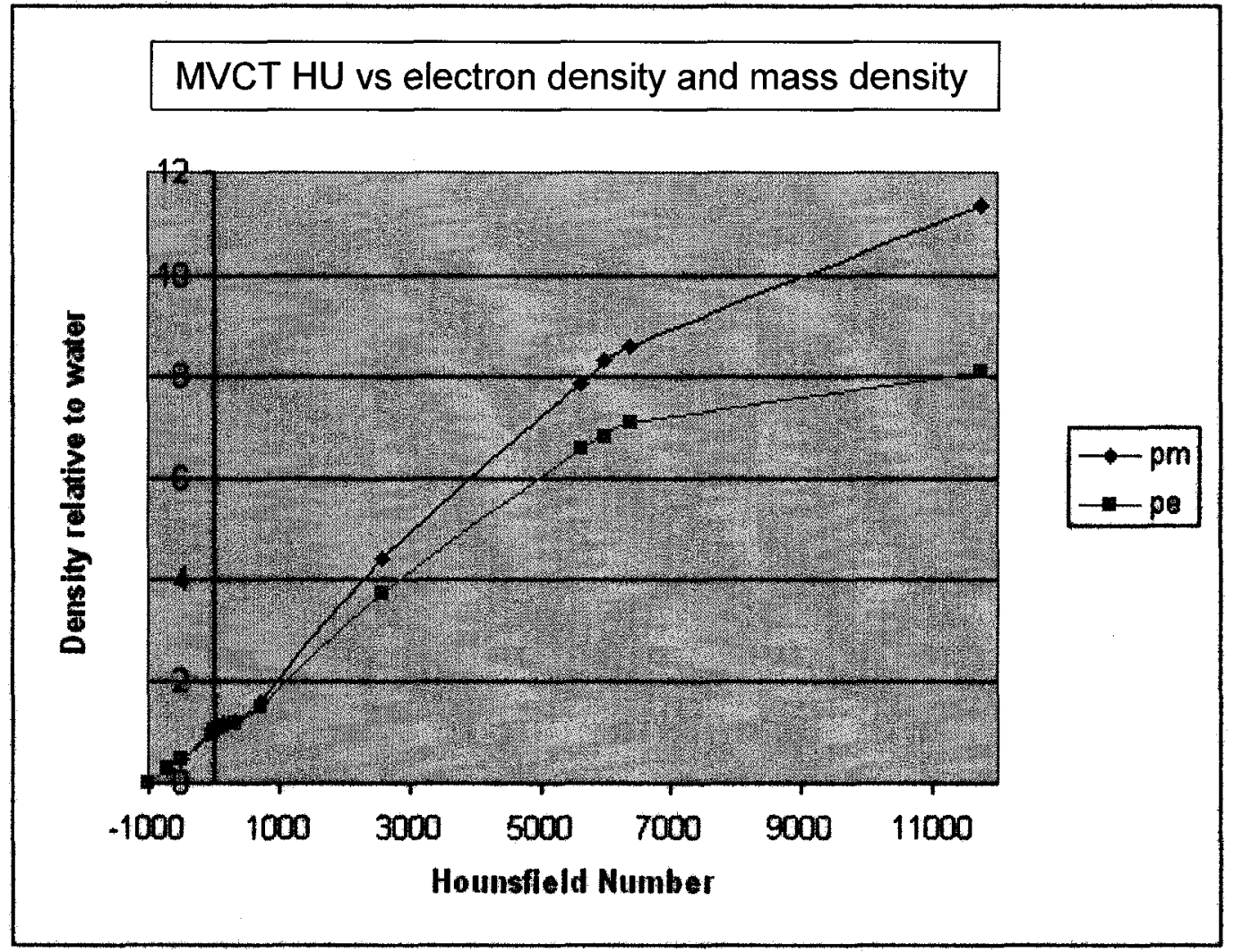

Figure 5.7 The mass and the electron density relative to water plotted as a function of the MVCT Hounsfield units. $p_{m}$ and $p_{e}$ represent the mass and electron densities, respectively. 


\subsubsection{Monte Carlo simulation parameters}

The Monte Carlo dose calculations were performed with the BEAMnrc/DOSXYZnrc system, based on the EGSnrc particle transport code (Kawrakow and Rogers 2002). The parameters of the beam model were determined from an extensive iterative process performed until the depth dose and profiles of the beams adequately replicated the linear accelerator commissioning data. Verification of the MC beam model is discussed in detail in Chapter 2 but this section will outline some important parameters.

The manufacturer provided all physical specifications of the linear accelerator beam head. In our model the 6-MV and 18-MV photon beams were generated using initial electron energy spectra with peak energies of 6.88 and $14.55 \mathrm{MeV}$, respectively, with asymmetrical Gaussian energy distributions (Sheikh-Bagheri and Rogers 2002) These electron beams were incident on the tungsten target with Gaussian spatial distributions and characterized with a FWHM of $0.5 \mathrm{~mm}$.

$\mathrm{AP}$ and $\mathrm{AE}$ are $\mathrm{MC}$ transport parameters that represent the low-energy thresholds for the production of secondary Bremsstrahlung photons and knock-on electrons, respectively. As mentioned in chapter 2, PCUT and ECUT represent the low energy threshold for which particle transport is cutoff and all remaining energy is deposited locally. For our simulations, these parameters were set at $\mathrm{AP}=\mathrm{PCUT}=0.010 \mathrm{MeV}$ and $\mathrm{AE}=\mathrm{PCUT}=0.700 \mathrm{MeV}$. Uniform Bremsstrahlung splitting was utilized as a variance reduction technique to increase photon production and the efficiency of our simulation. 


\subsubsection{Monte Carlo simulation phantom}

DOSXYZnrc was used in conjunction with CTcreate to simulate dose distributions in the hip and spine phantoms (Walters et al 2004). CTcreate is a stand-alone routine that reads in CT datasets and convert them into 3-dimensional virtual phantoms or patients. Thus EGSnrc could be used as a gold standard for our dose calculations as long as the phantoms were accurately represented by the CT datasets. For our purposes, the MVCT datasets were used as the basis for our MC simulations. These datasets showed fewer streak artifacts and greater prosthesis delineation than the kVCT datasets, and as previously mentioned, can be more accurately mapped to the electron density. Theoretically, DOSXYZnrc offers the tools to design a mathematical phantom with correct composition. However, the complex structure of the hip prostheses would prevent the accurate design of a virtual phantom.

For the purposes of generating reference dose distributions with MC, all artifacts in the wax region of the phantoms were removed from the MVCT images. This was accomplished by segmenting the DICOM files into raw image and patient data, then editing the raw image file. In each phantom, an average Hounsfield value was sampled from a wax region where no artifacts were present and the Hounsfield values in the artifact-filled wax regions were replaced with this average value. Thus, the end result was a completely homogeneous wax region in the CT datasets used for the MC simulation. No artifacts were removed from the hip prostheses due to their complex shape. This posed no problem for hip prosthesis \#2 since the femoral stem was completely solid, but it did result in a blurring of titanium-equivalent MVCT numbers 
into the hollow regions of hip prosthesis \#1. However, artifacts within the prostheses were already greatly reduced in the MVCT images over the KVCT images.

Figure 5.8 shows MVCT Hounsfield unit profiles through the hip prostheses before and after the artifacts were removed from the images. These profiles were taken from the cross-sectional MVCT images of Figure 5.9, both a) before and b) after artifact removal in the wax region. The depth of the profiles was selected so that significant portions of both prostheses would be included. The blue dashed line illustrated in Figure 5.9 specifies the depth of all profiles examined with the hip prostheses phantom. It can be seen that the artifacts in the original image in the region between the two prostheses reduced the MVCT number relative to the surrounding wax material. As expected, the MVCT number in the hip regions is identical in both profiles since this region was left untouched.

Figure 5.10 shows MVCT profiles through the spinal prosthesis before and after artifacts were removed from the wax region of the phantom. These profiles were taken from the cross-sectional MVCT images of Figure 5.11. The depth of the profile is illustrated by the blue dashed line in Figure 5.11. The red dashed line indicates the depth of profiles examined later in this chapter. As was done with the hip prostheses phantom, an average pixel value was homogeneously assigned to all wax regions while the Styrofoam and spinal rod regions were untouched. 


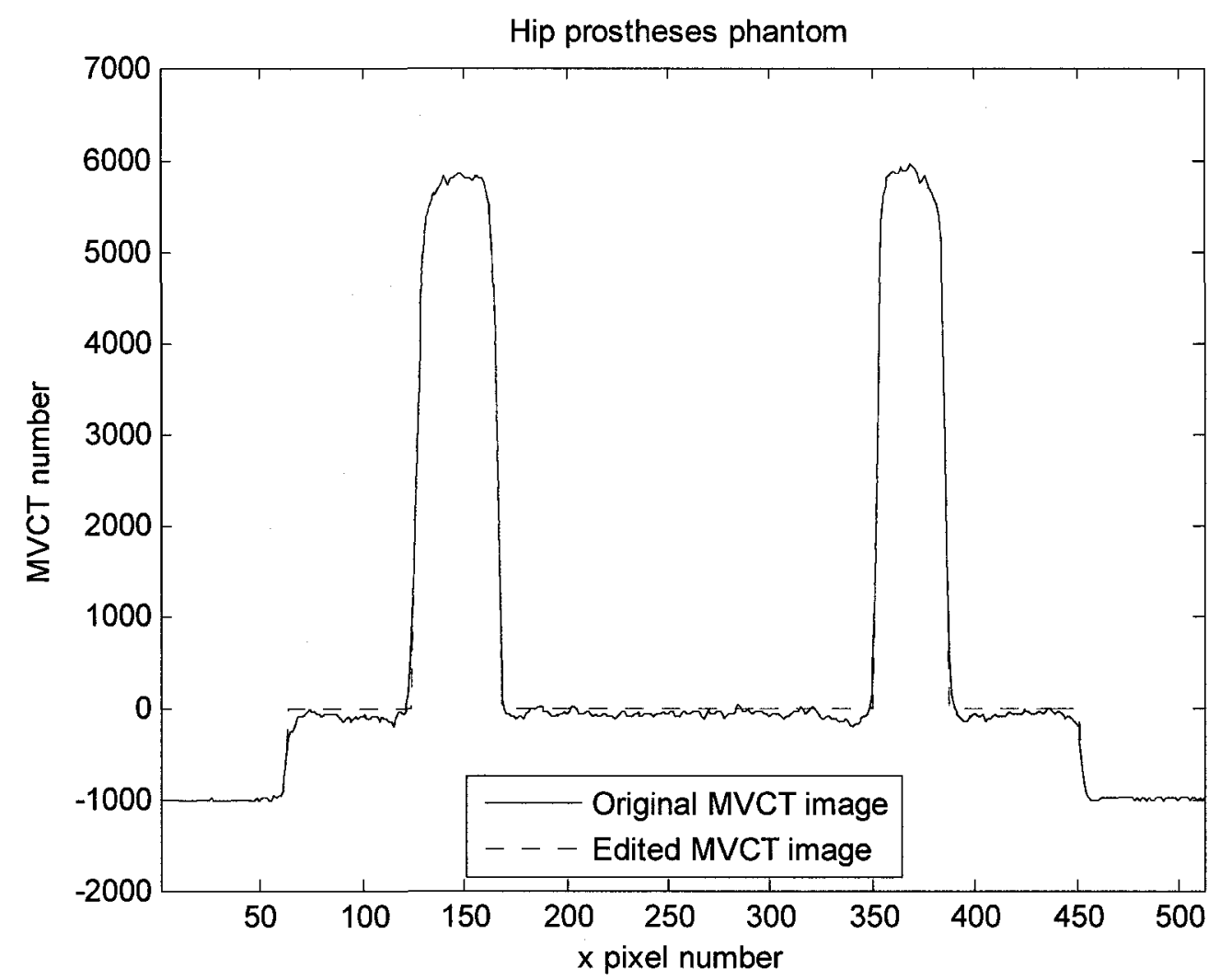

Figure 5.8 MVCT HU profiles through titanium alloy hip prostheses in a solid wax phantom before and after streak artifacts were removed from the wax region. The depth of the profile is illustrated (blue dashed line) in Figure 5.9. 
a)

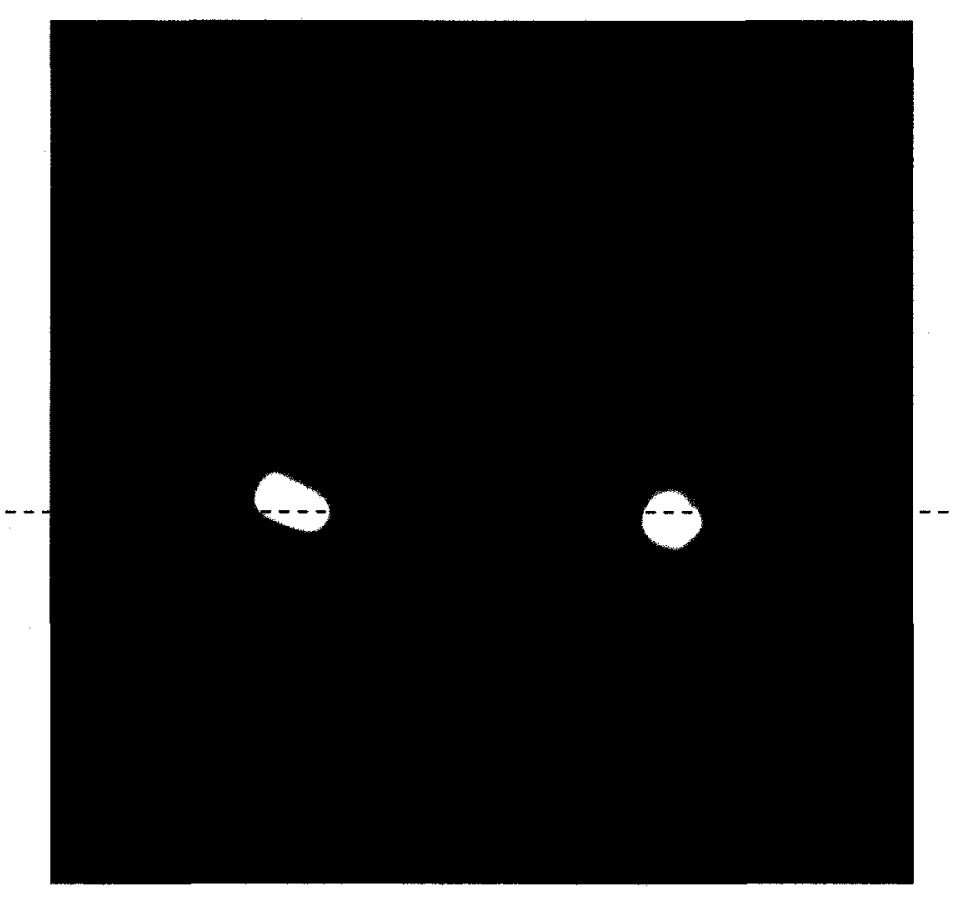

b)

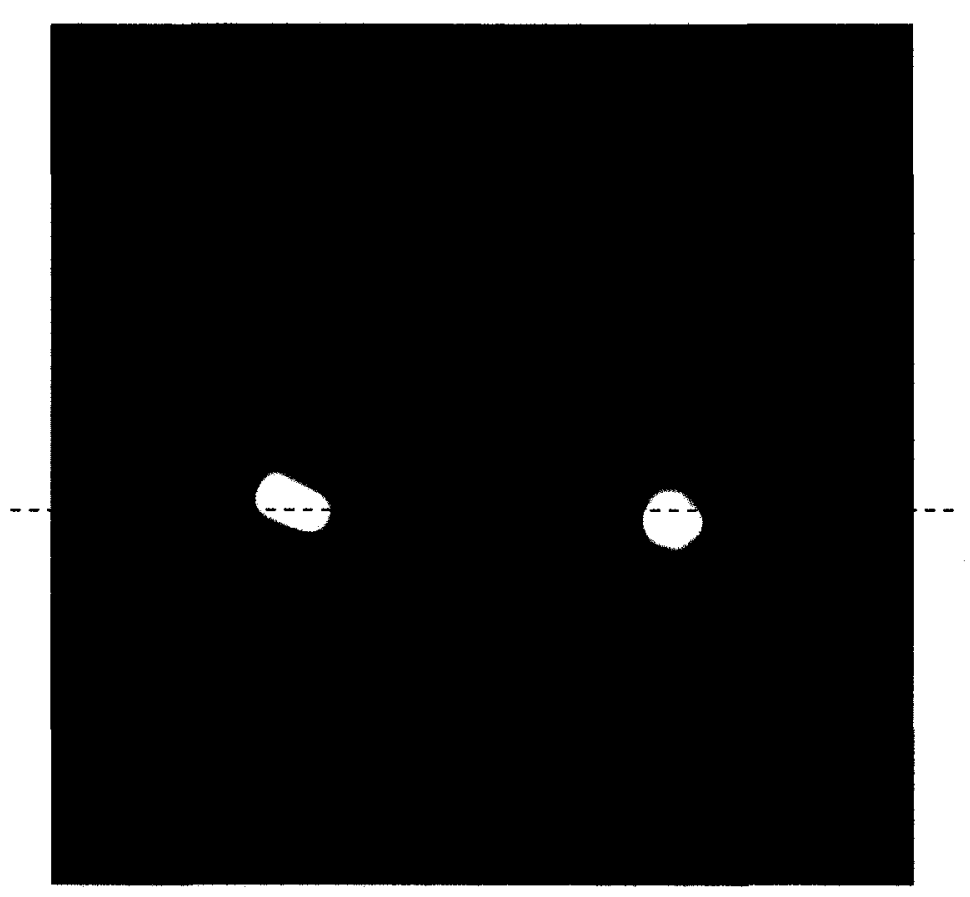

-Figure 5.9 Cross-sectional images of the hip prostheses phantom a) before and b) after streak artifacts were removed from the wax region. The blue dashed line illustrates the depth of the profiles shown in Figure 5.8 and examined in this study. 


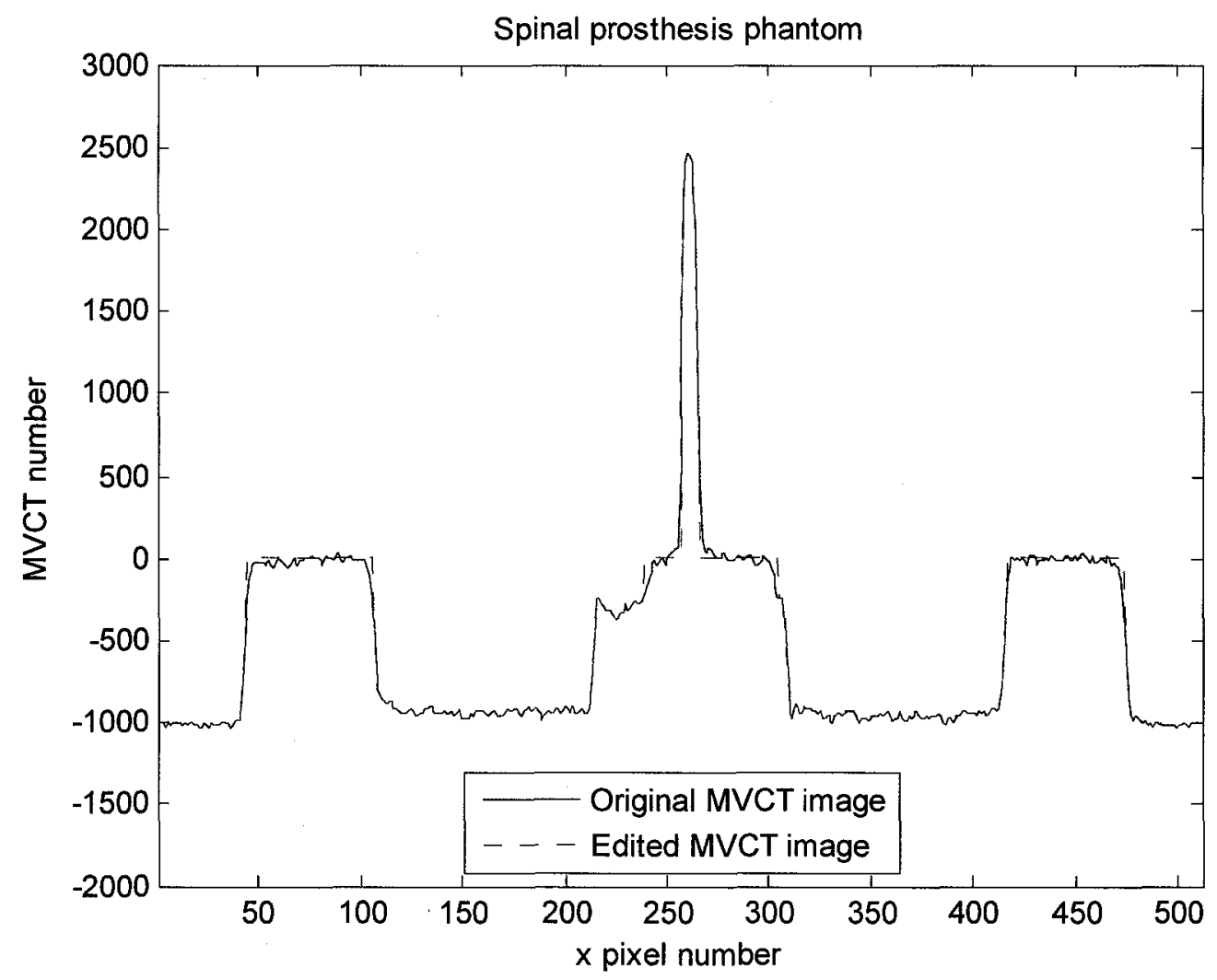

Figure 5.10 MVCT number profiles through titanium alloy spinal prosthesis and Styrofoam blocks in a solid wax phantom before and after streak artifacts were removed from the wax region. The depth of the profile is illustrated (blue dashed line) in Figure 5.11 . 
a)

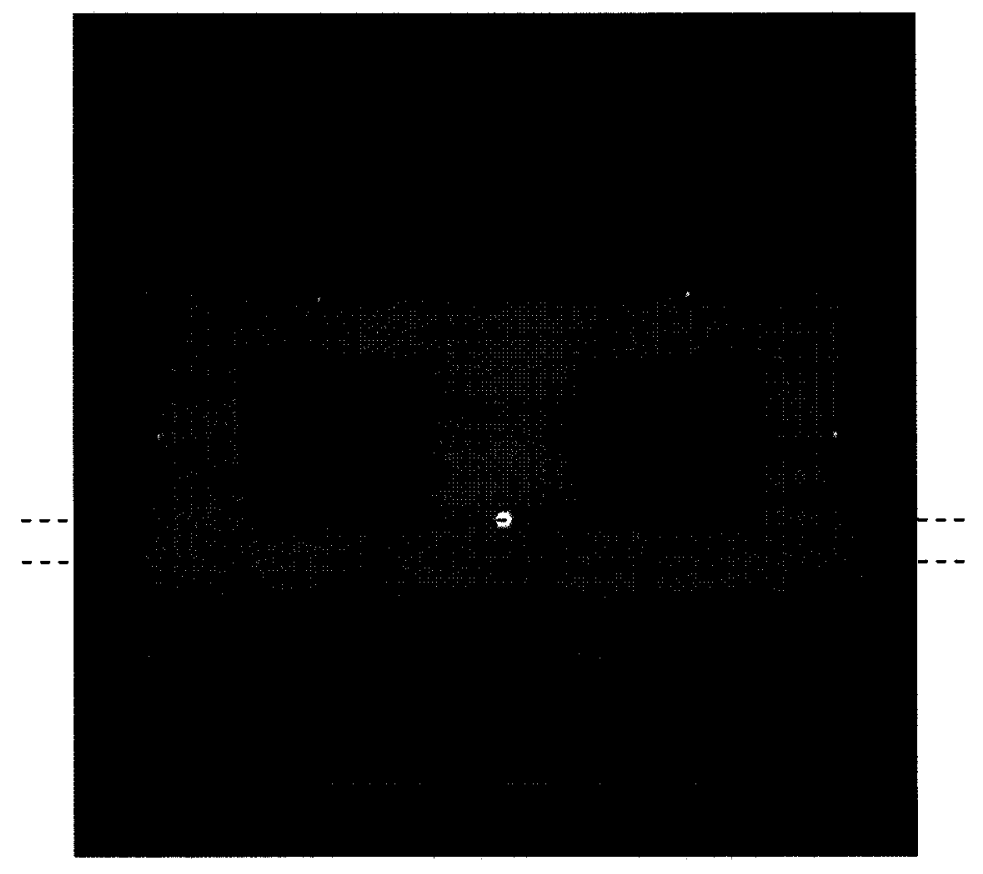

b)

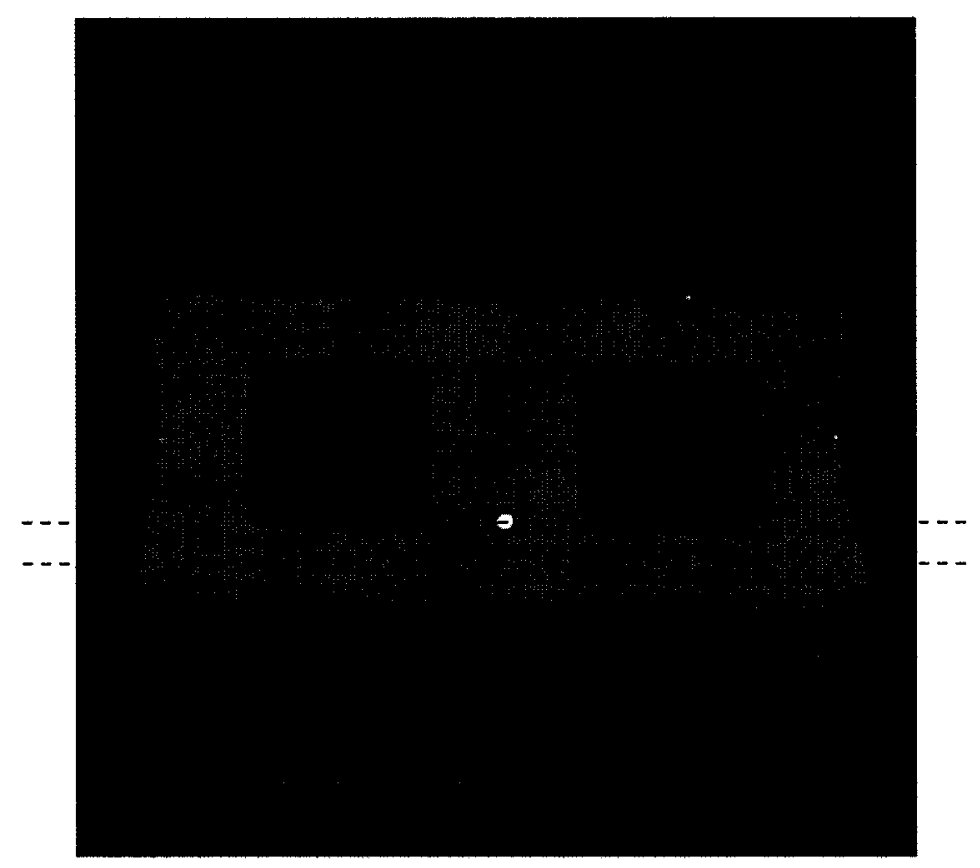

Figure 5.11 Cross-sectional images of the spinal prosthesis phantom a) before and b) after streak artifacts were removed from the wax region. The blue and red dashed lines illustrate the depths of the profiles examined in this study. 


\subsection{Results}

The pencil beam and superposition dose calculation algorithms were compared with $\mathrm{MC}$ simulations in a simple water phantom geometry to provide a baseline without CT-related dose errors. A $10 \times 10 \mathrm{~cm}^{2}$ photon beam was incident on a $30 \times 30 \times 30 \mathrm{~cm}^{3}$ water phantom at $100 \mathrm{~cm}$ SAD and $10 \mathrm{~cm}$ depth. Figures 5.12 and 5.13 show the dose profiles at $5 \mathrm{~cm}$ depth for $18-\mathrm{MV}$ and 6-MV photon energies, respectively, delivering 100 monitor units.

In this section, the dose profiles are discussed with reference to four regions within the profile. The first region is the flat area near the central axis of the geometric beam, referred to as the plateau region. The plateau region transitions into the shoulder of the profile. The dose begins to drop off within the shoulder region and it is defined here as the area between the plateau region and $80 \%$ of maximum dose. The third region is the high-dose gradient region between $20 \%$ and $80 \%$ of the central axis dose and is referred to here as the penumbra region. Finally, the last region is the fringe, defined as the area with less than $20 \%$ of central axis dose.

For visual clarity, error bars are not shown on the MC simulated dose data. The uncertainty on all MC simulated data is shown in the 6 and $18 \mathrm{MV}$ central axis depthdose curves and dose profiles shown in figures 2.4 to 2.7 in chapter 2. In high dose gradient regions, there may exist large absolute uncertainties in our calculations. These uncertainties are usually linked to geometric errors such as field size accuracy and phantom positioning. However, for our experiments, the geometry was fairly the same 
for all tests. Thus, the change from kVCT to MVCT does not necessarily have large uncertainties.

The first set of profiles examined in the hip prosthesis were for $5 \times 5 \mathrm{~cm}^{2}$ and 10 $\times 10 \mathrm{~cm}^{2} 6 \mathrm{MV}$ photon beams incident on the central axis of the hip phantom (beam setup \#1 in Figure 5.5). These are shown in Figures 5.14 and 5.15, respectively. Similar profiles for an $18 \mathrm{MV}$ photon beam are shown in Figures 5.18 and 5.19. The depths of the profiles were chosen to be at the depth of the hip prostheses since this region showed the greatest and most prominent artifacts. In this beam geometry, neither the $5 \times 5 \mathrm{~cm}^{2}$ or $10 \times 10 \mathrm{~cm}^{2}$ photon fields intersected the hip prosthesis, but they did pass through regions where the prosthesis caused image artifacts in CT image. Relative dose difference curves between the TPS and MC calculations for the two field sizes in the same beam geometry are shown in Figures 5.16 and 5.17, and 5.20 and 5.21, respectively. The difference curves show the relative deviation of the treatment planning dose predictions with the Monte Carlo calculations and were calculated as follows:

$$
\text { relativeDoseDifference }=\left(\frac{\text { Calculated }- \text { Measured }}{\text { Measured }}\right) \times 100 \%
$$

The dose data in these curves has been normalized to the EGSnrc calculated dose at the beam isocenter for each setup.

The greatest dose differences were observed in the penumbra and shoulder regions where the largest dose gradients are present. For the $6 \mathrm{MV}$ beam, differences in excess of $+/-6 \%$ are seen for the $5 \times 5$ field and in excess of $+/-10 \%$ for the $10 \times 10$ field. 
In these regions, the dose difference curves show that the kVCT based algorithms have the greatest error in dose for both field sizes and energies. In the plateau region, the kVCT-based pencil beam calculations predicted a slightly higher dose (up to 3\% difference) than the Monte Carlo simulations for the 6-MV beam. All other methods showed relatively good agreement with the gold standard for both field sizes in this region. The KVCT and MVCT pencil beam calculations reported similar doses for the 18-MV dose profiles. The $5 \times 5 \mathrm{~cm}^{2}$ profiles showed good agreement with EGSnrc calculations in the plateau region but the dose error increased progressively near the shoulders by up to $4 \%$. The $10 \times 10 \mathrm{~cm}^{2}$ pencil beam profiles consistently overestimated the dose in the plateau region by up to 3\%. The kVCT and MVCT superposition calculation showed good dose predictions near the plateau region but the $\mathrm{kVCT}$ data revealed much greater dose differences closer to the shoulder. For example, the $10 \times 10$ $\mathrm{cm}^{2} \mathrm{kVCT}$ based superposition calculations showed greater than $11 \%$ dose difference, compared to an MVCT dose difference of approximately $4 \%$. The superposition method predicted a broadened penumbra for the kVCT-based calculations relative to the MVCTbased calculations, most clearly shown in the $10 \times 10 \mathrm{~cm}^{2}$ fields for both 6 and 18-MV photon beams in Figures 5.15 and 5.19, respectively. The MVCT-based superposition calculations showed the greatest agreement to the Monte Carlo simulations.

The differences in KVCT- and MVCT-based dose calculations in the presence of a hip prosthesis was examined for a $6 \mathrm{MV}$ photon beam with a $10 \times 10 \mathrm{~cm}^{2}$ field size. Under this beam geometry, the prosthetic device was directly in the center of the geometric beam. Figures 5.22 and 5.23 show the dose profile and depth-dose curve 
through the hip prosthesis and surrounding wax. Figure 5.24 shows the difference in dose between the treatment planning algorithms and the Monte Carlo calculations for the profile shown in Figure 5.22. Neither the pencil beam nor the superposition algorithm predicted the increase in dose adjacent to the titanium prosthesis represented by the two peaks in the Monte Carlo simulation. At these peaks, the kVCT pencil beam calculations underestimated the dose by up to $17 \%$, while in the same region the MVCT pencil calculations were only slightly better with an underestimate of around $12 \%$. Both of the kVCT-based dose calculations showed irregular profiles due to the mixture of high and low density artifacts and the manner in which each algorithm accounted for these inhomogeneities. The end result was an underestimation of dose (by up to $5 \%$ ) in the surrounding wax region and an overestimation (by up to 19\%) within the prosthesis. The MVCT-based dose calculations showed better agreement with the MC simulations for both algorithms. The superposition method best followed the trend of the MC results with the exception of a $5 \%$ overdose within the prosthesis and a lack of lateral electron transport immediately adjacent to the prosthesis.

Figures 5.25, 5.26, 5.29 and 5.30 show the dose profiles of 6 and $18 \mathrm{MV}$ photon beams incident on the spinal prosthesis phantom at depth of the titanium rod. The depth of the beam profiles for the $5 \times 5 \mathrm{~cm}^{2}$ and $10 \times 10 \mathrm{~cm}^{2}$ beams is illustrated by the blue and red dashed lines, respectively, in Figure 5.11. Though it would have been informative to examine the dose at the depth of the prosthesis for the $10 \times 10 \mathrm{~cm}^{2}$ field, the data in this region was considered volatile and unreliable. The geometric beam edges included significant portions of the foam material and the spinal prosthesis is at the same 
depth as the distal foam-wax interface where voxel averaging effects will introduce significant uncertainties. The combination of a high-dose gradient region and volume average effects resulted in erratic profiles at the depth of the prosthesis. Thus, a depth below the prosthesis was chosen to examine the impact of the high $\mathrm{Z}$ material in a more stable region. Analysis showed minimal electron density differences between the kVCT and MVCT images. The superposition predicted more accurate doses than the pencil beam data for the $10 \times 10 \mathrm{~cm}^{2}$ beams. The pencil beam data begins to overestimate the dose in the low $\mathrm{Z}$ and wax regions by up to $8 \%$ and $4 \%$, respectively, at high energies. 


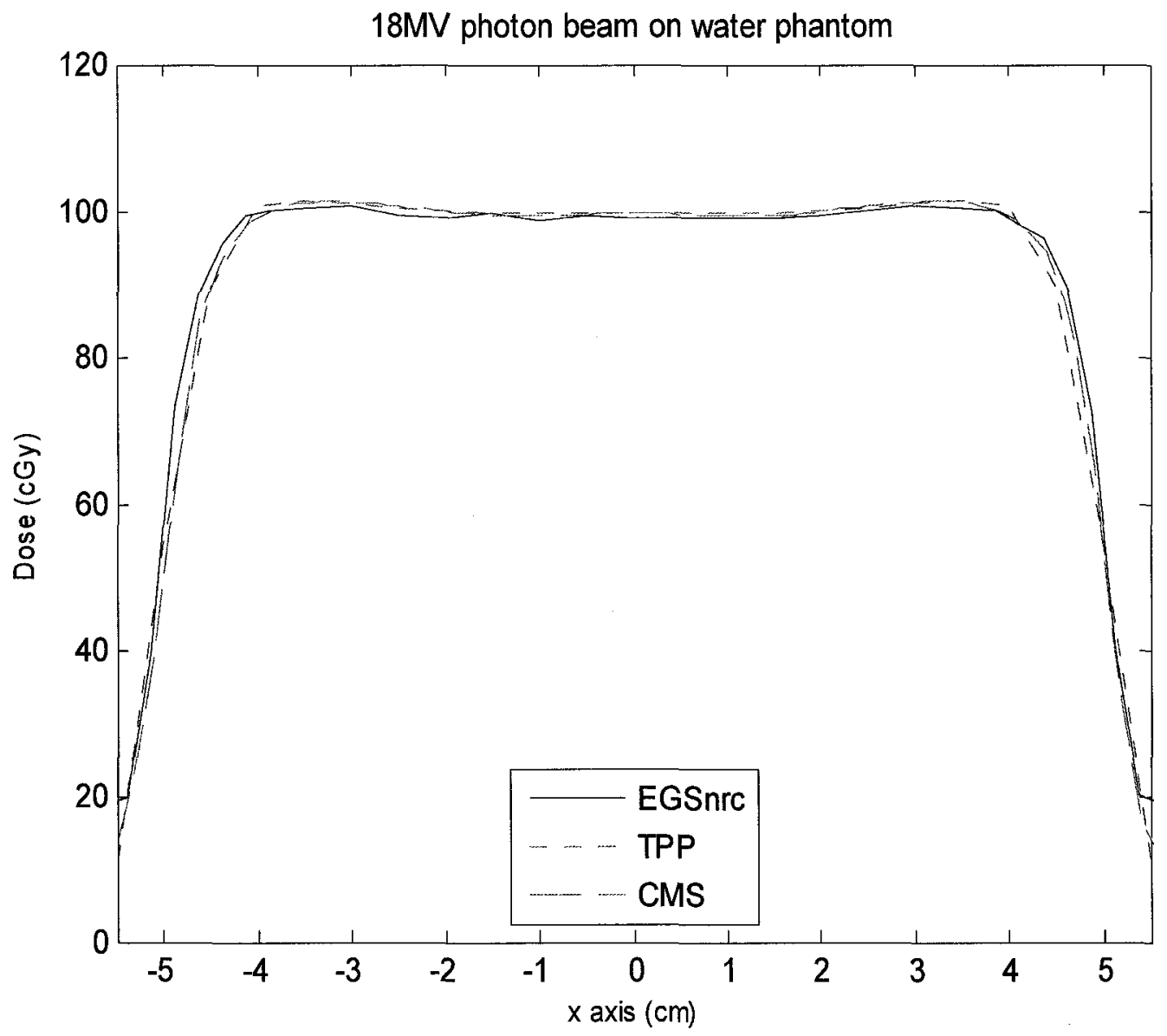

Figure 5.12 Comparing treatment planning system (Theraplan Plus v3.8 and CMS) and Monte Carlo based EGSnrc dose calculations in a $30 \times 30 \times 30 \mathrm{~cm}^{3}$ water phantom for an $18 \mathrm{MV}, 10 \times 10 \mathrm{~cm}^{2}$ field at $100 \mathrm{~cm} \mathrm{SAD}(10 \mathrm{~cm}$ depth $)$ delivering $100 \mathrm{MUs}$. 


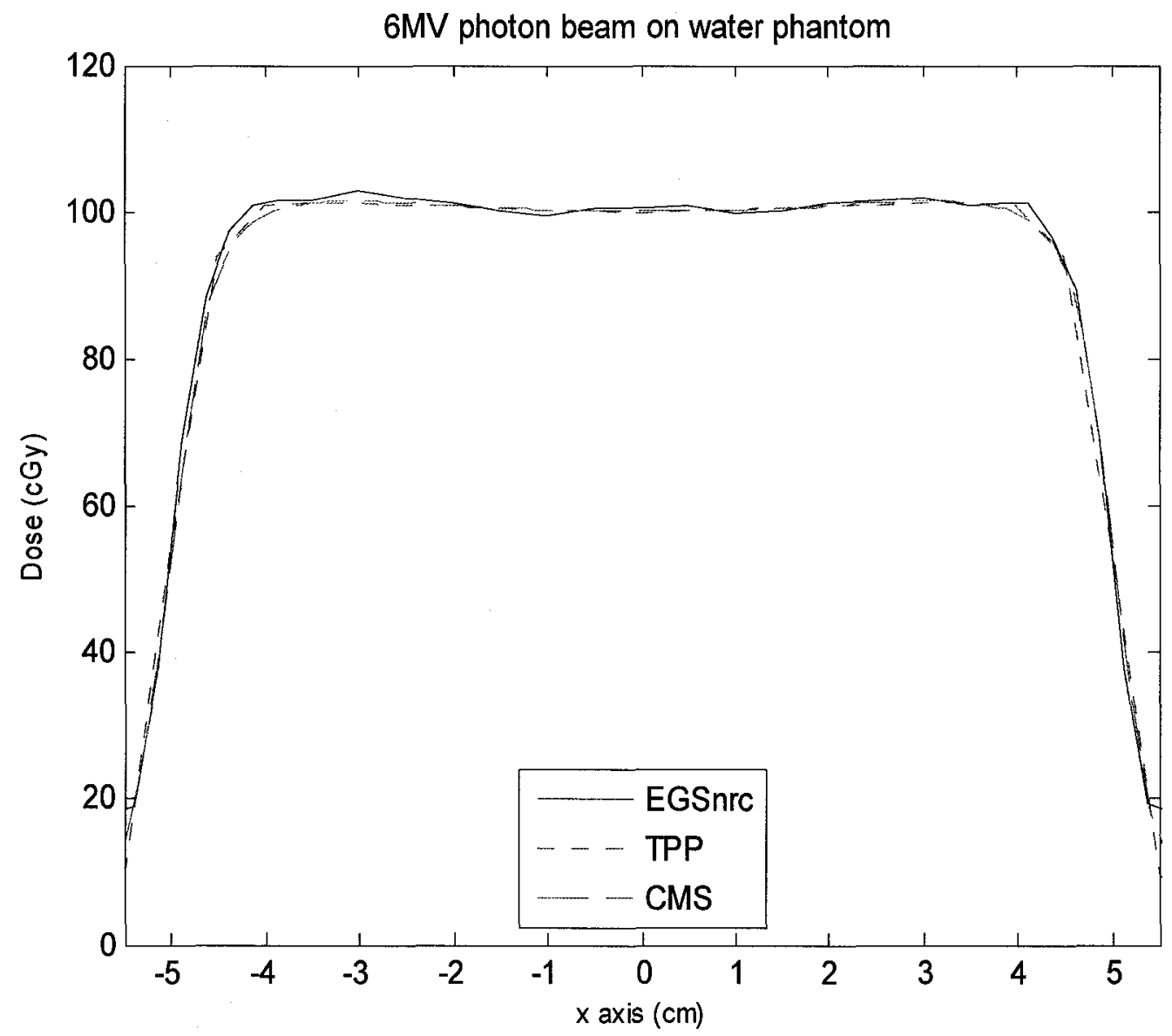

Figure 5.13 Comparing treatment planning system (Theraplan Plus v3.8 and CMS) and Monte Carlo based EGSnrc dose calculations in a $30 \times 30 \times 30 \mathrm{~cm}^{3}$ water phantom for an $18 \mathrm{MV}, 10 \times 10 \mathrm{~cm}^{2}$ field at $100 \mathrm{~cm} \mathrm{SAD}(10 \mathrm{~cm}$ depth) delivering $100 \mathrm{MUs}$. 


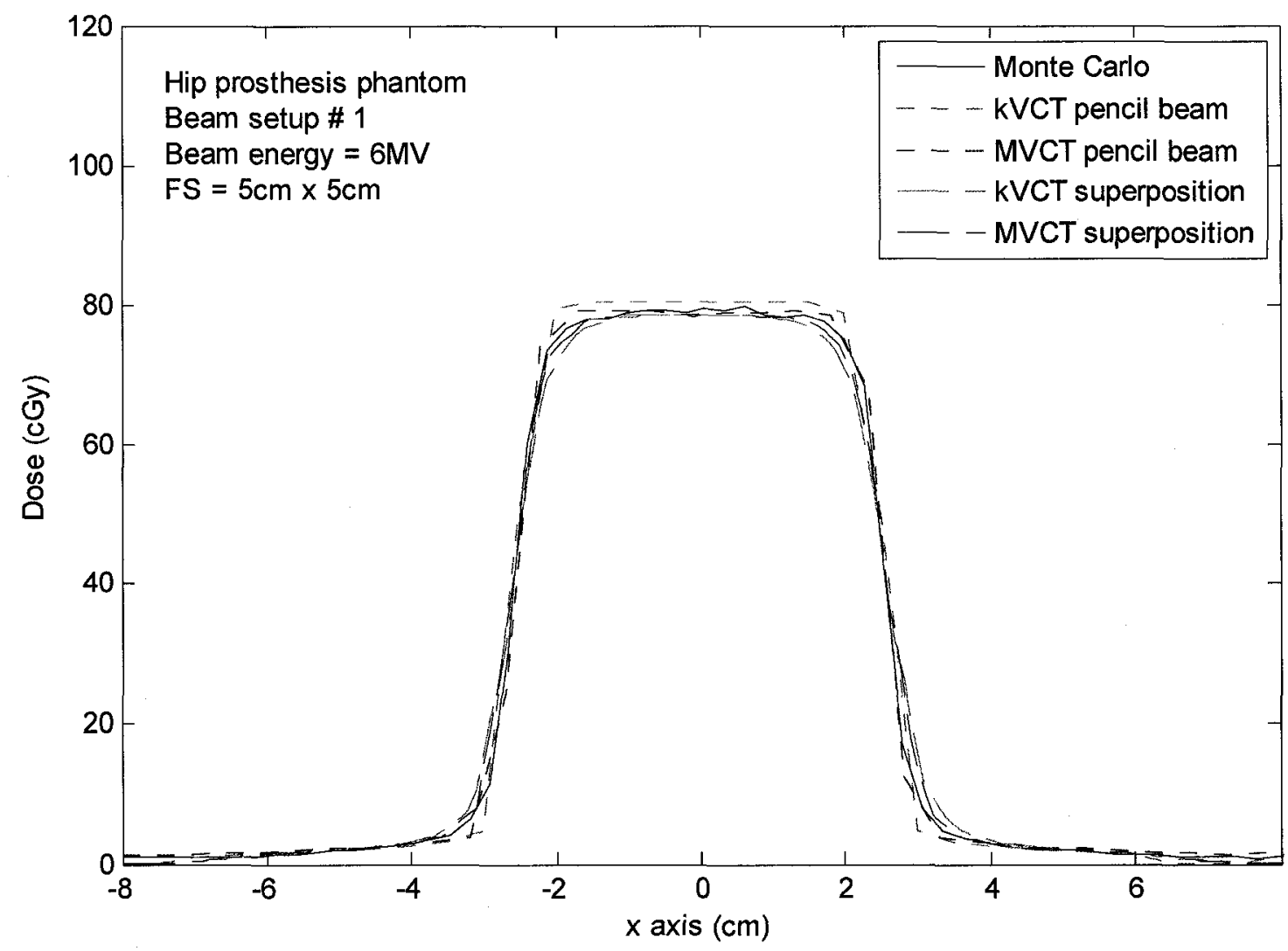

Figure 5.14 kVCT and MVCT based dose profiles calculated with Monte Carlo, pencil beam and superposition algorithms for a $5 \times 5 \mathrm{~cm}^{2} 6 \mathrm{MV}$ photon beam incident on the central axis (beam setup \#1 in Figure 5.5) of a hip prostheses phantom. The depth of the profiles was chosen to intersect the prosthesis as shown in Figure 5.8. 


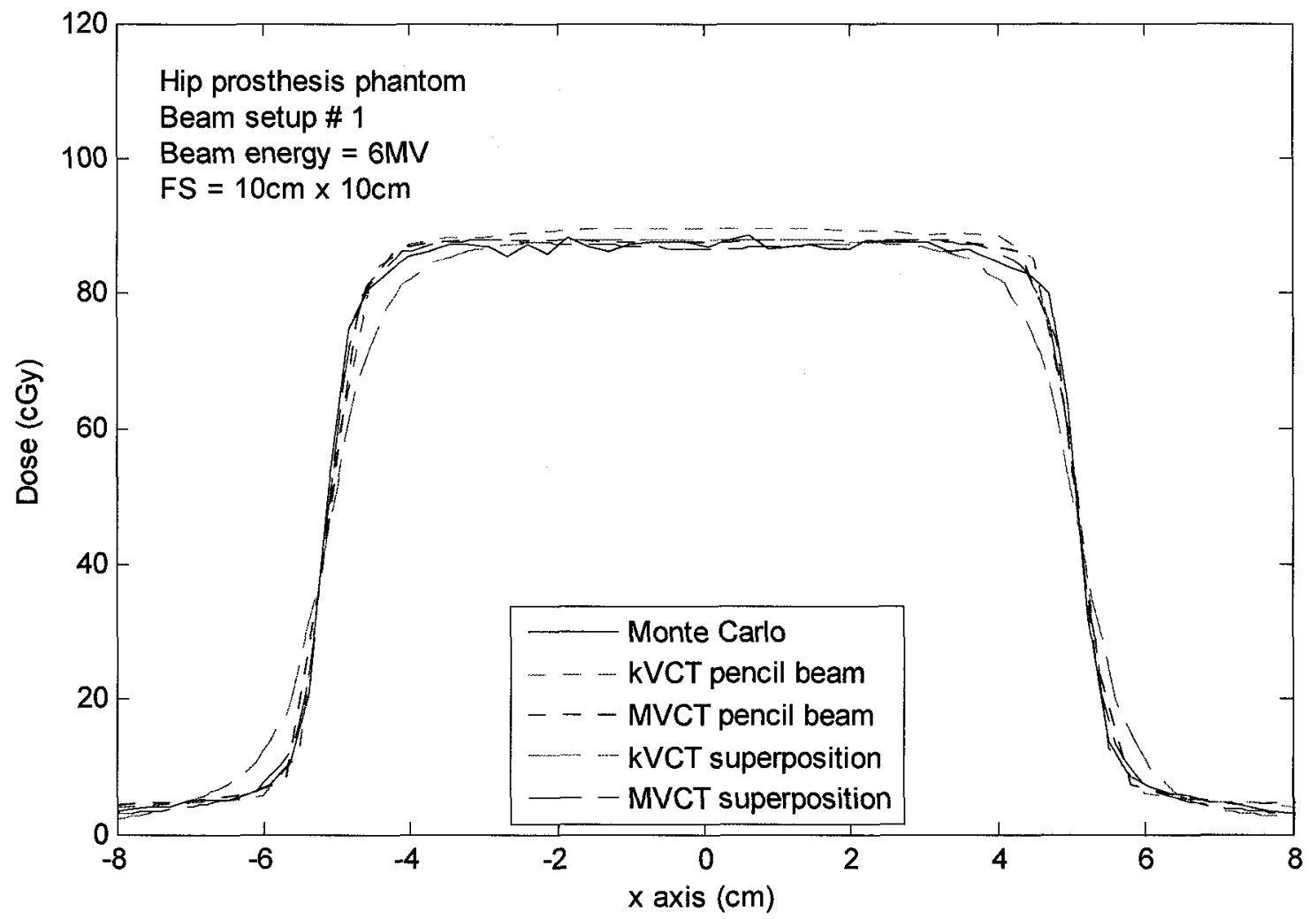

Figure 5.15 kVCT and MVCT based dose profiles calculated with Monte Carlo, pencil beam and superposition algorithms for a $10 \times 10 \mathrm{~cm}^{2} 6 \mathrm{MV}$ photon beam incident on the central axis (beam setup \#1 in Figure 5.5) of a hip prostheses phantom. The depth of the profiles was chosen to intersect the prosthesis as shown in Figure 5.8. 


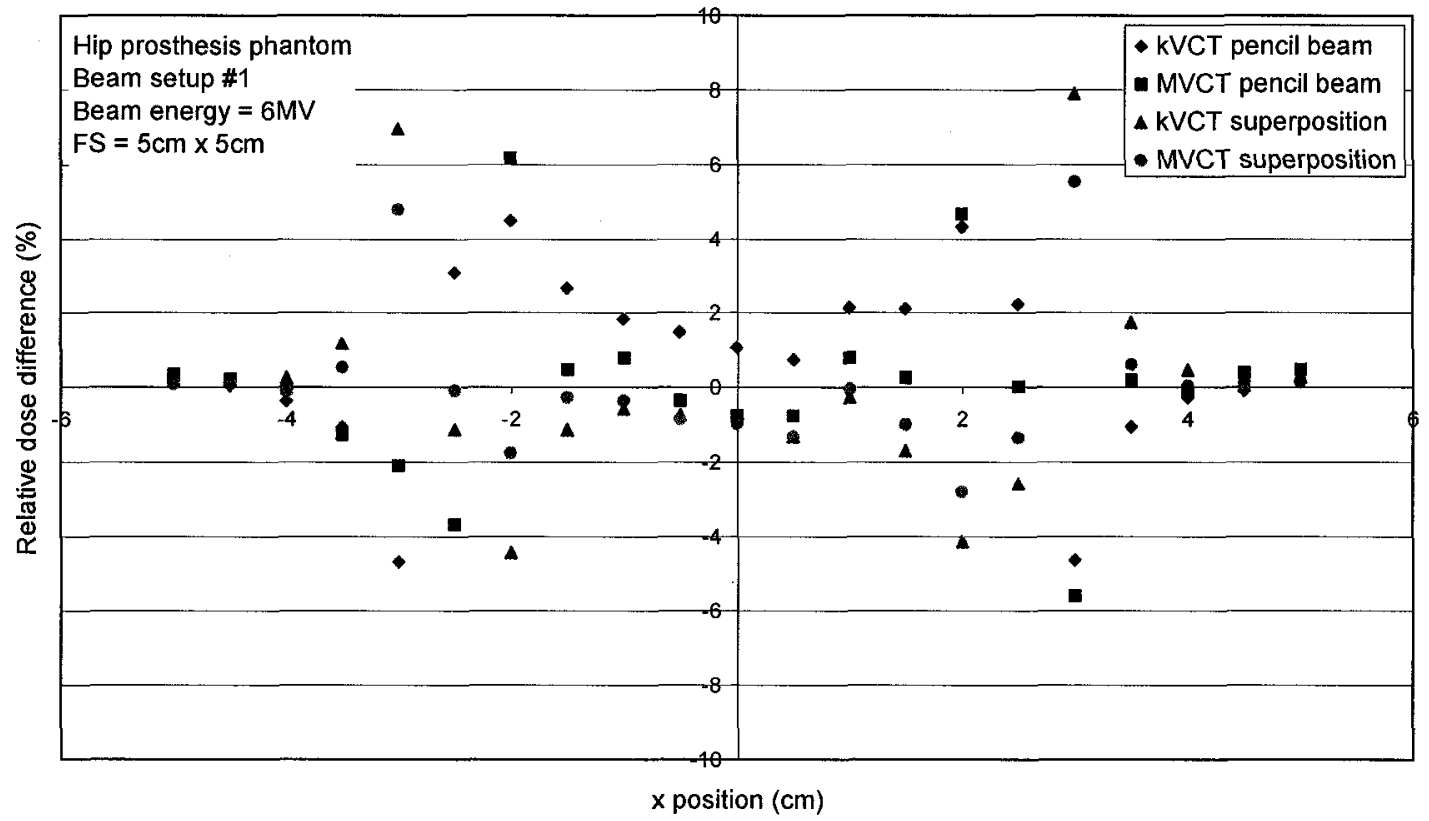

Figure 5.16 The dose difference between treatment planning calculations (pencil beam and superposition algorithms) and MC simulations for a $5 \times 5 \mathrm{~cm}^{2} 6 \mathrm{MV}$ photon beam incident on the central axis (beam setup \#1 in Figure 5.5) of a hip prostheses phantom. The depth of the profiles is shown in Figure 5.8. 


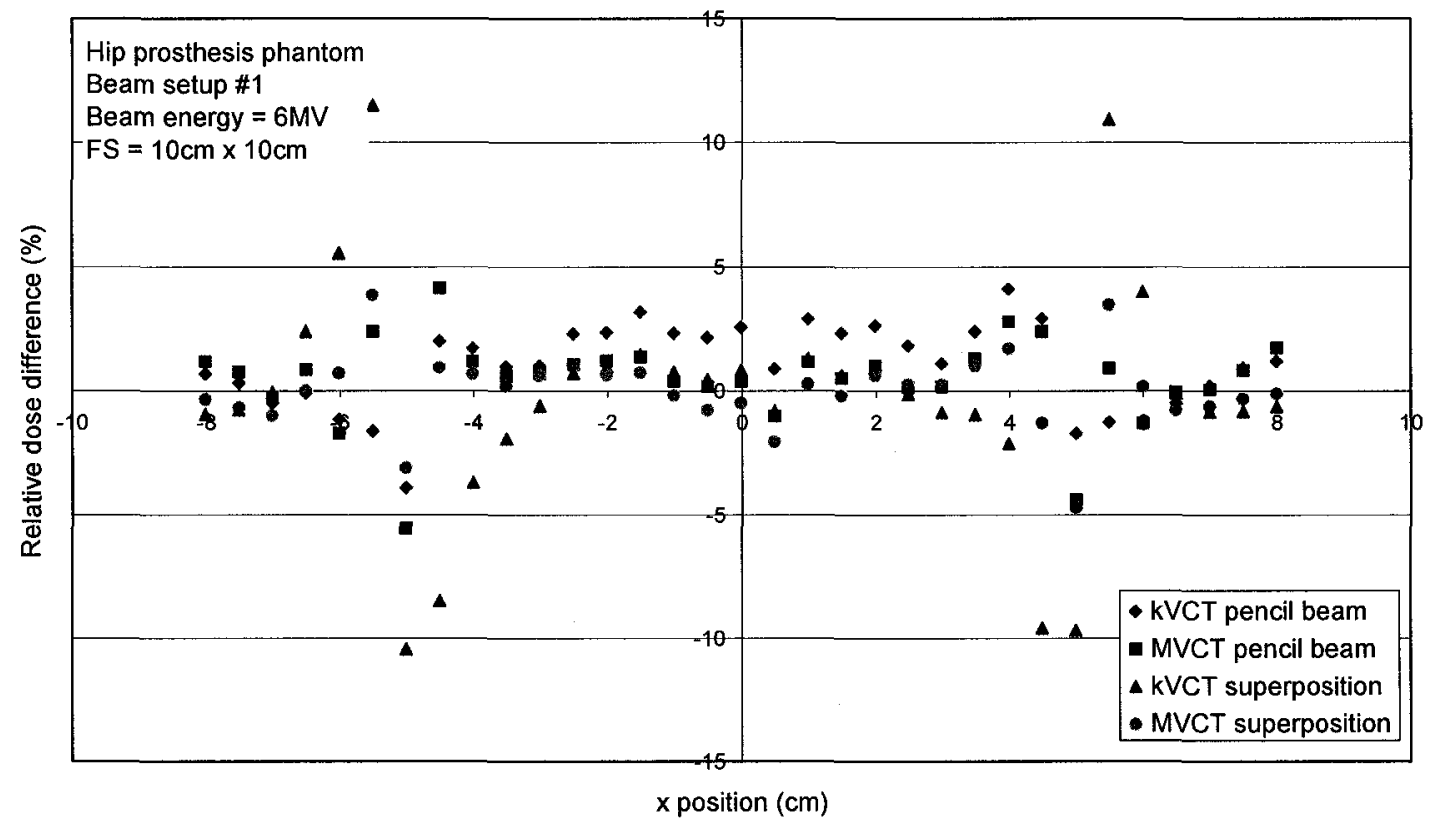

Figure 5.17 The dose difference between treatment planning calculations (pencil beam and superposition algorithms) and MC simulations for a $10 \times 10 \mathrm{~cm}^{2} 6 \mathrm{MV}$ photon beam incident on the central axis (beam setup \#1 in Figure 5.5) of a hip prostheses phantom. The depth of the profiles is shown in Figure 5.8. 


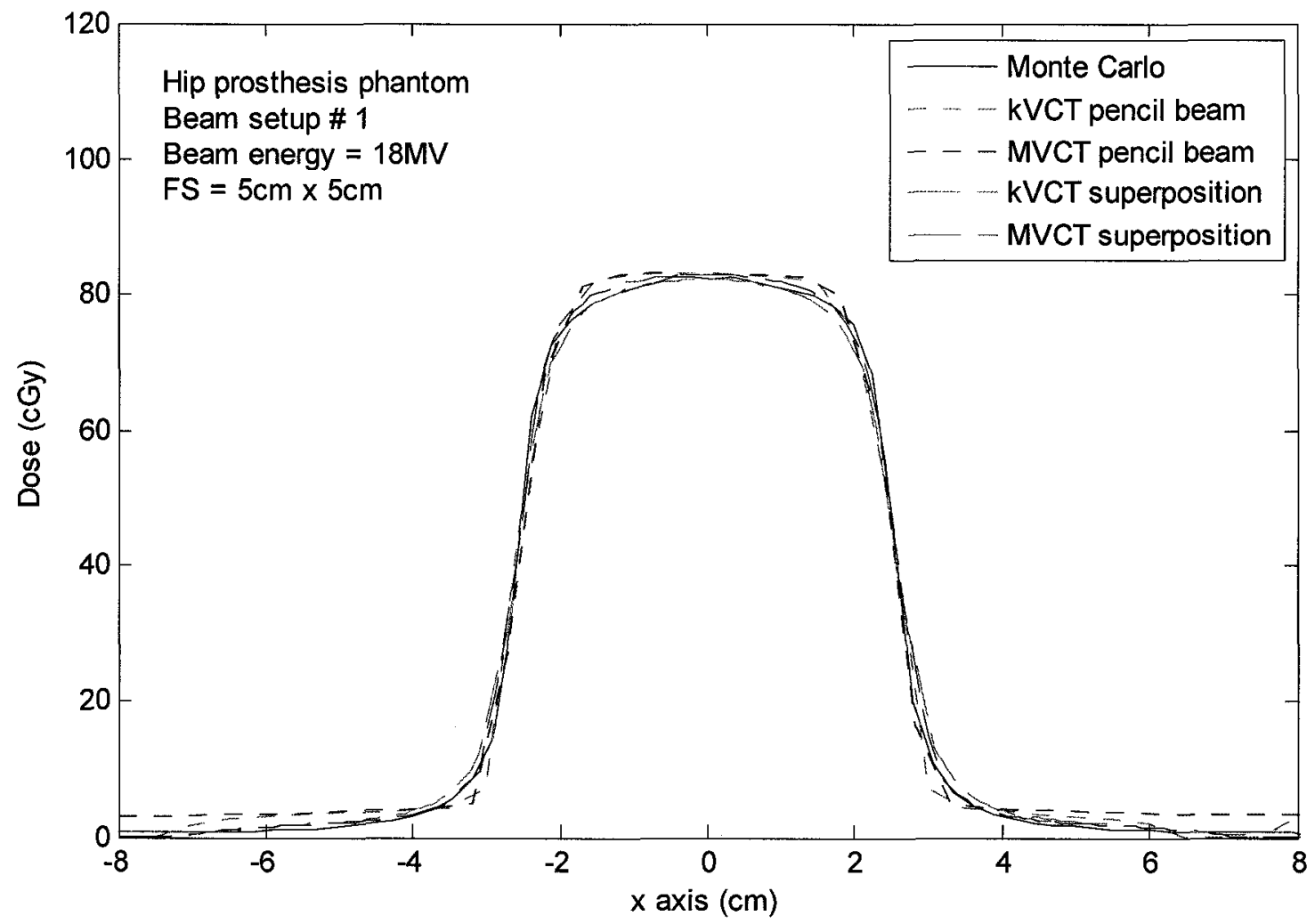

Figure 5.18 kVCT and MVCT based dose profiles calculated with Monte Carlo, pencil beam and superposition algorithms for a $5 \times 5 \mathrm{~cm}^{2} 18 \mathrm{MV}$ photon beam incident on the central axis (beam setup \#1 in Figure 5.5) of a hip prostheses phantom. The depth of the profiles was chosen to intersect the prosthesis as shown in Figure 5.8. 


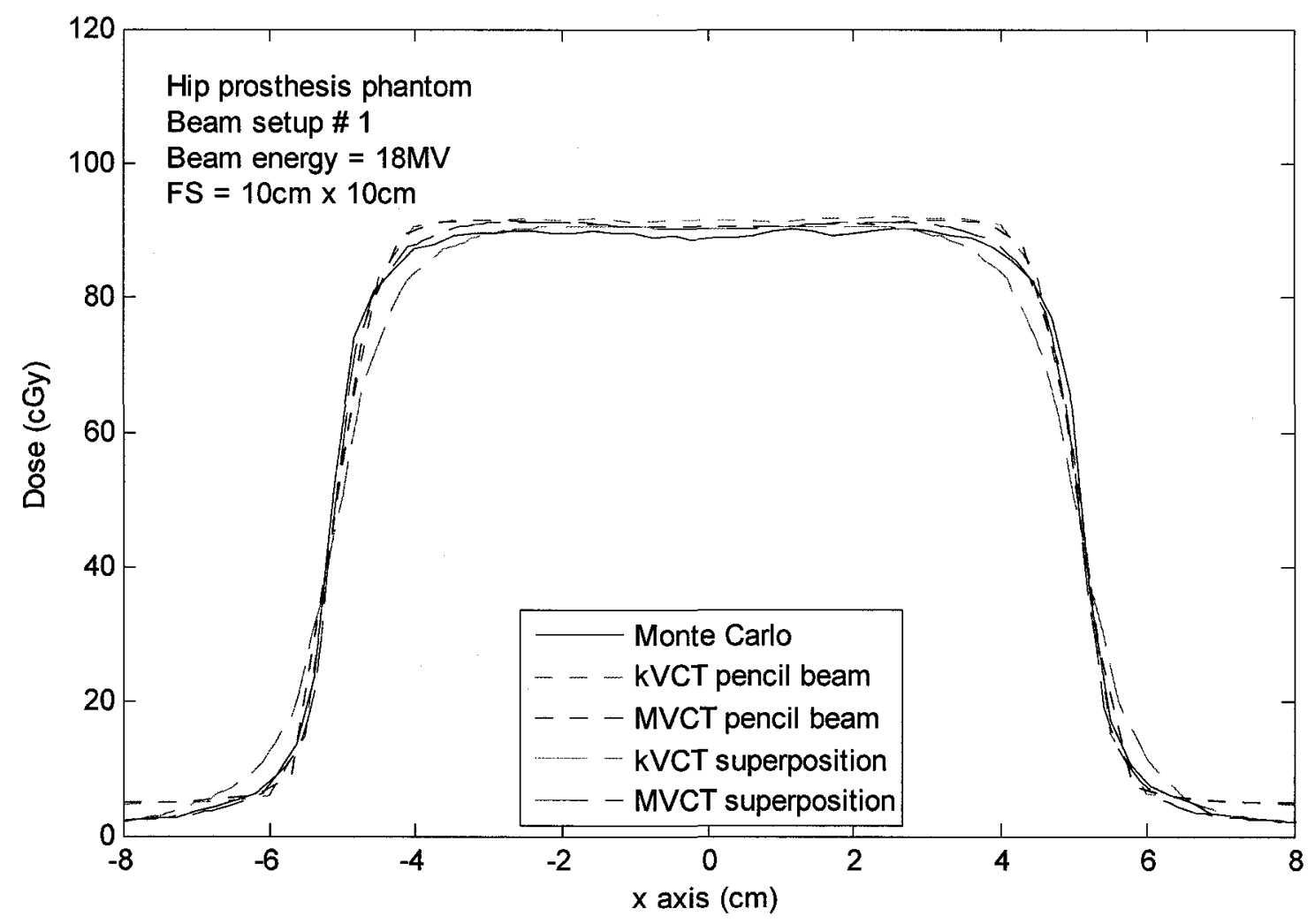

Figure 5.19 kVCT and MVCT based dose profiles calculated with Monte Carlo, pencil beam and superposition algorithms for a $10 \times 10 \mathrm{~cm}^{2} 18 \mathrm{MV}$ photon beam incident on the central axis (beam setup \#1 in Figure 5.5) of a hip prostheses phantom. The depth of the profiles was chosen to intersect the prosthesis as shown in Figure 5.8. 


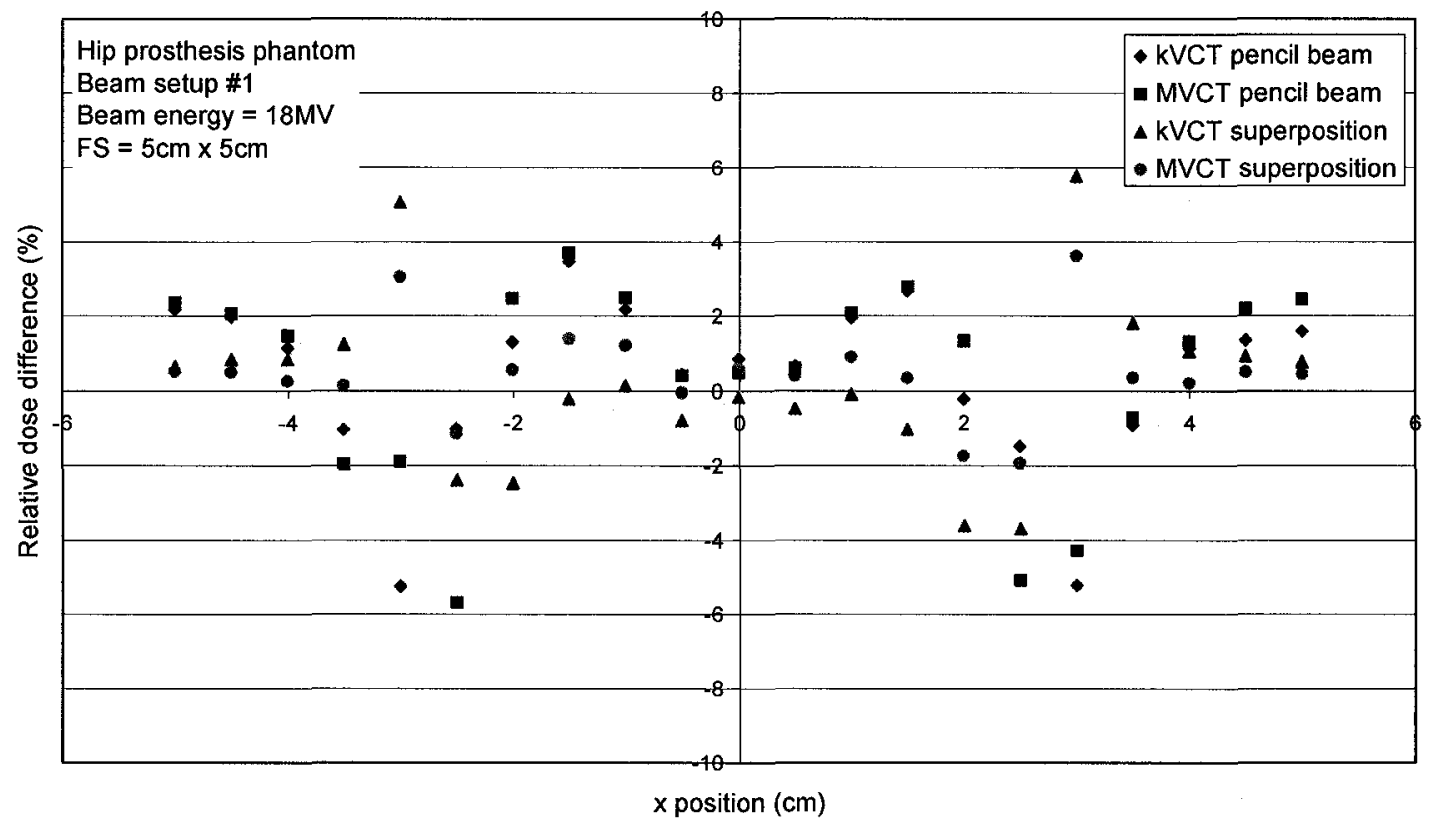

Figure 5.20 The dose difference between treatment planning calculations (pencil beam and superposition algorithms) and MC simulations for a $5 \times 5 \mathrm{~cm}^{2} 18 \mathrm{MV}$ photon beam incident on the central axis (beam setup \#1 in Figure 5.5) of a hip prostheses phantom. The depth of the profiles is shown in Figure 5.8. 


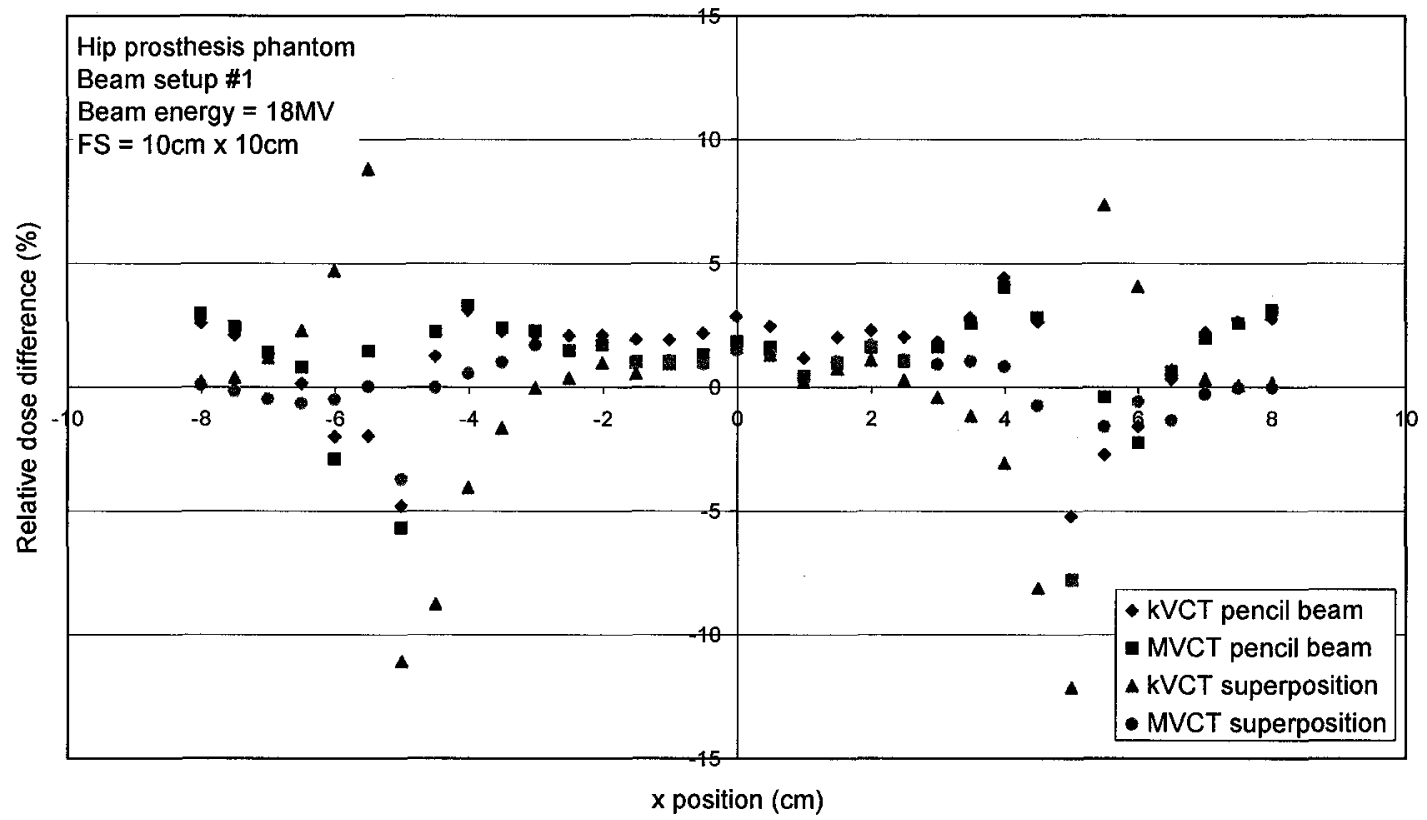

Figure 5.21 The dose difference between treatment planning calculations (pencil beam and superposition algorithms) and MC simulations for a $10 \times 10 \mathrm{~cm}^{2} 18 \mathrm{MV}$ photon beam incident on the central axis (beam setup \#1 in Figure 5.5) of a hip prostheses phantom. The depth of the profiles is shown in Figure 5.8. 


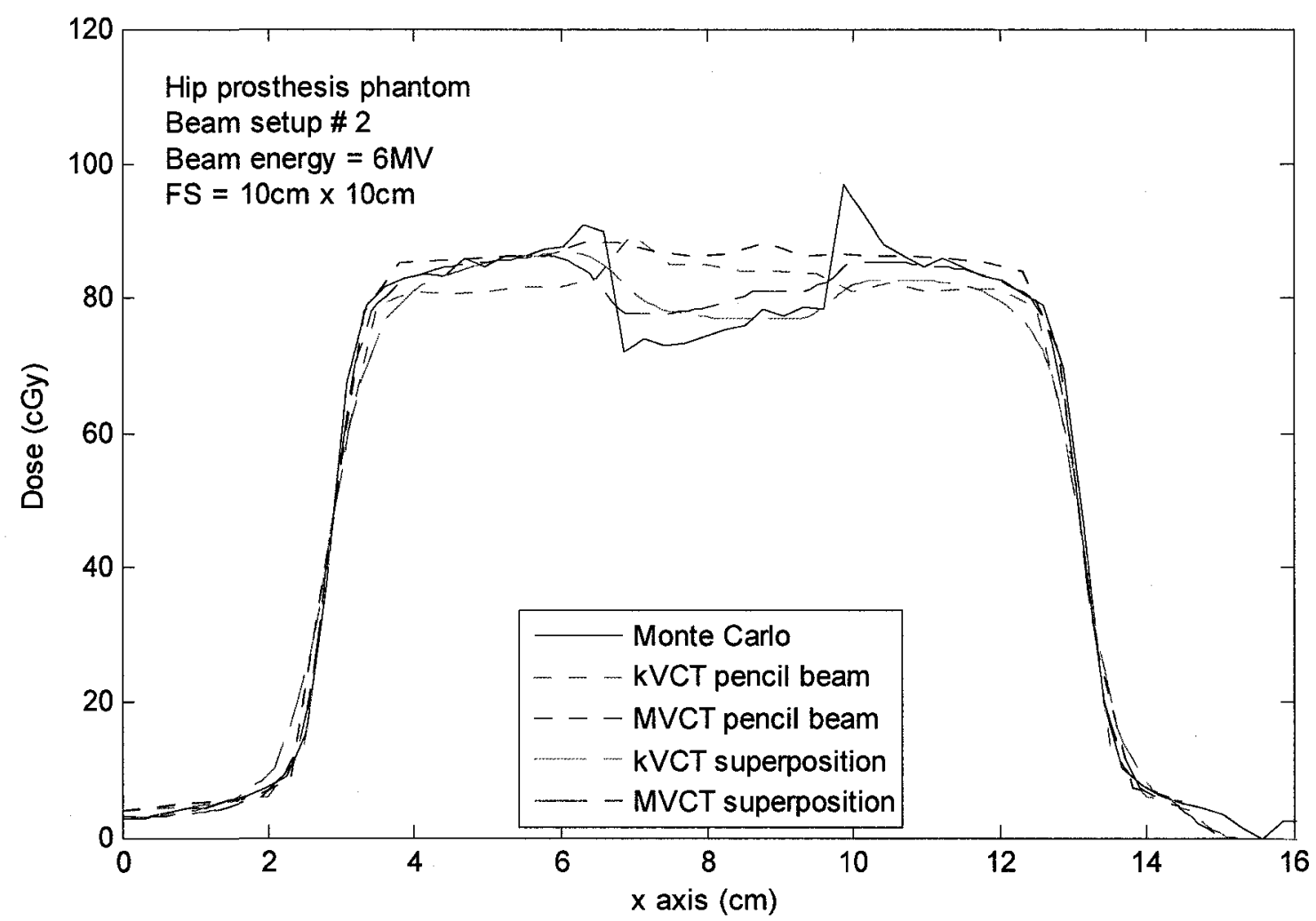

Figure 5.22 kVCT and MVCT based dose profiles calculated with Monte Carlo, pencil beam and superposition algorithms for a $10 \times 10 \mathrm{~cm}^{2} 6 \mathrm{MV}$ photon beam incident on the hip prosthesis at the central axis depth (beam setup \#2 in Figure 5.5) of a wax phantom. The depth of the profiles is shown in Figure 5.8 


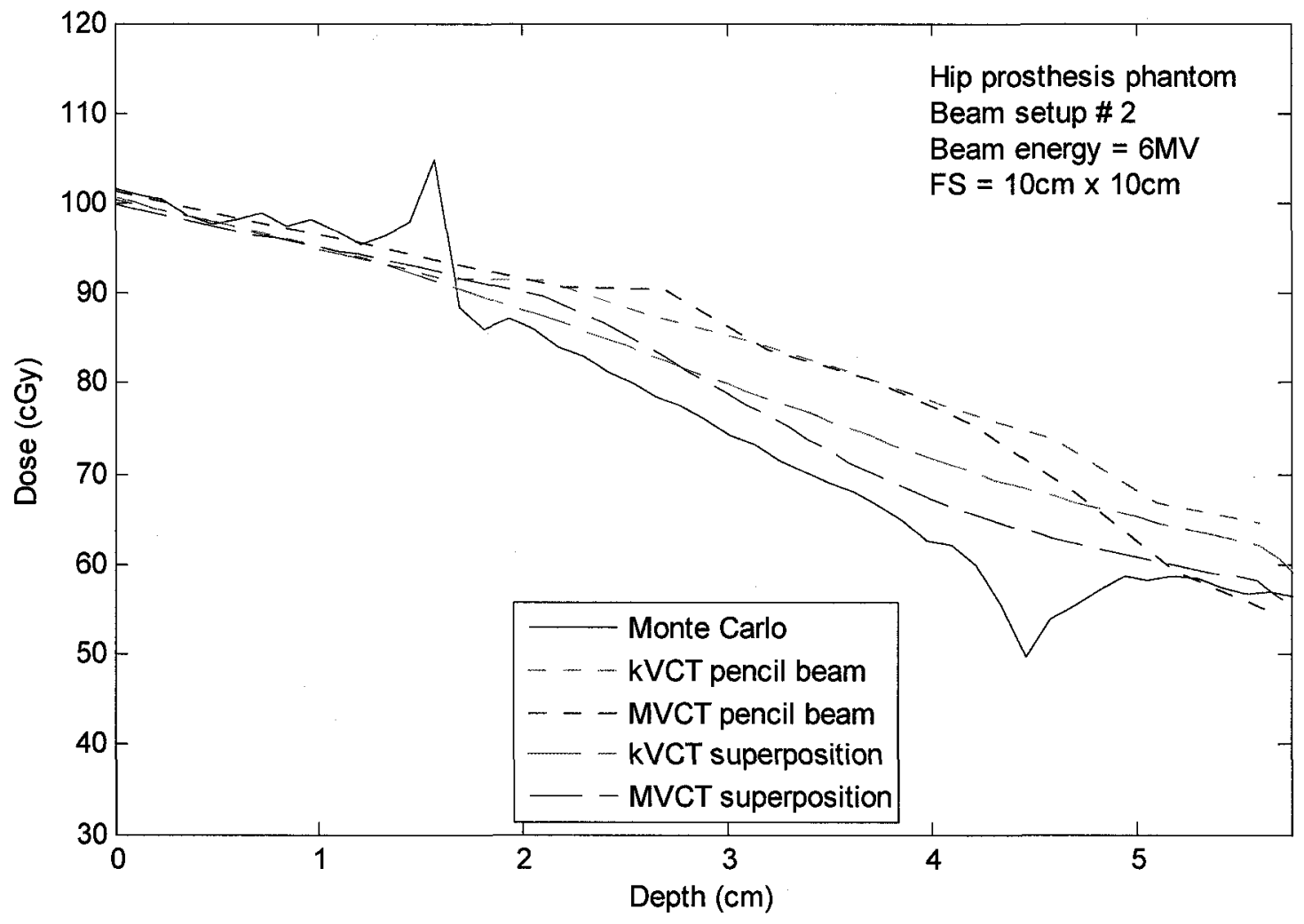

Figure 5.23 kVCT and MVCT based depth-dose profiles calculated with Monte Carlo, pencil beam and superposition algorithms for a $10 \times 10 \mathrm{~cm}^{2} 6 \mathrm{MV}$ photon beam incident on the hip prosthesis at the central axis depth (beam setup $\# 2$ in Figure 5.5) of a wax phantom. The depth of the profiles is shown in Figure 5.8. 


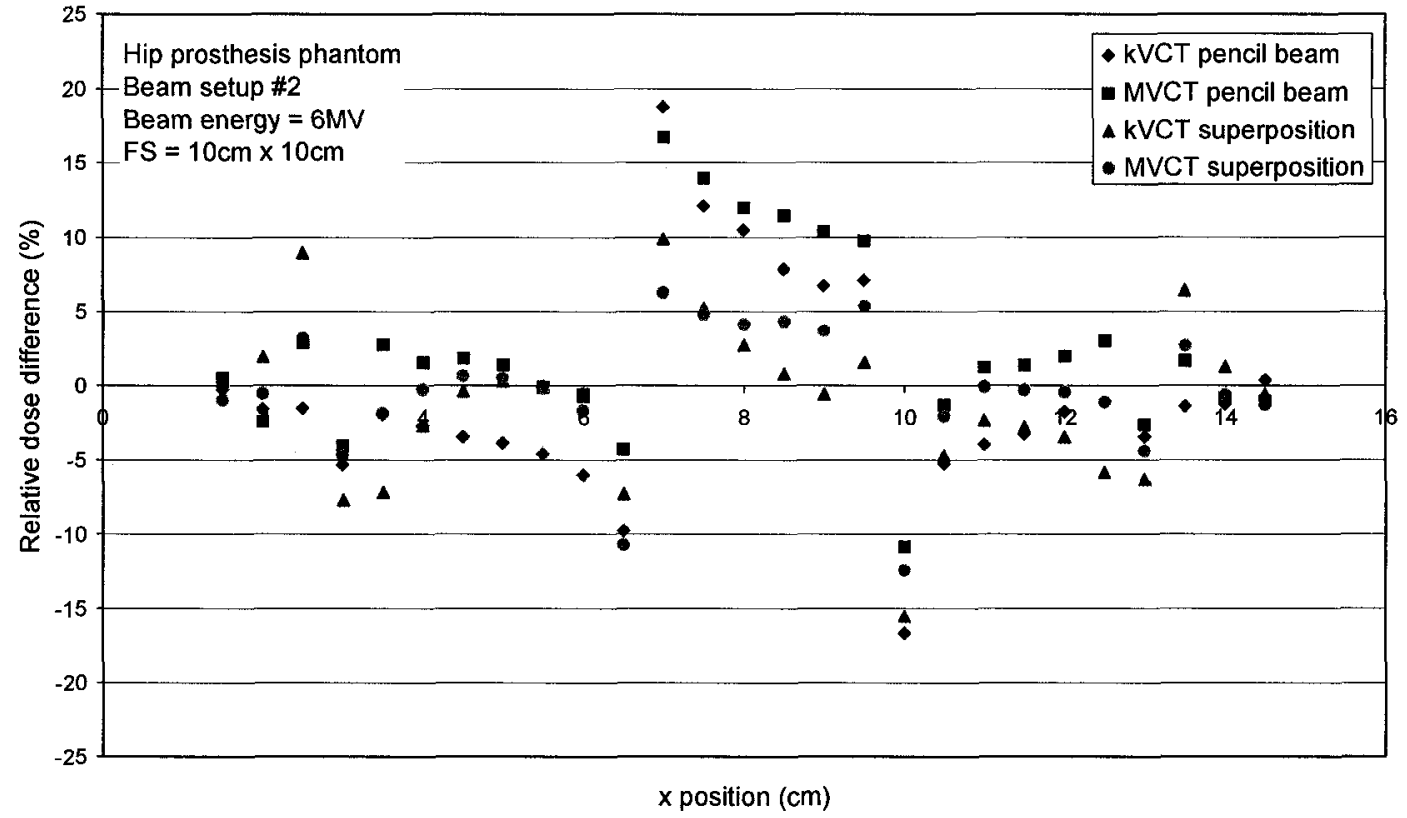

Figure 5.24 The dose difference between treatment planning calculations (pencil beam and superposition algorithms) and $\mathrm{MC}$ simulations for a $10 \times 10 \mathrm{~cm}^{2} 6 \mathrm{MV}$ photon beam incident on the hip prosthesis at the central axis depth (beam setup \#2 in Figure 5.5) of a wax phantom. The depth of the profiles is shown in Figure 5.8. 


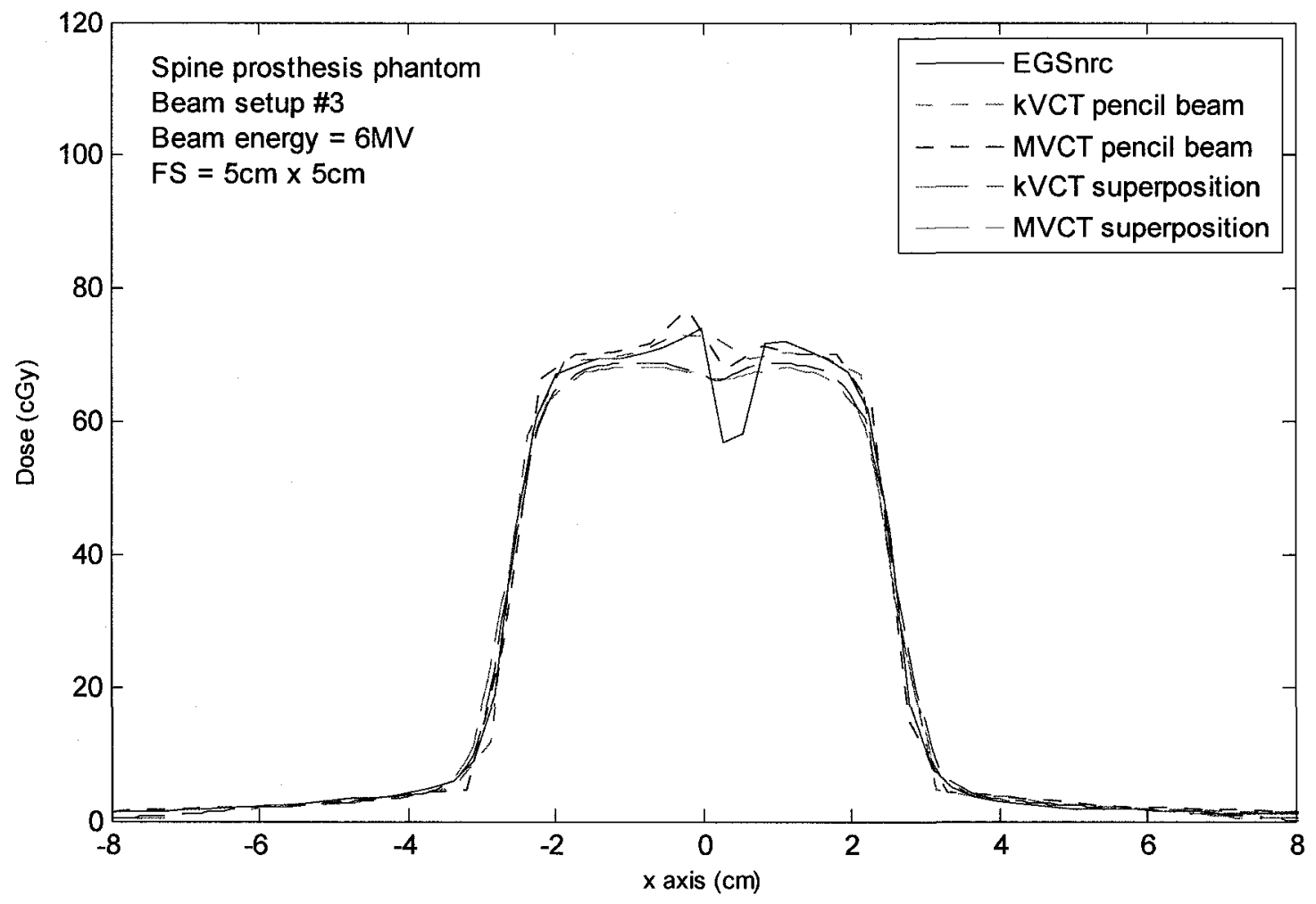

Figure 5.25 kVCT and MVCT based dose profiles calculated with Monte Carlo, pencil beam and superposition algorithms for a $5 \times 5 \mathrm{~cm}^{2} 6 \mathrm{MV}$ photon beam incident on the central axis (beam setup \#3 in Figure 5.5) of a spinal prosthesis phantom. The depth of the profiles was chosen to intersect the prosthesis as illustrated by the blue dashed line in Figure 5.10. 


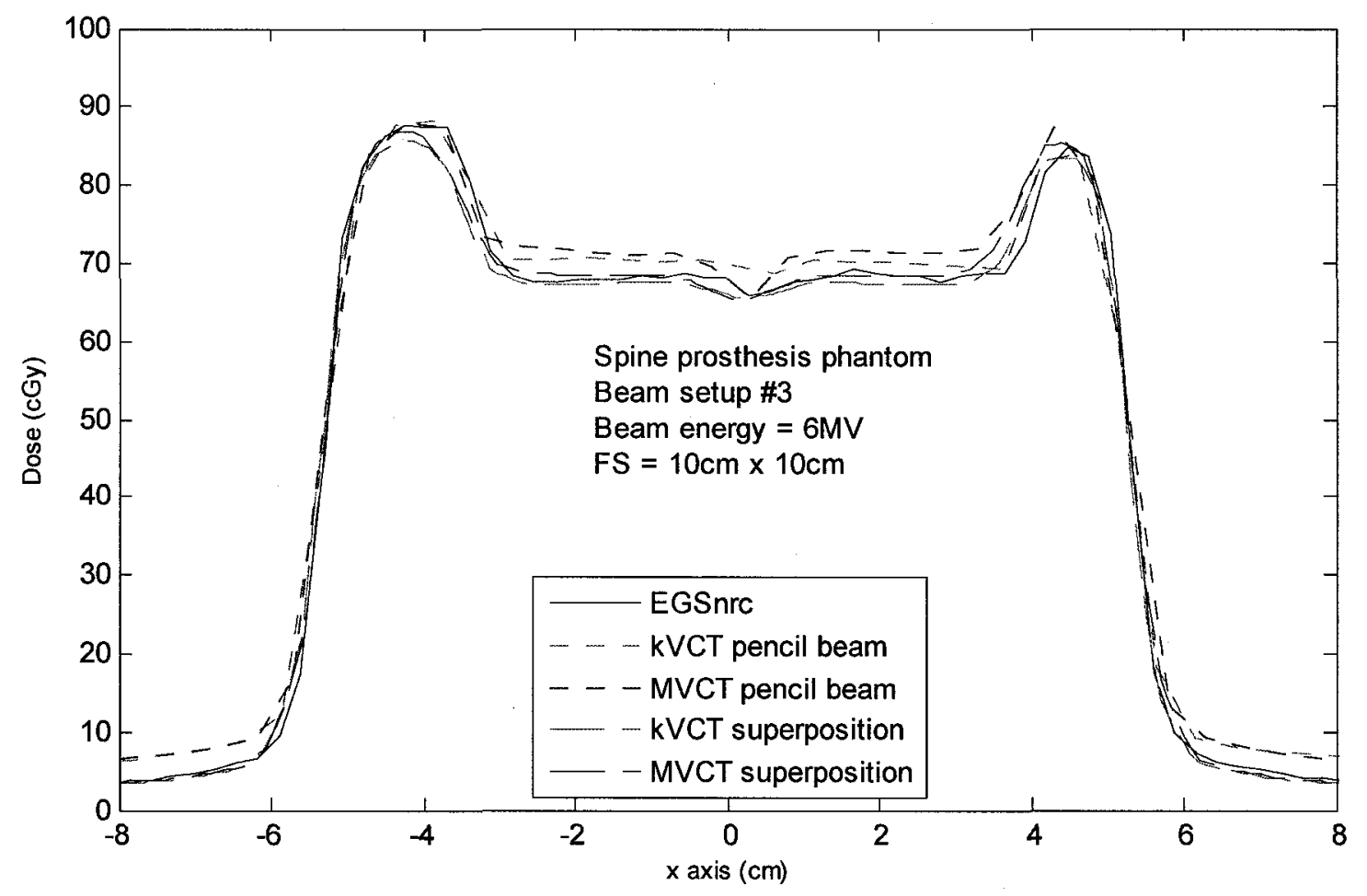

Figure 5.26 kVCT and MVCT based dose profiles calculated with Monte Carlo, pencil beam and superposition algorithms for a $10 \times 10 \mathrm{~cm}^{2} 6 \mathrm{MV}$ photon beam incident on the central axis (beam setup \#3 in Figure 5.5) of a spinal prosthesis phantom. The depth of the profiles was $2 \mathrm{~cm}$ distal to the spinal rod as illustrated by the red dashed line in Figure 5.10 . 


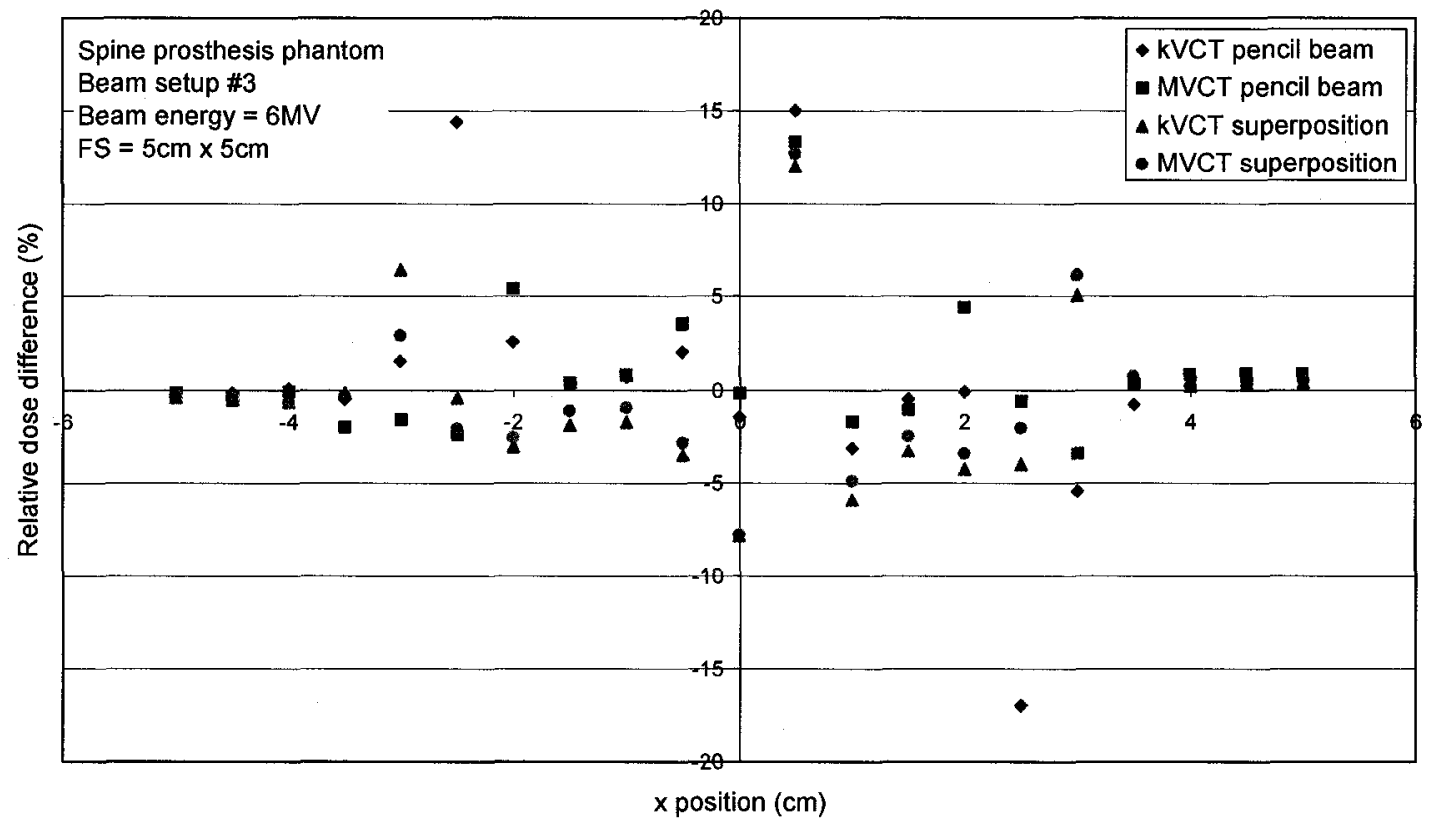

Figure 5.27 The dose difference between treatment planning calculations (pencil beam and superposition algorithms) and MC simulations for a $5 \times 5 \mathrm{~cm}^{2} 6 \mathrm{MV}$ photon beam incident on the central axis (beam setup \#3 in Figure 5.5) of a spinal prosthesis phantom. The depth of the profiles was chosen to intersect the prosthesis as illustrated by the blue dashed line in Figure 5.10. 


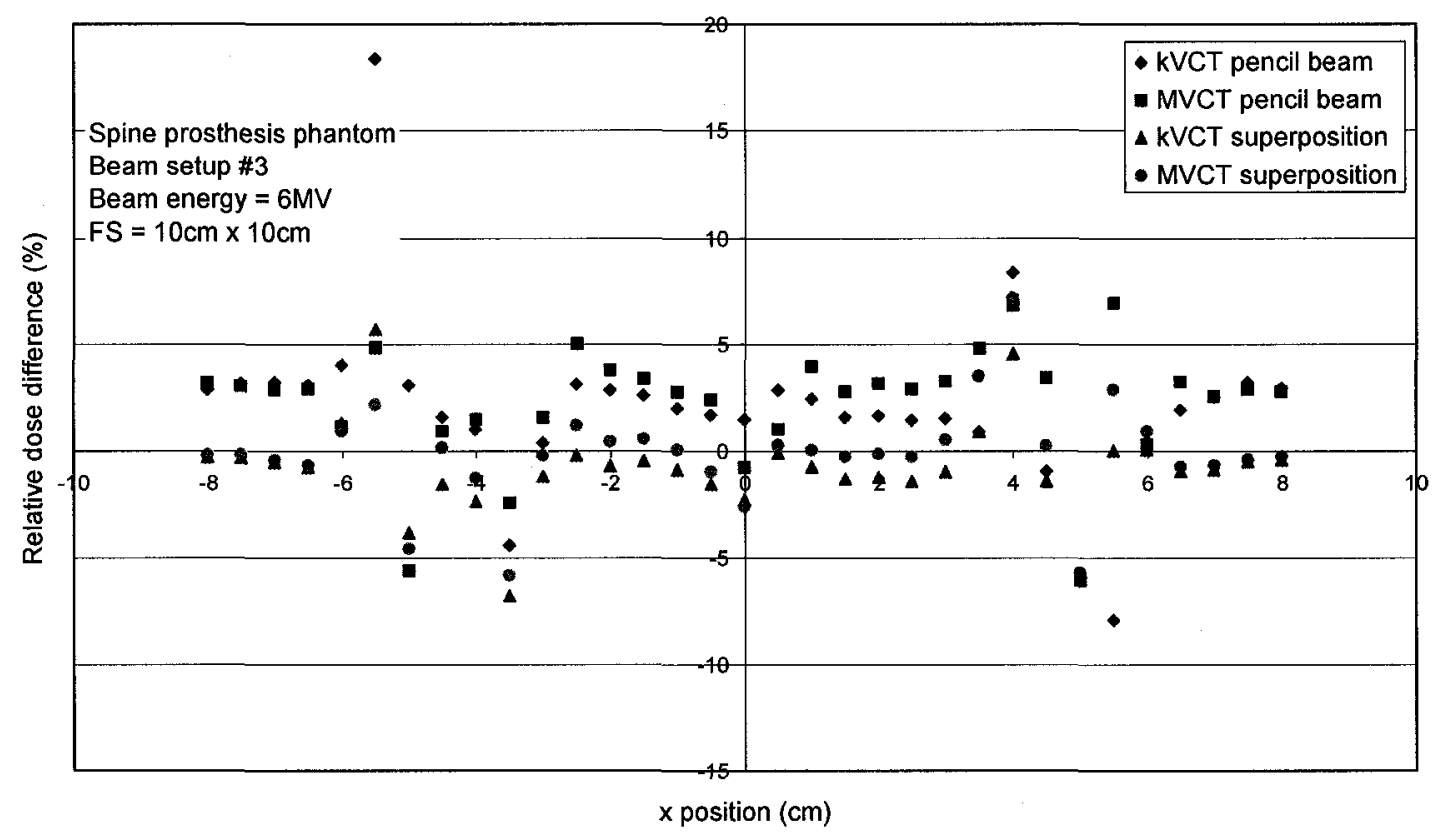

Figure 5.28 The dose difference between treatment planning calculations (pencil beam and superposition algorithms) and MC simulations for a $10 \times 10 \mathrm{~cm}^{2} 6 \mathrm{MV}$ photon beam incident on the central axis (beam setup \#3 in Figure 5.5) of a spinal prosthesis phantom. The depth of the profiles was $2 \mathrm{~cm}$ distal to the spinal rod as illustrated by the red dashed line in Figure 5.10. 


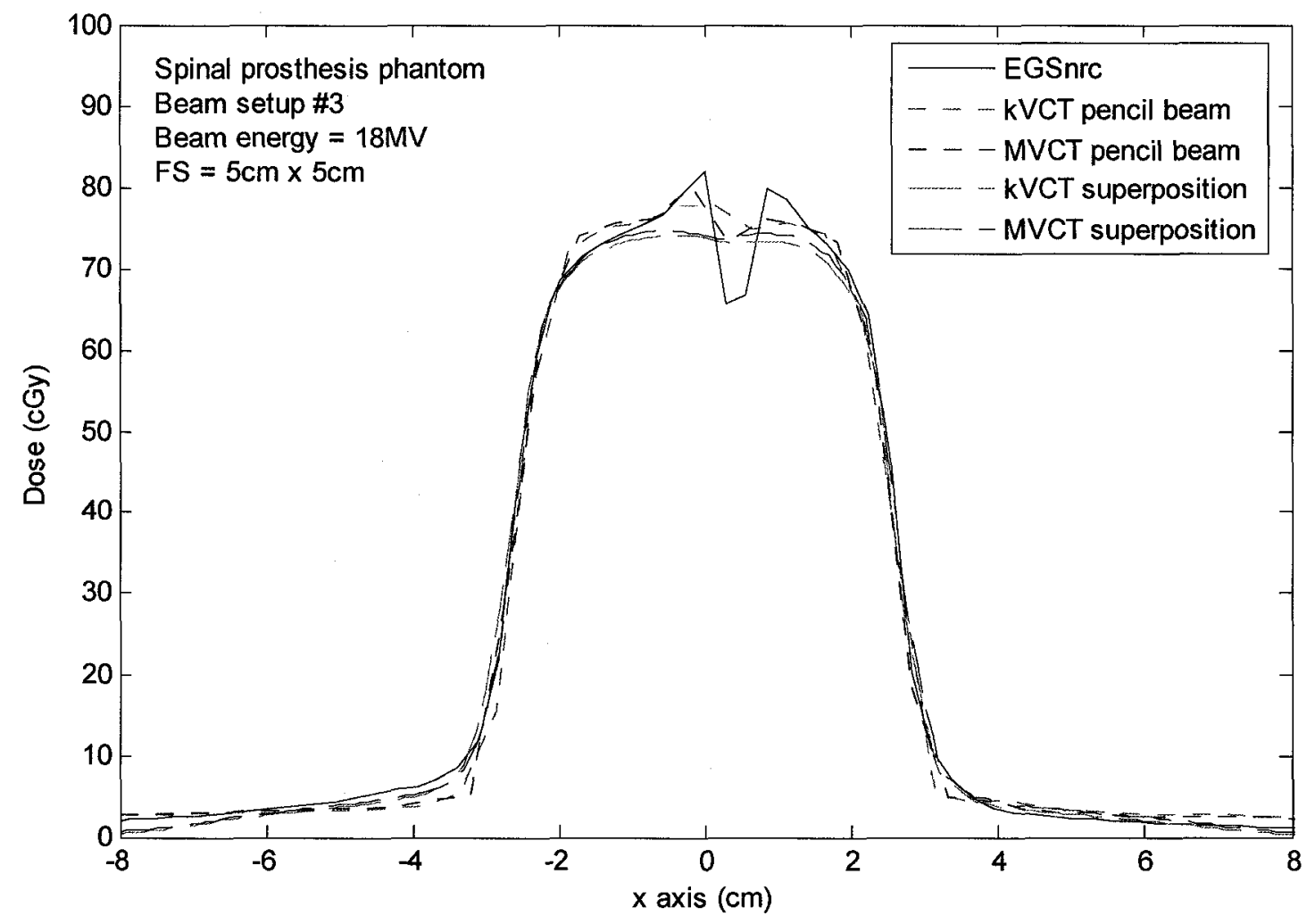

Figure 5.29 kVCT and MVCT based dose profiles calculated with Monte Carlo, pencil beam and superposition algorithms for a $5 \times 5 \mathrm{~cm}^{2} 18 \mathrm{MV}$ photon beam incident on the central axis (beam setup \#3 in Figure 5.5) of a spinal prosthesis phantom. The depth of the profiles was chosen to intersect the prosthesis as illustrated by the blue dashed line in Figure 5.10 . 


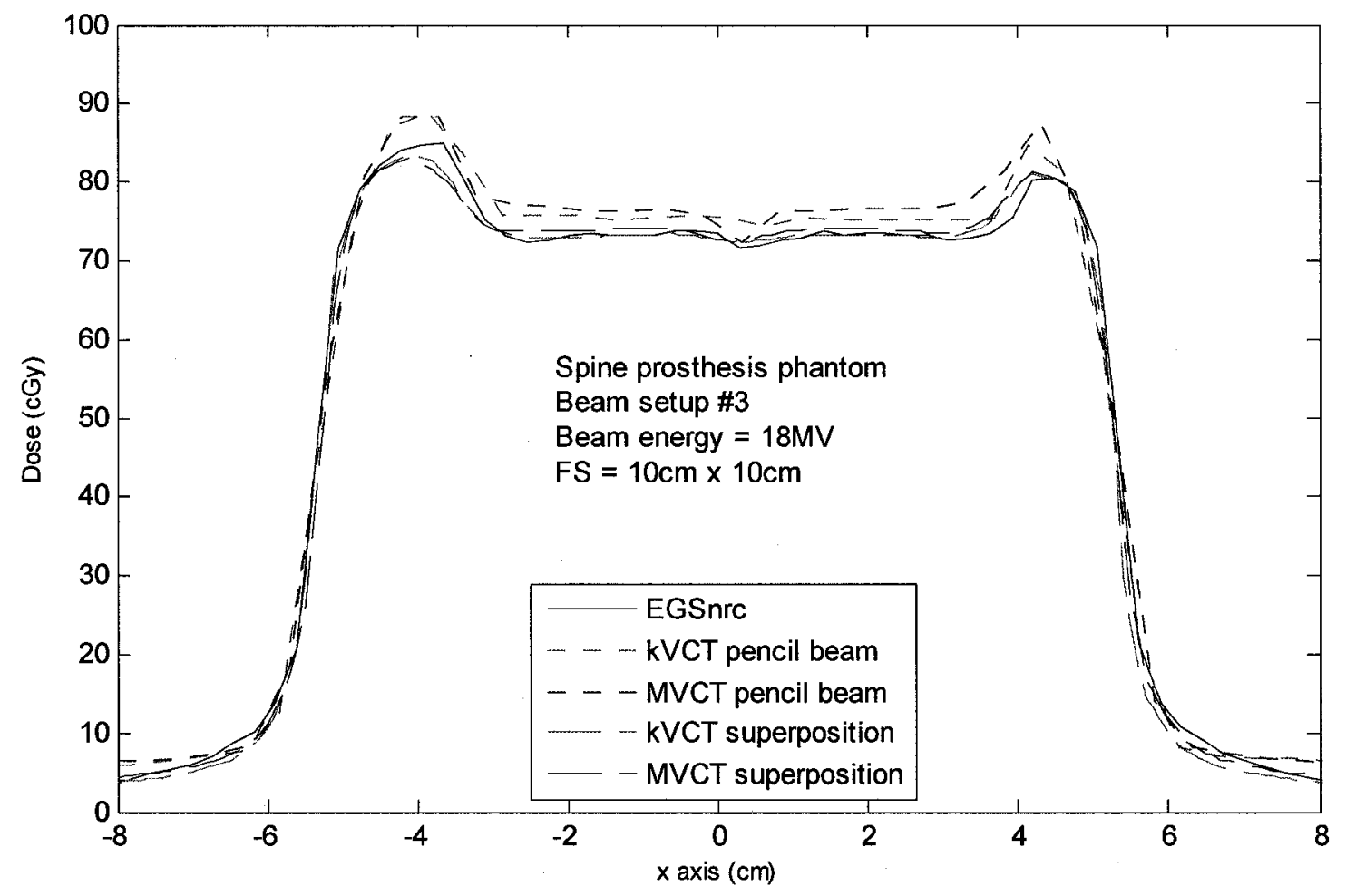

Figure 5.30 kVCT and MVCT based dose profiles calculated with Monte Carlo, pencil beam and superposition algorithms for a $10 \times 10 \mathrm{~cm}^{2} 18 \mathrm{MV}$ photon beam incident on the central axis (beam setup \#3 in Figure 5.5) of a spinal prosthesis phantom. The depth of the profiles was $2 \mathrm{~cm}$ distal to the spinal rod as illustrated by the red dashed line in Figure 5.10 . 


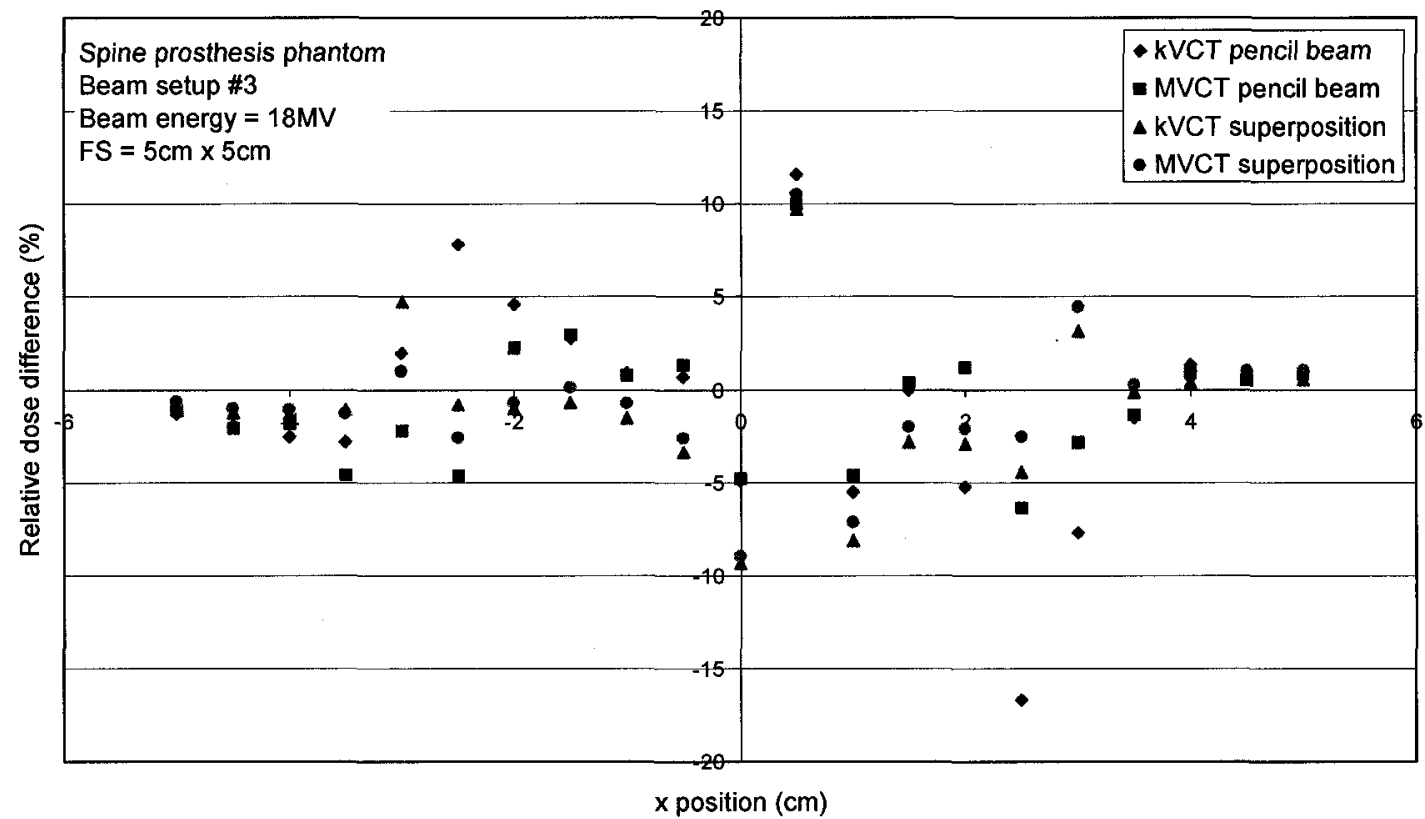

Figure 5.31 The dose difference between treatment planning calculations (pencil beam and superposition algorithms) and MC simulations for a $5 \times 5 \mathrm{~cm}^{2} 18 \mathrm{MV}$ photon beam incident on the central axis (beam setup \#3 in Figure 5.5) of a spinal prosthesis phantom. The depth of the profiles was chosen to intersect the prosthesis as illustrated by the blue dashed line in Figure 5.10. 


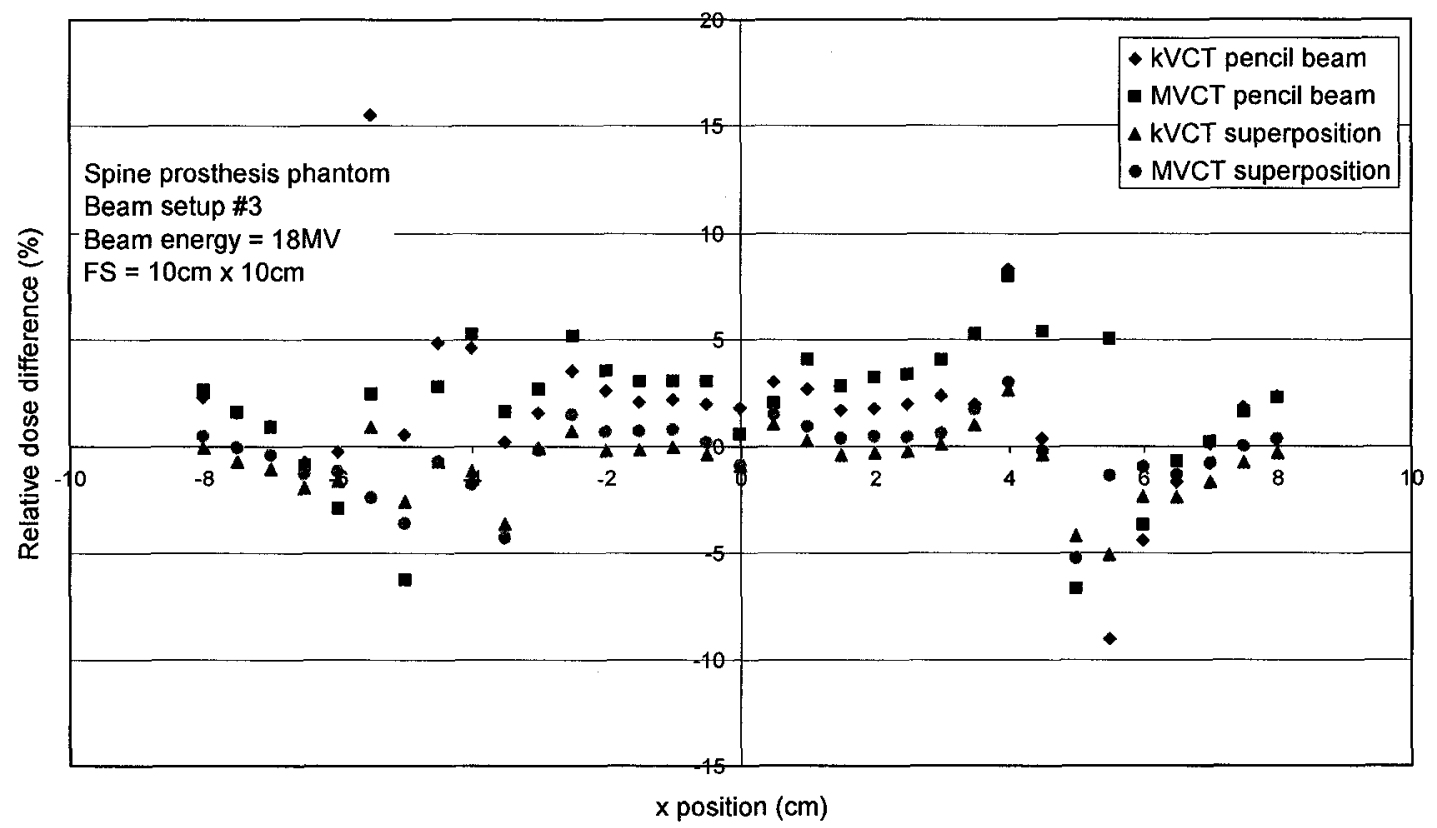

Figure 5.32 The dose difference between treatment planning calculations (pencil beam and superposition algorithms) and MC simulations for a $10 \times 10 \mathrm{~cm}^{2} 18 \mathrm{MV}$ photon beam incident on the central axis (beam setup \#3 in Figure 5.5) of a spinal prosthesis phantom. The depth of the profiles was $2 \mathrm{~cm}$ distal to the spinal rod as illustrated by the red dashed line in Figure 5.10. 


\subsection{Discussion}

It has been well documented that high- $Z$ inhomogeneities irradiated in water phantoms or patients attenuate the radiation beam and cause local perturbations at the water-high- $Z$ interface (Das et al 1990, Das and Khan 1989, Eng et al 2000, Hazuka et al 1988, Hudson et al 1984, Reft et al 2003, Sibata et al 1990 and Webb 1979). These issues can be difficult for current TPSs to model accurately. To complicate matters further, conventional CT scanners with photon energies in the kilo-voltage range produce image artifacts in the presence of high-Z prostheses, potentially making it difficult to delineate critical organs, the tumour or the prosthesis itself. Furthermore, even if the artifacts were minimal, the conversion from $\mathrm{CT}$ number $(\mathrm{HU})$ to electron density may be incorrect. These two sources of error limit the accuracy of the TPS dose calculation since it is dependent upon the accuracy of the relative electron density distribution.

One method of working around the artifacts present in patients with high-Z prostheses is to turn off any inhomogeneity corrections and manually input density in the TPS, though not all commercial TPSs have this capability. The prosthetic device is contoured and any surrounding normal tissue regions with artifacts present would be artificially assigned a CT number that approximately corresponds to water. If the type of prosthesis is known, then the electron density can be determined and assigned to the appropriate region. Alternatively, a megavoltage scan of the prosthesis could give an approximate measurement of the attenuation coefficient and the electron density can then be mapped. This method of contouring the prosthesis has several limitations. Firstly, there are often artifacts immediately surrounding the prosthetic device, making it difficult 
to determine an accurate contour (see Figure 5.3a). Secondly, the CT images will not show whether the prosthesis is hollow since the artifacts will usually fill in these regions. Finally, TPSs may have limitations on the HU or electron density that can be used for inhomogeneity corrections (Reft et al 2003).

Several studies have examined a commercial TPS's ability to model the dose perturbations due to high- $Z$ prostheses by comparing them with Monte Carlo dose calculations. Ding et al (2001) examined the dose distribution surrounding a metal hip prosthesis in a patient and compared this to CADPLAN (TPS) calculations. He reported a dose increase of $15 \%$ at the proximal metal-tissue interface and a $25 \%$ dose reduction in the shadow of a titanium hip prosthesis, similar to what was observed in our study (Figure 5.23). In comparing these results with the TPS, CADPLAN was found to underestimate the attenuation by the prosthesis, resulting in a $14 \%$ overestimation of the target dose for a four-field box beam arrangement. However, it has been shown that these results will vary depending on the dose calculation algorithm, the beam energy and depth. Roberts (2001) investigated the inhomogeneity correction accuracy of the Helax TPS (based on the pencil beam algorithm) for a phantom containing a titanium prosthesis (with a $3 \mathrm{~cm}$ diameter). For $6 \mathrm{MV}$ X-rays and $20 \times 20 \mathrm{~cm}^{2}$ field, the TPS overestimated the beam attenuation by $20 \%$ at $15 \mathrm{~cm}$ and $20 \mathrm{~cm}$ depths $(6.5 \mathrm{~cm}$ and $11.5 \mathrm{~cm}$ beyond the prosthesis). In 2003, AAPM (American Association of Physicists in Medicine) formed the Radiation Therapy Committee Task Group 63 (TG 63) to report on the "Dosimetric considerations for patients with hip prostheses undergoing pelvic irradiation." The dose perturbation results in our work showed broad agreement with the results reviewed in TG 
63 in terms of dose distribution and dose calculation algorithm for phantoms with hip prostheses. Also included within this document were step-by-step recommendations to minimize dose errors in treatment planning and delivery for such patients. Some of the recommendations were to select beam arrangements that avoided the prosthesis, to understand the limitations of your TPS, and to approximate attenuation and possible dose perturbations from knowledge of the prosthesis.

One of the goals of this work was to investigate the benefits of incorporating MVCT-based treatment planning calculations specifically for patients with high-Z prosthetic devices. MVCT-based treatment planning can potentially reduce the two main sources of error (CT reconstruction artifacts and inaccurate conversion from $\mathrm{HU}$ to electron density) that limit the accuracy of conventional kVCT-based treatment planning in the presence of high- $Z$ prostheses: i) reconstruction artifacts and ii) an unreliable conversion of HUs to electron density. This study showed the impact of these deficiencies is not only dependent on the size of the prosthesis but on the treatment planning dose calculation algorithm used.

It is important to note that a comparison between the two algorithms is something that has been well documented and is outside the scope of this study. The focus of this chapter is to highlight the potential improvements when using MVCT over kVCT for specific patients with high-Z prostheses. 
In regions where artifacts were present in the image, the PB algorithm showed an increase or decrease in attenuation of the beam depending on the nature of the artifacts relative to the wax material. This can be due to the lack of lateral electron transport with the PB algorithm. However, superposition methods are known to take into account secondary electron transport and energy deposition from scattered photons (Ahnejo 1989, Mackie 1985, 1988, Papanikalou 1993). For the 6 and $18 \mathrm{MV}$ beams incident on the central axis of the hip phantom (beam setup \#1 in Figure 5.5), the superposition algorithm interpreted the artifacts as a low-density inhomogeneity, resulting in a greater range for secondary electrons and a broader penumbra relative to unit-density tissue material. This is consistent with the results shown in Chapter 3. Interestingly, the accurate modeling of electron transport in the superposition algorithm led to greater dose errors in profile since the low-density regions are due to artifacts and are not actually present in the real phantom (Figures 5.15 and 5.19). Additionally, for this experiment, this also resulted in the PB algorithm showing greater agreement with the Monte Carlo simulations since it did not accurately account for the inhomogeneities.

Figures 5.14 to 5.21 showed that $\mathrm{CT}$ artifacts in regions far from the high-Z material can significantly affect dose calculations even when the beam does not pass through the high-Z material. As previously mentioned, for patients with high-Z hip prostheses, treatment planning CT datasets are often manually altered so that normal tissue regions filled with artifacts are assigned the density of water. However, this is not always a viable solution. For example, head and neck cancer patients with high- $Z$ tooth fillings show huge artifacts in their treatment planning CT images. Figure 5.33 (a) and 
(b) show $\mathrm{kVCT}$ images of a patient with and without high- $\mathrm{Z}$ fillings present in the CT dataset. Assigning all normal tissues the same density as water would result in a loss of information about air cavities. Furthermore, it may be difficult to delineate the teeth, high-Z materials, air cavities and normal tissues in the presence of significant artifacts. The use of MVCT images for treatment planning calculations can aid in the delineation of targets and organs-at-risk. Figure 5.34 shows CT images of a patient with high-Z hip prostheses taken with a conventional kilovoltage CT imager (a) versus an MVCT imager (b). Note the reduction in artifacts in the MVCT image when compared to the kVCT image. For prostate cancer patients, it is critical to define the prostate-rectum interface to achieve acceptable toxicity. MVCT imagers have been shown to have sufficient contrast visibilities for soft tissue and can be used as a solution for delineation in the presence of high-Z materials (Meeks et al 2005, Ruchala et al 2003). 
a)

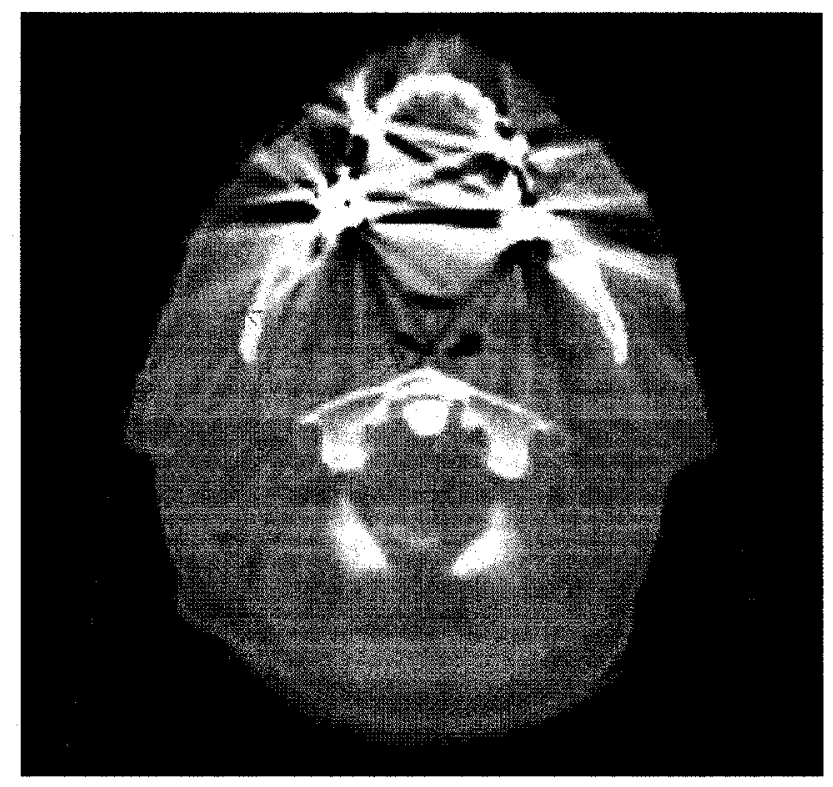

b)

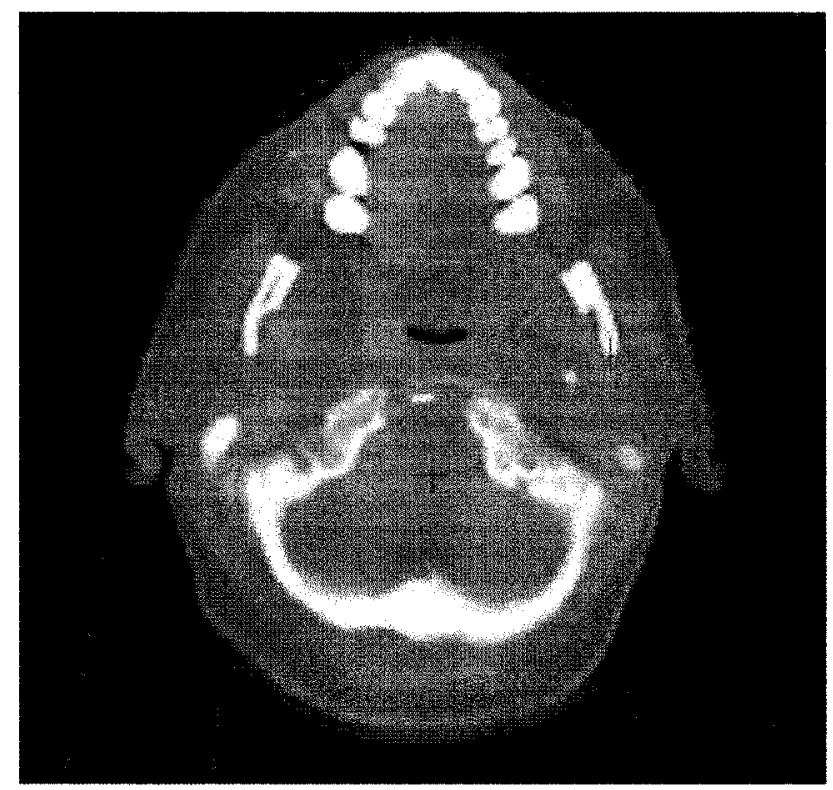

Figure 5.33 Conventional CT images of a patient (a) with and (b) without high-Z fillings present in the oral cavity. 
a)

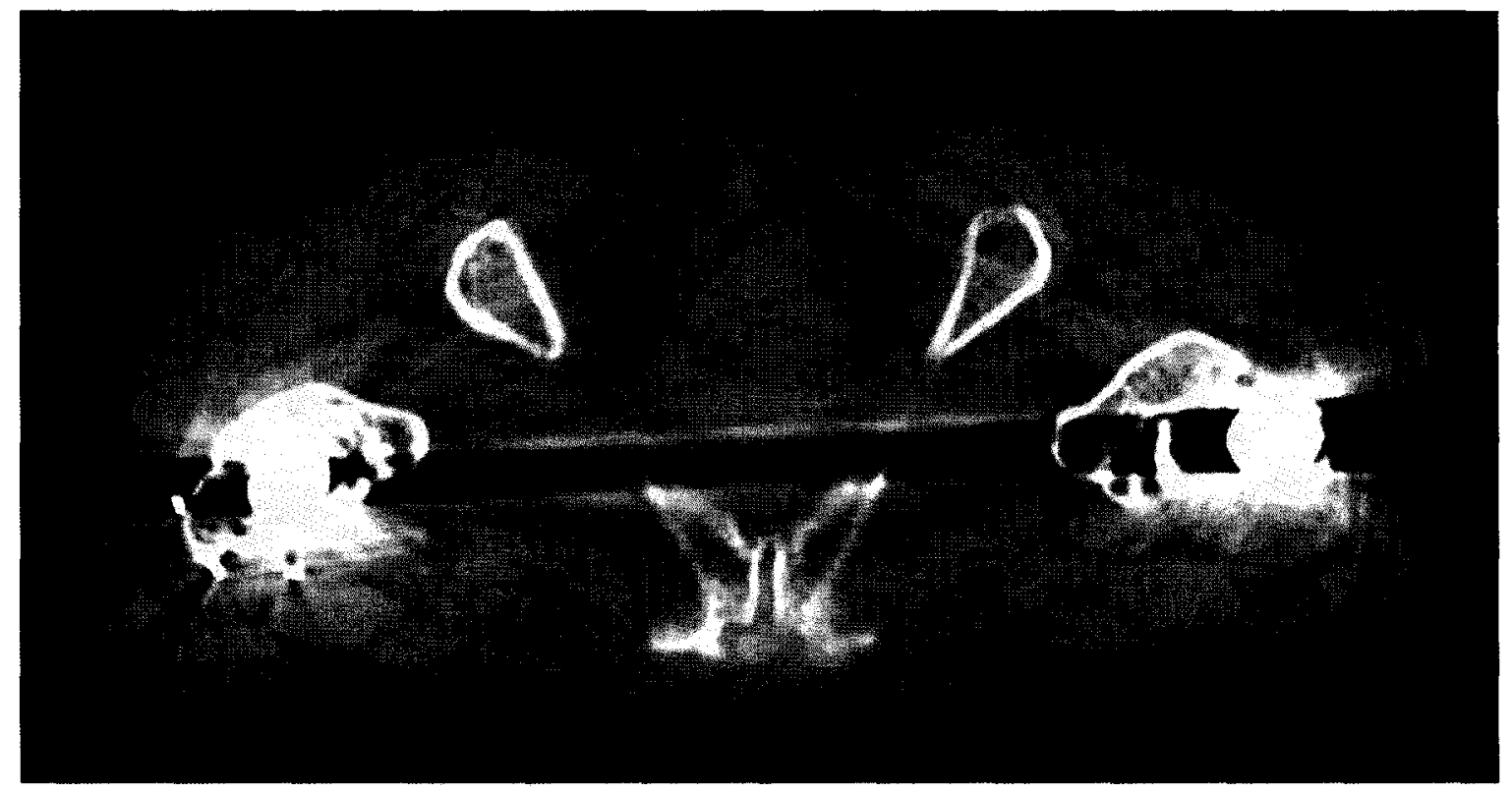

b)

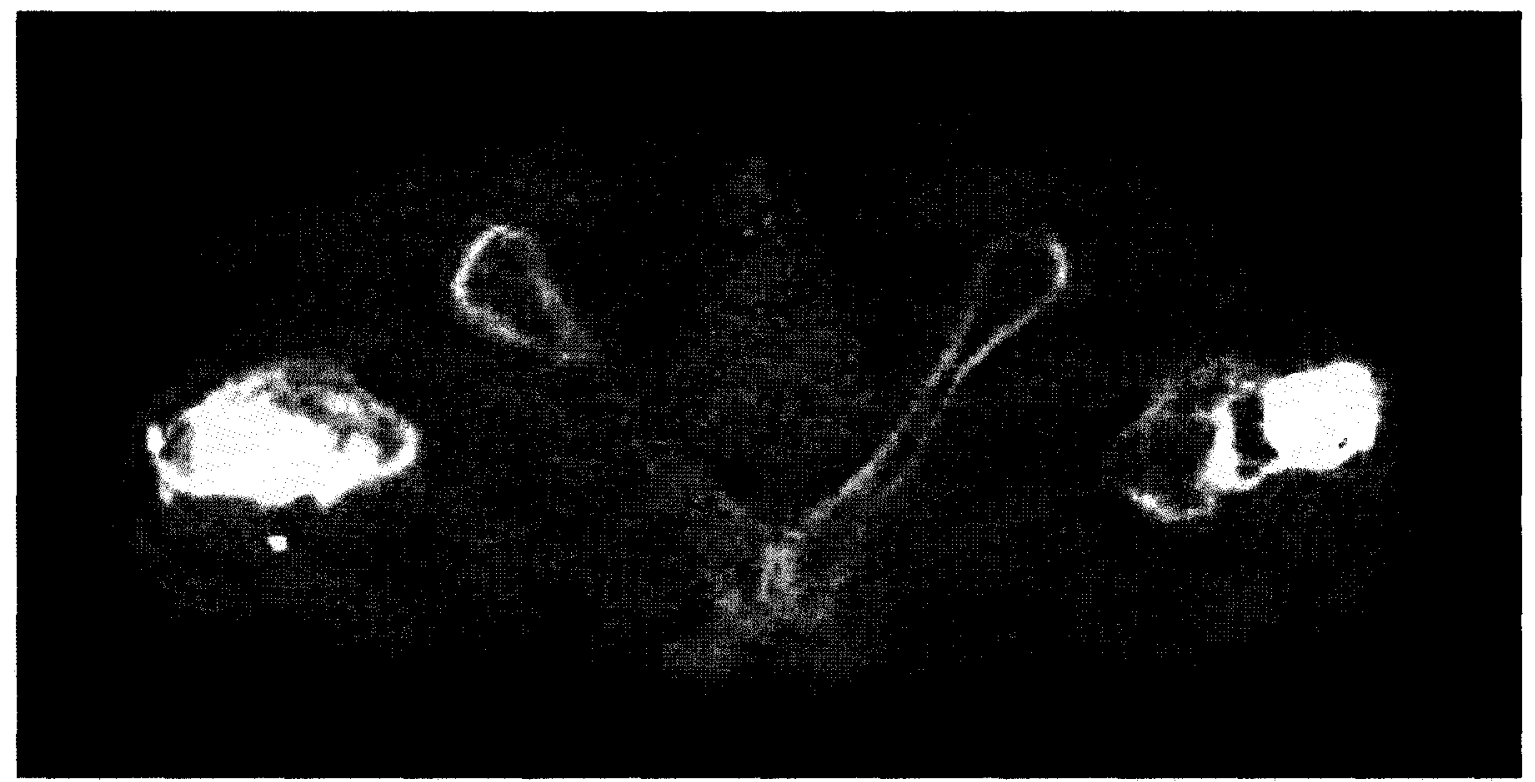

Figure 5.34 CT images of a patient with high-Z hip prostheses taken with (a) kilovoltage and (b) megavoltage X-rays. 
One of the prominent features in the presence of high- $\mathrm{Z}$ inhomogeneities are the dose perturbations at interfaces. These perturbations were present when the hip prosthesis was directly in the center of the geometric beam (beam setup \#2 in Figure 5.5) as shown in Figures 5.22 and 5.23. These are due to an increase in lateral electron scatter from the prosthetic device producing an increased dose in the surrounding wax. Peaks are also visible in the Monte Carlo depth-dose curve, though these are due to multiple factors. The dose perturbation has been shown to be dependent on the incident photon energy, differences in the photon energy transfer coefficients, the atomic number, the mass density and thickness of the inhomogeneity, and the differences in multiple scatter of the secondary electrons (Reft et al 2003). For photon beams greater than 10 $\mathrm{MV}$, the dose distal to the prosthetic device may also have an upwards peak from pair production interactions in the high- $Z$ material. Neither the $\mathrm{PB}$ or superposition algorithms were able to model the lateral electron transport near the interfaces where there is an electronic disequilibrium. Similar trends were also seen when the spinal prosthesis was directly in the center of the geometric beam (beam setup \#3 in Figure 5.5) as shown in Figures 5.25 through 5.32. At these lateral interfaces, the treatment dose will be greater than the predicted dose, potentially leading to greater NTCP than anticipated.

Within the titanium material, the dose difference between the two CT-based calculations can be due to a combination of several factors. Many conventional CT scanners are limited to a maximum Hounsfield number of 3000 . Thus any materials with higher densities and greater attenuation coefficients appear saturated in the kVCT image and are represented by the same Hounsfield unit. Additionally, the actual prosthesis may 
also be hollow, but this will be masked by the artifacts in the kVCT dataset. Finally, the contribution of the photoelectric effect at kilo-voltage $\mathrm{x}$-ray energies in high atomic number materials can result in errors in the mapping of Hounsfield numbers to relative electron density (Brahme et al 1987).

At kVCT energies, photons interact with a combination of Compton and photoelectric processes. Although the Compton interaction coefficient is independent of the dominant interaction process is Compton but as the atomic number of the material increases (such as in bone or high $\mathrm{Z}$ prostheses), there is an increasing contribution from the photoelectric effect. The result is that Hounsfield units are dependent upon both the electron density and the atomic number of the material. At MVCT energies, the dominant interaction process is the Compton process. Therefore, MVCT numbers are expected to be linearly dependent on the electron density of the material, providing a more reliable electron density conversion.

Figure 5.35 compares the kVCT and MVCT Hounsfield number from images (such as those shown in Figure 5.3) of the hip prostheses phantom. The depth of the profiles was chosen to intersect both high $\mathrm{Z}$ devices where a large number of artifacts are present. The depth is illustrated by the blue dashed line in Figure 5.9 (a). The kVCT profile in Figure 5.35 shows that the region between the two prostheses is represented by a HU hovering around -1000 which represents air. Therefore, the kVCT based dose calculations in this region are being based on the electron density of air rather than wax 


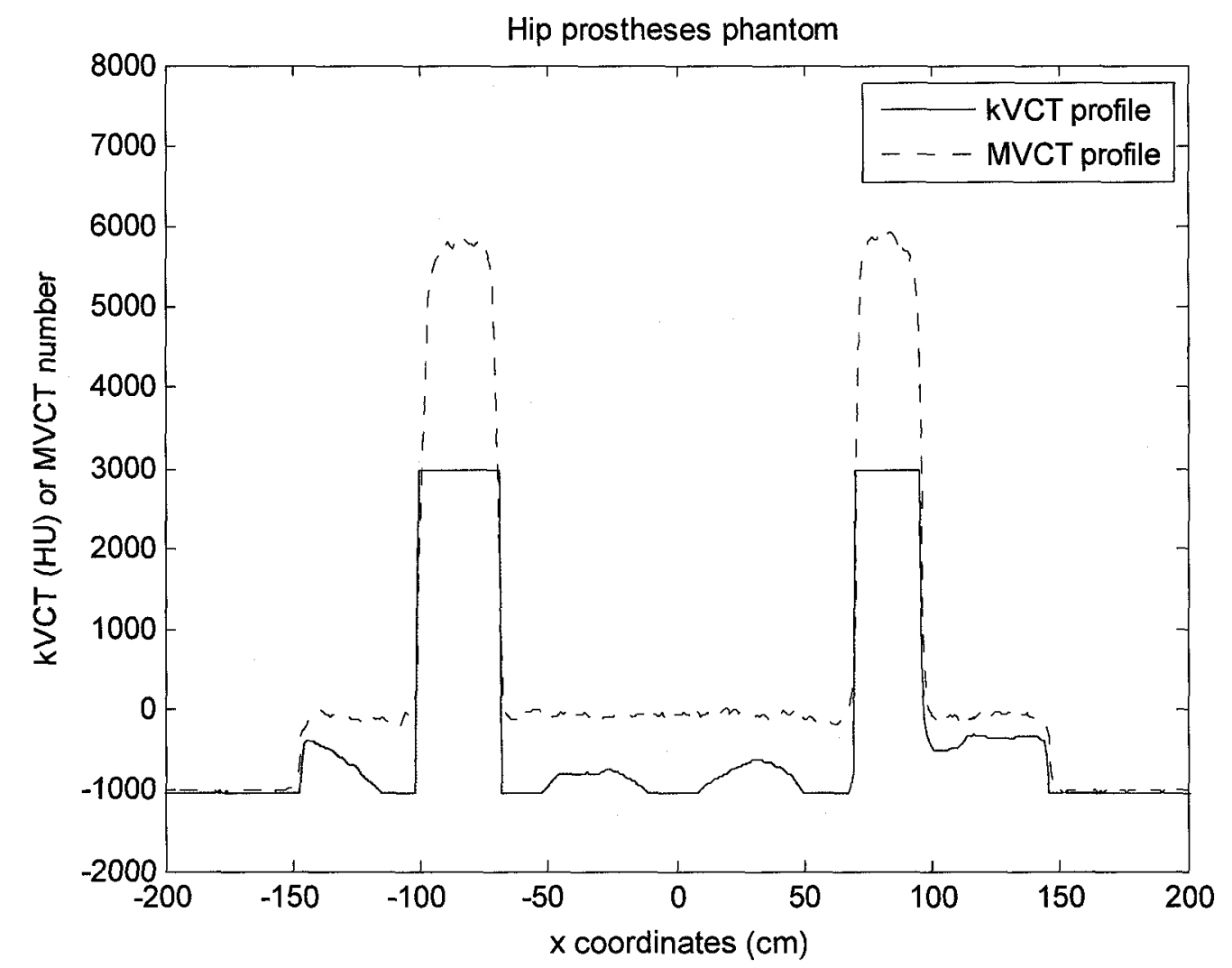

Figure 5.35 kVCT (Hounsfield unit) and MVCT number profiles from images of titanium alloy hip prostheses in a solid wax phantom. The depth of the profile is illustrated (blue dashed line) in Figure 5.7 (a). 


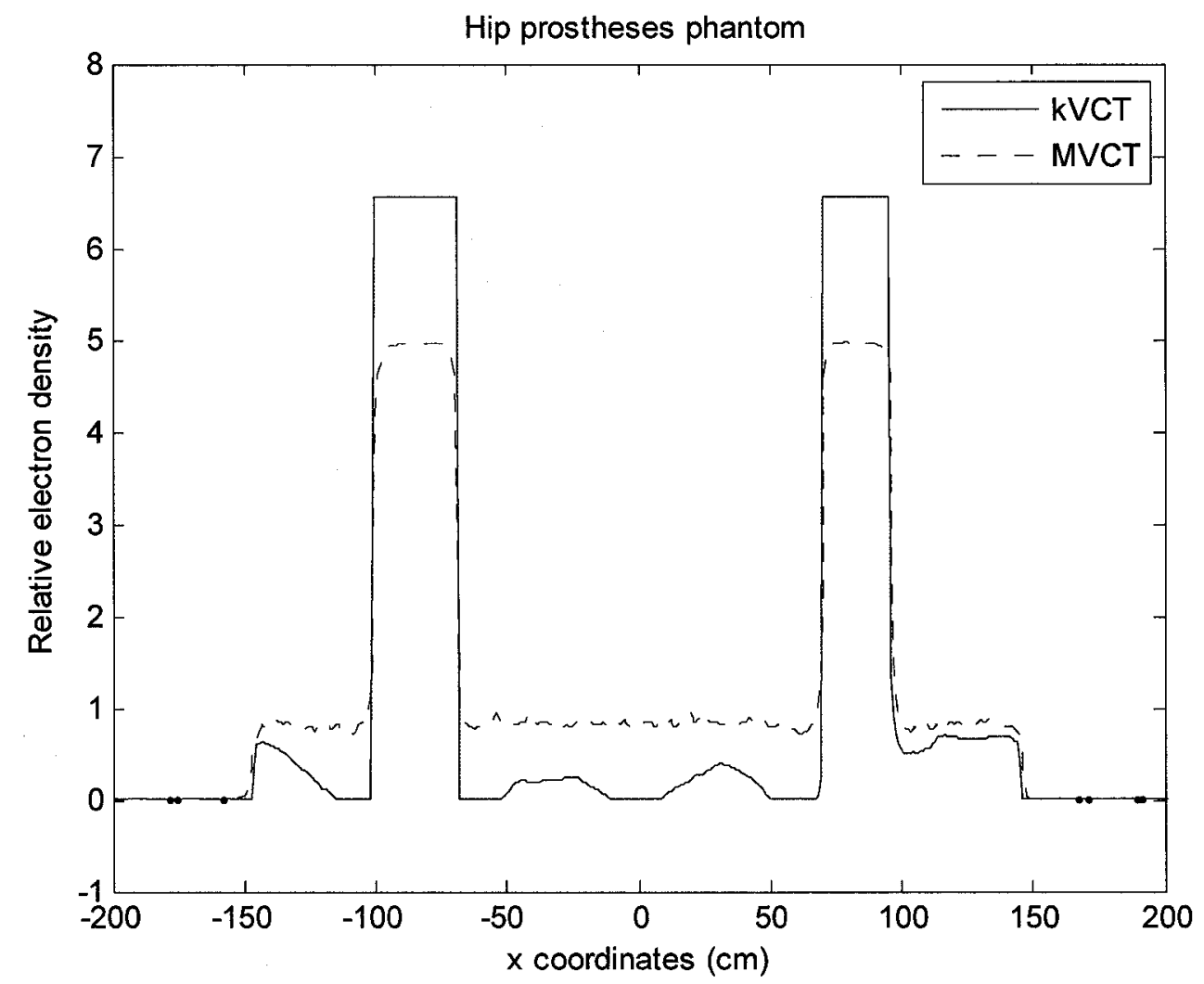

Figure 5.36 Electron density profiles (relative to water) from kVCT and MVCT images (unedited) of titanium alloy hip prostheses in a solid wax phantom. The depth of the profile is illustrated (blue dashed line) in Figure 5.7 (a). 
or water. On the other hand, the MVCT profile represents the wax region relatively uniformly with a CT number just below that of water or normal tissue.

The HU saturation point is also visible in the $\mathrm{kVCT}$ profile, peaking at 3000 within the prosthetic material. However, this does not necessarily result in an underestimation of the relative electron density within the high $\mathrm{Z}$ material. Figure 5.36 shows the corresponding electron density profiles from the KVCT and MVCT images of the hip prostheses phantom. The depth of the profiles is the same as in the CT number profiles. These profiles were calculated by converting the CT number profiles in Figure 5.35 to RED profiles by applying the RED curves shown in Figure 5.6. The theoretical RED of the prostheses is approximately 3.8. However, the KVCT and MVCT profiles show an RED of 6.56 and 4.97, respectively, for the titanium alloy. Therefore, the kVCT system overestimates the RED in the prosthesis by $72 \%$ compared to $31 \%$ by the MVCT system. As previously mentioned in section 5.2.1, the kVCT RED curve peaks at a HU of 6.56 (stainless steel). This highlights a limitation in the RED curve data used in the clinic. More data points are needed between the RED of aluminum (3.12) and stainless steel to get an accurate determination of where the HU saturation point is reached. Otherwise, all materials with a $\mathrm{HU}$ greater than the saturation point will be assigned the RED of stainless steel, as was done in this study.

KVCT image artifacts within the prosthesis masked the partially hollow regions of the ball joint, and instead represented it as a solid sphere of high- $Z$ material. This was represented by the relatively symmetrical "dip" in the kVCT-based superposition 
calculations (Figure 5.7). These hollow regions were more visible in the MVCT images and were modeled accordingly as shown by the asymmetrical "dip" in the MVCT-based superposition profile. The $30 \%$ overestimation of the titanium alloy RED by the MVCT system may also be due to the artifacts present within the prosthesis itself. Though the number and intensity of the MVCT image artifacts were greatly reduced when compared to the $\mathrm{kVCT}$ images, the hollow region within the prosthesis was blurred and possibly increased in intensity. The wax region between the prostheses also showed a slight decrease in CT number.

In summary, the MVCT-based treatment planning calculations generally showed reduced dose errors over kVCT-based treatment planning in the immediate presence of high- $Z$ inhomogeneities. In regions far from the high- $Z$ material, artifacts can significantly affect dose calculations even when the beam does not pass through the high$\mathrm{Z}$ material. Our experiments showed that accurate modeling of electron transport in the superposition algorithm led to greater dose errors in profile since the low-density regions are due to artifacts and are not actually present in the real phantom. Conversely, the PB algorithm showed better agreement with the Monte Carlo simulations since it did not accurately account for the inhomogeneities. When the high- $Z$ prostheses were within the geometric beam, neither CT-based calculations were able to correctly model the peak in the dose profile at lateral prosthesis-wax interfaces. The kVCT and MVCT-based plans underestimated the dose in this region by up to $17 \%$ and $12 \%$, respectively. However, in the wax region beyond this peak, the MVCT-based calculations showed a marked improvement over the kVCT-based calculations. The accuracy of the MVCT HU to 
electron density map and the reduced artifacts over kVCT images are two key reasons for the observed reduction in dose error. For cancer centers with MVCT capability, we feel MVCT-based treatment planning can be a viable option for specific cases of patients with high-Z prosthetic devices. 


\section{Chapter 6}

\section{Conclusions}

The success of radiation therapy treatments depends among other things upon the accuracy of the delivered dose. Once the decision to treat with radiation therapy is made, it is important to understand the many sources of error that can arise during the various preparatory stages that lead to dose delivery. It is recommended that the cumulative dose error throughout the entire treatment be limited to +7 and $-5 \%$ of the prescribed dose (ICRU 50 and 62). In this thesis, we examined specific sources of dose error that can arise during the treatment planning stages of radiotherapy. In these stages, a treatment planning CT of the patient is acquired and sent to the TPS where the HU representation of the patient is mapped to relative electron density. The TPS utilizes a dose calculation algorithm to predict the dose distribution in the patient based on the electron density distribution determined from the HU map. 
The sources of error investigated in this work can be categorized as:

i) Errors in the HU distribution representing the patient

ii) Errors in the mapping of the HU into electron density (or physical density)

iii) Errors in the dose calculation algorithm.

In chapter 3 the focus is on type iii) errors in investigating the Theraplan Plus v3.8 (with Oncentra DCM version 2.0) implementation of the PB and CCC dose calculation algorithms and their performance in lung-equivalent material. These algorithms have been extensively investigated in various commercial TPSs, but to my knowledge, have not yet been examined for the Theraplan implementation. As expected, the largest dose errors were observed near the lateral lung-acrylic interfaces where lateral disequilibrium exists. The pencil beam algorithm was not able to model the broadening of the penumbra due to lateral electron transport in the lung-equivalent material. The $\mathrm{CCC}$ algorithm also had difficulty with this as well, but showed an improvement over the pencil beam algorithm. The magnitude of the dose errors were in agreement with published material. In general, the $\mathrm{CCC}$ algorithm consistently showed smaller dose errors than the pencil beam algorithm.

Chapter 4 examined type i) dose errors by looking at the errors which arise because of objects being present during treatment that are not present in the treatment planning CT scan and hence not accounted for in the treatment plan. Specifically we looked at the attenuation of photon beams by a carbon fiber treatment tabletop. Ultimately, this is an error in the $\mathrm{HU}$ representation of the patient. We determined that 
neglecting the attenuation of oblique treatment fields by the carbon fiber couch can result in localized dose reduction from $4 \%$ to $16 \%$, depending on energy, field size, and geometry. Some manufacturers of treatment tabletops have responded to the concern regarding attenuation of the beam by the tabletop by creating removable side rails, the portion of the table producing the greatest attenuation. Alternatively, this study shows that incorporating the carbon fiber couch in the TPS patient model reduces the dose error to less than 2\% (using Theraplan Plus v3.8). From this study, the routine incorporation of the treatment tabletop into patient treatment planning dose calculations is proposed. It should be noted that this requires stringent patient positioning, particularly laterally on the couch and it is suggested that such corrections only be used when table indexing and patient immobilization are used clinically.

In chapter 5 , both type i) and ii) errors were examined. This work investigated treatment planning dose errors in the presence of high- $\mathrm{Z}$ prostheses and compared TPS calculations based on KVCT with MVCT. The two main sources of error in this study were i) reconstruction streak artifacts in the kVCT images and ii) an incorrect mapping from the $\mathrm{CT}$ number $(\mathrm{HU})$ to the relative electron density. The first source represents an error in the HU representation of the patient while the second represents an error in the electron density distribution of the patient. The results showed consistently more accurate dose calculations (for both pencil beam and superposition algorithms) when using MVCT over $\mathrm{kVCT}$ in the presence of high-Z prostheses. The reconstruction artifacts were greatly reduced with the use of MVCT, giving a much more accurate $\mathrm{HU}$ representation of the patient. It would also potentially allow for the clear delineation of 
surrounding critical organs and tumours. MVCT images also improved the reliability of the electron density map. In normal tissue, conventional $\mathrm{kV}$ X-rays interact primarily via Compton scatter but in higher- $Z$ materials, the photoelectric effect contributes significantly. Thus for materials with a greater density than bone, various materials with different atomic numbers but equal electron density can be represented by the same kVCT Hounsfield unit. For photons in the megavoltage range, the contribution from Compton scatter is still nearly independent of $\mathrm{Z}$ and the photoelectric interaction coefficient is negligible. This leads to a more reliable map of the electron density from the HU data. For clinics that have MVCT capability, it is recommend that MVCT-based treatment planning is an improved solution over previous methods involving modification of the beam geometry to avoid the prosthesis or manual editing of the CT data. Adjusting the beam geometry around the prosthesis can lead to increased doses to critical structures while manual adjustment of the CT dataset is limited by the visibility of the prosthesis in the images and requires several approximations.

\subsection{Future Work}

At the time the work in chapter 3 was performed, the pencil beam algorithm implemented by Theraplan Plus was the TPS at the Ottawa Hospital Cancer Centre (OHCC). As the years have progressed, many clinics including $\mathrm{OHCC}$ have upgraded to more accurate algorithms on newer TPSs. However, for clinics using this version of Theraplan it is still important to understand the limitations of the TPS and the clinical impact of these limitations. It can be interesting to propagate the dose errors into an outcome analysis to investigate correlation to tumour control probability and/or complication rates. This 
could help determine whether adjustments to the treatment plan are necessary or whether it is cost-effective to invest in a new TPS.

Since this study in chapter 4 was performed, the carbon fiber tabletop grid region was found to sag and was replaced by a firmer, thicker and more solid carbon fiber insert. It is believed that this would only increase the potential dose error due to attenuation of the beam. Furthermore, there is concern of an increased skin dose for posterior beams since the table insert would be a source of electrons and decrease the skin sparing effect of photon beams. It has been shown that table inserts and immobilization devices can increase surface doses from $21 \%$ to $55 \%$ for a $6 \mathrm{MV}$ photon beam (de Ost et al 1997) and the magnitude of this increase is larger for smaller field sizes (Higgins et al 2001). Field sizes in conformal therapies are getting smaller and smaller with the inception of techniques such as IMRT and TomoTherapy. Future work could investigate increased skin dose and attenuation from the tabletop, inserts and immobilization devices for more complex treatments and for table tops from other manufacturers.

The number of patients with prosthetic devices that could affect their radiation treatments was found to be up to $4 \%$ (Reft et al 2003). A common treatment site that incorporates hip prostheses is the prostate. A potential direction for this work could be to incorporate patient data into this study and examine the impact of introducing MVCTbased TPS calculations. A dose-volume histogram analysis could be performed to investigate the increased or decreased dose to the tumour as well as to organs at risk such as the bladder and rectum. Another treatment site of interest would be the oral cavity. 
Patients with silver amalgam fillings that are being treated for oral cancers with radiation therapy may also benefit from MVCT-based planning.

Another interesting challenge would be to address the difference between electron and mass density in TPSs, as briefly discussed in Chapter 5. Treatment planning systems make the assumption that all materials are water of variable density. This holds relatively true for materials with a density of water or less. However, for higher-Z materials, Figure 5.7 showed that the difference between the mass and electron densities increased significantly. One could examine the impact of this difference on patient dose and investigate methods of incorporating mass density into treatment planning systems. $\mathrm{MC}$ based dose calculation algorithms incorporate mass density but further work could be done to extend this to treatment planning systems based on semi-analytic algorithms. 


\section{Appendix A}

\section{Thermo-luminescent dosimeter (TLD) reproducibility statistics}


Table A.1 Accuracy and reproducibility of TLD chips (Box 3 and 4) irradiated with 100 cGy. The data was derived from 3 consecutive calibration experiments. The values represent individual TLD calibration factors and their respective error within a $95 \%$ confidence interval.

\begin{tabular}{|c|c|c|c|c|c|c|c|c|c|c|c|c|c|}
\hline \multirow{2}{*}{$\begin{array}{c}\frac{\text { Dosimeter }}{\underline{\text { ID }}} \\
\text { A1 }\end{array}$} & \multicolumn{3}{|c|}{$\frac{\text { Box } 3 \text { TLD's }}{\text { Avg Cal Dose }}$} & \multicolumn{3}{|c|}{$\frac{\text { Box } 4 \text { TLD's }}{\text { Avg Cal Dose }}$} & \multirow{2}{*}{$\begin{array}{c}\frac{\text { Dosimeter }}{\text { ID }} \\
\text { E1 }\end{array}$} & \multicolumn{3}{|c|}{$\frac{\frac{\text { Box } 3 \text { TLD's }}{\text { Avg Cal Dose }}}{\text { (cGy) }}$} & \multicolumn{3}{|c|}{$\frac{\text { Box } 4 \text { TLD's }}{\frac{\text { Avg Cal Dose }}{\text { (cGy) }}}$} \\
\hline & 86.6 & \pm & 0.8 & 102.4 & \pm & 0.6 & & 89.2 & \pm & 0.2 & 95.7 & \pm & 0.6 \\
\hline $\mathrm{A} 2$ & 93.3 & \pm & 0.3 & 91.3 & \pm & 0.7 & E2 & 91.7 & \pm & 1.5 & 88.3 & \pm & 0.7 \\
\hline $\mathrm{A} 3$ & 88.3 & \pm & 0.9 & 89.9 & \pm & 0.9 & E3 & 89.5 & \pm & 1.1 & 96.6 & \pm & 0.8 \\
\hline A4 & 89.3 & \pm & 1.8 & 89.1 & \pm & 0.8 & E4 & 87.6 & \pm & 1.4 & 91.6 & \pm & 1.0 \\
\hline A5 & 94.6 & \pm & 0.9 & 101.8 & \pm & 0.9 & E5 & 93.4 & \pm & 0.6 & 95.8 & \pm & 0.5 \\
\hline$A 6$ & 88.8 & \pm & 0.9 & 87.7 & \pm & 0.6 & E6 & 89.5 & \pm & 1.4 & 97.4 & \pm & 1.8 \\
\hline B1 & 99.5 & \pm & 1.3 & 96.3 & \pm & 1.0 & F1 & 87.0 & \pm & 0.9 & 100.4 & \pm & 0.9 \\
\hline B2 & 88.1 & \pm & 1.4 & 99.7 & \pm & 0.9 & $\mathrm{~F} 2$ & 89.8 & \pm & 0.8 & 97.5 & \pm & 0.7 \\
\hline B3 & 92.3 & \pm & 0.8 & 92.8 & \pm & 1.2 & F3 & 88.8 & \pm & 1.2 & 99.5 & \pm & 0.2 \\
\hline B4 & 97.8 & \pm & 1.2 & 91.3 & \pm & 1.3 & $\mathrm{~F} 4$ & 89.3 & \pm & 0.5 & 92.9 & \pm & 1.0 \\
\hline B5 & 94.3 & \pm & 1.1 & 91.1 & \pm & 0.5 & F5 & 94.2 & \pm & 0.2 & 98.2 & \pm & 1.0 \\
\hline B6 & 91.3 & \pm & 1.3 & 95.9 & \pm & 1.1 & F6 & 91.7 & \pm & 0.5 & 92.7 & \pm & 0.1 \\
\hline C1 & 91.2 & \pm & 0.8 & 89.3 & \pm & 1.0 & G1 & 86.6 & \pm & 1.7 & 98.9 & \pm & 0.2 \\
\hline $\mathrm{C} 2$ & 87.7 & \pm & 1.2 & 100.3 & \pm & 2.0 & $\mathrm{G} 2$ & 82.8 & \pm & 0.9 & 89.8 & \pm & 0.8 \\
\hline C3 & 91.0 & \pm & 1.7 & 90.6 & \pm & 0.7 & G3 & 81.4 & \pm & 0.7 & 97.1 & \pm & 0.5 \\
\hline C4 & 93.1 & \pm & 0.9 & 90.6 & \pm & 1.2 & G4 & 95.2 & \pm & 1.9 & 95.3 & \pm & 0.4 \\
\hline C5 & 93.9 & \pm & 1.1 & 93.1 & \pm & 0.5 & G5 & 94.0 & \pm & 1.5 & 87.8 & \pm & 0.9 \\
\hline C6 & 88.6 & \pm & 1.7 & 89.5 & \pm & 1.0 & G6 & 94.8 & \pm & 1.0 & 97.5 & \pm & 0.4 \\
\hline D1 & 92.8 & \pm & 0.9 & 96.2 & \pm & 1.8 & $\mathrm{H} 1$ & 85.8 & \pm & 0.7 & 94.7 & \pm & 0.9 \\
\hline D2 & 92.7 & \pm & 0.3 & 93.1 & \pm & 0.5 & $\mathrm{H} 2$ & 83.2 & \pm & 0.6 & 101.3 & \pm & 1.0 \\
\hline D3 & 90.1 & \pm & 1.1 & 91.6 & \pm & 1.0 & $\mathrm{H} 3$ & 96.4 & \pm & 1.7 & 98.3 & \pm & 0.6 \\
\hline D4 & 95.8 & \pm & 1.6 & 95.0 & \pm & 1.8 & $\mathrm{H} 4$ & 83.7 & \pm & 0.2 & 90.1 & \pm & 0.7 \\
\hline D5 & 90.7 & \pm & 0.9 & 93.4 & \pm & 2.5 & H5 & 90.8 & \pm & 1.8 & 99.4 & \pm & 0.6 \\
\hline D6 & 90.9 & \pm & 1.3 & 99.0 & \pm & 1.4 & $\mathrm{H} 6$ & 90.5 & \pm & 1.0 & 94.1 & \pm & 0.3 \\
\hline
\end{tabular}




\section{References}

Advantages of multiple algorithm support in treatment planning system for external beam dose calculations. J Can Res Ther, 1, 12-20 (2005).

Ahnesjö A and Aspradakis MM. Dose calculations for external photon beams in radiotherapy. Phys Med Biol, 44, R99-R155 (1999).

Ahnesjö A and Mackie TR. Analytical description of Monte Carlo generated photon dose convolution kernels, Int Conf on the Use of Computers in Radiation Therapy, $L X$ ICCR (Scheveningen, The Netherlands), ed Bruinvis AD, van der Giessen PH, van Kleffens HJ and Wittkämper FW (Amsterdam: Elsevier) pp197-200 (1987).

Ahnesjö A, Andreo P and Brahme A. Calculation and application of point spread functions for treatment planning with high-energy photon beams. Acta Oncol, 26, 49-56 (1987).

Ahnesjö A, Saxner $M$ and Trepp A. A pencil beam model for photon dose calculation. Med Phys, 19, 263-273 (1992).

Ahnesjö A. Collapsed cone convolution of radiant energy for photon dose calculation in heterogeneous media. Med Phys, 16, 577-592 (1989).

Alam R, Ibbott GS, Rourang R and Nath R. Application of AAPM Radiation Therapy Committee Task Group 23 test package for comparison of two treatment planning systems for photon external beam radiotherapy. Med Phys, 24, 2043-2054 (1997).

Allen AM, Czerminska M, Jänne PA, Sugarbaker DJ, Bueno R, Harris JR, Court L and Baldini EH. Fatal pneumonitis associated with intensity-modulated radiation therapy for mesothelioma. Int J Radiat Oncol Biol Phys, 65, 640-645 (2006).

Andreo $\mathrm{P}$ and Brahme A. Stopping power data for high-energy photon beams. Phys Med Biol, 31, 839-58 (1986).

Andreo $\mathrm{P}$ and Nahum AE. Stopping power ratio for a photon spectrum as a weighted sum of the values for monoenergetic photon beams. Phys Med Biol, 30, 1055-65 (1985).

Andreo P. Stopping-power ratios for dosimetry. Monte Carlo Transport of Electrons and Photons, ed Jenkins TM, Nelson WR and Rindi A. New York: Plenum, 485-501 (1988). 
Arnfield MR, Siantar CH, Siebers J, Garmon P, Cox L and Mohan R. The impact of electron transport on the accuracy of computed dose. Med Phys, 27, 1266-1274 (2000).

Aspradakis MM, Morrison RH, Richmond ND and Steele A. Experimental verification of convolution / superposition photon dose calculations for radiotherapy treatment planning. Phys Med Biol, 48, 2873-2893 (2003).

Attix F. Introduction to Radiological Physics and Radiation Dosimetry. New York: Wiley (1986).

Aubin M, Morin O, Chen J, Gillis A, Pickett B, Aubry JF, Akazawa C, Speight J, Roach $\mathrm{M} 3^{\text {rd }}$ and Pouliot $\mathrm{J}$. The use of megavoltage cone-beam CT to complement CT for target definition in pelvic radiotherapy in the presence of hip replacement. $\mathrm{Br}$ J Radiol, 79, 918-921 (2006).

Beach JL, Mendiondo MS and Mendiondo OA. A comparison of air-cavity inhomogeneity effects for cobalt-60, 6-, and 10-MV x-ray beams. Med Phys, 14, 140-144 (1987).

Behrens CF. Dose build-up behind air cavities for Co60, 4, 6 and 8 MV. Measurements and Monte Carlo simulations. Phys Med Biol, 51, 5937-5950 (2006).

Blomquist $\mathrm{M}$ and Karlsson M. Measured lung dose correction factors for $50 \mathrm{MV}$ photons. Phys Med Biol, 43, 3225-3234 (1998).

Boyer AL and Mok EC. A photon dose distribution model employing convolution calculations. Med Phys, 12, 169-177 (1985).

Boyer AL and Schultheiss T. Effects of dosimetric and clinical uncertainty on complication-free local tumor control. Radiother Oncol, 11, 65-71 (1988).

Brahme A, Chavaudra J, McCullough E, Nüsslin F, Rawlinson A, Svensson G, Landberg $\mathrm{T}$, Rossi $\mathrm{H}$ and Wambersie $\mathrm{A}$. Accuracy requirements and quality assurance of external beam therapy with photons and electrons. Acta Oncologica, Suppl.\#1 (1988).

Butson MJ, Cheung T, Yu PKN and Webb B. Variations in skin dose associated with linac bed material at 6 MV x-ray energy. Phys Med Biol, 47, N25-N30 (2002)

Butson MJ, Elferink R, Cheung T, Yu PKN, Stokes M, Quach KY and Metcalfe P. Verification of lung dose in an anthropomorphic phantom calculated by the collapsed cone convolution method. Phys Med Biol, 45, N143-N149 (2000).

Canadian Cancer Statistics 2006, Canadian Cancer Society / National Cancer Institute of Canada, Toronto, Canada (2006). 
CAPCA (Canadian Association of Provincial Cancer Agencies), Standards for Quality Control at Canadian Radiation Treatment Centres: Medical Linear Accelerators.

Carl $\mathrm{J}$ and Vestergaard A. Skin damage probabilities using fixation materials in highenergy photon beams. Radiother Oncol, 55, 191-198 (2000).

Carrasco P, Jornet N, Duch MA, Weber L, Ginjaume M, Eudaldo T, Jurado D, Ruiz A and Ribas M. Comparison of dose calculation algorithms in phantoms with lung equivalent heterogeneities under conditions of lateral electronic disequilibrium. Med Phys, 31, 2899-2911 (2004).

Chow JCL, Wong E, Chen IZ and Van Dyk J. Comparison of dose calculation algorithms with Monte Carlo methods for photon arcs. Med Phys, 30, 2686-2694 (2003).

Cranmer-Sargison G, Beckham WA and Popescu IA. Modelling an extreme waterlung interface using a single pencil beam algorithm and the Monte Carlo method. Phys Med Biol, 49, 1557-1567 (2004).

Cunningham JR. Scatter-air ratios. Phys Med Biol, 17, 42-51 (1972).

Das IJ and Chopra KL. Backscatter dose perturbation in kilovoltage X-ray photon beams at high atomic number interfaces. Med Phys, 22, 767-773 (1995).

Das IJ, Kase KR, Meigooni AS, Khan FM and Werner BL. Validity of transition-zoe dosimetry at high atomic number interfaces in megavoltage photon beams. Med Phys, 17, 10-16 (1990).

Das IJ and Khan FM. Backscatter dose perturbation at high atomic number interfaces in megavoltage photon beams. Med Phys, 16, 367-375 (1989).

de Mooy LG. The use of carbon fibers in radiotherapy. Radiother Oncol, 22, 140-142 (1991).

de Ost B, Vanregemorter J, Schaeken B and Van den Weyngaert D. The effect of carbon fiber inserts on the build-up and attenuation of high energy photon beams. Radiother Oncol, 45, 275-277 (1997).

Deng J, Guerrero T, Ma C-M and Nath R. Modelling 6MV photon beams of a sterotactic radiosurgery system for Monte Carlo treatment planning. Phys Med Biol, 49, 1689-1704 (2004).

Ding GX. Energy spectra, angular spread, fluence profiles and dose distributions of 6 and $18 \mathrm{MV}$ photons beams: results of Monte Carlo simulations for a Varian 2100EX accelerator. Phys Med Biol, 47, 1025-1046 (2002). 
Ding GX and Yu CW. A study on beams passing through hip-prosthesis for pelvic radiation treatment. Int J Radiat Onc Biol Phys, 51, 1167-1175 (2001).

Dutreix A. When and how can we improve precision in radiotherapy? Radiother Oncol, 2, 275-292 (1984).

El-Khatib E and Battista JJ. Accuracy of lung dose calculations for large-field irradiation with 6-MV x rays. Med Phys, 13, 111-116 (1986).

El-Khatib E and Battista JJ. Improved lung dose calculation using tissue-maximum ratios in the Batho correction. Med Phys, 11, 279-286 (1984).

Eng TY. Dose attenuation through a titanium alloy hip prosthesis. Med Dosim, 25, 7-8 (2000).

Engelsman M, Damen EMF, Koken PW, van 't Veld AA, van Ingen $K M$ and Mijnheer BJ. Impact of simple tissue inhomogeneity correction algorithms on conformal radiotherapy of lung tumours. Radiother Oncol, 60, 299-309 (2001).

Essers $\mathrm{M}$ and Mijnheer BJ. In vivo dosimetry during external photon beam radiotherapy. Int J Radiol Biol Phys, 43, 245-259 (1999).

Essers $\mathrm{M}$ and Mijnheer BJ. In vivo dosimetry during external photon beam radiotherapy. Int J Radiat Oncol Biol Phys, 43, 245-259 (1999).

Essers M, Lanson JH, Leunens G, Schnabel T and Mijnheer BJ. The accuracy of CTbased inhomogeneity corrections and in vivo dosimetry for the treatment of lung cancer. Radiotherapy and Oncology, 37, 199-208 (1995).

Evans RD. The Atomic Nucleus. New York: McGraw Hill Book Company, Inc. (1955).

Faddegon B, Schreiber E and Ding X. Monte Carlo simulation of large electron fields. Phys Med Biol, 50, 1-13 (2005).

Fippel M, Haryanto F, Dohm O, Nusslin F and Kriesen S. A virtual photon energy fluence model for Monte Carlo dose calculation. Med Phys, 30, 301-11 (2003).

Fippel M. Fast Monte Carlo dose calculation for photon beams based on the VMC electron algorithm. Med Phys, 26, 1466-75 (1999).

Fix MK, Keall PJ, Dawson K and Siebers JV. Monte Carlo source model for photon beam radiotherapy: photon source characteristics. Med Phys, 31, 3106-21 (2004). 
Gagné IM and Zavgorodni S. Evaluation of the analytical anisotropic algorithm in an extreme water-lung interface phantom using Monte Carlo dose calculations. J App Clin Med Phys, 8, 33-46 (2007).

Goitein M. Calculation of the uncertainty in the dose delivered during radiation therapy. Med Phys, 12, 608-612 (1985).

Graham MV, Purdy JA, Emami B, et al. Clinical dose-volume histogram analysis for pneumonitis after 3D treatment for non-small cell lung cancer. Int J Radiat Oncol Biol Phys, 45, 323-329 (1999).

Groh BA, Siewerdsen JH, Drake DG, Wong JW and Jaffrey DA. A performance comparison of flat-panel imager-based MV and kV cone-beam CT. Med Phys, 29, 967-975 (2002).

Gustafsson A, Lind BK and Brahme A. A generalized pencil beam algorithm for optimization of radiation therapy. Med Phys, 21, 343-356 (1994).

Haedinger U, Kreiger T, Flentje M and Wulf J. Influence of calculation model on dose distribution in stereotactic radiotherapy for pulmonary targets. Int J Radiat Onc Biol Phys, 61, 239-249 (2005).

Hazuka MB, Ibbott GS and Kinzie JJ. Hip prostheses during pelvic irradiation: effects and corrections. Int J Radiat Oncol Biol Phys, 14, 1311-1317 (1988).

Heath E. Evaluation of the PEREGRINE Monte Carlo dose calculation code for 6 MV photon beams, MSc Thesis. Montreal: McGill University (2003).

Heath E and Seuntjens J. Development and validation of a BEAMnrc component module for accurate Monte Carlo modeling of the Varian dynamic Millenium multileaf collimator. Phys Med Biol, 48, 4045-63 (2003).

Hernando ML, Marks LB, Bentel GC, et al. Radiation-induced pulmonary toxicity: a dose-volume histogram analysis in 201 patients with lung cancer. Int J Radiat Oncol Biol Phys, 51, 650-659 (2001).

Higgins DM, Whitehurst $\mathrm{P}$ and Morgan AM. The effect of carbon fiber couch inserts on surface dose with beam size variation. Med Dosim, 26, 251-254 (2001).

Hoban PW, Murray DC and Round WH. Photon beam convolution using polyenergetic energy deposition kernels. Phys Med Biol, 39, 669-685 (1994).

Hudson FR, Crawley MT and Samarasekera M. Radiotherapy treatment planning for patients fitted with prostheses. Br J Radiol, 57, 603-608 (1984). 
International Atomic Energy Agency. Review of Radiation Oncology Physics: A Handbook for Teachers and Students, ed. Podgorsak EB. Vienna, Austria: IAEA (2003).

International Commission on Radiation Units and Measurements. ICRU Report 37: Stopping powers for electrons and positrons. ICRU, Washington D.C. (1984).

International Commission on Radiation Units and Measurements. ICRU Report 50: Prescribing, Recording, and Reporting Photon Beam Therapy. Bethesda, Maryland: ICRU, 1-72 (1993).

International Commission on Radiation Units and Measurements. ICRU Report 62: Prescribing, Recording, and Reporting Photon Beam Therapy (Supplement to ICRU Report 50). Bethesda, Maryland: ICRU (1999).

Jiang SB, Boyer AL and Ma CM. Modeling the extrafocal radiation and monitor chamber backscatter for photon beam dose calculation. Med Phys, 28, 55-66 (2001).

Jeraj R, Mackie TR, Balog J, Olivera G, Peason D, Kapatoes J, Ruchala K and Reckwerdt P. Radiation characteristics of helical tomotherapy. Med Phys, 31, 396404 (2004).

Johns HE and Cunningham JR. The Physics of Radiology, $4^{\text {th }}$ Ed. Springfield, Illinois, USA: Charles C Thomas (1983).

Kachelriess M, Watzke $\mathrm{O}$ and Kalender, WA. Generalized multidimensional adaptive filtering for conventional and spiral single-slice, multi-slice, and cone-beam CT. Med Phys, 28, 475-490 (2001).

Kawrakow I and Rogers DWO. The EGSnrc Code System: Monte Carlo simulation of electron and photon transport, PIRS-701. National Research Council of Canada, Ottawa, pp13-16 (2006).

Kawrakow I. VMC++, electron and photon Monte Carlo calculations optimized for radiation treatment planning, Advanced Monte Carlo for Radiation Physics, Particle Transport Simulation and Applications: Proceedings of the Monte Carlo Meeting, ed Kling A, Barao F, Nakagawa M et al. New York: Springer, 229-236 (2000).

Keall PJ and Hoban PW. Super-Monte Carlo: a 3-D electron beam dose calculation algorithm. Med Phys, 23, 2023-34 (1996).

Klein EE, Morrison A, Purdy JA, Graham MV and Matthews J. A volumetric study of measurements and calculations of lung density corrections for 6 and $18 \mathrm{MV}$ photons. Int J Radiat Oncol Biol Phys, 37, 1163-1170 (1997). 
Knöös T, Ahnesjö A, Nilsson P and Weber L. Limitations of a pencil beam approach to photon dose calculations in lung tissue. Phys Med Biol, 40, 1411-1420 (1995).

Knöös T, Wieslander E, Cozzi L, Brink C, Fogliata A, Albers D, Nyström and Lassen $\mathrm{S}$. Comparison of dose calculation algorithms for treatment planning in external photon beam therapy for clinical situations. Phys Med Biol, 51, 5785-5807 (2006).

Kron T, Grigorov G, Yu E, Yartsev S, Chen JZ, Wong E, Rodrigues G, Trenka K, Coad T, Bauman G and Van Dyk J. Planning evaluation of radiotherapy for complex lung cancer cases using helical tomotherapy. Phys Med Biol, 49, 3675-3690 (2004).

Kron T. Thermoluminescence dosimetry and its applications in medicine - part 2: history and applications. Australas Phys Eng Sci Med, 18, 1-25 (1995).

Langen KM, Meeks SL, Poole DO, Wagner TH, Willoughby TR, Kupelian PA, Ruchala KJ, Haimerl J and Olivera GH. The use of megavoltage CT (MVCT) images for dose recomputations. Phys Med Biol, 50, 4259-4276 (2005).

Laub W, Bakai A and Nüsslin F. Intensity modulated irradiation of a thorax phantom: comparisons between measurements, Monte Carlo calculations and pencil beam calculations. Phys Med Biol, 46, 1695-1706 (2001).

Laub WU and Nüsslin F. Monte Carlo dose calculations in the treatment of a pelvis with implant and comparison with pencil-beam calculations. Med Dosim, 28, 229233 (2003).

Mackie TR, Bielajew AF, Rogers DWO and Battista JJ. Generation of photon energy deposition kernels using the EGS Monte Carlo code. Phys Med Biol, 33, 1-20 (1988).

Mackie TR, El-Khatib E, Battista J, Scrimger J, Van Dyk J and Cunningham JR. Lung dose corrections for 6- and 15-MV x rays. Med Phys, 12, 327-332 (1985).

Mackie TR, Scrimger JW and Battista JJ. A convolution method of calculating dose for 15-MV x-rays. Med Phys, 12, 188-196 (1985).

Mah K and Van Dyk J. On the impact of tissue inhomogeneity corrections in clinical thoracic radiation therapy. Int J Radiat Oncol Biol Phys, 21, 1257-1267 (1991).

McCall RC, McIntyre RD and Turnbull WG. Improvement of linear accelerator depth-dose curves. Med Phys, 5, 518-24 (1978).

McCollough $\mathrm{CH}$ and Zink FE. CT numbers and electron density, Imaging in radiotherapy, ed Hazle J and Boyer A, Madison, WI: Medical Physics Publishing, 135-161 (1998). 
McCormack S, Diffey J and Morgan A. The effect of gantry angle on megavoltage photon beam attenuation by a carbon fiber couch insert. Med Phys, 32, 483-487 (2005).

Meara SJP and Langmack KA. An investigation into the use of carbon fiber for megavoltage radiotherapy applications. Phys Med Biol, 43, 1359-1366 (1998).

Meeks SL, Harmon Jr JF, Langen KM, Willoughby TR, Wagner TH and Kupelian PA. Performance characterization of megavoltage computed tomography imaging on a helical tomotherapy unit. Med Phys, 32, 2673-2681 (2005).

Metcalfe P, Wong $\mathrm{T}$ and Hoban P. Radiotherapy $\mathrm{x}$-ray beam inhomogeneity corrections: the problem of lateral electronic disequilibrium in lung. Australas Phys Eng Sci Med, 16, 155-67 (1993).

Meyer J, Mills JA, Haas OC, Burnham KJ and Parvin EA. Accommodation of couch constraints for coplanar intensity modulated radiation therapy. Radiother Oncol, 61, 23-32 (2001).

Miften M, Wiesmeyer M, Monthofer S and Krippner K. Implementation of FFT convolution and multigrid superposition models in the FOCUS RTP system. Phys Med Biol, 45, 817-833 (2000).

Mijnheer BJ, Battermann JJ and Wambersie A. "What degree of accuracy is required and can be achieved in photon and neutron therapy?" Radiotherapy and Oncology, 8 , 237-252 (1987).

Mohan R, Chui C and Lidofsky L. Differential pencil beam dose computation model for photons. Med Phys, 13, 64-73 (1986).

Morin O, Chen J, Aubin M, Gillis A, Aubry JF, Bose S, Chen H, Descovich M, Xia P and Pouliot J. Dose calculation using megavoltage cone-beam CT. Int J Radiat Oncol Biol Phys, 15, 1201-1210 (2007).

Morin R and Raeside DE. Removal of streaking artifact in computed tomography. J Med Syst, 6, 387-297 (1982).

Muthuswamy MS and Lam KL. A method of beam-couch intersection detection. Med Phys, 26, 229-235 (1999).

Nilsson $\mathrm{M}$ and Knöös T. Application of the Fano theorem in inhomogeneous media using a convolution algorithm. Phys Med Biol, 37, 69-83 (1992). 
Orton CG, Chungbin S, Klein EE, Gillin MT, Schulteiss TE and Sause WT. Study of lung density corrections in a clinical trial (RTOG 88-08). Int J Radiat Oncol Biol Phys, 41, 787-794 (1998).

Ostapiak OZ, Zhu Y and Van Dyk J. Refinements of the finite-size pencil beam model of three-dimensional photon dose calculation. Med Phys, 24, 743-750 (1997).

Papanikolaou $\mathrm{N}$ and Klein EE. Point/Counterpoint: Heterogeneity corrections should be used in treatment planning for lung cancer. Med Phys, 27, 1702-1704 (2000).

Pena J, Franco L, Gomez F, Iglesias A, Lobato R, Mosquera J, Pazos A, Pardo J, Pombar M, Rodriguez A and Sendon J. Commissioning of a medical accelerator photon beam Monte Carlo simulation using wide field profiles. Phys Med Biol, 49, 4929-42 (2004).

Perez CA and Brady LW. Principles and practice of radiation oncology, $3^{\text {rd }}$ Edition. Lippencott, Williams and Wilkins, 1-10 (1997).

Petoukhova AL, Terhaard $\mathrm{CHJ}$ and Welleweerd H. Does $4 \mathrm{MV}$ perform better compared to $6 \mathrm{MV}$ in the presence of air cavities in the head and neck region? Radiotherapy and Oncology, 79, 203-207 (2006).

Prasad SG, Parthasaradhi K and Bloomer WD. Effective atomic numbers of composite materials for total and partial interaction processes for photons, electrons, and protons. Med Phys, 24, 883-885 (1997).

Ramsey CR, Cordrey IL, Spencer KM and Oliver AL. Dosimetric verification of two commercially available three-dimensional treatment planning systems using the TG 23 test package. Med Phys, 26, 1188-1195 (1999).

Reft C, Alecu R, Das IJ, Gerbi BJ, Keall P, Lief E, Mijnheer BJ, Papanikolaou N, Sibata $\mathrm{C}$ and Van Dyk J. Dosimetric considerations for patients with HIP prostheses undergoing pelvic irradiation. Report of the AAPM Radiation Therapy Committee Task Group 63. Med Phys, 30, 1162-1182 (2003).

Roberts R. How accurate is a CT-based dose calculation on a pencil beam TPS for a patient with a metallic prosthesis. Phys Med Biol, 46, N227-N234 (2001).

Robertson DD, Yuan J, Wang G and Vannier MW. Total hip prosthesis metal-artifact suppression using iterative deblurring reconstruction. J Comp Assis Tomogr, 21, 293-298 (1997).

Rogers DWO and Bielajew AF. Wall attenuation and scatter corrections for ion chambers: measurements versus calculations. Phys Med Biol, 35, 1065-78 (1990). 
Rogers DWO, Bielajew AF and Nahum AE. Ion chamber response and Awall correction factors in a ${ }^{60} \mathrm{Co}$ beam by Monte Carlo simulation. Phys Med Biol, 30, 429-43 (1985).

Rogers DWO, Faddegon BA, Ding GX, Ma C-M, We J and Mackie TR. BEAM: A Monte Carlo code to simulate radiotherapy treatment units. Med Phys, 22, 503-24 (1995).

Rogers DWO, Ma C-M, Walters B, Ding GX, Sheikh-Bagheri D and Zhang G. BEAMnrc Users Manual, PIRS-0509. 66-67 (2002).

Ruchala KJ, Olivera GH, Schloesser EA, Hinderer R, Mackie TR. Calibration of a tomotherapeutic MVCT system. Phys Med Biol. 2000, N27-N36 (2000).

Ruchala KJ, Olivera GH, Kapatoes JM, Schloesser EA, Reckwerdt PJ and Mackie TR. Megavoltage CT image reconstruction during tomotherapy treatments. Phys Med Biol, 45, 2545-2562 (2000).

Ruchala KJ, Olivera GH, Schloesser EA and Mackie TR. Megavoltage CT on a tomotherapy system. Phys Med Biol, 44, $2597-2621$ (1999).

Sanchez-Nieto B and Nahum AE. The delta-TCP concept: A clinically useful measure of tumor control probability. Int J Rad Oncol Biol Phys, 44, 369-380 (1999).

Sempau J, Wilderman SJ and Bielajew AF. DPM, a fast, accurate Monte Carlo code optimized for photon and electron radiotherapy treatment planning dose calculations. Phys Med Biol, 45, 2263-91 (2000).

Sheikh-Bagheri D and Rogers DWO. Sensitivity of megavoltage photon beam Monte Carlo simulations to electron beam and other parameters. Med Phys, 29, 379-390 (2002).

Sheikh-Bagheri D, Rogers DWO, Ross CK and Seuntjens JP. Comparison of measured and Monte Carlo calculated dose distributions from the NRC linac. Med Phys, 27, 2256-66 (2000).

Shin $\mathrm{H}$. Influence of hip prostheses on high energy photon dose distribution. Int $\mathbf{J}$ Radiat Oncol Biol Phys, 18, 455-461 (1990).

Simpson RG, Chen CT, Grubbs EA and Swindell W. A 4-MV CT scanner for radiation therapy: the prototype system. Med Phys, 9, 574-579 (1982).

Verhaegen F, Symonds-Taylor R, Liu HH and Nahum AE. Backscatter towards the monitor ion chamber in high energy photon and electron beams: charge integration versus Monte Carlo simulation. Phys Med Biol, 45, 3159-3170 (2000). 
Vieira SC, Kaatee RSJP, Dirkx MLP, Heijmen BJM. Two-dimensional measurement of photon beam attenuation by the treatment couch and immobilization devices using an electronic portal imaging device. Med Phys, 30, 2981-2987 (2003).

Walters BRB, Kawrakow I and Rogers DWO. DOSXYZnrc User's Manual, NRC Report PIRS 794 (rev B) (2004).

Wang L, Zhu Y and Jette D. Analytic modeling of the primary X-ray dose deposition kernels. Med Phys, 22, 977 (1995).

Webb S. The absorbed dose in the vicinity of an interface between two media irradiated by a ${ }^{60}$ Co source. Br J Radiol, 52, 962-967 (1979).

Weber L and Nilsson P. Verification of dose calculations with a clinical treatment planning system based on a point kernel dose engine. J App Clin Med Phys, 3, 73-87 (2002).

White PJ, Zwicker RD and Huang DT. Comparison of dose homogeneity effects due to electron equilibrium loss in lung for $6 \mathrm{MV}$ and $18 \mathrm{MV}$ photons. Int J Radiat Oncol Biol Phys, 34, 1141-1146 (1996).

Woo MK and Cunningham JR. The validity of the density scaling method in primary electron transport for photon and electron beams. Med Phys, 17, 187-194 (1990).

Yorke ED. The geometry of avoiding beam intersections and blocking tray collisions. Med Phys, 16, 288-291 (1989).

Yu C and Luxton G. TLD dose measurement: A simplified accurate technique for the dose range from $0.5 \mathrm{cGy}$ to $1000 \mathrm{cGy}$. Med Phys, 26, 1010-1016 (1999). 